\title{
Water-Use Data by Category, County, and Water Management District in Florida, 1950-90
}

By Richard L. Marella

U.S. GEOLOGICAL SURVEY

Open-File Report 94-521

Prepared in cooperation with the

Florida Department of Environmental Protection

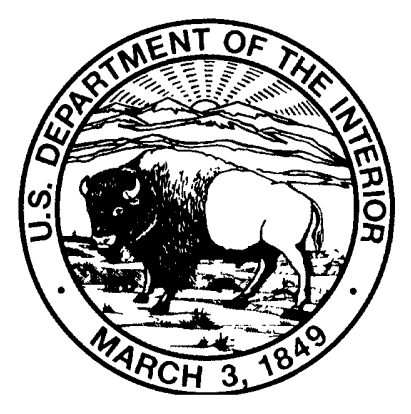




\title{
U.S. DEPARTMENT OF THE INTERIOR BRUCE BABBITT, Secretary
}

\author{
U.S. GEOLOGICAL SURVEY \\ Gordon P. Eaton, Director
}

\begin{abstract}
Any use of trade, product, or firm names in this publication is for descriptive purposes only and does not imply endorsement by the U.S. Geological Survey.
\end{abstract}

For additional information write to:

Copies of this report can be purchased from:

District Chief

U.S. Geological Survey

U.S. Geological Survey

Suite 3015

Earth Science Information Center

227 North Bronough Street

Open-File Reports Section

Tallahassee, Florida 32301

P.O. Box 25286, MS 517

Denver, CO 80225-0425 


\section{CONTENTS}

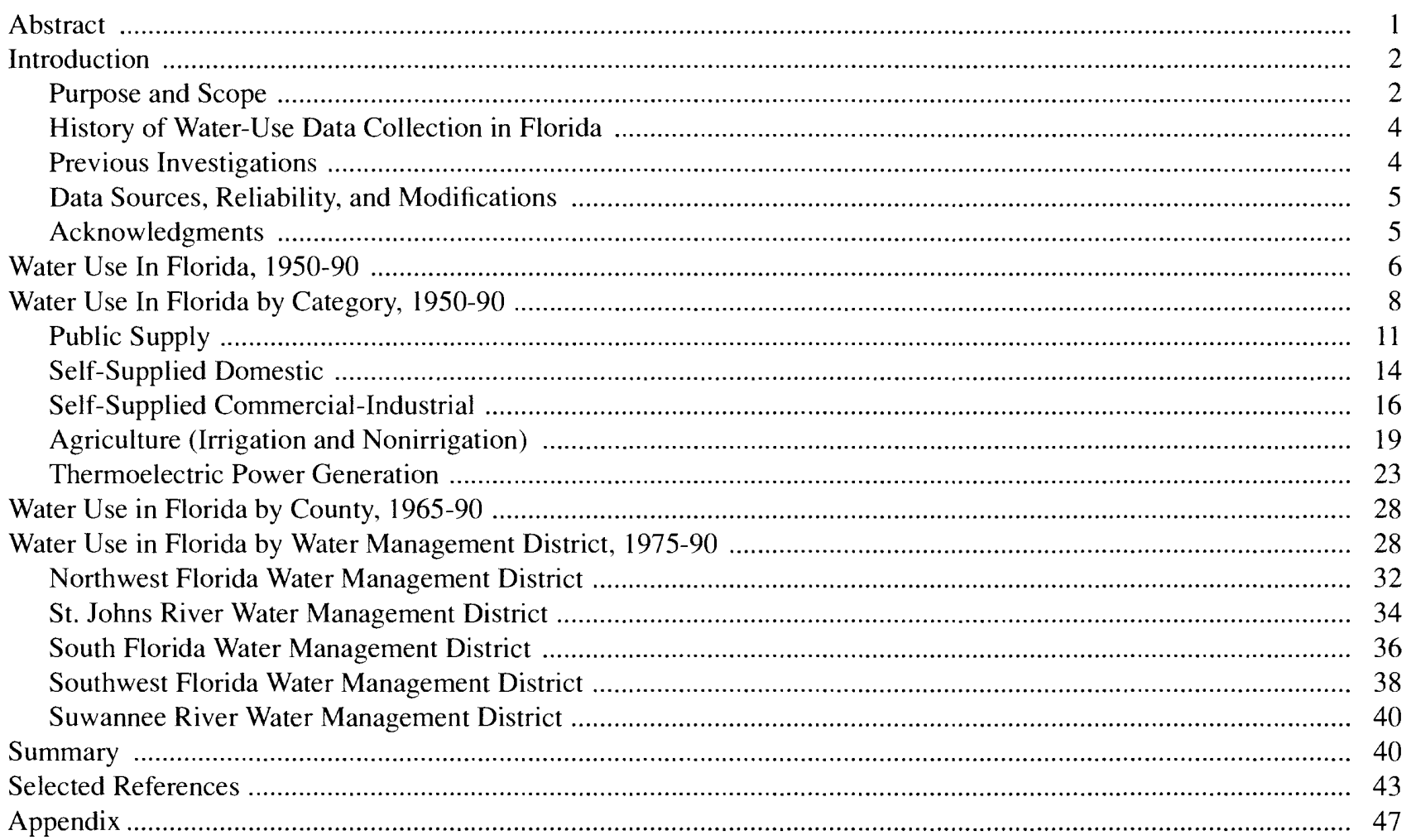

\section{FIGURES}

1. Graph showing population of Florida, 1950-2020

2. Map showing counties and selected place names in Florida ....................................................................... 3

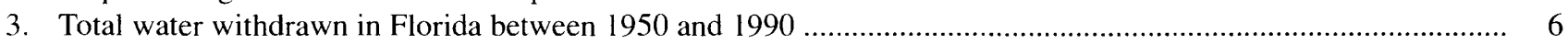

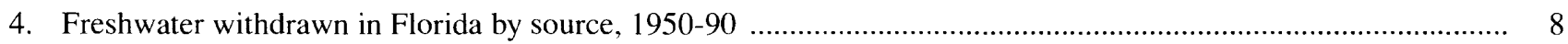

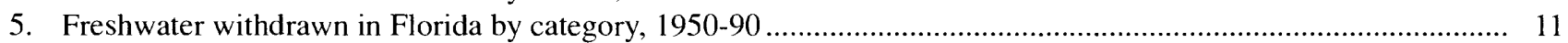

6. Public-supply freshwater withdrawn in Florida by source, 1950-90 …........................................................ 13

7. Self-supplied domestic ground water withdrawn in Florida, 1950-90 …................................................. 16

8. Self-supplied commercial-industrial freshwater withdrawn in Florida by source, 1950-90 ............................. 18

9. Agriculture (irrigation and nonirrigation) freshwater withdrawn in Florida by source, 1950-90 ....................... 21

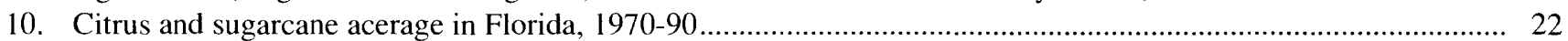

11. Thermoelectric power generation water withdrawn in Florida by source, 1950-90 _............................................ 25

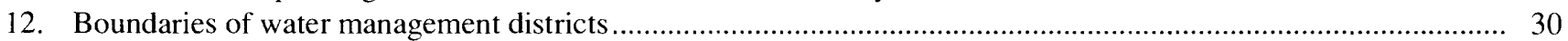

13. Freshwater withdrawn by water management district, $1975-90$...................................................................... 31

14. Freshwater withdrawin in the Northwest Florida Water Management District by source, 1975, 1980, 1985 and 1990

15. Freshwater withdrawn in the St. Johns River Water Management District by source, 1975, 1980, 1985 and 1990.

16. Freshwater withdrawn in the South Florida Water Management District by source, $1975,1980,1985$ and 1990. 
17. Freshwater withdrawn in the Southwest Florida Water Management District by source, $1975,1980,1985$ and 1990

18. Freshwater withdrawn in the Suwannee River Water Management District by source,

$1975,1980,1985$ and 1990

\section{TABLES}

1. Total water withdrawals in Florida by source, 1950-90

2. Freshwater withdrawn and percent change in Florida by county, 1965-90

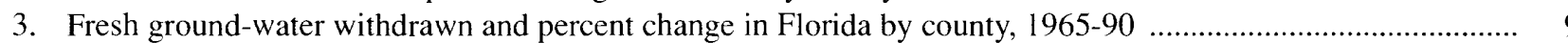

4. Fresh surface-water withdrawn and percent change in Florida by county, 1965-90 ..................................... 10

5. Freshwater withdrawals in Florida by category, 1965-90 ...................................................................... 12

6. Public-supply population served and freshwater withdrawals in Florida by source, 1950-90 _......................... 13

7. Public-supply freshwater use and percent change in Florida by county, 1965-90 ......................................... 15

8. Self-supplied domestic population and freshwater withdrawals in Florida by source, 1950-90 ....................... 16

9. Self-supplied domestic freshwater use and percent change in Florida by county, 1965-90 .......................... 17

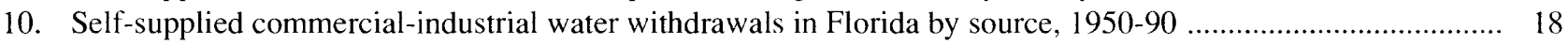

11. Self-supplied commercial-industrial freshwater use and percent change in Florida by county, 1965-90 ............ 20

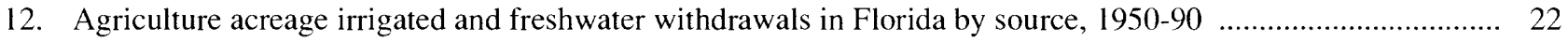

13. Agriculture freshwater use and percent change in Florida by county, 1965-90 ........................................... 24

14. Thermoelectric power generation water withdrawals in Florida by source, $1950-90$.................................... 26

15. Thermoelectric power generation freshwater use and percent change in Florida by county, 1965-90 ................ 27

16. Freshwater withdrawal data availability in Florida by county, 1970-90 ..................................................... 29

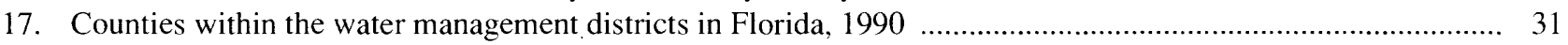

18. Freshwater withdrawals by category in the Northwest Florida Water Management District, 1975-90 _............. 33

19. Freshwater withdrawals by category in the St. Johns River Water Management District, 1975-90 ................... 35

20. Freshwater withdrawals by category in the South Florida Water Management District, 1975-90 ..................... 37

21. Freshwater withdrawals by category in the Southwest Florida Water Management District, 1975-90 ............... 39

22. Freshwater withdrawals by category in the Suwannee River Water Management District, 1975-90 ................. 41

\section{APPENDIX TABLES}

1-60. Freshwater withdrawals by category, $1965-90$, for all counties:

1. Alachua County ............................................ 48

2. Baker County ................................................ 49

3. Bay County ................................................ 50

4. Bradford County ............................................ 51

5. Brevard County .............................................. 52

6. Broward County …......................................... 53

7. Calhoun County ………………………........ 54

8. Charlotte County ............................................ 55

9. Citrus County ……………………………….... 56

10. Clay County …............................................ 57

11. Collier County ............................................... 58

12. Columbia County .......................................... 59

13. Dade County ................................................... 60

14. De Soto County, 1965-9 ................................. 61

15. Dixie County ……………………………..... 62

16. Duval County ................................................ 63

17. Escambia County ............................................. 64

18. Flagler County ............................................. 65
19. Franklin County .............................................. 66

20. Gadsden County ............................................ 67

21. Gilchrist County ............................................. 68

22. Glades County ................................................. 69

23. Gulf County .............................................. 70

24. Hamilton County ........................................... 71

25. Hardee County ............................................... 72

26. Hendry County ............................................... 73

27. Hernando County .......................................... 74

28. Highlands County ………………………... 75

29. Hillsborough County ...................................... 76

30. Holmes County ............................................. 77

31. Indian River County ....................................... 78

32. Jackson County ............................................ 79

33. Jefferson County ............................................ 80

34. Lafayette County …........................................ 81

35. Lake County ................................................ 82

36. Lee County ............................................... 83 
37. Leon County

38. Levy County

39. Liberty County

40. Madison County

41. Manatee County

42. Marion County

43. Martin County

44. Monroe County

45. Nassau County

46. Okaloosa County

47. Okeechobee County

48. Orange County

49. Osceola County

50. Palm Beach County

51. Pasco County

52. Pinellas County
53. Polk County 100

54. Putnam County 101

55. St. Johns County 102

56. St. Lucie County 103

57. Santa Rosa County 104

58. Sarasota County

59. Seminole County

60. Sumter County

61. Suwannee County

62. Taylor County

63. Union County

64. Volusia County

65. Wakulla County

66. Walton County

67. Washington County

\section{CONVERSION FACTORS, ABBREVIATIONS AND ACRONYMS}

\begin{tabular}{rcl}
\hline Multiply & By & To obtain \\
\hline acre & 4,047 & square meter \\
acre & 0.00156 & square mile \\
square mile & 2.59 & square kilometer \\
gallons per day $(\mathrm{gal} / \mathrm{d})$ & 3.785 & liters per day \\
gigawatthour $(\mathrm{GWh})$ & 1,000 & megawatthour \\
$\mathrm{GWh}$ & $1,000,000$ & kilowatthour \\
million gallons per day $(\mathrm{Mgal} / \mathrm{d})$ & 0.003785 & million cubic meters per day \\
\hline
\end{tabular}

ASCS = Agricultural Stabilization and Conservation Services

FBOG $=$ Florida Bureau of Geology

FDEP $=$ Florida Department of Environmental Protection

FDER = Florida Department of Environmental Regulation

NWFWMD = Northwest Florida Water Management District

SCS = Soil Conservation Service

SJRWMD = St. Johns River Water Management District

SFWMD = South Florida Water Management District

SWFWMD = Southwest Florida Water Management District

SRWMD = Suwannee River Water Management District

USGS = U.S. Geological Survey 


\section{GLOSSARY}

Agriculture water use.--Includes water used for agricultural irrigation and nonirrigation purposes. Irrigation water use includes the artificial application of water on lands to assist in the growing of crops, plants, and pasture, or to maintain vegetative growth in recreational lands, parks, and golf courses. Nonirrigation water use includes water used for livestock, fish farming, and other farm needs. Livestock water use includes water used for stock watering, feedlots, and dairy operations.

Commercial water use.--Water for motels, hotels, restaurants, office buildings, commercial facilities and civilian and military institutions. The water may be obtained from a public supply or may be self-supplied.

Consumptive use.--That part of water withdrawn that is evaporated, transpired, incorporated into products or crops, consumed by humans or livestock, or otherwise removed from the immediate water environment. Sometimes called water consumed or water depleted. Additionally, any water withdrawn and transferred out of a county or hydrologic basin for use is considered 100 percent consumptively used in that county or hydrologic basin.

Cooling pond.--Usually a man-made water body used by power plants or large industrial plants that enables the facility to recirculate once-through cooling water. The water levels in the pond are usually maintained by rainfall or augmented by pumping (withdrawal of) water from another source (fresh, saline, or reclaimed).

Cooling tower---A large tower or stack that is used for heat exchange of once-through cooling water generated by steam condensers. Hot water from the plant is sprayed into the top of the tower and exchanges heat with the passing air as it falls. The water is then collected at the bottom of the tower and used again. A small amount of water is lost (consumed) through evaporation in this process. See cooling water or once-through cooling.

Cooling water.--Water used for cooling purposes by electric generators, steam condensers, large machinery or products at power or industrial plants. Water used for cooling purposes can be either fresh, saline, or reclaimed and may be used only once or recirculated multiple times. See cooling pond or once-through cooling.

Desalination.--Refers to the removal of salts from water. Desalination is primarily used for public-supply water to ensure that it meets Florida Department of Environmental Protection secondary drinking standards. The three primary types of desalination used in Florida are: (1) distillation, (2) electrodialysis processes, and (3) reverse osmosis processes (Buros, 1989, South Florida Water Management District, 1990). The reverse osmosis processes are the most commonly used in Florida followed by electrodialysis (Dykes and Conlon, 1989). In addition to these three desalination processes, many public suppliers also dilute or blend brackish or saline water with fresher water to produce potable water. Also see Reverse osmosis.

Dewatering.--The deliberate attempt to lower the ground-water level in or below land surface for selected purposes such as agricultural, construction, mining or other activities. For mining operation, dewatering usually is accomplished by pumping the water out of the ground and discharging to a surface-water body. However, some dewatering involves gravity feeding water from the surficial aquifer into a deeper aquifer (usually the Floridan aquifer system) through recharge wells (Campbell, 1986, p. 36). In Florida, this discharge usually requires a permit from the Florida Department of Environmental Protection.

Domestic wastewater facility.--Refers to those facilities that receive or dispose of wastewater derived principally from residential dwellings, business or commercial buildings, institutions, and the like (Marella, 1994). Can also include some wastewater derived from industrial facilities. May also be referred to as a municipal wastewater facility. 
Domestic water use.--Water for normal household purposes, such as drinking, food preparation, bathing, washing clothes and dishes, flushing toilets, and watering lawns and gardens. Also called residential water use. The water can be obtained from a public supply or be self-supplied.

Effluent.--Refers to the water that flows out of a wastewater treatment facility or other works used for the purpose of treating, stabilizing, or holding waste.

Flood irrigation.-- 1rrigation systems that control the water table with lateral supply ditches. These include open field ditch systems (furrows), semi-closed conveyance systems, subsurface conduit systems, crown flood systems, and continuous flood systems. Also includes seepage or subsurface irrigation systems. The efficiencies of these flood irrigation systems range from 20 to 80 percent (Smajstrla and others, 1988), however, an average of 60 percent is commonly used for estimating water requirements.

Freshwater.--Water that contains less than 1,000 milligrams per liter $(\mathrm{mg} / \mathrm{L})$ of dissolved solids; generally, more than $500 \mathrm{mg} / \mathrm{L}$ is considered undesirable for drinking and many industrial uses. Generally, freshwater is considered potable.

Gigawatthour (GWh).--A measure of electricity, one billion watthours.

Ground water.--Specifically, that part of the subsurface water that is in the saturated zone (a zone in which all voids are filled with water).

Hydroelectric power water use.--The use of water in the generation of electricity at plants where the turbine generators are driven by falling water. This is considered an instream use of water.

Industrial wastewater facility.--Refers to those facilities that produce, treat or dispose of wastewater not otherwise defined as a domestic wastewater; includes the runoff and leachate from areas that receive pollutants associated with industrial or commercial storage, handling, or processing (Marella, 1994).

Industrial water use.--Water used for industrial purposes such as fabricating, processing, washing, and cooling, and includes such industries as steel, chemical and allied products, paper and allied products, mining, and petroleum refining. The water can be obtained from a public supply or be self-supplied.

Instream use.--Water use taking place within a stream channel for such purposes as hydroelectric power generation, navigation, water-quality improvement, fish and wildlife propagation, and recreation. Sometimes called nonwithdrawal use or in-channel use.

Low pressure/low volume irrigation.--Irrigation systems that apply water directly to, or very near, the soil surface, either above the ground or into he air, in discrete drops, continuous drops, small streams, mist, or sprays. These include drip systems, spray systems, jet systems, and bubbler systems. Also referred to as micro- or trickleirrigation. The efficiencies of these low pressure irrigation systems range from 75 to 95 percent (Smajstrla and others, 1988), however, an average of 80 percent is commonly used for estimating water requirements.

Mining water use.--Water used for the extraction of minerals and liquids. Mining also includes water used for milling (such as crushing, screening, washing, and flotation), environmental purposes (such as dust control and wetland restoration or maintenance), material conveyance, dewatering, and domestic uses on the premises. Generally, most of the water used at a mining operation is self-supplied.

Navigational water use.--Water utilized as a means of commercial (and sometimes recreational) transportation. Includes water used to lift a vessel in a lock, or maintain a navigable channel level. Navigational water use is considered a non-consumptive instream use of water and is generally not measured or taken into account. 
Net water-use.--Refers to water withdrawals plus or minus water transfers. In most counties, the net water-use and water withdrawals are equal. However, in counties involved in water transfers (imports and exports), the net water-use represents the actual amount of water used regardless of the amount of water withdrawn. In Florida, water transfers are mostly found in the public supply category. Also see water transfers.

Once-through cooling water.--Water (fresh or saline) that is withdrawn from a river, stream or other water body (man-made or natural), or a well, that is passed through a steam condenser one time, and then returned to the river or stream or other water body some distance from the intake (Hughes, 1975, p. 14). Once-through cooling water is used to exchange the heat from the steam condensers to the cooler water. This method of cooling is commonly used in power production throughout Florida, and usually results in very little, if any, consumption.

Offstream use.--Water withdrawn or diverted from a ground- or surface-water source for public-water supply, industry, irrigation, livestock, thermoelectric power generation, and other uses. Sometimes called off-channel use or withdrawal use.

Other water use.--Water used in Florida for such purposes as heating, cooling, irrigation (public-supplied only), lake augmentation, and other nonspecific uses. The water can be obtained from a public supply or be self supplied.

Per capita use.--The average amount of water used per person during a standard time period, generally per day.

Potable water.-- Refers to water that meets the quality standards set by the Florida Department of Environmental Protection. Potable water is considered safe for human consumption and is often referred to as drinking water. In Florida, chloride and dissolved-solids concentrations in potable water must be less than or equal to $250 \mathrm{mg} / \mathrm{L}$ and $500 \mathrm{mg} / \mathrm{L}$, respectively. Freshwater that exceeds these chloride and dissolved solids limits is often referred to as slightly saline, brackish, or nonpotable water and is either diluted with fresher water or treated through a desalination process to meet potable-water standards for public supply.

Public supply.--Water withdrawn by public or private water suppliers and delivered to users who do not supply their own water. Water suppliers provide water for a variety of uses, such as domestic, commercial, industrial, thermoelectric power (domestic and cooling purposes), and public-water use. According to the Florida Department of Environmental Protection, any water system that serves more than 25 people or has 15 year-round service connections is considered a public supplier (Florida Department of Environmental Regulation, 1990, p. 6).

Public-water use.--Water supplied from a public-water supply and used for such purposes as firefighting, street washing, and municipal parks and swimming pools. Publicwater use also includes system water losses (water lost to leakage) and unusable water discharged from desalination or lime-softening facilities. Also referred to as utility-water use.

Reclaimed water.--Refers to water that has received at least secondary treatment and is reused after leaving a wastewater treatment facility.

Recycled water.--Water that is used more than one time before it passes back into the natural hydrological system or is discharged into a wastewater system. Also referred to as recirculated water.

Resident population.--The number of persons who live in a State who consider the State their permanent place of residence. College students, military personnel, and inmates of penal institutions are counted as permanent residents. According to this definition, tourist and seasonal or part-time residents are considered nonresident population.

Residential water use.--See domestic water use. 
Reuse system.--Means the deliberate application of reclaimed water for a beneficial or other useful purpose. Reuse may encompass landscape irrigation (such as golf courses, cemeteries, highway medians, parks, playgrounds, school yards, nurseries, and residential properties), agricultural irrigation (such as food and fruit crops, wholesale nurseries, sod farms and pasture grass), aesthetic uses, ground-water recharge, environmental enhancement of surface water and wetland restoration, fire protection, and other useful purposes.

Reverse osmosis.--Refers to the process of removing salts from water using a membrane. With reverse osmosis, the product water passes through a fine membrane that the salts are unable to pass through, and the salt waste (brine) is removed and disposed. This differs from electrodialysis where the salts are extracted from the feedwater by using a membrane with an electrical current to separate the ions. The positive ions flow through one membrane, while the negative ions flow through a different membrane, leaving freshwater as the end product. In this report, reverse osmosis includes any water treated through both reverse osmosis and electrodialysis and any water diluted or blended with fresher water that was used to obtain potable water. Also see desalination.

Saline water---Water that contains more than $1,000 \mathrm{mg} / \mathrm{L}$ of dissolved solids.

Self-supplied water---Water withdrawn from a ground- or surface-water source by a user and not obtained from a public supply.

Sprinkler irrigation.--A pressurized irrigation system where water is distributed through pipes to the field and applied through a variety of sprinkler heads or nozzles. Pressure is used to spread water droplets above the crop canopy to simulate a rainfall (Izuno and Haman, 1987). These systems include portable and traveling guns, solid or permanent fixtures (overhead or pop ups), center pivots, and periodic moving systems. Also referred to as overhead irrigation. The efficiencies of these sprinkler irrigation systems range from 15 to 85 percent (Smajstrla and others, 1988), however, an average of 70 percent is commonly used for estimating water requirements.

Tail-water runoff.--Refers to unused irrigation water or rain water that is collected at the base or end of an irrigated system or field in a ditch or impoundment. This water may be reused again for irrigation purposes, left to evaporate, percolate into the ground, treated, and (or) discharged to surface water bodies.

Thermoelectric power.--Electrical power generated by using fossil-fuel (coal, oil, or natural gas), geothermal, or nuclear energy.

Thermoelectric power water use.--Water used in the process of the generation of electric power. The majority of water used for this category is for cooling purposes (much of which is used for once-through cooling). Water is also used for boiler makeup or domestic purposes throughout the plant. Boiler makeup water and water used for domestic purposes are generally obtained from public supply, however for plants located in remote areas, this water can be self-supplied. Cooling water is generally self-supplied, although some smaller plants use public-supply water for cooling purposes.

Treated (wastewater) effluent.--Refers to water that has received primary, secondary, or advanced treatment and is released from a wastewater facility after treatment.

Wastewater.--A combination of liquid and water-carried pollutants from residential or commercial buildings, industrial plants, and institutions. Wastewater may include any ground water, surface runoff, or leachate that may be present in the system.

Water transfer---Artificial conveyance of water from one area to another across a political or hydrological boundary. This is referred to as an import or export of water from one basin or county to another. 
Water use.--The amount of water needed or used for a variety of purposes including drinking, irrigation, processing of goods, power generation, and other uses. The amount of water used may not equal the amount of water withdrawn due to water transfers or the recirculation or recycling of the same water. For example, a power plant may use the same water multiple times but withdrawal a significantly different amount.

Withdrawal.--Water removed from the ground or diverted from a surface-water source. 


\title{
Water-Use by Category, County, and Water Management District in Florida, 1950-90
}

\author{
By Richard L. Marella
}

\section{Abstract}

The population for Florida in 1990 was estimated at 12.94 million, an increase of nearly 10.17 million ( 370 percent) from the population of 2.77 million in 1950. Consequently, water use (fresh and saline) in Florida increased nearly 510 percent $(15,175$ million gallons per day $)$ between 1950 and 1990. The resident population of the State is projected to surpass 20 million by the year 2020. Through the cooperation of the Florida Department of Environmental Protection and the U.S. Geological Survey, water-use data for the period between 1950 and 1990 has been consolidated into one publication. This report aggregates and summarizes the quantities of water withdrawn annually for all water-use categories (public supply, self-supplied domestic, self-supplied commercial-industrial, agriculture, and thermoelectric power generation), by counties, and water management districts in Florida from 1950 through 1990.

Total water withdrawn in Florida increased from 2,923 million gallons per day in 1950 to 17,898 million gallons per day in 1990 . Surfacewater withdrawals during 1950 totaled 2,333 million gallons per day but were not differentiated between fresh and saline, therefore, comparisons between fresh and saline water were made beginning with 1955 data. Freshwater withdrawals increased 245 percent between 1955 and 1990. Saline water withdrawals increased more than 1,500 percent between 1955 and 1990. In 1955 , more than 47 percent of the freshwater used was withdrawn from groundwater sources and 53 percent was withdrawn from surface-water sources. In 1990, nearly 62 percent of the freshwater withdrawn was from groundwater sources, while 38 percent was withdrawn from surface-water sources. The steady increase in ground-water withdrawals since the 1950's primarily is a result of the ability to drill and pump water more economically from large, deep wells and the reliability of both the quality and quantity of water from these wells.

Water withdrawn for public supply in Florida increased 1,030 percent between 1950 and 1990. The population served by public supply increased from 1.66 million in 1950 to 11.23 million in 1990, and the percentage of the population served by public supply increased from 60 percent in 1950 to nearly 88 percent in 1990. Freshwater withdrawn for self-supplied domestic use in Florida increased 1,010 percent, self-supplied commercial-industrial uses increased 170 percent, and agriculture increased 915 percent between 1950 and 1990. Freshwater withdrawals for thermoelectric power generation decreased 8 percent between 1955 and 1990, while saline water withdrawals increased nearly 1,540 percent between 1955 and 1990.

Between 1965 and 1990, total freshwater withdrawals increased in 58 of the 67 counties in Florida. Fresh ground-water was withdrawn in all 67 counties in 1965 through 1990, and increased in 65 counties between 1965 and 1990. Fresh surface-water was withdrawn in 60 counties from 1965 to 1990 , and increased in 42 counties between 1965 and 1990. The change in total freshwater withdrawals within the water management districts between 1975 and 1990 were as follows; Northwest Florida Water 
Management District increased 3 percent, St. Johns River Water Management District decreased 6 percent, South Florida Water Management District increased 37 percent, Southwest Florida Water Management District decreased 1 percent, and Suwannee River Water Management District increased 8 percent.

\section{INTRODUCTION}

The resident population for Florida in 1990 was nearly 12.94 million (Smith, 1991). This is an increase of nearly 10.17 million (370 percent) from the 1950 population of 2.77 million (Dietrich, 1978), and is projected to surpass 20 million by the year 2020 (Smith and Bayya, 1991) (fig. 1). In addition to the resident population, an estimated 41 million non-resident visitors came to Florida during 1990 (Florida Division of Tourism, 1990). Agricultural production for Florida during 1990 was ranked ninth in the nation (U.S. Department of Agriculture, 1991). Due to the increase in population (residential and non-residential) and the development of large scale irrigation and crop production, water use (fresh and saline) in Florida increased nearly $15,175 \mathrm{Mgal} / \mathrm{d}$ (more than 510 percent) between 1950 and 1990. Combined, fresh and saline water use in 1950 totaled nearly $2,923 \mathrm{Mgal} / \mathrm{d}$ (MacKichan, 1951, p. 6) compared to 17,898 Mgal/d in 1990 (Marella, 1992b, p. 7).

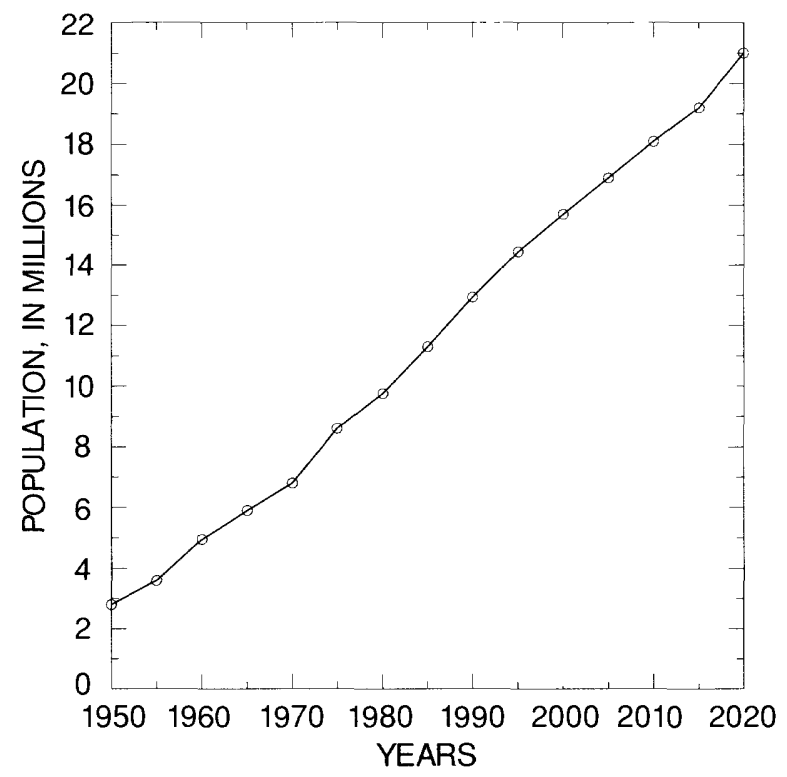

Figure 1. Population of Florida, 1950-2020 (from Dietrich, 1978, Smith and Bayya, 1991, and University of Florida, 1976, 1986, and 1991).
A general knowledge of water supply for Florida is vital to the support of the population, tourism, and agriculture. Information regarding the amount of water withdrawn, including where and how it is used, is needed to plan for future water demands in Florida. Water-use data are available in several reports but some data have not been previously published. The U.S. Geological Survey (USGS) in cooperation with the Florida Department of Environmental Protection (FDEP) (formerly the Florida Department of Environmental Regulation) aggregated and summarized into one publication the water-use data for Florida during the period between 1950 and 1990.

\section{Purpose and Scope}

The purpose of this report is to provide a summary of the aggregated water-use data that have been collected for Florida. This compilation presents the quantities of water withdrawn for all water-use categories, counties, and water management districts between 1950 and 1990. This information can be used by water managers and planners to determine trends in water use, establish water budgets, and project future water demands in Florida.

This report presents water-use data in Florida collected for each of the following categories: public supply, self-supplied domestic, self-supplied commercial-industrial (including mining uses), agriculture (including irrigation, livestock and fish farming uses), and thermoelectric power generation. Withdrawal data are presented for ground and surface water, as well as for fresh and saline sources. Water-use data (the amount of water withdrawn and used) are included for all 67 counties in Florida (fig. 2) and all five water management districts. Information concerning instream (nonwithdrawal) water use such as navigation, water-based recreation, propagation of fish and wildlife, water used for hydroelectric power generation, and dilution and conveyance of liquid or solid wastes, is not included. Additionally, information on consumptive use and domestic and industrial wastewater discharges is not included.

Water-use totals for each category are summarized for every five year period, beginning with 1950, and additional State summaries were made for all or some categories in 1977, 1979, 1986, 1987, 1988, and 1989. Water-use data that were compiled on a county level are presented for all years beginning in 1965. Water-use data that were summarized on a water 
management district level are presented for all years beginning in 1975. Data for counties and water management districts are subdivided by fresh ground- and surface-water totals.

Some water-use values have been adjusted or modified to reflect new or updated information that was obtained or verified for this report. These updates are noted on the individual county tables. However, because of these modifications, the county and State totals may not precisely match those that have been previously published. Overall, only 1 percent of the nearly 8,000 pieces of county water-use data were modified and reflect a more accurate water-use total for each county's as well as Florida.

Water withdrawals in this report are expressed in million gallons per day. Reported values are the average daily quantities used, as derived from annual data. The tables in this report list values in million gallons per day and are reported to two decimal places or to 10,000 gallons per day. Water-use values in the text, however, are rounded to whole numbers.

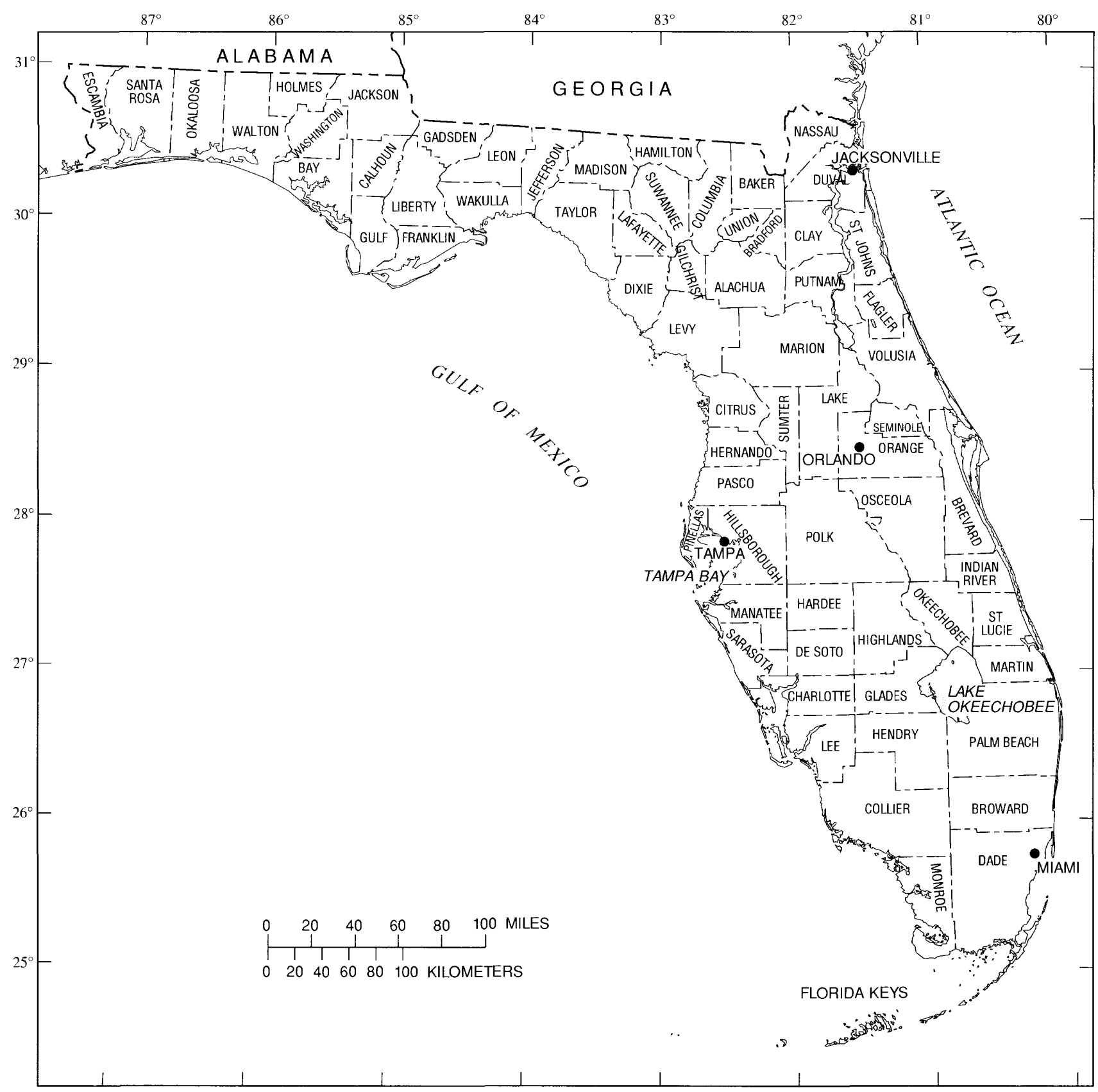

Figure 2. Counties and selected place names in Florida. 


\section{History of Water-Use Data Collection in Florida}

Water-use data for the early years of 1950 to 1965 were often collected on an intermittent basis by the USGS or the Florida Bureau of Geology (FBOG). Data were obtained from utility operators, plant managers, and farmers by field technicians that were usually involved in other types of data collection (such as water levels or stream flows) or obtained by staff engineers and hydrologists that were conducting studies in a specific area for a certain time frame. The State agency that managed the water resources during this time (The Florida Department of Water Resources) had no ongoing water-use data collection program. By 1970, water use in Florida had increased to such an extent that the USGS and the FBOG initiated a more formalized joint effort to collect and compile water-use data for the State. In 1972, the Florida Water Resource Act, Chapter 373 of the Florida Statutes, created five water management districts to manage the water resources of Florida on a regional level (Fernald and Patton, 1984, p. 132). Additionally, in the late 1970's, the U.S. Congress recognized the need for consistent water-use data and mandated that the USGS collect and compile this data on a county and hydrologic-unit level for each State. This brought a major change to water-use data collection efforts in Florida as federal funding became available to State agencies (water management districts) with an interest in collecting and compiling such data. By 1980, each water management district had personnel on staff for the purpose of collecting and compiling water-use data on a regular basis. However, by the mid 1980's, direct federal funding for water-use was discontinued. Subsequently, water management districts water-use data collection efforts became fragmented and sporadic.

Several districts opted to continue collecting and compiling data on a periodic basis, while others did little or no data collection or compilations on a regular bases. Also, the role of the USGS changed as the federal funding for water-use data collection was reduced. In the late 1980's, the Florida Department of Environmental Regulation (FDER) realized the need to reestablish the collection and compilation of water-use data on a consistent basis and established a cooperative program with the USGS. In this ongoing statewide program, the USGS coordinates with the five water management districts to collect, compile, and publish data for Florida on a five-year basis. In the 1990 's, the water-use program continues as a cooperative program between the Florida Department of
Environmental Protection and the USGS. The emphasis of this statewide program is to improve the consistency and continuity of water-use data throughout the State and to publish data for Florida on a fiveyear basis.

\section{Previous Investigations}

This study is the first compilation of all the existing water-use data (quantities of water withdrawn or used) in Florida. Statewide water-use summaries were published for Florida for 1965 (Pride, 1975), for 1970 (Pride, 1973); for 1975 (Leach, 1978a), for 1977 (Leach and Healy, 1980), for 1980 (Leach, 1983), for 1985 (Marella, 1988a), and for 1990 (Marella, 1992b). These reports included assessments of all water uses in Florida by county, including; public-supply, selfsupplied domestic, self-supplied commercialindustrial, agriculture, and thermoelectric power generation water uses. Prior to 1965 , State water-use data were published only at the National level. Water-use data for 1950 (MacKichan, 1951), for 1955 (MacKichan, 1957), and for 1960 (MacKichan and Kammerer, 1961) were published for the entire United States, but included only detailed water-use values by category at the State level, with no county data. Data for Florida also were presented in the National compilations for 1965 (Murray, 1968), 1970 (Murray and Reeves, 1972), 1975 (Murray and Reeves, 1977), 1980 (Solley and others, 1983), 1985 (Solley and others, 1988), and 1990 (Solley and others, 1993).

Water-use totals were summarized by water management district and published for 1975 (Leach, 1978a), 1977 (Leach and Healy, 1980), 1980 (Leach, 1983), and 1990 (Marella, 1992b). Water-use data for these and other years also were published by either the water management district or the USGS. These reports include: the Northwest Florida Water Management District water-use data for 1975, 1977, and 1980 (Kranzer, 1983) and 1985 (Bielby, 1987); the St. Johns River Water Management District water-use data for 1978 (Scott, 1980), 1979 (Marella, 1981), 1980 (Marella, 1987a), 1981 (Marella, 1983b), 1982 (Marella, 1984a), 1983 (Marella, 1984c), 1984 (Marella, 1985), 1985 (Marella, 1986), 1986 (Marella, 1988b), 1987 (Marella, 1990a), 1988 (Florence, 1990), 1989 (Florence, 1991), and 1990 (Florence, 1992); the South Florida Water Management District water-use data for 1980 (Woehlcke and others, 1982); the Southwest Florida Water Management District water-use 
data for 1979 (Duerr and Trommer, 1981a), 1980 (Duerr and Trommer, 1981b), 1981 (Duerr and Sohm, 1983), 1982 (Southwest Florida Water Management District, 1984), 1983 (Stieglitz, 1985a), 1984 (Stieglitz, 1985b), 1985 (Stieglitz, 1986), 1986 (Stieglitz and Tomik, 1987), 1987 (Tuttell and Sorensen, 1989), 1988 (Sorensen and others, 1990), and 1989-1990 (Sorensen, 1992), and; the Suwannee River Water Management District water-use data for 1977 (Suwannee River Water Management District, 1979). These reports detail water-use data for the counties and parts of the counties within each water management district. Because of differences in the data collection procedures, categories, data sources and methodologies used in these reports, the values reported by these agencies may not precisely equal those reported in this publication.

\section{Data Sources, Reliability, and Modifications}

Since the inception of this water-use compilation program in 1950, information on water-use has been collected or tabulated by eight different agencies in Florida. These agencies include The Florida Bureau of Geology, the Florida Department of Environmental Protection, the Northwest Florida Water Management District (NWFWMD), the St. Johns River Water Management District (SJRWMD), the South Florida Water Management District (SFWMD), the Southwest Florida Water Management District (SWFWMD), the Suwannee River Water Management District (SRWMD), and the USGS (Marella, 1988a, p. 10). Because these many agencies were involved in collecting and compiling water-use data between 1950 and 1990, the accuracy of the reported values varies from category to category and year to year. During this 40 year period, data collection and estimation methods have improved, along with an increase in the availability of data. Inherently, the accuracy of wateruse data has increased over time, as the 1990 data are believed to be more accurate than the data that were collected for 1980, and the 1980 data are believed to be more accurate than the 1970 data, and so on. Additionally, in Florida, as well as nationally, wateruse data for some categories are more accurate than others. For example, public-supply values are usually more accurate because most public-supply systems meter their usage, as opposed to agriculture or selfsupplied domestic values which are often estimated, because they generally are not metered. Water-use data for commercial, industrial, mining, and power facilities also are considered accurate, because most of these users meter their usage in Florida.

Some water-use values have been adjusted or modified from previously published data. These changes were made based on new or updated information obtained or verified for this report and are noted on the individual county tables. Overall, the modifications lowered the total freshwater withdrawal estimates for Florida about 3 percent from the previously published values for the 1970's (1970, 1975, and 1977). Freshwater withdrawal estimates for 1980 decreased nearly 10 percent from the previously published values, however, more than 80 percent of this decrease was the result of reclassifying the water source for several thermoelectric power plants from fresh to saline. Freshwater withdrawal estimates for 1985 changed less than 1 percent $(0.1$ percent), while the 1990 values did not change at all. The modifications can be classified into several categories. For public supply, most modifications were a result of eliminating the previous double counting of water withdrawals for several utilities in some counties or adding utilities that were missed in the original inventory. For commercial or industrial, most modifications were a result of adding missing systems, reclassifying cooling water under thermoelectric, or adjusting water withdrawals for heat exchange that are entirely returned to the source. For agriculture, most of the changes were a result of adjusting for the overestimation of the 1965 data (Leach, 1983). For thermoelectric power generation, most of the modifications were a result of changing reported self-supplied ground-water withdrawals to deliveries from public supply and reclassifying the water source of several withdrawals from fresh to saline water. These changes are detailed further in the individual category sections.

\section{Acknowledgments}

The author gratefully acknowledges the Florida Department of Environmental Protection for its cooperation in the State water-use program and to the five water management districts (Northwest Florida Water Management District, St. Johns River Water Management District, South Florida Water Management District, Southwest Florida Water Management District, and the Suwannee River Water Management District) for their continuing cooperation in collecting and providing water-use data. Additionally, special thanks are extended to the many utility operators, 
plant managers, and individuals who provided data vital to the completion of this effort. Some of these individuals include; George Cook and Addison Bennett (Bay County Water Department), Ronald Hix and Mark McLean (Florida Power and Light), Jacqueline Gray (Florida Power Corporation), Gary Wiess and William Essex (City of Jacksonville), and the many other individuals who provided data or technical guidance. The author would also like to thank A.D. Duerr of the U.S. Geological Survey for his input and data verification for this report.

\section{WATER USE IN FLORIDA, 1950-90}

Total water withdrawn in Florida increased from $2,923 \mathrm{Mgal} / \mathrm{d}$ in 1950 to $17,898 \mathrm{Mgal} / \mathrm{d}$ in 1990 (table 1). Surface-water withdrawals for 1950 totaled 2,333 Mgal/d, but were not differentiated between fresh and saline, therefore, comparisons between fresh and saline water were made from 1955. Freshwater withdrawals have increased 245 percent between 1955 (2,185 Mgal/d) and 1990 (7,532 Mgal/d) (fig. 3 and table 1). Saline water withdrawals increased more than 1,500 percent between 1955 (645 Mgal/d) and 1990 (10,366 Mgal/d) (fig. 3 and table 1). Between 1980 and 1990, freshwater withdrawals increased 14 percent and saline water withdrawals decreased 28 percent. However, the reader is cautioned that the early water-use values for the 1950's and 1960's are not as accurate as the values for the 1980's and 1990's.

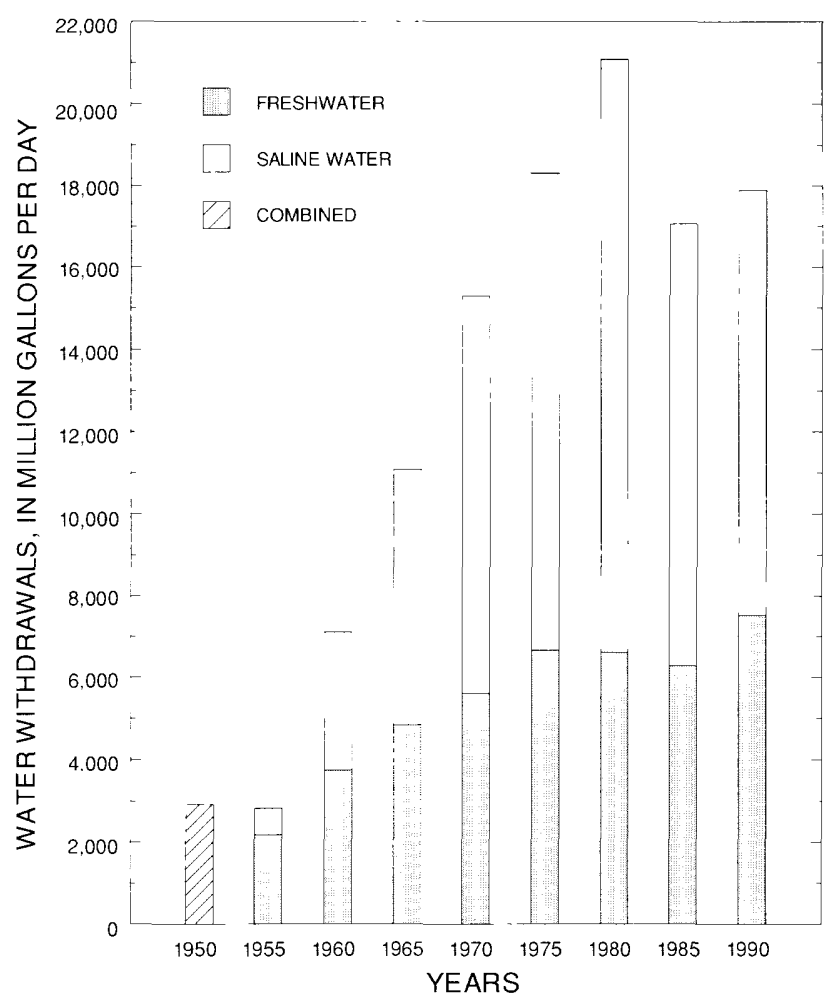

Figure 3. Total water withdrawn in Florida between 1950 and 1990.

Freshwater withdrawals in Florida were first reported by county for 1965 . Total freshwater withdrawals in Florida increased in 58 of 67 counties between 1965 and 1990. The greatest increase occurred in Gilchrist County (2,120 percent) while the greatest decrease occurred in Levy County (45 percent) (table 2). From 1980 to 1990 total freshwater withdrawals increased in 50 counties and decreased in 17 counties.

Table 1. Total water withdrawals in Florida by source, 1950-90

[All withdrawal values are in million gallons per day; modified from Dietrich (1978), Leach (1978a, 1983), Leach and Healy (1980), MacKichan (1951, 1957), MacKichan and Kammmerer (1961), Marella (1988a, 1992b). Murray (1968), Murray and Reeves (1972), Pride (1973), University of Florida (1976, 1986, 1991)]

\begin{tabular}{|c|c|c|c|c|c|c|c|c|}
\hline \multirow[b]{2}{*}{ Year } & \multirow{2}{*}{$\begin{array}{c}\text { Florida } \\
\text { population } \\
\text { (in millions) }\end{array}$} & \multicolumn{3}{|c|}{ Freshwater withdrawn by source } & \multicolumn{3}{|c|}{ Saline water withdrawn by source } & \multirow{2}{*}{$\begin{array}{c}\text { Total } \\
\text { withdrawn }\end{array}$} \\
\hline & & $\begin{array}{c}\text { Ground } \\
\text { water }\end{array}$ & $\begin{array}{c}\text { Surface } \\
\text { water }\end{array}$ & Totals & $\begin{array}{c}\text { Ground } \\
\text { water }\end{array}$ & $\begin{array}{c}\text { Surface } \\
\text { water }\end{array}$ & Totals & \\
\hline 1950 & 2.8 & 590.0 & $2,333.0^{\mathrm{a}}$ & $0.0^{\mathrm{a}}$ & 0.0 & $0.0^{\mathrm{a}}$ & $0.0^{\mathrm{a}}$ & $2,923.0^{\mathrm{a}}$ \\
\hline 1955 & 3.6 & $1,035.0$ & $1,150.0$ & $2,185.0$ & 15.0 & 630.0 & 645.0 & $2,830.0$ \\
\hline 1960 & 5.0 & $1,560.0$ & $2,198.5$ & $3,758.5$ & 0.0 & $3,360.0$ & $3,360.0$ & $7,118.5$ \\
\hline 1965 & 5.9 & $2,218.5^{\mathrm{b}}$ & $2,623.3^{b}$ & $4,841.8$ & 80.2 & $6,161.0$ & $6,241.2$ & $11,083.0$ \\
\hline 1970 & 6.8 & $2,786.7^{b}$ & $2,825.6^{\mathrm{b}}$ & $5,612.3$ & 137.0 & $9,545 \cdot 6^{\mathrm{b}}$ & $9,682.6$ & $15,294.9$ \\
\hline 1975 & 8.5 & $3,214.6^{\mathrm{b}}$ & $3,453.6^{b}$ & $6,668.2$ & 95.5 & $11,551.1^{b}$ & $11,646.6$ & $18,314.8$ \\
\hline 1977 & 8.9 & $3,429.5^{b}$ & $3,023.5^{\mathrm{b}}$ & $6,453.0$ & 108.5 & $14,796.5^{b}$ & $14,905.0$ & $21,358.0$ \\
\hline 1980 & 9.8 & $3,677.2^{b}$ & $2,937.3^{b}$ & $6,614.5$ & 121.2 & $14,349 \cdot 6^{b}$ & $14,470.8$ & $21,085.3$ \\
\hline 1985 & 11.3 & $4,047.7^{\mathrm{C}}$ & $2,236.6^{b}$ & $6,284.3$ & 59.1 & $10,721.9$ & $10,781.0$ & $17,065.3$ \\
\hline 1990 & 12.9 & $4,664.7$ & $2,867.1$ & $7,531.8$ & 49.3 & $10,316.9$ & $10,366.2$ & $17,898.0$ \\
\hline
\end{tabular}

${ }^{\mathrm{a}}$ Fresh and saline water withdrawal totals were not delineated for 1950.

${ }^{b}$ Water withdrawal value has been adjusted from the originally published numbers.

${ }^{c}$ Includes ground-water withdrawn from nonpotable sources that was reported as saline water. 
Table 2. Freshwater withdrawn and percent change in Florida by county, 1965-90

[Modified from Pride (1973), Leach (1978a, 1983), Marella (1988a, 1992b); values may not add to totals because of independent rounding or revisions in data]

\begin{tabular}{|c|c|c|c|c|c|c|c|c|}
\hline \multirow{2}{*}{ County } & \multicolumn{6}{|c|}{ Freshwater withdrawn, in million gallons per day } & \multicolumn{2}{|c|}{ Percent change } \\
\hline & 1965 & 1970 & 1975 & 1980 & 1985 & 1990 & $1965-90$ & $1980-90$ \\
\hline Alachua & 26.4 & 34.8 & 32.9 & 48.5 & 51.5 & 52.5 & 99 & 8 \\
\hline Baker & 0.7 & 1.3 & 3.3 & 5.3 & 8.3 & 10.1 & 1,343 & 91 \\
\hline Bay & 41.4 & 45.4 & 37.7 & 43.9 & 50.2 & 58.2 & 41 & 33 \\
\hline Bradford & 0.9 & 3.4 & 5.9 & 8.8 & 9.3 & 9.1 & 911 & 3 \\
\hline Brevard & 77.8 & 62.9 & 78.2 & 191.1 & 155.5 & 144.5 & 86 & -24 \\
\hline Broward & 173.2 & 184.4 & 229.5 & 235.6 & 235.3 & 266.6 & 54 & 13 \\
\hline Calhoun & 3.0 & 1.0 & 2.6 & 3.4 & 1.7 & 2.4 & -20 & -29 \\
\hline Charlotte & 7.8 & 31.9 & 37.9 & 28.6 & 54.2 & 50.7 & 550 & 77 \\
\hline Citrus & 3.1 & 7.4 & 6.4 & 12.0 & 25.9 & 25.9 & 735 & 116 \\
\hline Clay & 8.4 & 9.5 & 18.2 & 20.4 & 26.6 & 25.6 & 205 & 25 \\
\hline Collier & 56.7 & 54.9 & 82.9 & 111.1 & 123.7 & 213.8 & 277 & 92 \\
\hline Columbia & 2.3 & 2.9 & 4.7 & 8.0 & 9.9 & 11.4 & 396 & 43 \\
\hline Dade & 287.8 & 276.5 & 374.0 & 440.5 & 490.6 & 541.0 & 88 & 23 \\
\hline De Soto & 27.6 & 68.1 & 71.3 & 40.2 & 83.8 & 122.0 & 342 & 203 \\
\hline Dixie & 0.6 & 1.9 & 4.8 & 1.8 & 4.3 & 5.9 & 883 & 228 \\
\hline Duval & 128.3 & 168.9 & 159.9 & 142.8 & 160.7 & 154.4 & 20 & 8 \\
\hline Escambia & 319.9 & 322.5 & 379.6 & 430.0 & 343.1 & 292.2 & -9 & -32 \\
\hline Flagler & 4.0 & 9.7 & 9.4 & 5.5 & 9.8 & 14.7 & 268 & 167 \\
\hline Franklin & 0.9 & 0.9 & 1.1 & 1.3 & 3.0 & 2.7 & 200 & 108 \\
\hline Gadsden & 10.9 & 9.5 & 9.1 & 13.5 & 16.2 & 18.5 & 70 & 37 \\
\hline Gilchrist & 0.5 & 0.6 & 1.1 & 2.7 & 5.4 & 11.1 & 2,120 & 311 \\
\hline Glades & 9.9 & 47.2 & 54.1 & 125.7 & 82.1 & 81.8 & 726 & -35 \\
\hline Gulf & 28.8 & 37.2 & 35.2 & 35.5 & 37.9 & 45.2 & 57 & 27 \\
\hline Hamilton & 11.9 & 20.0 & 33.1 & 38.0 & 43.4 & 50.6 & 325 & 33 \\
\hline Hardee & 46.2 & 64.3 & 97.1 & 43.6 & 94.2 & 66.6 & 44 & 53 \\
\hline Hendry & 252.8 & 248.8 & 297.3 & 248.3 & 192.0 & 493.0 & 95 & 99 \\
\hline Hernando & 30.0 & 23.3 & 31.9 & 41.6 & 40.9 & 45.9 & 53 & 10 \\
\hline Highlands & 181.2 & 289.4 & 247.6 & 176.2 & 123.7 & 141.5 & -22 & -20 \\
\hline Hillsborough & 203.7 & 215.4 & 185.1 & 218.0 & 317.2 & 268.6 & 32 & 23 \\
\hline Holmes & 0.9 & 1.3 & 1.5 & 2.8 & 5.4 & 6.8 & 656 & 143 \\
\hline Indian River & 65.7 & 136.8 & 310.2 & 294.4 & 152.2 & 190.4 & 190 & -35 \\
\hline Jackson & 119.1 & 151.2 & 132.2 & 135.8 & 129.3 & 141.5 & 19 & 4 \\
\hline Jefferson & 0.9 & 1.8 & 2.5 & 2.5 & 9.6 & 12.1 & 1,244 & 384 \\
\hline Lafayette & 0.6 & 1.4 & 3.2 & 2.6 & 7.6 & 9.6 & 1,500 & 269 \\
\hline Lake & 55.4 & 53.8 & 92.5 & 101.1 & 73.2 & 97.1 & 75 & -4 \\
\hline Lee & 35.1 & 49.6 & 91.6 & 87.3 & 79.7 & 161.4 & 360 & 85 \\
\hline Leon & 11.2 & 15.1 & 21.1 & 29.2 & 36.6 & 39.5 & 253 & 35 \\
\hline Levy & 44.2 & 2.0 & 3.7 & 5.6 & 18.6 & 24.5 & -45 & 338 \\
\hline Liberty & 1.1 & 1.7 & 0.7 & 0.7 & 1.1 & 3.7 & 236 & 429 \\
\hline Madison & 1.8 & 3.2 & 4.0 & 4.5 & 6.0 & 7.5 & 317 & 67 \\
\hline Manatee & 46.7 & 66.3 & 77.1 & 103.1 & 127.5 & 141.8 & 204 & 38 \\
\hline Marion & 19.7 & 17.8 & 32.1 & 37.0 & 53.0 & 51.1 & 159 & 38 \\
\hline Martin & 59.1 & 94.3 & 92.3 & 191.9 & 189.2 & 209.6 & 255 & 9 \\
\hline Monroe & 0.2 & 1.6 & 1.8 & 3.0 & 1.6 & 1.3 & 550 & -57 \\
\hline Nassau & 40.4 & 53.4 & 63.1 & 54.6 & 47.0 & 43.4 & 7 & -21 \\
\hline Okaloosa & 11.3 & 15.6 & 18.5 & 21.7 & 27.7 & 31.6 & 180 & 46 \\
\hline Okeechobee & 39.4 & 35.8 & 87.0 & 91.5 & 29.7 & 44.3 & 12 & -52 \\
\hline Orange & 185.5 & 228.5 & 211.6 & 203.2 & 245.7 & 282.4 & 52 & 39 \\
\hline Osceola & 20.2 & 18.3 & 19.7 & 31.7 & 59.3 & 76.8 & 280 & 142 \\
\hline Palm Beach & 414.5 & 504.4 & 659.8 & 752.7 & 708.2 & 996.8 & 140 & 32 \\
\hline Pasco & 35.0 & 47.8 & 104.4 & 98.3 & 126.9 & 141.1 & 303 & 44 \\
\hline Pinellas & 46.5 & 51.1 & 79.0 & 50.3 & 24.1 & 49.5 & 6 & -2 \\
\hline Polk & 624.5 & 625.2 & 713.4 & 610.8 & 398.8 & 437.5 & -30 & -28 \\
\hline Putnam & 86.3 & 169.6 & 181.8 & 85.8 & 90.4 & 80.2 & -7 & -7 \\
\hline St. Johns & 17.6 & 26.3 & 35.9 & 31.3 & 51.3 & 52.7 & 199 & 68 \\
\hline St. Lucie & 191.4 & 192.9 & 379.2 & 246.9 & 231.9 & 254.5 & 33 & 3 \\
\hline Santa Rosa & 10.2 & 15.7 & 22.7 & 26.0 & 16.9 & 23.8 & 133 & -8 \\
\hline Sarasota & 49.0 & 50.3 & 40.3 & 35.0 & 40.9 & 62.7 & 28 & 79 \\
\hline Seminole & 16.9 & 15.6 & 32.1 & 60.5 & 68.8 & 67.4 & 299 & 11 \\
\hline Sumter & 31.5 & 25.1 & 22.2 & 21.3 & 78.7 & 73.6 & 134 & 246 \\
\hline Suwannee & 182.2 & 185.8 & 179.4 & 185.8 & 87.9 & 141.8 & -22 & -24 \\
\hline Taylor & 51.4 & 55.4 & 59.2 & 53.6 & 50.1 & 50.7 & -1 & -5 \\
\hline Union & 1.3 & 1.6 & 1.7 & 1.3 & 2.1 & 5.1 & 292 & 292 \\
\hline Volusia & 373.3 & 437.1 & 351.3 & 215.3 & 196.9 & 277.6 & -26 & 29 \\
\hline Wakulla & 1.3 & 2.1 & 2.3 & 2.2 & 1.3 & 2.7 & 108 & 23 \\
\hline Walton & 1.6 & 12.8 & 3.2 & 12.5 & 6.6 & 9.0 & 463 & -28 \\
\hline Washington & 1.1 & 1.3 & 1.6 & 2.0 & 3.2 & 3.4 & 209 & 70 \\
\hline Totals & $4,841.7$ & $5,612.3$ & $6,668.2$ & $6,614.5$ & $6,284.3$ & $7,531.8$ & 56 & 14 \\
\hline
\end{tabular}




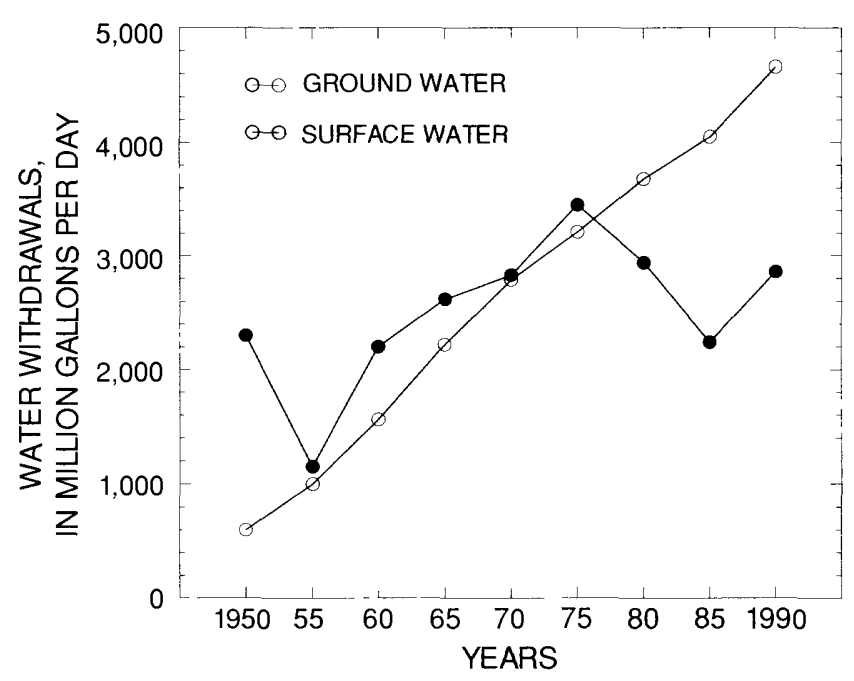

Figure 4. Freshwater withdrawn in Florida by source, 1950 90 (surface-water value for 1950 may include some saline water; modified from Marella, 1992b).

In 1990 , nearly 62 percent $(4,665 \mathrm{Mgal} / \mathrm{d})$ of the freshwater withdrawn was from ground-water sources, while 38 percent was from surface-water sources. In 1955 , more than 47 percent $(1,035 \mathrm{Mgal} / \mathrm{d})$ was withdrawn from ground-water sources and 53 percent $(1,150 \mathrm{Mgal} / \mathrm{d})$ was withdrawn from surface-water sources (table 1). Fresh ground-water withdrawals increased nearly 350 percent from $1,035 \mathrm{Mgal} / \mathrm{d}$ in 1955 to $4,665 \mathrm{Mgal} / \mathrm{d}$ in 1990 (fig. 4 and table 1). The steady increase in ground-water withdrawals since the 1950 's primarily has been a result of the ability to drill and pump water more economically from large, deep wells and the reliability of both the quality and quantity of water from these wells. Fresh ground-water was withdrawn in all 67 counties from 1965 through 1990, and increased in 65 counties between 1965 and 1990 (table 3). Fresh surface-water was withdrawn in 60 counties in 1965 and 1990, and increased in 42 counties between 1965 and 1990. This is an increase of nearly 150 percent from $1,150 \mathrm{Mgal} / \mathrm{d}$ in 1955 to $2,867 \mathrm{Mgal} / \mathrm{d}$ in 1990 (fig. 4 and table 1). The increase in surface water use resulted in part, to the draining of wetlands for development for agricultural and (or) urban purposes and the impoundment of the diverted water for flood control as a source of irrigation water, and in part, to the operation of several new thermoelectric power plants that needed surface water for cooling purposes during this period. However, between 1980 and 1990, surface water withdrawals have decreased about 2 percent (fig. 4 and table 1). The decline in fresh surface-water withdrawals between 1980 and 1990 primarily has been a result of a decrease in thermoelectric power generation withdrawals. More than 1,000 Mgal/d of the decrease reflects a change in the water used for once-through cooling water for thermoelectric power generation (Marella, 1992b). Fresh surface-water was withdrawn in 60 counties in 1965 and 1990, and increased in 42 counties between 1965 and 1990 (table 4).

Between 1950 and 1990, freshwater withdrawals for public supply increased 1,030 percent (1,755 Mgal/d), self-supplied domestic use increased 1,010 percent (272 Mgal/d), self-supplied commercialindustrial use increased 169 percent ( $384 \mathrm{Mgal} / \mathrm{d})$, and agricultural use increased 915 percent $(3,430 \mathrm{Mgal} / \mathrm{d})$ (fig. 5). Withdrawals for thermoelectric power generation were not delineated between fresh and saline for 1950 , therefore 1955 values were used. Total freshwater withdrawals for thermoelectric power generation decreased 8 percent (68 Mgal/d) from 1955 to 1990 , while total saline water withdrawals for thermoelectric power generation increased nearly 1,540 percent (9,680 Mgal/d). Between 1980 and 1990, freshwater withdrawals for public supply increased 37 percent (520 Mgal/d), self-supplied domestic use increased 23 percent $(56 \mathrm{Mgal} / \mathrm{d})$, self-supplied commercialindustrial use increased 10 percent $(70 \mathrm{Mgal} / \mathrm{d})$, and agricultural use increased 26 percent $(780 \mathrm{Mgal} / \mathrm{d})$. Total freshwater withdrawals for thermoelectric power generation decreased nearly 41 percent $(507 \mathrm{Mgal} / \mathrm{d})$ and saline water withdrawals decreased 28 percent (4,074 Mgal/d). Since 1980, ground water was the primary source for public supply, domestic self-supplied, self-supplied commercial-industrial, and agriculture withdrawals, while surface water was the primary source for thermoelectric power generation withdrawals (table 5). Detailed information on each category is presented in the next section.

\section{WATER USE IN FLORIDA BY CATEGORY, 1950-90}

Since 1950 , water-use data have been collected and compiled for the following general categories; public supply, self-supplied domestic, self-supplied commercial-industrial, agriculture (irrigation and nonirrigation), and thermoelectric power generation. From 1950 to 1980 , self-supplied domestic was called rural domestic, and included water used for self-supplied domestic and livestock watering purposes (nonirrigation). 
Table 3. Fresh ground-water withdrawn and percent change in Florida by county, 1965-90

[Modified from Pride (1973), Leach (1978a; 1983), Marella (1988a, 1992b); values for 1985 include saline water withdrawn for public supply; values may not add to totals because of independent rounding or revisions in data]

\begin{tabular}{|c|c|c|c|c|c|c|c|c|}
\hline \multirow{2}{*}{ County } & \multicolumn{6}{|c|}{ Fresh ground-water withdrawn, in million gallons per day } & \multicolumn{2}{|c|}{ Percent change } \\
\hline & 1965 & 1970 & 1975 & 1980 & 1985 & 1990 & $1965-90$ & $1980-90$ \\
\hline Alachua & 25.7 & 34.1 & 30.8 & 44.1 & 51.4 & 52.1 & 103 & 18 \\
\hline Baker & 0.7 & 1.3 & 2.6 & 3.8 & 6.1 & 7.9 & 1,029 & 108 \\
\hline Bay & 9.7 & 7.5 & 4.9 & 7.3 & 12.0 & 16.0 & 65 & 119 \\
\hline Bradford & 0.8 & 3.3 & 5.8 & 8.7 & 9.3 & 9.0 & 1,025 & 3 \\
\hline Brevard & 69.0 & 55.2 & 47.6 & 170.8 & 115.7 & 117.9 & 71 & -31 \\
\hline Broward & 109.6 & 142.6 & 168.5 & 224.5 & 230.2 & 244.8 & 123 & 9 \\
\hline Calhoun & 2.5 & 0.9 & 2.4 & 3.3 & 1.7 & 1.9 & -24 & -42 \\
\hline Charlotte & 6.6 & 30.2 & 36.1 & 23.7 & 47.7 & 44.0 & 567 & 86 \\
\hline Citrus & 1.9 & 7.3 & 6.2 & 11.6 & 25.2 & 25.6 & 1,247 & 121 \\
\hline Clay & 8.4 & 9.5 & 13.9 & 19.1 & 21.3 & 25.2 & 200 & 32 \\
\hline Collier & 50.6 & 53.6 & 77.1 & 107.5 & 118.2 & 195.3 & 286 & 82 \\
\hline Columbia & 2.2 & 2.9 & 4.4 & 7.2 & 9.8 & 11.2 & 409 & 56 \\
\hline Dade & 285.1 & 273.8 & 371.1 & 437.7 & 486.1 & 526.6 & 85 & 20 \\
\hline De Soto & 23.2 & 66.8 & 67.2 & 36.7 & 74.9 & 102.1 & 340 & 178 \\
\hline Dixie & 0.5 & 1.8 & 1.6 & 1.6 & 4.1 & 5.9 & 1,080 & 269 \\
\hline Duval & 127.2 & 168.9 & 159.5 & 141.4 & 159.3 & 153.0 & 20 & 8 \\
\hline Escambia & 62.2 & 74.6 & 81.7 & 72.6 & 84.3 & 82.3 & 32 & 13 \\
\hline Flagler & 4.0 & 9.5 & 9.4 & 5.4 & 8.9 & 13.5 & 238 & 150 \\
\hline Franklin & 0.9 & 0.9 & 1.1 & 1.3 & 3.0 & 2.6 & 189 & 100 \\
\hline Gadsden & 2.7 & 4.1 & 3.1 & 4.3 & 8.5 & 9.5 & 252 & 121 \\
\hline Gilchrist & 0.3 & 0.6 & 1.0 & 2.3 & 5.4 & 11.1 & 3,600 & 383 \\
\hline Glades & 0.6 & 6.1 & 12.1 & 18.4 & 16.0 & 19.2 & 3,100 & 4 \\
\hline Gulf & 0.3 & 0.8 & 1.3 & 1.7 & 4.0 & 7.1 & 2,267 & 318 \\
\hline Hamilton & 11.6 & 19.8 & 32.9 & 37.8 & 43.4 & 50.6 & 336 & 34 \\
\hline Hardee & 44.0 & 64.3 & 97.1 & 43.6 & 94.2 & 60.6 & 38 & 39 \\
\hline Hendry & 39.2 & 26.0 & 81.8 & 111.9 & 33.9 & 174.5 & 345 & 56 \\
\hline Hernando & 29.8 & 23.3 & 31.6 & 41.5 & 40.9 & 45.5 & 53 & 10 \\
\hline Highlands & 36.5 & 27.4 & 93.7 & 66.9 & 100.4 & 129.4 & 255 & 93 \\
\hline Hillsborough & 152.3 & 153.8 & 122.0 & 159.7 & 249.3 & 179.7 & 18 & 13 \\
\hline Holmes & 0.7 & 1.3 & 1.2 & 2.4 & 5.0 & 5.6 & 700 & 133 \\
\hline Indian River & 29.0 & 102.9 & 51.7 & 79.8 & 45.0 & 72.9 & 151 & -9 \\
\hline Jackson & 3.2 & 7.2 & 11.1 & 15.2 & 18.4 & 30.1 & 841 & 98 \\
\hline Jefferson & 0.8 & 1.7 & 2.4 & 2.4 & 8.2 & 10.3 & 1,188 & 329 \\
\hline Lafayette & 0.5 & 1.2 & 3.0 & 2.3 & 7.3 & 9.1 & 1,720 & 296 \\
\hline Lake & 43.1 & 46.1 & 73.2 & 93.5 & 64.9 & 84.4 & 96 & -10 \\
\hline Lee & 6.9 & 36.0 & 61.2 & 67.1 & 68.3 & 123.1 & 1,684 & 83 \\
\hline Leon & 10.8 & 15.1 & 20.8 & 29.0 & 34.4 & 39.0 & 261 & 34 \\
\hline Levy & 2.1 & 2.0 & 3.6 & 4.5 & 14.1 & 21.5 & 924 & 378 \\
\hline Liberty & 1.0 & 1.2 & 0.7 & 0.7 & 1.1 & 3.7 & 270 & 429 \\
\hline Madison & 1.3 & 3.1 & 3.8 & 3.4 & 6.0 & 7.2 & 454 & 112 \\
\hline Manatee & 41.6 & 56.7 & 30.8 & 70.5 & 93.2 & 96.5 & 132 & 37 \\
\hline Marion & 18.1 & 16.6 & 31.2 & 35.9 & 49.1 & 49.2 & 172 & 37 \\
\hline Martin & 14.6 & 19.4 & 15.5 & 44.6 & 46.3 & 55.7 & 282 & 25 \\
\hline Monroe & 0.2 & 1.6 & 1.8 & 3.0 & 1.6 & 1.3 & 550 & -57 \\
\hline Nassau & 40.3 & 53.4 & 63.1 & 53.7 & 46.1 & 42.8 & 6 & -20 \\
\hline Okaloosa & 11.1 & 15.6 & 18.0 & 21.5 & 27.7 & 31.6 & 185 & 47 \\
\hline Okeechobee & 32.9 & 29.3 & 68.9 & 76.6 & 24.4 & 38.9 & 18 & -49 \\
\hline Orange & 81.8 & 91.6 & 113.8 & 172.3 & 191.8 & 220.9 & 170 & 28 \\
\hline Osceola & 13.4 & 12.9 & 15.5 & 27.6 & 53.7 & 63.4 & 373 & 130 \\
\hline Palm Beach & 62.2 & 170.8 & 113.8 & 162.9 & 153.9 & 201.0 & 223 & 23 \\
\hline Pasco & 31.9 & 47.8 & 94.0 & 97.5 & 126.0 & 138.7 & 335 & 42 \\
\hline Pinellas & 46.3 & 51.1 & 79.0 & 49.8 & 23.5 & 48.1 & 4 & -3 \\
\hline Polk & 350.4 & 426.9 & 407.9 & 312.3 & 320.4 & 353.7 & 1 & 13 \\
\hline Putnam & 17.8 & 28.5 & 40.6 & 60.2 & 70.3 & 61.4 & 245 & 2 \\
\hline St. Johns & 15.8 & 25.2 & 35.8 & 30.8 & 50.2 & 51.3 & 225 & 67 \\
\hline St. Lucie & 52.1 & 33.4 & 59.9 & 63.8 & 68.3 & 83.8 & 61 & 31 \\
\hline Santa Rosa & 10.2 & 15.7 & 22.5 & 25.7 & 16.7 & 23.5 & 130 & -9 \\
\hline Sarasota & 48.0 & 47.7 & 38.0 & 33.2 & 40.4 & 59.5 & 24 & 79 \\
\hline Seminole & 10.9 & 12.9 & 32.1 & 60.1 & 66.7 & 65.6 & 502 & 9 \\
\hline Sumter & 31.3 & 25.1 & 22.0 & 21.0 & 78.3 & 73.1 & 134 & 248 \\
\hline Suwannee & 1.8 & 12.8 & 6.6 & 11.8 & 21.3 & 32.3 & 1,694 & 174 \\
\hline Taylor & 51.3 & 55.4 & 59.1 & 53.5 & 48.5 & 49.4 & -4 & -8 \\
\hline Union & 1.2 & 1.6 & 1.7 & 1.2 & 2.1 & 4.4 & 267 & 267 \\
\hline Volusia & 21.9 & 30.8 & 37.3 & 58.7 & 79.1 & 74.4 & 240 & 27 \\
\hline Wakulla & 0.3 & 1.7 & 1.8 & 2.1 & 1.3 & 2.7 & 800 & 29 \\
\hline Walton & 1.6 & 12.8 & 2.9 & 11.4 & 6.1 & 7.9 & 394 & -31 \\
\hline Washington & 0.8 & 1.3 & 1.5 & 1.9 & 3.1 & 3.3 & 313 & 74 \\
\hline Totals & $2,218.5$ & $2,786.7$ & $3,214.6$ & 3.677 .2 & $4,047.7$ & $4,664.7$ & 110 & 27 \\
\hline
\end{tabular}


Table 4. Fresh surface-water withdrawn and percent change in Florida by county, 1965-90

[Modified from Pride (1973), Leach (1978a, 1983), Marella (1988a. 1992b), values may not add to totals because of independent rounding or revisions in data: N/A, not applicable]

\begin{tabular}{|c|c|c|c|c|c|c|c|c|}
\hline \multirow{2}{*}{ County } & \multicolumn{6}{|c|}{ Fresh surface-water withdrawn, in million gallons per day } & \multicolumn{2}{|c|}{ Percent change } \\
\hline & 1965 & 1970 & 1975 & 1980 & 1985 & 1990 & $1965-90$ & $1980-90$ \\
\hline Alachua & 0.8 & 0.7 & 2.1 & 4.4 & 0.1 & 0.4 & -50 & -91 \\
\hline Baker & 0.0 & 0.0 & 0.7 & 1.5 & 2.2 & 2.2 & 2,200 & 47 \\
\hline Bay & 31.7 & 37.9 & 32.8 & 36.6 & 38.2 & 42.2 & 33 & 15 \\
\hline Bradford & 0.1 & 0.1 & 0.1 & 0.1 & 0.0 & 0.1 & 0 & 0 \\
\hline Brevard & 8.8 & 7.7 & 30.6 & 20.3 & 39.8 & 26.6 & 202 & 31 \\
\hline Broward & 63.6 & 41.8 & 61.0 & 11.1 & 5.1 & 21.8 & -66 & 96 \\
\hline Calhoun & 0.5 & 0.1 & 0.2 & 0.1 & 0.0 & 0.5 & 0 & 400 \\
\hline Charlotte & 1.2 & 1.7 & 1.8 & 4.9 & 6.5 & 6.7 & 458 & 37 \\
\hline Citrus & 1.2 & 0.1 & 0.2 & 0.4 & 0.7 & 0.3 & -75 & -25 \\
\hline Clay & 0.0 & 0.0 & 4.3 & 1.3 & 5.3 & 0.4 & 400 & -69 \\
\hline Collier & 6.1 & 1.3 & 5.8 & 3.6 & 5.5 & 18.5 & 203 & 414 \\
\hline Columbia & 0.1 & 0.0 & 0.3 & 0.8 & 0.1 & 0.2 & 100 & -75 \\
\hline Dade & 2.7 & 2.7 & 2.9 & 2.8 & 4.5 & 14.4 & 433 & 414 \\
\hline De Soto & 4.4 & 1.3 & 4.1 & 3.5 & 8.9 & 19.9 & 352 & 469 \\
\hline Dixie & 0.1 & 0.1 & 3.2 & 0.2 & 0.2 & 0.0 & -100 & -100 \\
\hline Duval & 1.1 & 0.0 & 0.4 & 1.4 & 1.4 & 1.4 & 27 & 0 \\
\hline Escambia & 257.7 & 247.9 & 297.9 & 357.4 & 258.8 & 209.9 & -19 & -41 \\
\hline Flagler & 0.0 & 0.2 & 0.0 & 0.1 & 0.9 & 1.2 & 100 & 1.100 \\
\hline Franklin & 0.0 & 0.0 & 0.0 & 0.0 & 0.0 & 0.1 & 100 & 100 \\
\hline Gadsden & 8.2 & 5.4 & 6.0 & 9.2 & 7.7 & 9.0 & 10 & -2 \\
\hline Gilchrist & 0.2 & 0.0 & 0.1 & 0.4 & 0.0 & 0.0 & -100 & -100 \\
\hline Glades & 9.3 & 41.1 & 42.0 & 107.3 & 66.1 & 62.6 & 573 & -42 \\
\hline Gulf & 28.5 & 36.4 & 33.9 & 33.8 & 33.9 & 38.1 & 34 & 13 \\
\hline Hamilton & 0.3 & 0.2 & 0.2 & 0.2 & 0.0 & 0.0 & -100 & -100 \\
\hline Hardee & 2.2 & 0.0 & 0.0 & 0.0 & 0.0 & 6.0 & 173 & 6,000 \\
\hline Hendry & 213.6 & 222.8 & 215.5 & 136.4 & 158.1 & 318.5 & 49 & 1.34 \\
\hline Hernando & 0.2 & 0.0 & 0.3 & 0.1 & 0.0 & 0.4 & 100 & 300 \\
\hline Highlands & 144.7 & 262.0 & 153.9 & 109.3 & 23.3 & 12.1 & -92 & -89 \\
\hline Hillsborough & 51.4 & 61.6 & 63.1 & 58.3 & 67.9 & 88.9 & 73 & 52 \\
\hline Holmes & 0.2 & 0.0 & 0.3 & 0.4 & 0.4 & 1.2 & 500 & 200 \\
\hline Indian River & 36.7 & 33.9 & 258.5 & 214.6 & 107.2 & 117.5 & 220 & -45 \\
\hline Jackson & 115.9 & 144.0 & 121.1 & 120.6 & 110.9 & 111.4 & -4 & -8 \\
\hline Jefferson & 0.1 & 0.1 & 0.1 & 0.1 & 1.4 & 1.8 & 1.700 & 1,700 \\
\hline Lafayette & 0.1 & 0.2 & 0.2 & 0.3 & 0.3 & 0.5 & 400 & 67 \\
\hline Lake & 12.3 & 7.7 & 19.3 & 7.6 & 8.3 & 12.7 & 3 & 67 \\
\hline Lee & 28.2 & 13.6 & 30.4 & 20.2 & 11.4 & 38.3 & 36 & 90 \\
\hline Leon & 0.4 & 0.0 & 0.3 & 0.2 & 2.2 & 0.5 & 25 & 150 \\
\hline Levy & 42.1 & 0.0 & 0.1 & 1.1 & 4.5 & 3.0 & -93 & 173 \\
\hline Liberty & 0.1 & 0.5 & 0.0 & 0.0 & 0.0 & 0.0 & -100 & 0 \\
\hline Madison & 0.5 & 0.1 & 0.2 & 1.1 & 0.0 & 0.3 & -40 & -73 \\
\hline Manatee & 5.1 & 9.6 & 46.3 & 32.6 & 34.3 & 45.3 & 788 & 39 \\
\hline Marion & 1.6 & 1.2 & 0.9 & 1.1 & 3.9 & 1.9 & 19 & 73 \\
\hline Martin & 44.5 & 74.9 & 76.8 & 147.3 & 142.9 & 153.9 & 246 & 4 \\
\hline Monroe & 0.0 & 0.0 & 0.0 & 0.0 & 0.0 & 0.0 & $\mathrm{~N} / \mathrm{A}$ & $\mathrm{N} / \mathrm{A}$ \\
\hline Nassau & 0.1 & 0.0 & 0.0 & 0.9 & 0.9 & 0.6 & 500 & -33 \\
\hline Okaloosa & 0.2 & 0.0 & 0.5 & 0.2 & 0.0 & 0.0 & -100 & -100 \\
\hline Okeechobee & 6.5 & 6.5 & 18.1 & 14.9 & 5.3 & 5.4 & -17 & -64 \\
\hline Orange & 103.7 & 136.9 & 97.8 & 30.9 & 53.9 & 61.5 & -41 & 99 \\
\hline Osceola & 6.8 & 5.4 & 4.2 & 4.1 & 5.6 & 13.4 & 97 & 227 \\
\hline Palm Beach & 352.3 & 333.6 & 546.0 & 589.8 & 554.3 & 795.8 & 126 & 35 \\
\hline Pasco & 3.1 & 0.0 & 10.4 & 0.8 & 0.9 & 2.4 & -23 & 200 \\
\hline Pinellas & 0.2 & 0.0 & 0.0 & (). .5 & 0.6 & 1.4 & 600 & 180 \\
\hline Polk & 274.1 & 198.3 & 305.5 & 298.5 & 78.4 & 83.8 & -69 & -72 \\
\hline Putnam & 68.5 & 141.1 & 141.2 & 25.6 & 20.1 & 18.8 & -73 & -27 \\
\hline St. Johns & 1.8 & 1.1 & 0.1 & 0.5 & 1.1 & 1.4 & -22 & 180 \\
\hline St. Lucie & 139.3 & 159.5 & 319.3 & 183.1 & 163.6 & 170.7 & 23 & -7 \\
\hline Santa Rosa & 0.0 & 0.0 & 0.2 & 0.3 & 0.2 & 0.3 & 300 & 0 \\
\hline Sarasota & 1.0 & 2.6 & 2.3 & 1.8 & 0.5 & 3.2 & 220 & 78 \\
\hline Seminole & 6.0 & 2.7 & 0.0 & 0.4 & 2.1 & 1.8 & .70 & 350 \\
\hline Sumter & 0.2 & 0.0 & 0.2 & 0.3 & 0.4 & 0.5 & 150 & 67 \\
\hline Suwannee & 180.4 & 173.0 & 172.8 & 174.0 & 66.6 & 109.5 & -39 & -37 \\
\hline Taylor & 0.1 & 0.0 & 0.1 & 0.1 & 1.6 & 1.3 & 1.200 & 1,200 \\
\hline Union & 0.1 & 0.0 & 0.0 & 0.1 & 0.0 & 0.7 & 600 & 600 \\
\hline Volusia & 351.4 & 406.3 & 314.0 & 156.6 & 117.8 & 203.2 & -42 & 30 \\
\hline Wakulla & 1.0 & 0.4 & 0.5 & 0.1 & 0.0 & 0.0 & -100 & -100 \\
\hline Walton & 0.0 & 0.0 & 0.3 & 1.1 & 0.5 & 1.1 & 1,100 & 0 \\
\hline Washington & 0.3 & 0.0 & 0.1 & 0.1 & 0.1 & 0.1 & -67 & 0 \\
\hline Totals & $2,623.3$ & 2.825 .6 & $3,453.6$ & 2.937 .3 & 2.236 .6 & 2.867 .1 & 9 & -2 \\
\hline
\end{tabular}




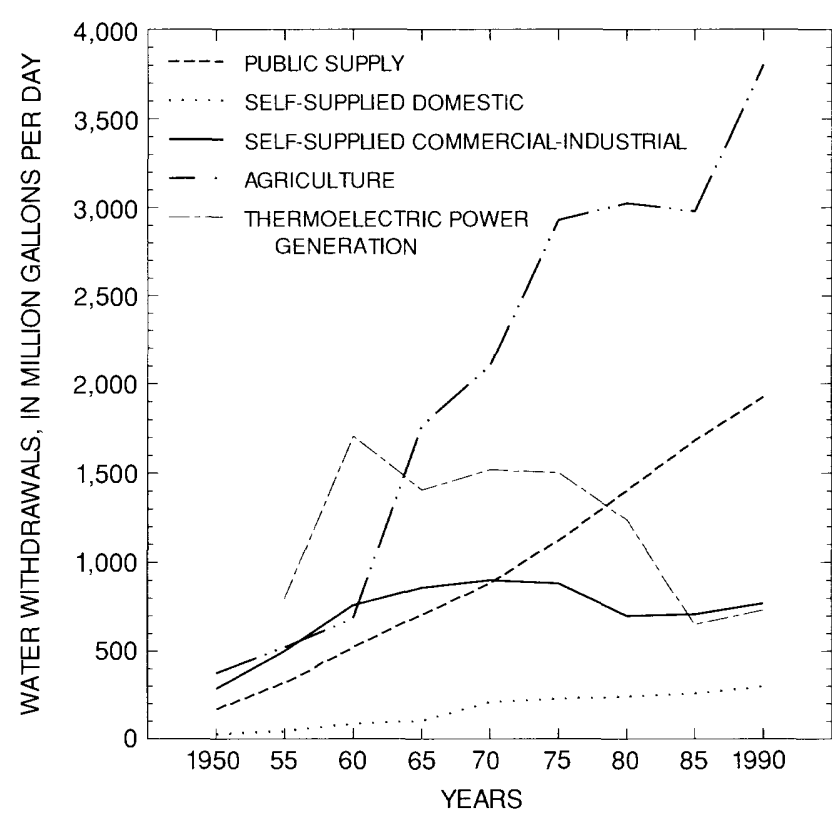

Figure 5. Freshwater withdrawn in Florida by category, 1950-90 (thermoelectric power generation value for 1950 was not available; modified from Marella, 1992b).

After 1980, livestock water use was included in the agriculture category as agricultural nonirrigation. Miscellaneous water withdrawals and uses were not included in this report. The miscellaneous category for 1980 and 1985 included water withdrawn for residential lawn irrigation, residential heat pumps and air conditioning units and water discharged through free-flowing wells. Because of the inconsistency in data from county to county for these uses, they were not included in the 1980 and 1985 Statewide totals. For 1990, information on residential lawn watering has been updated, and is included under agricultural irrigation as turf grass (other) in the 1990 Statewide report (Marella, 1992b).

\section{Public Supply}

The public-supply category refers to water supplied by a publicly- (for example, city, county, State, and others) or privately-owned water system for public distribution. According to the FDEP, any water system that serves more than 25 people or has 15 yearround service connections is considered a public supplier (Florida Department of Environmental Regulation, 1990, p. 6). Between 1950 and 1975, data for public supply usually were obtained directly from the utilities. After 1975, data for public supply basically were obtained from the FDEP (Drinking Water Section) monthly operating reports, and from the five water management districts consumptive water-use permit files. Public-supply water-use data for Florida were collected and compiled for 1950 , 1955, 1960, 1965, 1970, 1975, 1977, 1978, 1980, 1985, 1986, 1987, 1988, 1989, and 1990. Data for these years included the amount of water withdrawn by source (ground or surface) and the population served. Some public-supply water-use values have been adjusted or modified from previously published data. Nearly all of the modifications were a result of eliminating the previous double counting of water withdrawals for several utilities in some counties or adding utilities that were missed in the original inventory. Most of the double counting occurred when one public-supplier purchased water from another. The purchased water was sometimes counted as a withdrawal, and was inadvertently added to the county total, resulting in a double counting.

Water withdrawn for public supply in Florida increased from $170 \mathrm{Mgal} / \mathrm{d}$ in 1950 to $1,925 \mathrm{Mgal} / \mathrm{d}$ in 1990 (fig. 6 and table 6). Water withdrawn for publicsupply in 1980 and 1989 were greater than normal, primarily a result of dry conditions during these years. Ground-water withdrawals for public supply increased more than 1,110 percent from $140 \mathrm{Mgal} / \mathrm{d}$ in 1950 to 1,699 Mgal/d in 1990 (fig. 6 and table 6). Surfacewater withdrawals for public supply increased 653 percent from $30 \mathrm{Mgal} / \mathrm{d}$ in 1950 to $226 \mathrm{Mgal} / \mathrm{d}$ in 1990 (fig. 6 and table 6). All water withdrawn for public supply was from freshwater sources. However, since 1970, a small percentage of this water has not met the FDEP drinking water standards, and was considered nonpotable. This water, still considered fresh (contains less than $1,000 \mathrm{mg} / \mathrm{L}$ of dissolved solids), must be treated through desalination or diluted with fresher water to meet the FDEP drinking water standards for potable water (less than $500 \mathrm{mg} / \mathrm{L}$ of dissolved solids). The amount of nonpotable water treated or diluted in Florida for public supply use has increased from nearly $2 \mathrm{Mgal} / \mathrm{d}$ in 1970 to $48 \mathrm{Mgal} / \mathrm{d}$ in 1990 (table 6).

The population served by public supply in Florida increased from 1.66 million in 1950 to 11.23 million in 1990 (table 6). This increase generally mirrors the increase in the total population in Florida (fig. 1 and table 1), however, the percentage of the population served by public supply has increased significantly since 1950. 


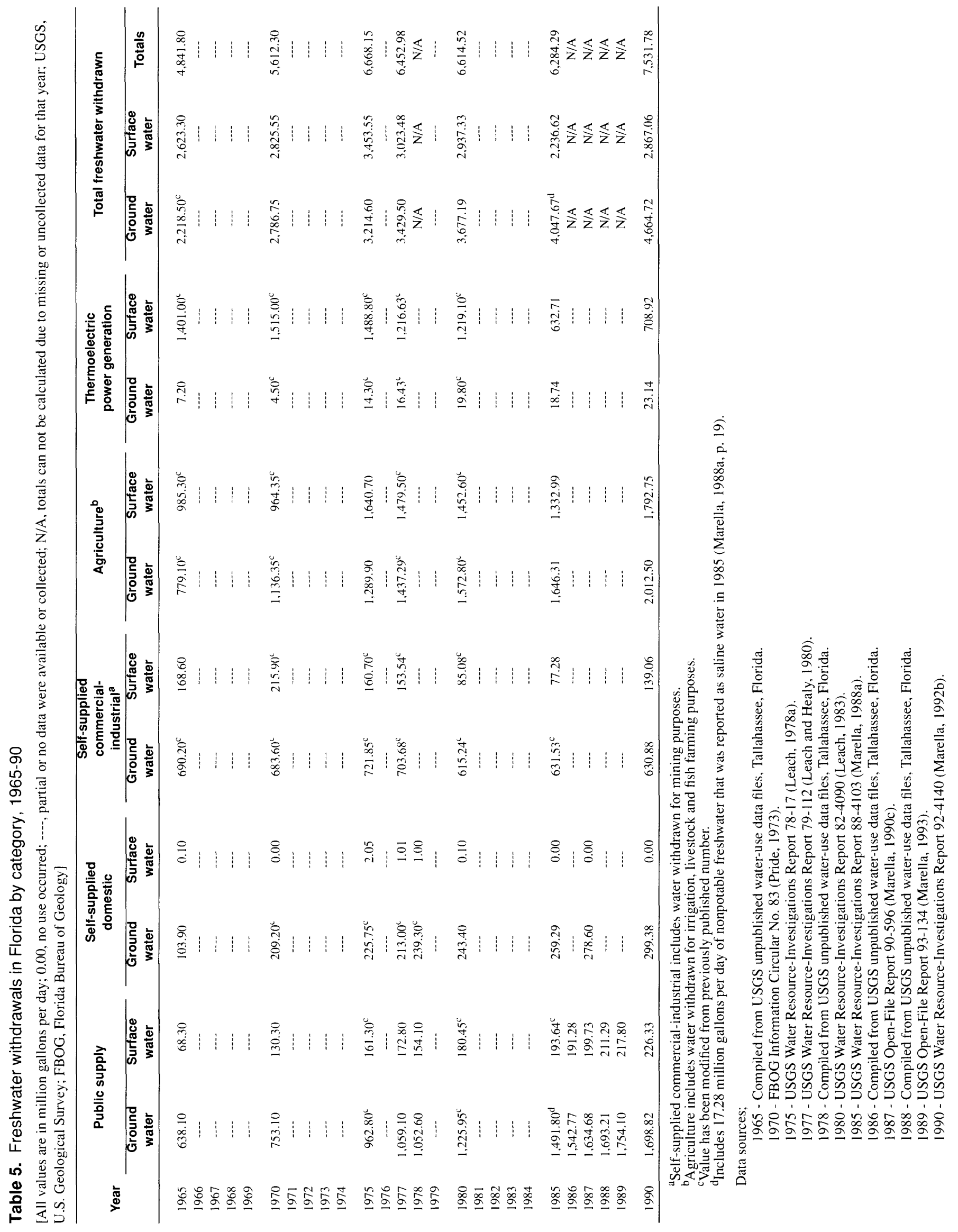




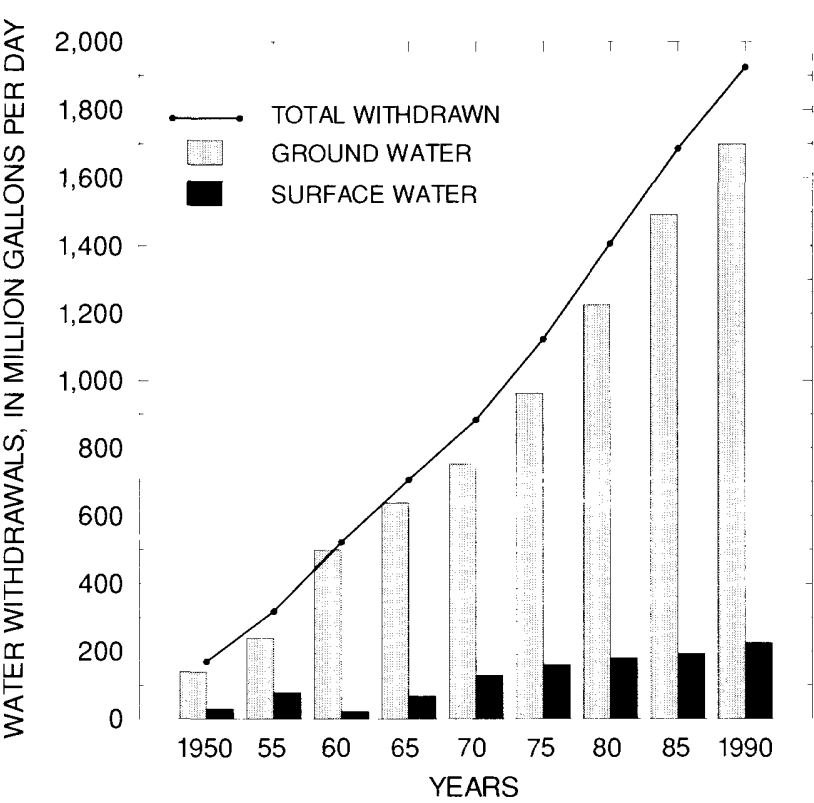

Figure 6. Public-supply freshwater withdrawn in Florida by source, 1950-90 (modified from Marella, 1992b).
In 1990, nearly 88 percent of Florida population relied on public-supply for their drinking water needs, compared to only 60 percent in 1950 . This trend can be attributed to the increase in the population of Florida, particularly in urban areas, and the expansion of publicsupply utility services (water and sewer) into unincorporated areas adjacent to the cities (Marella, 1992a).

Public suppliers provide water (deliveries) for a variety of users such as domestic (residential), commercial, industrial, and other uses. In addition to these uses, public-supply water is also reported for publicwater use, which includes firefighting, water lost to leakage, and system maintenance. Domestic water use, which includes indoor and outdoor residential uses, accounted for 65 percent of the use of publicsupply withdrawals in 1990 (Marella, 1992b, p. 15). The public-supply per capita use for Florida has increased from $102 \mathrm{gal} / \mathrm{d}$ in 1950 to $171 \mathrm{gal} / \mathrm{d}$ in 1990 (table 6). Public supply per capita use is the sum of the total public-supply water withdrawn divided by the total population served by public supply, and includes water delivered for commercial, industrial, publicwater use, and other uses as well as domestic uses.

Table 6. Public-supply population served and freshwater withdrawals in Florida by source, 1950-90

[All withdrawal values are in million gallons per day; modified from MacKichan (1951, 1957), MacKichan and Kammerer (1961), Murray (1968), Pride (1973), Leach (1978a. 1983), Leach and Healy (1980), Duerr and Trommer (1981b), and Marella (1987a, 1988a, 1990c, 1993); N/A, data not available]

\begin{tabular}{|c|c|c|c|c|c|c|c|}
\hline \multirow[b]{2}{*}{ Year } & \multirow{2}{*}{$\begin{array}{l}\text { Population } \\
\text { served by } \\
\text { public supply } \\
\text { (in millions) }\end{array}$} & \multicolumn{3}{|c|}{ Water withdrawn by source } & \multirow{2}{*}{$\begin{array}{c}\text { Treated } \\
\text { nonpotable } \\
\text { water }^{a}\end{array}$} & \multirow{2}{*}{$\begin{array}{l}\text { Inter-county } \\
\text { Water } \\
\text { transfers }^{b}\end{array}$} & \multirow{2}{*}{$\begin{array}{c}\text { Per capita, } \\
\text { in gallons } \\
\text { per day }\end{array}$} \\
\hline & & $\begin{array}{l}\text { Ground } \\
\text { water }\end{array}$ & $\begin{array}{c}\text { Surface } \\
\text { water }\end{array}$ & Totals & & & \\
\hline 1950 & 1.66 & 140.0 & 30.0 & 170.0 & $\mathrm{~N} / \mathrm{A}$ & N/A & 102 \\
\hline 1955 & 2.30 & 240.0 & 79.0 & 319.0 & N/A & N/A & 139 \\
\hline 1960 & 3.37 & 500.0 & 23.0 & 523.0 & N/A & N/A & 155 \\
\hline 1965 & 4.81 & 638.1 & 68.3 & 706.4 & $\mathrm{~N} / \mathrm{A}$ & $\mathrm{N} / \mathrm{A}$ & 147 \\
\hline 1970 & 5.42 & 753.1 & 130.3 & 883.4 & 1.6 & 48.6 & 163 \\
\hline 1975 & 6.81 & $962.8^{\mathrm{c}}$ & $161.3^{\mathrm{c}}$ & $1,124.1$ & 1.7 & 60.3 & 165 \\
\hline 1977 & 6.99 & $1,059.1$ & 172.8 & $1,231.9$ & 1.2 & 68.0 & 176 \\
\hline 1978 & 7.05 & $1,052.6$ & 154.1 & $1,206.7$ & 2.0 & 88.4 & 171 \\
\hline 1980 & 7.79 & $1,225.9^{c}$ & $180.5^{\mathrm{c}}$ & $1,406.4$ & 2.5 & 99.7 & 181 \\
\hline 1985 & 9.74 & $1,491.8^{\mathrm{d}}$ & $193.6^{\mathrm{c}}$ & $1,685.4$ & $17.3^{d}$ & 161.9 & 173 \\
\hline 1986 & 10.11 & $1,542.8$ & 191.3 & $1,734.1$ & N/A & 127.7 & 172 \\
\hline 1987 & 10.35 & $1,634.7$ & $199.7^{c}$ & $1,834.4$ & 37.4 & 127.9 & 177 \\
\hline 1988 & 10.66 & $1,693.2$ & 211.3 & $1,904.5$ & N/A & 130.5 & 179 \\
\hline 1989 & 10.93 & $1,754.1$ & 217.8 & $1,971.9$ & 42.4 & 149.5 & 180 \\
\hline 1990 & 11.23 & $1,698.8$ & 226.3 & $1,925.1$ & 47.9 & 138.7 & 171 \\
\hline
\end{tabular}

${ }^{a}$ Nonpotable water treated to meet the Florida Department of Environmental Protection secondary drinking standards. This water is treated through desalination or diluted with fresh water to meet the required standards and is still considered freshwater.

${ }^{b}$ Water transfers refer to the artificial conveyance of water from one area to another. Inter-county transfers refer to the amount of water moved from one county to another for public supply uses.

${ }^{c}$ Value has been modified from previously published number.

${ }^{\mathrm{d}}$ Treated nonpotable water withdrawal value for 1985 was reported as saline water in Marella, 1988a. 
Public-supply water use increased in all 67 counties in Florida between 1965 and 1990. Hernando County accounted for the largest percent increase between 1965 and 1990, and between 1980 and 1990 (2,900 percent and 1,264 percent, respectively) due to growth generated from the nearby Tampa area (table 7). Between 1980 and 1990, public supply water use increased in all counties except Gilchrist and Taylor Counties, in which water usage remained the same or decreased. Public supply water-use in Dade County exceeded $200 \mathrm{Mgal} / \mathrm{d}$ in 1970 and 1975, and 300 $\mathrm{Mgal} / \mathrm{d}$ in 1980, 1985, and 1990 (table 7). In Dade County, the Miami-Dade Water and Sewer Authority, the single largest water supplier in the State, withdrew more than $153 \mathrm{Mgal} / \mathrm{d}$ in 1970 (Healy, 1972, p. 171) and $296 \mathrm{Mgal} / \mathrm{d}$ in 1990 (Marella, 1993, p. 26). Dade County also supplies water (exports) to neighboring Monroe County for public-supply use throughout the Florida Keys (Meyer, 1974). Other counties that are involved in water transfers include Brevard, Charlotte, De Soto, Hillsborough, Lee, Manatee, Orange, Pasco, Pinellas, and Sarasota. In 1990, nearly $139 \mathrm{Mgal} / \mathrm{d}$ of public-supplied water was withdrawn from one county for use in another county, compared to $49 \mathrm{Mgal} / \mathrm{d}$ in 1970 (table 6).

\section{Self-Supplied Domestic}

Self-supplied domestic use is water provided by individual domestic wells or by small utility systems serving less than 400 people not inventoried for public supply. In 1990, an estimated 1.71 million people in Florida were self-supplied or obtained water from small utility systems, compared to 1.11 million in 1950 (table 8). Self-supplied population figures are derived by subtracting the number of residents served by public-supplied systems from the total populations for each county. Self-supplied domestic water-use data for Florida were collected and compiled for 1950, 1955, 1960, 1965, 1970, 1975, 1977, 1978, 1980, 1985, 1987, and 1990. Data for these years included estimates of the amount of water withdrawn and the self-supplied population. Early water-use estimates for this category (1950 through 1980) included domestic uses along with livestock uses. More recent values include only domestic water use estimates, as livestock water use was moved under the agriculture category. Water-use figures for those years (1950 through 1980) have been modified to include only the domestic values.
Water withdrawn for self-supplied use in Florida has increased from $27 \mathrm{Mgal} / \mathrm{d}$ in 1950 to $299 \mathrm{Mgal} / \mathrm{d}$ in 1990 (fig. 7 and table 8). Withdrawals are calculated by multiplying the public-supply per capita for each county (in gallons per day) by the self-supplied population for each county. The public-supply per capita value for each county was derived by taking the public supply net water-use in the county and dividing it by the population served by the public supply for the county. It is assumed that water withdrawn for self-supplied domestic use in Florida is derived solely from ground water, primarily because this source can provide the quantity and quality of water needed for drinking purposes. During the 1970 's, a small percentage of domestic water withdrawals were estimated to be from surface water sources, however, by 1990, surface water would not likely meet the State's drinking-water standards without proper treatment in most areas of Florida.

Self-supplied water use increased in 60 counties in Florida between 1965 and 1990 (table 9). Pinellas County accounted for the largest percent increase between 1965 and 1990 (1,900 percent) (table 9). Most of the increase in water use during this period throughout Florida was a result of a change in tabulation methods. From 1950 to 1970 , an estimated statewide per capita figure of $120 \mathrm{gal} / \mathrm{d}$ was used. After 1970, individual county public-supply per capita rates were used. This change was made to help make each county better reflect its public-supply water use tendencies, and to help account for the many small self-supplied commercial and industrial systems that use water for domestic purposes but are not included under the commercialindustrial self-supplied category because they are too small to be inventoried. Between 1980 and 1990, selfsupplied domestic water use increased in only 43 counties (table 9). This is primarily a result of public-supply utility expansion into areas that were once self-supplied. Many of the counties that experienced self-supplied water-use decreases between 1980 and 1990 also experienced large increases in publicsupply water use between 1980 and 1990 (table 7). This is evident in several highly-developed counties such as Duval (Jacksonville), Hillsborough (Tampa), and Orange (Orlando). Only Monroe County had no self-supplied domestic population or use, primarily because the quality of the ground- and surface-water is nonpotable. However, a small unrecorded amount of ground-water is used for nonpotable domestic needs throughout the Florida Keys in Monroe County (McKenzie, 1990, p. 1). 
Table 7. Public-supply freshwater use and percent change in Florida by county, 1965-90

IModified from Pride (1973), Leach (1978a, 1983), and Marella (1988a, 1992b); water use values represent the net total use for the county, plus or minus transfers; values may not add to totals because of independent rounding or revisions in data]

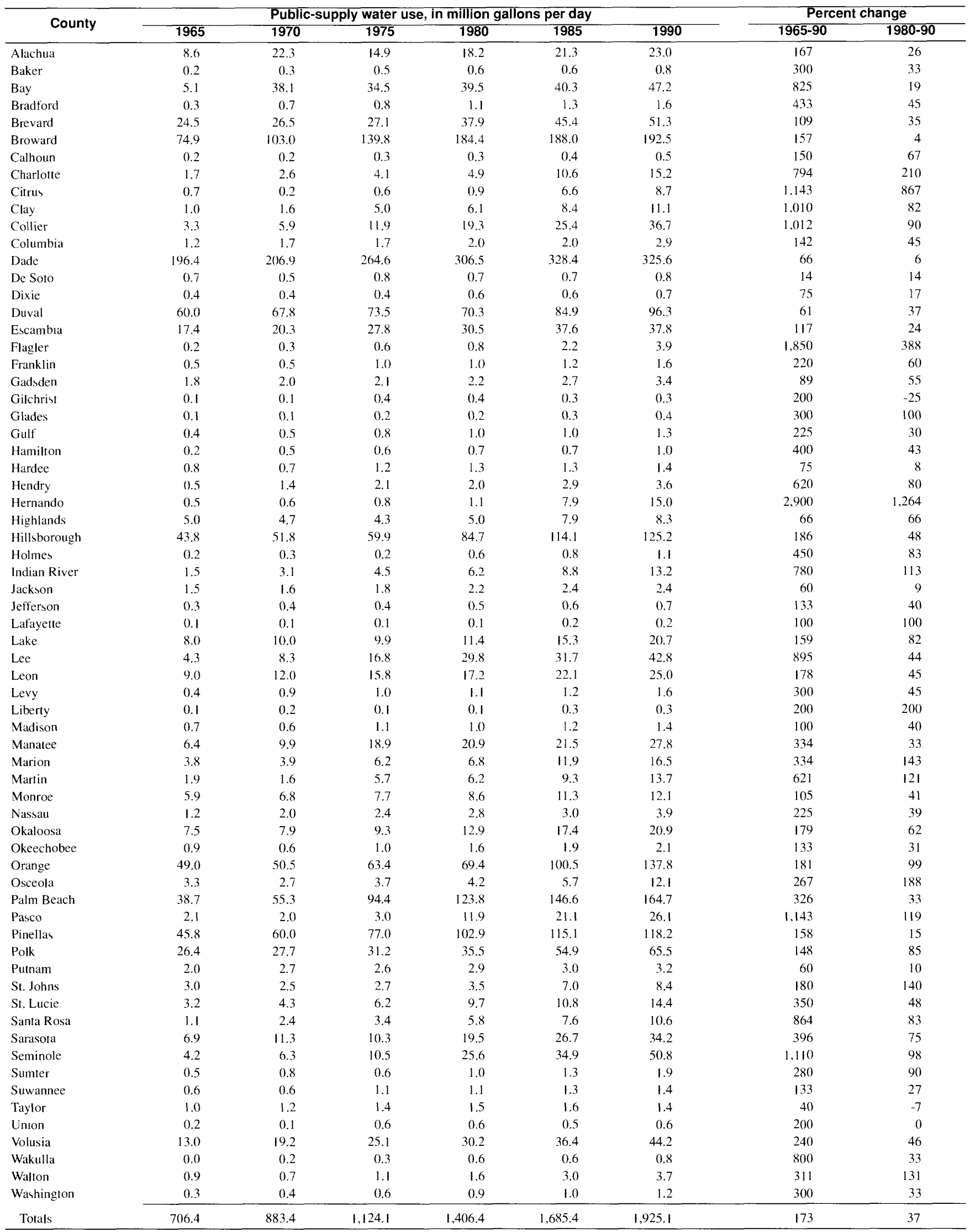


Table 8. Self-supplied domestic population and freshwater withdrawals in Florida by source, 1950-90

[All withdrawal values are in million gallons per day; modified from MacKichan (1951, 1957), MacKichan and Kammerer (1961), Murray (1968), Pride (1973), Leach (1978a, 1983), Leach and Healy, (1980), and Marella (1988a, 1990c, 1992b)]

\begin{tabular}{|c|c|c|c|c|}
\hline \multirow[b]{2}{*}{ Year } & \multirow{2}{*}{$\begin{array}{l}\text { Self-supplied } \\
\text { domestic } \\
\text { population } \\
\text { (in millions) }\end{array}$} & \multicolumn{3}{|c|}{ Water withdrawn by source ${ }^{a}$} \\
\hline & & $\begin{array}{l}\text { Ground } \\
\text { water }\end{array}$ & $\begin{array}{c}\text { Surface } \\
\text { water }\end{array}$ & Totals \\
\hline 1950 & 1.11 & 27.0 & 0.0 & 27.0 \\
\hline 1955 & 1.30 & 45.0 & 0.0 & 45.0 \\
\hline 1960 & 1.58 & 86.0 & 0.0 & 86.0 \\
\hline 1965 & 1.10 & 103.9 & 0.1 & 104.0 \\
\hline 1970 & 1.37 & $209.2^{b}$ & 0.0 & 209.2 \\
\hline 1975 & 1.87 & $225.8^{b}$ & 2.1 & 227.9 \\
\hline 1977 & 1.73 & $213.0^{\mathrm{b}}$ & 1.0 & 214.0 \\
\hline $1978^{c}$ & 1.92 & 239.3 & 1.0 & 240.3 \\
\hline 1980 & 1.95 & $243.4^{b}$ & 0.1 & 243.5 \\
\hline 1985 & 1.59 & 259.3 & 0.0 & 259.3 \\
\hline $1987^{\circ}$ & 1.65 & 278.6 & 0.0 & 278.6 \\
\hline 1990 & 1.71 & 299.4 & 0.0 & 299.4 \\
\hline
\end{tabular}

ancludes water withdrawn for self-supplied domestic use only, and does not include livestock water uses as reported under rural water-use between 1950 and 1980 .

${ }^{b}$ Value has been modified from previously published number.

${ }^{c}$ Estimated from unpublished data.

\section{Self-Supplied Commercial-Industrial}

Self-supplied commercial use includes water withdrawn at the following facilities: government, military, schools, prisons, hospitals, recreational, and nonmanufacturing establishments such as hotels or motels, laundry facilities, and restaurants. Selfsupplied industrial use includes water withdrawn at mining, processing, and manufacturing facilities. Prior to 1975 , water-use data for this category were usually obtained by contacting the facility directly. Beginning in 1975, water-use data for this category were collected by each water management district's consumptive water-use permit program (compliance files), or from the monthly operating reports submitted to the FDEP. Not all facilities are required to submit

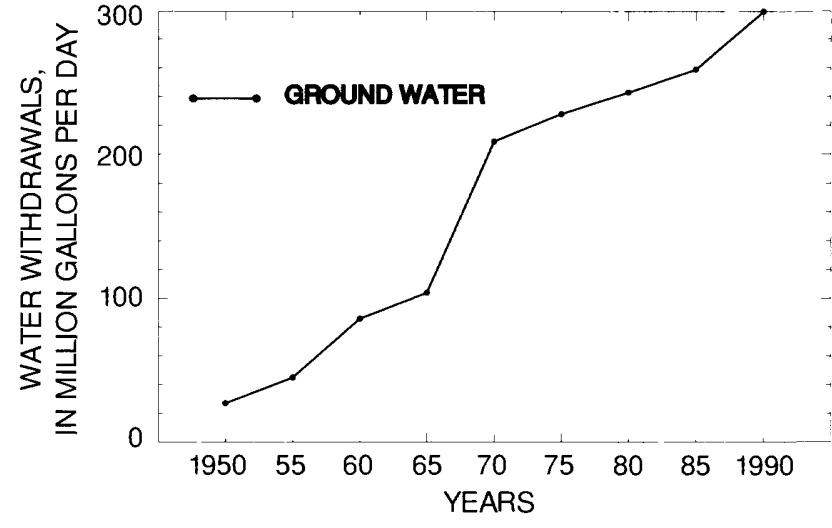

Figure 7. Self-supplied domestic ground water withdrawn in Florida, 1950-90 (modified from Marella, 1992b).

pumpage to the water management districts or FDEP, therefore data for these facilities were also obtained by direct contact by the water management districts or USGS. Self-supplied commercial-industrial water-use data for Florida were collected and compiled for 1950, 1955, 1960, 1965, 1970, 1975, 1977, 1980, 1985, and 1990. Data for these years include the amount of water withdrawn by source (ground or surface and fresh or saline) but do not include the amount of water recirculated within these facilities.

Some commercial-industrial water-use values have been adjusted or modified from previously published data. Most modifications were a result of adding missing systems, reclassifying cooling water used for power generation, or adjusting water withdrawals for heat exchange that allows water to be returned entirely to the source through a closed system. Specific changes for commercial-industrial use occurred in several counties where ground water was withdrawn for heating and cooling (heat exchange units). These large heating and air conditioning units are common in Florida because abundant ground water is available throughout the year. These units are used in large office buildings or complexes, hotels, and many commercial or industrial facilities. In these systems well water is pumped through a chiller, where the exchange of the water temperature occur with the freon gas in the unit. Usually, the water remains in a closed loop system and is immediately pumped back into the aquifer. 
Table 9. Self-supplied domestic freshwater use and percent change in Florida by county, 1965-90

[Modified from Pride (1973), Leach (1978a, 1983), and Marella (1988a, 1992b); does not include livestock water use as reported for 1975 and 1980; values may not add to totals because of independent rounding or revisions in data]

\begin{tabular}{|c|c|c|c|c|c|c|c|c|}
\hline County & \multicolumn{6}{|c|}{ Self-supplied domestic water use, in million gallons per day } & \multicolumn{2}{|c|}{ Percent change } \\
\hline Baker & 0.3 & 0.8 & 0.8 & 1.6 & 1.9 & 2.8 & 833 & 75 \\
\hline Bay & 1.8 & 4.4 & 0.9 & 1.6 & 5.6 & 5.5 & 206 & 244 \\
\hline Brevard & 4.0 & 3.4 & 2.7 & 4.1 & 5.6 & 5.1 & 28 & 24 \\
\hline Broward & 10.2 & 8.4 & 8.2 & 5.9 & 3.0 & 9.6 & -6 & 63 \\
\hline Calhoun & 0.8 & 0.5 & 0.5 & 0.7 & 0.9 & 1.2 & 50 & 71 \\
\hline Clay & 0.6 & 2.3 & 1.8 & 7.4 & 2.7 & 4.5 & 650 & -39 \\
\hline Collier & 1.3 & 1.0) & 0.4 & 2.3 & 3.3 & 8.9 & 585 & 287 \\
\hline Columbia & 0.6 & 1.0) & 1.3 & 3.8 & 4.9 & 5.2 & 767 & 37 \\
\hline Dade & 9.6 & 9.1 & 9.5 & 18.4 & 13.3 & 10.8 & 13 & -41 \\
\hline De Soto & 0.4 & 0.9 & 1.1 & 1.2 & 1.7 & 1.9 & 375 & 58 \\
\hline Dixie & 0.1 & 0.4 & 0.3 & 0.4 & 0.7 & 0.9 & 800 & 125 \\
\hline Duval & 17.4 & 36.2 & 32.9 & 20.3 & 15.5 & 8.4 & -52 & -59 \\
\hline Gilchrist & 0.1 & 0.3 & 0.4 & 0.5 & 1.2 & 1.3 & 1.200 & 160 \\
\hline Glades & 0.1 & 0.3 & 0.4 & 0.7 & 0.6 & 0.6 & 500 & -14 \\
\hline Gulf & 0.2 & 0.5 & 0.4 & 0.5 & 0.7 & 0.3 & 50 & -40 \\
\hline Hamilton & 0.3 & 0.4 & 0.3 & 0.6 & 1.0 & 1.1 & 267 & 83 \\
\hline Hardee & 0.3 & 1.0 & 1.2 & 1.2 & 1.8 & 1.9 & 533 & 58 \\
\hline Hendry & 0.7 & 0.7 & 0.7 & 0.8 & 0.8 & 2.0 & 186 & 150 \\
\hline Hernando & 0.4 & 1.4 & 2.4 & 3.9 & 1.0 & 1.8 & 350 & -54 \\
\hline Highlands & 0.4 & 0.7 & 1.8 & 3.4 & 2.8 & 3.4 & 750 & 0 \\
\hline Hillsborough & 6.5 & 14.4 & 21.3 & 5.9 & 6.1 & 2.7 & -58 & -54 \\
\hline Holmes & 0.5 & 0.9 & 0.9 & 1.3 & 2.0 & 2.3 & 360 & 77 \\
\hline Indian River & 1.0 & 1.8 & 2.8 & 4.) & 7.3 & 8.9 & 790 & 123 \\
\hline Jackson & 1.1 & 2.3 & 2.5 & 2.6 & 4.1 & 3.9 & 255 & 50 \\
\hline Jefferson & 0.3 & 0.7 & 0.4 & 0.9 & 1.3 & 1.0 & 233 & 11 \\
\hline Lafayette & 0.1 & 0.2 & 0.2 & 0.3 & 0.6 & 0.7 & 600 & 133 \\
\hline Monroe & 0.0 & 0.0 & 0.0 & 0.0 & 0.0 & 0.0 & 0 & 0 \\
\hline Nassau & 0.5 & 1.4 & 1.8 & 4.6 & 4.0 & 3.8 & 660 & -17 \\
\hline Okatoosa & 2.0 & 3.0 & 2.2 & 3.8 & 2.2 & 2.8 & 40 & -26 \\
\hline Okeechobee & 0.3 & 0.2 & 0.9 & 1.6 & 1.6 & 1.6 & 433 & 0 \\
\hline Orange & 4.0 & 7.6 & 8.6 & 10.7 & 6.1 & 5.0 & 25 & -53 \\
\hline Osceola & 0.5 & 2.0 & 1.8 & 3.7 & 4.8 & 4.3 & 760 & 16 \\
\hline Palm Beach & 6.9 & 10.7 & 12.9 & 10.7 & 5.6 & 21.3 & 209 & 99 \\
\hline Pasco & 0.8 & 6.2 & 10.4 & 5.7 & 8.3 & 10.3 & 1,188 & 81 \\
\hline Pinellas & 0.1 & 13.1 & 6.5 & 2.7 & 3.7 & 2.0 & 1,900 & -26 \\
\hline Polk & 2.1 & 7.0 & 9.3 & 13.8 & 18.2 & 23.3 & 1.010 & 69 \\
\hline Putnam & 0.9 & 2.7 & 2.9 & 4.8 & 6.4 & 5.9 & 556 & 23 \\
\hline St. Johns & 0.6 & 1.7 & 2.4 & 2.4 & 2.1 & 2.2 & 267 & -8 \\
\hline St. Lucie & 1.7 & 2.0 & 4.) & 3.2 & 6.4 & 11.5 & 576 & 259 \\
\hline Santa Rosa & 1.5 & 2.8 & 1.1 & 0.7 & 0.8 & 0.6 & -60 & -14 \\
\hline Sarasota & 0.9 & 1.9 & 7.3 & 1.1 & 3.1 & 3.9 & 333 & 255 \\
\hline Seminole & 1.8 & 2.7 & 8.1 & 11.7 & 3.6 & 3.1 & 72 & -74 \\
\hline Sumter & 0.8 & 1.2 & 1.4 & 1.9 & 2.4 & 3.0 & 275 & 58 \\
\hline Suwannee & 0.5 & 0.9 & 1.0 & 1.5 & 2.5 & 3.0 & 500 & 100 \\
\hline Taylor & 0.2 & 0.4 & 0.4 & 0.8 & 1.1 & 1.0 & 400 & 25 \\
\hline Union & 0.2 & 0.8 & 0.9 & 0.5 & 0.9 & 2.4 & 1,100 & 380 \\
\hline Volusia & 1.7 & 3.7 & 6.5 & 4.5 & 5.3 & 6.8 & 300 & 51 \\
\hline Wakulla & 0.3 & 0.5 & 0.4 & 0.7 & 0.7 & 0.7 & 133 & 0 \\
\hline Walton & 0.5 & 0.8 & 0.7 & 0.9 & 0.5 & 0.4 & -20 & -56 \\
\hline Washington & 0.4 & 0.9 & 0.8 & 0.9 & 1.2 & 1.7 & 325 & 89 \\
\hline Totals & 104.0 & 209.2 & 227.9 & 243.5 & 259.3 & 299.4 & 188 & 23 \\
\hline
\end{tabular}


Withdrawals for these units were eliminated from the county totals as long as the system remained closed and the water makes no contact with the air or light of day. Additionally, withdrawal data only existed for a selected number of systems, and the data for these systems was never collected or tabulated with any consistency between 1970 and 1990. Other significant changes were made for mining facilities, as water withdrawn for dewatering purposes often was categorized as surface water in some years and as ground water in other years. This was particularly common when the dewatering occurred directly from the mine pit. The water in the mine pit was mostly ground water, as the water table of the aquifer was exposed. For some years and for some agencies, this water was considered ground water and for others it was considered surface water. For consistency, modifications were made to categorize all dewatering uses as ground water. Furthermore, water used at mining facilities often was not differentiated between processing or dewatering because of the reporting practices of the facility. In more recent years many facilities began reporting water used for processing only, and excluding the amount of water withdrawn for dewatering purposes. Another significant change that affected self-supplied commercial-industrial water-use values was a change in reporting procedures for water withdrawn for cooling purposes. During the 1950's and 1960 's, water withdrawn for power generation within a industrial facility often was reported under the thermoelectric category. More recently (1965 to 1990), all withdrawals for industrial purposes, regardless of use (power generation or processing), were reported under the self-supplied commercial-industrial category.

Freshwater withdrawn for self-supplied commercial-industrial uses in Florida has increased from $286 \mathrm{Mgal} / \mathrm{d}$ in 1950 to $770 \mathrm{Mgal} / \mathrm{d}$ in 1990 (fig. 8 and table 10). However, water withdrawn for self-supplied commercial-industrial use was greatest at nearly $900 \mathrm{Mgal} / \mathrm{d}$ in 1970 . An additional $56 \mathrm{Mgal} / \mathrm{d}$ of saline surface-water was withdrawn for self-supplied commercial-industrial purposes in 1990. Most of the saline water is used for once-through cooling at several of the larger industrial facilities along the coast. Fresh ground-water withdrawals for self-supplied commercial-industrial use increased 125 percent from $280 \mathrm{Mgal} / \mathrm{d}$ in 1950 to $631 \mathrm{Mgal} / \mathrm{d}$ in 1990 (table 10). Fresh surface-water withdrawals for self-supplied commercial-industrial increased nearly 2,220 percent from $6 \mathrm{Mgal} / \mathrm{d}$ in 1950 to $139 \mathrm{Mgal} / \mathrm{d}$ in 1990 (table 10).

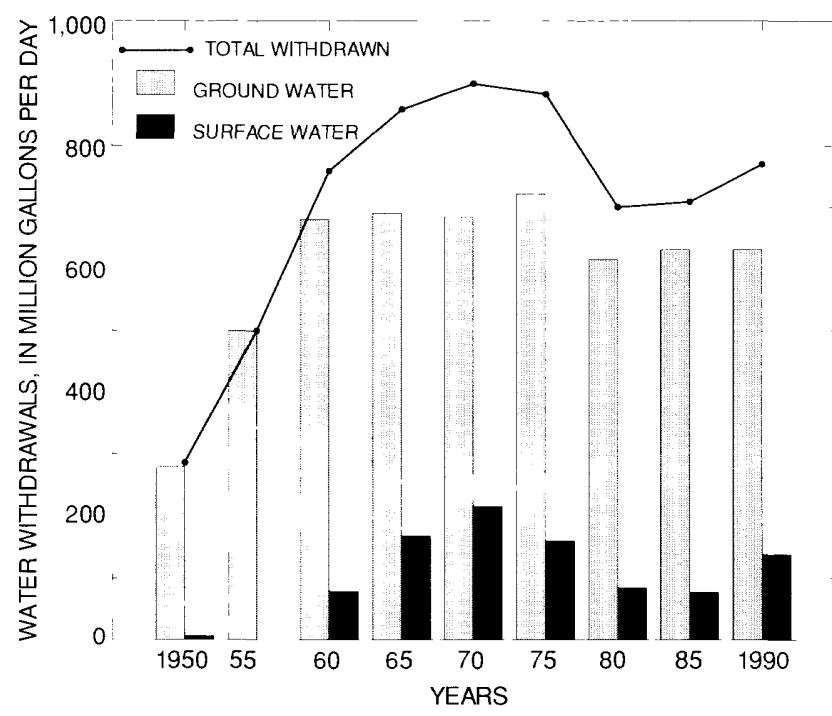

Figure 8. Self-supplied commercial-industrial freshwater withdrawn in Florida by source, 1950-90 (modified from Marella, 1992b).

Table 10. Self-supplied commercial-industrial water withdrawals in Florida by source, 1950-90

[All values are in million gallons per day; includes water withdrawn for self-supplied commercial, industrial, and mining uses; modified from MacKichan (1951, 1957), MacKichan and Kammerer (1961), Murray (1968), Pride (1973), Leach (1978a, 1983), Leach and Healy (1980), and Marella (1988a, 1992b) ]

\begin{tabular}{|c|c|c|c|c|}
\hline \multirow[b]{2}{*}{ Year } & \multicolumn{3}{|c|}{ Freshwater withdrawn by source } & \multirow{2}{*}{$\begin{array}{l}\text { Saline water } \\
\text { withdrawn }^{\text {a }}\end{array}$} \\
\hline & $\begin{array}{l}\text { Ground } \\
\text { water }\end{array}$ & $\begin{array}{c}\text { Surface } \\
\text { water }\end{array}$ & Totals & \\
\hline 1950 & 280.0 & 6.0 & 286.0 & 0.0 \\
\hline 1955 & 500.0 & 0.0 & 500.0 & 15.0 \\
\hline 1960 & 680.0 & 79.0 & 759.0 & 260.0 \\
\hline 1965 & $690.2^{b}$ & 168.6 & 858.8 & 61.2 \\
\hline 1970 & $683.6^{\mathrm{b}}$ & $215.9^{b}$ & 899.5 & 132.5 \\
\hline 1975 & $721.9^{b}$ & $160.7^{b}$ & 882.6 & 63.0 \\
\hline 1977 & $703.7^{b}$ & $153.5^{\mathrm{b}}$ & 857.2 & 73.5 \\
\hline 1980 & $615.4^{b}$ & $85.1^{\mathrm{b}}$ & 700.5 & 57.5 \\
\hline 1985 & $631.5^{b}$ & 77.3 & 708.8 & 26.9 \\
\hline 1990 & 630.9 & 139.1 & 770.0 & 56.4 \\
\hline
\end{tabular}

ancludes both ground and surface water withdrawals.

${ }^{b}$ Value has been modified from previously published number.

Total freshwater withdrawn for self-supplied commercial-industrial use in Florida decreased between 1970 (900 Mgal/d), and 1980 (700 Mgal/d), but increased between 1980 and 1990 (770 Mgal/d) (fig. 8). 
However, for the same period (1970 and 1990), deliveries to commercial and industrial users from publicsupply systems increased nearly $300 \mathrm{Mgal} / \mathrm{d}$ (Marella, 1992b, p. 22). Because of the increasing cost of treating water and discharging wastewater, many industries obtain water from a public supplier and (or) reuse (recycle) as much water as possible. Increases in water conservation practices and restrictions on withdrawals and discharges in Florida over the last 20 years have also had effects on the amount of water withdrawn for this category. Surface-water values for 1955 are believed to include values for thermoelectric power generation, as these two categories (selfsupplied commercial-industrial and thermoelectric power generation) were combined in the published tables for that year (MacKichan, 1957).

Mining accounted for the largest single use of freshwater within the self-supplied commercialindustrial category during 1990 (41 percent), followed by the pulp and paper industry ( 25 percent), chemical manufacturing (14 percent), food production and manufacturing ( 12 percent), and miscellaneous manufacturing (2 percent) (Marella, 1992b, p. 22).

Commercial water uses accounted for the remaining 6 percent in Florida during 1990. An additional $282 \mathrm{Mgal} / \mathrm{d}$ and $183 \mathrm{Mgal} / \mathrm{d}$ were delivered by publicsupply systems to commercial and industrial water users, respectively, during 1990 (Marella, 1992b, p. 19).

Self-supplied commercial-industrial water use increased in only 40 counties between 1965 and 1990. Freshwater withdrawals for this category occurred in 45 counties in 1965 compared to 62 in 1990 (table 11). Although Polk County had the largest county use of freshwater for 1965 through 1990, its use decreased 31 percent during this time. The majority of water withdrawn in Polk County during this period was for phosphate mining and associated chemical manufacturing. Reduction of water usage in the phosphate industry has occurred over the past 25 years mainly because of the extensive recirculation of water used in mining operations. Recent estimates indicate that an average of 90 percent of the water used in phosphate mining is recirculated (Campbell, 1986, p. 36). Overall, mining water use has decreased from $347 \mathrm{Mgal} / \mathrm{d}$ in 1970 (Pride, 1973, p. 18) to $315 \mathrm{Mgal} / \mathrm{d}$ in 1990 (Marella, 1992b, p. 22). However, water used for limerock and sand mining increased from $28 \mathrm{Mgal} / \mathrm{d}$ to $188 \mathrm{Mgal} / \mathrm{d}$ during this period.
Limerock and sand primarily are used for construction purposes such as road base material, concrete and asphalt aggregate, and cement manufacturing. Limerock and sand are predominately mined in Broward, Collier, Dade, Hernando, Lake, Lee, Marion, Monroe, Okeechobee, Palm Beach, and Suwannee Counties (Campbell, 1986, p. 51, Schmidt and others, 1979, p. 14-15). Reported water withdrawal values for counties involved in mining may often change significantly from year to year, as a result of a closure of a mine or a pit within the mine or the opening of a new mine or pit. Pulp and paper manufacturing water use also decreased from $237 \mathrm{Mgal} / \mathrm{d}$ in 1970 (Pride, 1973, p. 18) to $189 \mathrm{Mgal} / \mathrm{d}$ in 1990 (Marella, 1992b, p. 22). Pulp and paper manufacturing in Florida primarily are located in Duval, Escambia, Gulf, Nassau, Putnam, and Taylor Counties. Recirculation of process water throughout the pulp and paper facilities also has increased between 1970 and 1990.

\section{Agriculture (Irrigation and Nonirrigation)}

Agriculture water use includes withdrawals for irrigation and nonirrigation. Irrigation withdrawals include supplementing rainfall for the growth of commercial crops, ornamentals, and grasses. Nonirrigation agricultural water use includes withdrawals for livestock needs, both drinking and washdown, and augmentation for fish farming. Since agriculture withdrawals generally have not been metered or measured throughout Florida between 1950 and 1990, water-use values are estimated for this category. Some metering has occurred in various places in the State, but this data was often too little or too infrequent to compile on a statewide level.

From 1950 to 1990 , water withdrawals for agriculture were calculated in two steps. First, the number of acres irrigated and method of irrigation for each of the crops inventoried within every county was determined. Prior to 1975, acreage data was obtained from the agricultural extension agent (University of Florida, Institute of Food and Agriculture Science) in each county, the U.S. Department of Agricultural, Agricultural Stabilization and Conservation Service (ASCS), the Soil Conservation Service (SCS) county offices, the U.S. Department of Commerce, Agricultural Census, and other State or local agencies. From 1975 to present, acreage data were obtained by each water 
Table 11. Self-supplied commercial-industrial freshwater use and percent change in Florida by county, 1965-90

[Modified from Pride (1973), Leach (1978a, 1983), and Marella (1988a, 1992b); N/A, not applicable; values may not add to totals because of independent rounding or revisions in data]

\begin{tabular}{|c|c|c|c|c|c|c|c|c|}
\hline \multirow{2}{*}{ County } & \multicolumn{6}{|c|}{ Self-supplied commercial-industrial water use, in million gallons per day } & \multicolumn{2}{|c|}{ Percent change } \\
\hline & 1965 & 1970 & 1975 & 1980 & 1985 & 1990 & $1965-90$ & $1980-90$ \\
\hline Alachua & 13.0 & 1.4 & 6.5 & 1.6 & 3.6 & 2.3 & -82 & 44 \\
\hline Baker & 0.2 & 0.0 & 0.3 & 0.3 & 0.7 & 0.9 & 350 & 200 \\
\hline Bay & 34.1 & 2.5 & 1.4 & 1.5 & 1.5 & 2.1 & -94 & 40 \\
\hline Bradford & 0.0 & 1.4 & 4.0 & 4.8 & 4.3 & 3.0 & 3,000 & -38 \\
\hline Brevard & 1.0 & 0.4 & 0.5 & 0.2 & 0.2 & 0.2 & -80 & 0 \\
\hline Broward & 3.0 & 3.0 & 3.5 & 1.2 & 1.4 & 1.6 & -47 & 33 \\
\hline Calhoun & 1.6 & 0.0 & 0.4 & 0.7 & 0.0 & 0.0 & -100 & -100 \\
\hline Charlotte & 0.0 & 0.1 & 0.1 & 0.0 & 0.0 & 4.3 & 4,300 & 4,300 \\
\hline Citrus & 0.2 & 0.2 & 1.3 & 0.6 & 0.9 & 2.1 & 950 & 250 \\
\hline Clay & 1.5 & 1.5 & 10.9 & 3.2 & 11.7 & 6.6 & 340 & 106 \\
\hline Collier & 0.5 & 0.5 & 0.0 & 2.3 & 4.7 & 4.3 & 760 & 87 \\
\hline Columbia & 0.0 & 0.0 & 0.1 & 0.3 & 0.1 & 0.1 & 100 & -67 \\
\hline Dade & 7.7 & 10.4 & 3.4 & 19.7 & 15.8 & 40.3 & 423 & 105 \\
\hline De Soto & 3.7 & 0.7 & 0.6 & 0.5 & 0.4 & 0.5 & -86 & 0 \\
\hline Dixie & 0.0 & 0.9 & 3.5 & 0.4 & 0.9 & 0.9 & 900 & 125 \\
\hline Duval & 48.0 & 60.9 & 48.8 & 40.5 & 38.1 & 33.9 & -29 & -16 \\
\hline Escambia & 80.8 & 90.7 & 76.5 & 55.0 & 51.4 & 51.7 & -36 & -6 \\
\hline Flagler & 0.0 & 0.0 & 0.0 & 0.0 & 0.1 & 0.3 & 300 & 300 \\
\hline Franklin & 0.2 & 0.0 & 0.0 & 0.0 & 0.0 & 0.0 & -100 & 0 \\
\hline Gadsden & 2.3 & 2.1 & 2.0 & 1.8 & 2.0 & 2.2 & -4 & 22 \\
\hline Gilchrist & 0.1 & 0.0 & 0.0 & 0.1 & 0.1 & 0.1 & 0 & 0 \\
\hline Glades & 0.0 & 0.4 & 0.0 & 0.0 & 1.5 & 0.3 & 300 & 300 \\
\hline Gulf & 28.2 & 36.2 & 33.7 & 33.9 & 32.1 & 33.5 & 19 & -1 \\
\hline Hamilton & 10.3 & 18.4 & 30.3 & 35.8 & 38.8 & 44.1 & 328 & 23 \\
\hline Hardee & 0.6 & 0.1 & 1.5 & 0.8 & 4.8 & 0.1 & -83 & -88 \\
\hline Hendry & 1.1 & 0.3 & 0.8 & 0.2 & 0.5 & 1.1 & 0 & 450 \\
\hline Hernando & 27.0 & 19.6 & 25.4 & 31.6 & 26.7 & 23.3 & -14 & -26 \\
\hline Highlands & 0.0 & 0.1 & 0.7 & 1.0 & 0.5 & 0.2 & 200 & -80 \\
\hline Hillsborough & 84.0 & 51.9 & 28.9 & 26.8 & 34.4 & 30.0 & -64 & 12 \\
\hline Holmes & 0.0 & 0.0 & 0.0 & 0.1 & 0.0 & 0.0 & 0 & -100 \\
\hline Indian River & 0.0 & 0.5 & 0.4 & 1.1 & 0.1 & 0.3 & 300 & -73 \\
\hline Jackson & 0.4 & 1.2 & 0.8 & 0.8 & 0.8 & 1.4 & 250 & 75 \\
\hline Jefferson & 0.0 & 0.2 & 0.0 & 0.1 & 0.0 & 0.1 & 100 & 0 \\
\hline Lafayette & 0.0 & 0.0 & 0.0 & 0.0 & 0.1 & 0.1 & 100 & 100 \\
\hline Lake & 19.3 & 19.4 & 20.7 & 11.2 & 15.6 & 9.5 & -51 & -15 \\
\hline Lee & 1.0 & 4.3 & 8.4 & 4.1 & 6.9 & 10.5 & 950 & 156 \\
\hline Leon & 0.0 & 0.0 & 0.1 & 1.9 & 0.3 & 0.3 & 300 & -84 \\
\hline Levy & 0.0 & 0.0 & 0.0 & 0.2 & 2.7 & 2.1 & 2,100 & 950 \\
\hline Liberty & 0.8 & 1.3 & 0.3 & 0.3 & 0.0 & 0.7 & -13 & 133 \\
\hline Madison & 0.0 & 0.0 & 0.0 & 0.1 & 0.5 & 0.2 & 200 & 100 \\
\hline Manatee & 1.3 & 3.0 & 2.0 & 0.2 & 3.3 & 2.6 & 100 & 1,200 \\
\hline Marion & 4.5 & 2.2 & 0.3 & 1.6 & 2.9 & 1.1 & -76 & -31 \\
\hline Martin & 0.5 & 0.5 & 0.1 & 0.1 & 0.2 & 29.6 & 5,820 & 29,500 \\
\hline Monroe & 0.0 & 0.0 & 0.0 & 0.0 & 0.0 & 0.1 & 100 & 100 \\
\hline Nassau & 37.8 & 50.0 & 57.9 & 45.0 & 37.4 & 32.7 & -13 & -27 \\
\hline Okaloosa & 1.4 & 4.7 & 6.1 & 4.6 & 5.8 & 6.0 & 329 & 30 \\
\hline Okeechobee & 0.0 & 0.2 & 0.0 & 0.2 & 0.1 & 0.2 & 200 & 0 \\
\hline Orange & 6.0 & 7.0 & 14.8 & 15.2 & 16.3 & 18.9 & 215 & 24 \\
\hline Osceola & 0.0 & 0.1 & 0.7 & 0.7 & 3.2 & 2.3 & 2,300 & 229 \\
\hline Palm Beach & 5.7 & 28.4 & 46.5 & 2.6 & 2.8 & 32.2 & 465 & 1,138 \\
\hline Pasco & 16.4 & 30.0 & 25.0 & 15.8 & 19.9 & 18.7 & 14 & 18 \\
\hline Pinellas & 1.0 & 2.0 & 1.3 & 0.9 & 0.1 & 0.1 & -90 & -89 \\
\hline Polk & 270.0 & 307.0 & 272.2 & 208.7 & 137.1 & 143.4 & -47 & -31 \\
\hline Putnam & 57.0 & 31.5 & 37.2 & 40.2 & 53.1 & 43.8 & -23 & 9 \\
\hline St. Johns & 0.0 & 0.0 & 2.0 & 0.0 & 0.1 & 0.1 & 100 & 100 \\
\hline St. Lucie & 0.8 & 1.2 & 0.2 & 2.1 & 2.3 & 3.1 & 288 & 48 \\
\hline Santa Rosa & 7.2 & 10.3 & 17.7 & 18.5 & 6.6 & 6.5 & -10 & -65 \\
\hline Sarasota & 0.4 & 7.6 & 3.0 & 0.1 & 0.5 & 2.4 & 500 & 2,300 \\
\hline Seminole & 0.0 & 0.5 & 2.6 & 4.1 & 5.0 & 0.5 & 500 & -88 \\
\hline Sumter & 26.0 & 18.5 & 16.1 & 1.1 & 57.8 & 60.0 & 131 & 5,355 \\
\hline Suwannee & 0.0 & 7.1 & 2.4 & 0.7 & 2.4 & 1.1 & 1,100 & 57 \\
\hline Taylor & 50.0 & 53.7 & 57.0 & 51.1 & 46.8 & 47.6 & -5 & -7 \\
\hline Union & 0.8 & 0.6 & 0.0 & 0.0 & 0.0 & 0.0 & -100 & 0 \\
\hline Volusia & 0.6 & 0.5 & 0.1 & 0.5 & 0.6 & 0.7 & 17 & 40 \\
\hline Wakulla & 1.0 & 1.1 & 1.2 & 0.8 & 0.7 & 0.8 & -20 & 0 \\
\hline Walton & 0.0 & 1.2 & 0.4 & 0.7 & 0.7 & 0.9 & 900 & 29 \\
\hline Washington & 0.0 & 0.0 & 0.0 & 0.0 & 0.0 & 0.0 & $\mathrm{~N} / \mathrm{A}$ & N/A \\
\hline Totals & 858.8 & 899.5 & 882.6 & 700.5 & 708.8 & 770.0 & -10 & 10 \\
\hline
\end{tabular}


management district from a variety of sources that included the agricultural extension agent in each county, the water management district consumptive water-use permit files, the Florida Crop and Livestock Reporting Service (Florida Department of Agriculture), the U.S. Department of Commerce, Agricultural Census, and several other minor sources. Acreage data were collected for vegetable crops (sometimes referred to as truck farming), fruit crops, field crops, and ornamentals-grasses. The second step was to determine the amount of supplemental irrigation water needed to grow each crop, as well as the water necessary to overcome the inefficiency of the irrigation system, water needed for frost and freeze protection, and water used for fertigation (the application of fertilizers and pesticides as a liquid through the irrigation system). This determination was made by each water management district, utilizing a variety of methods and information services including the modified Blaney-Criddle irrigation model, "Irrigation Water Requirements, 1970, revised" (U.S. Soil Conservation Service, 1970), the University of Florida "AFSIRS" computer model (Smajstrla, 1986), the "Florida Irrigation Guide" (U.S. Soil Conservation Service, 1982), and selected agricultural monitoring programs (Duerr and Trommer, 1982) throughout the State. Once this supplemental irrigation requirement coefficient was determined for each crop, it was then multiplied by the number of irrigated acres in each county and a wateruse value was derived.

Water withdrawals also were calculated for nonirrigation (livestock and fish farming) in two steps. First, the number of cows (including dairy cows), chickens, horses, and pigs were compiled for each county. Data were obtained from the Florida Crop and Livestock Reporting Service (poultry and dairy summaries), the agricultural extension agent in each county, the U.S. Department of Commerce, Agricultural Census, and other minor sources. The second step was to determine the amount of water used per day for each type of animal. Water-use coefficients were obtained from University of Florida, Institute of Food and Agriculture Science (St. Johns River Water Management District, 1984, p. 39) for each type of animal and then multiplied by the number of animals in each county to estimate the water needed and, hence, most likely used. Water-use estimates for fish farming were obtained from the water management district's consumptive water-use permit files, which have only been compiled since 1975 .
Agricultural irrigation and nonirrigation wateruse data for Florida were collected and compiled for 1950, 1955, 1960, 1965, 1970, 1975, 1977, 1980, 1985, and 1990. Data for these years include the amount of water withdrawn by source (ground or surface) and acres irrigated by crop type. Livestock water use values for 1950 through 1980 have been included in the agricultural irrigation totals for those years. These values originally were reported under the rural water use category that included self-supplied domestic water use. Irrigation water-use values for 1985 and 1990 include estimates for turf grass watering (including golf courses, athletic fields, cemeteries, residential and commercial lawns, public areas, and others) from non-public supply sources for some counties.

Freshwater withdrawn for agricultural purposes in Florida increased from $375 \mathrm{Mgal} / \mathrm{d}$ in 1950 to 3,805 Mgal/d in 1990 (fig. 9 and table 12). An additional $170 \mathrm{Mgal} / \mathrm{d}$ of reclaimed wastewater was used for irrigation purposes during 1990 (Marella, 1992b). More recently, water withdrawn for agriculture purposes increased nearly $780 \mathrm{Mgal} / \mathrm{d}$ (26 percent) between 1980 and 1990. Fresh ground-water withdrawals for agricultural irrigation and nonirrigation increased nearly 1,320 percent from $142 \mathrm{Mgal} / \mathrm{d}$ in 1950 to 2,013 Mgal/d in 1990 (fig. 9 and table 12).

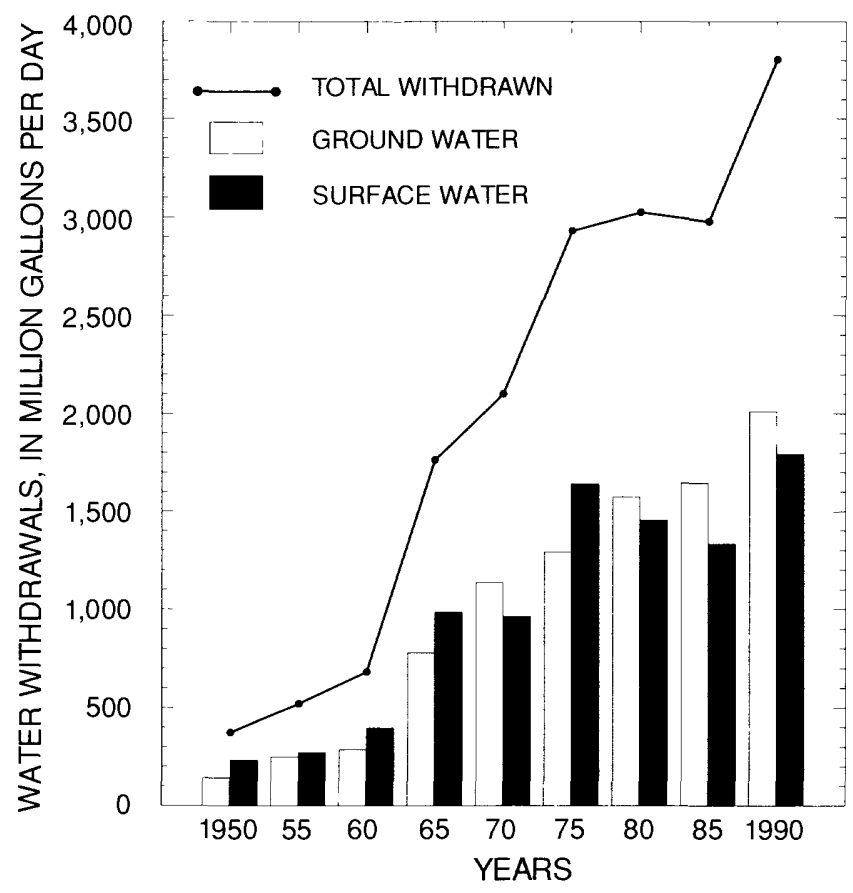

Figure 9. Agriculture (irrigation and nonirrigation) freshwater withdrawn in Florida by source, 1950-90 (modified from Marella, 1992b). 
Table 12. Agriculture acreage irrigated and freshwater withdrawals in Florida by source, 1950-90

[All withdrawal values are in million gallons per day; N/A, data not available; modified from MacKichan (1951, 1957). Florida Water Resource Commission (1956), MacKichan and Kammerer (1961), Murray (1968). Pride (1973), Leach (1978a, 1983), Leach and Healy (1980), Franks (1981), and Marella (1988a, 1992b)]

\begin{tabular}{|c|c|c|c|c|c|}
\hline \multirow[b]{2}{*}{ Year } & \multirow{2}{*}{$\begin{array}{c}\text { Acres } \\
\text { irrigated } \\
\text { (in millions) }\end{array}$} & \multicolumn{3}{|c|}{ Water withdrawn by source ${ }^{a}$} & \multirow{2}{*}{$\begin{array}{c}\text { Reclaimed } \\
\text { wastewater } \\
\text { used }\end{array}$} \\
\hline & & $\begin{array}{c}\text { Ground } \\
\text { water }\end{array}$ & $\begin{array}{c}\text { Surface } \\
\text { water }\end{array}$ & Totals & \\
\hline 1950 & $\mathrm{~N} / \mathrm{A}$ & 142.4 & 232.6 & 375.0 & N/A \\
\hline 1955 & 0.43 & 250.0 & 271.0 & 521.0 & $\mathrm{~N} / \mathrm{A}$ \\
\hline 1960 & 0.70 & 286.0 & 396.5 & 682.5 & 0.0 \\
\hline $1965^{\mathrm{b}}$ & 1.20 & 779.1 & 985.3 & 1.764 .4 & 0.0 \\
\hline 1970 & 1.60 & $1,136.3^{c}$ & $964.4^{c}$ & $2,100.7$ & 0.0 \\
\hline 1975 & 1.84 & $1,289.9$ & 1.640 .7 & $2,930.6$ & $7.5^{\mathrm{d}}$ \\
\hline 1977 & 1.93 & $1,437.3^{c}$ & $1.479 .5^{c}$ & 2.916 .8 & $8.0^{\mathrm{d}}$ \\
\hline 1980 & 2.04 & $1,572.8^{c}$ & $1,452.6^{\mathrm{c}}$ & 3.025 .4 & $11.0^{\mathrm{d}}$ \\
\hline 1985 & 1.91 & $1,646.3$ & 1.333 .0 & 2.979 .3 & 50.6 \\
\hline 1990 & 2.15 & 2.012 .5 & 1.792 .8 & $3,805.3$ & 169.6 \\
\hline
\end{tabular}

${ }^{\mathrm{a}}$ Includes water withdrawn for nonirrigation purposes that include livestock and fish farming as well as water used for irrigation and freeze protection.

${ }^{b}$ Original water withdrawal values for agricultural irrigation in 1965 were over estimated, and are adjusted in Leach, 1983.

${ }^{\mathrm{c}}$ Value has been modified from previously published number.

${ }^{\mathrm{d}}$ Values for reclaimed wastewater used for 1975, 1977, and 1980 were estimated from Franks, 1981.

The increase in ground-water withdrawals can be attributed to the need for the good quality and dependable supply that ground water can provide. Ground water also is withdrawn and used for frost and freeze protection of crops during the winter months and for the augmenting of surface-water canals, ditches, or ponds that are used for irrigation. Fresh surface-water withdrawals for agricultural irrigation increased nearly $670 \mathrm{percent}$ from $233 \mathrm{Mgal} / \mathrm{d}$ in 1950 to $1,793 \mathrm{Mgal} / \mathrm{d}$ in 1990 (table 12). The increase in surface water use resulted in part from to the draining of wetlands for development for agriculture or urban purposes and the impoundment of the diverted water for flood control as a readily available source of irrigation water. However, the quantity or quality of surface-water sources are sometimes less dependable than ground water particularly during extreme dry conditions. Furthermore, environmental concerns about wetlands and wildlife preservation require that more surface water be made available to maintain natural conditions. Additionally, many surface-water canals are strictly managed, and withdrawals or diversions from these water bodies by

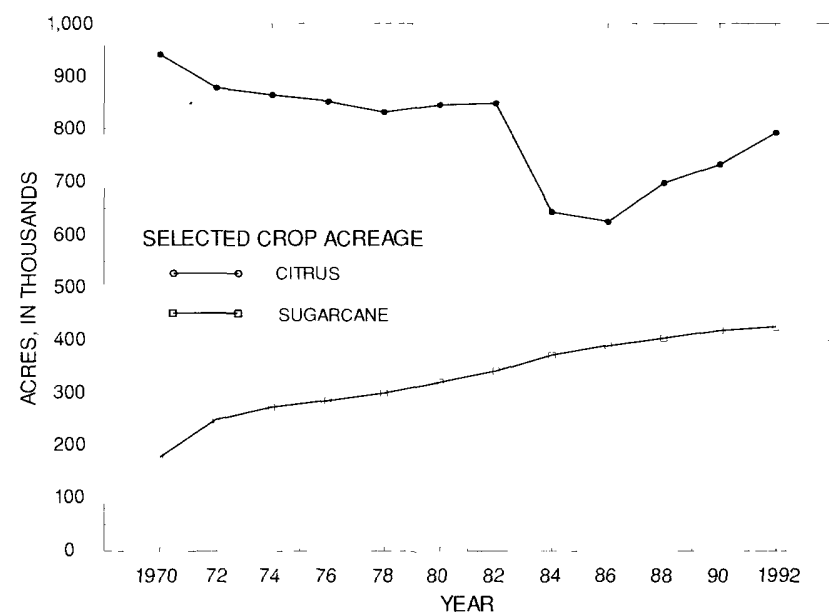

Figure 10. Citrus and sugarcane acreage in Florida, 197090 (modified from Marella, 1992b).

irrigators often are limited in duration or amount, especially during dry times. Many irrigators from these canals also supplement their irrigation water with ground water. Recent increases in surface-water withdrawals from 1980 to 1990 can be attributed to the increase in sugarcane acreage (fig. 10) (nearly all of the sugarcane in Florida is irrigated from surface-water canals), and the irrigation of various crops from tailwater runoff. Tail-water runoff refers to the unused irrigation and rainfall water from the irrigated field. This unused water is usually held in a ditch or on-site impoundment which enables the grower to reuse the water for irrigation or freeze protection. Surface water also is used for frost and freeze protection of crops during the winter months and for insect control (fields are often flooded for an extended period of time to help control insects). In addition to ground- and surfacewater withdrawals, the use of reclaimed water for irrigation increased from nearly nothing in 1970 , to $11 \mathrm{Mgal} / \mathrm{d}$ in 1980, and to $170 \mathrm{Mgal} / \mathrm{d}$ in 1990 (table 12). Agriculture has been the largest user of freshwater in Florida since 1970, and in 1990 accounted for the largest amount of water withdrawn for irrigation and nonirrigation purposes in the United States east of the Mississippi River in 1990 (Solley and others, 1993, p. 37). No saline water was known to be withdrawn for irrigation purposes in Florida.

Agriculture water use increased in 63 counties between 1965 and 1990. Original estimates for agriculture acreage and water use in 1965 were overestimated (Leach, 1983, p. 11), and many county values have recently been modified. However, between 1965 and 1990, the increase in water use was apparent as 
the irrigation technology improved and dependable water sources were tapped more efficiently. Palm Beach County accounted for the single largest amount of total agriculture withdrawals in 1965 (363 Mgal/d), and withdrawals more than doubled (115 percent) by 1990 to $779 \mathrm{Mgal} / \mathrm{d}$ (table 13). Between 1980 and 1990, agricultural irrigation and nonirrigation water use increased in 49 counties (table 13). The decrease in water use in the remaining 18 counties for this period can be attributed to a decrease of irrigated acreage due to freeze damage and urbanization, and the decrease of improved pasture irrigation. In 1980, nearly 0.50 million acres of improved pasture were estimated to have been irrigated (Leach, 1983, p. 33), compared to 0.16 million acres in 1990 (Marella, 1992b, p. 25). Since 1980, the general consensus among agricultural specialists is that the early figures for improved pasture irrigation were overestimated since the cost to pump the water generally exceeded the economic return. Therefore, during the 1980's, only the improved pasture that was irrigated by free flowing wells or gravity flood systems was thought to be irrigated. Many counties increased irrigation water use significantly between 1980 and 1990 because citrus groves were replanted in areas further south in Florida, nursery stock used for urban landscaping (mostly plants and sod) increased, and sugarcane production increased. Some of the counties heavily involved in these agricultural activities include Brevard, Collier, Dade, De Soto, Hardee, Hillsborough, Hendry, Indian River, Lee, Manatee, Martin, Osceola, Palm Beach, Polk, and St. Lucie.

The number of acres irrigated in Florida totaled nearly 2.15 million in 1990 , compared to 0.70 million in 1960 (table 12). Palm Beach, Hendry, Dade, Polk, and Brevard Counties each had more than 100,000 acres irrigated in 1990 and together accounted for 46 percent of the irrigated acreage in Florida (Marella, 1992b). Irrigated acreage in Florida continued to increase between 1980 and 1990, despite acreage losses to freezes, diseases, insect infestation, and urbanization. This increase results, in part, from the replanting of freeze-damaged acreage, in part, from more farms developing the capability to irrigate, and in part, from the market for landscape goods (plants, shrubs, and sod) created by the population and housing growth. Citrus and sugarcane are the largest individual crops irrigated in the State. In 1980 and 1990, these two crops combined, accounted for 53 percent of the irrigated acreage in Florida, and 51 percent in
1970. Sugarcane is grown in only Glades, Hendry, Martin, and Palm Beach Counties, and acreage has increased from 179,000 acres in 1970 (Pride, 1973, p. 12) to more than 400,000 in 1990 (Florida Agricultural Statistics Service, 1991) (fig. 10). The largest gain in sugarcane acreage occurred in Palm Beach County where acreage increased from 219,000 in 1975 to 313,000 in 1990 (Florida Agricultural Statistics Service, 1978 and 1991). Citrus primarily is grown in the central and southern part of Florida and the total acreage has decreased from 941,500 acres in 1970 , to 732,800 acres in 1990 (Florida Agricultural Statistical Service, 1992). However, citrus acreage was at its lowest in $1986(624,500)$ because of several damaging freezes in 1983 and 1984. Citrus acreage increased nearly 110,000 acres between 1986 and 1990 (fig. 10). Large gains in citrus acreage between 1970 and 1990 occurred in Collier, De Soto, Hendry, and St. Lucie Counties, while large losses in citrus acreage occurred in Hillsborough, Lake, Orange, Pasco, and Volusia Counties (Florida Agricultural Statistical Service, 1992, p. 13-15; Marella, 1992b, p. 30).

\section{Thermoelectric Power Generation}

The thermoelectric power generation category refers to water used for the generation of power. This category includes water used for cooling purposes, plant domestic needs, washdown or cleaning needs, and boiler makeup. Reported cooling water amounts include water withdrawn for once-through cooling, and water withdrawn to augment cooling ponds. Prior to 1975 , water-use data for this category were usually obtained by contacting the facility directly. Since 1975, water-use data for this category were collected by each water management district through their consumptive water-use permit program (compliance files), or from the monthly operating reports submitted to the FDEP. However, not all power plants are required to submit pumpage to the water management districts or FDEP, therefore data for these facilities were obtained by direct contact by the water management districts or USGS. Thermoelectric power generation water-use data for Florida were collected and compiled for 1950, 1955, 1960, 1965, 1970, 1975, 1977, 1980, 1985, and 1990. Data for these years include the amount of water withdrawn by source (ground or surface and fresh or saline) but do not include the amount of water recirculated within these facilities. Data for the amount of water supplied to 
Table 13. Agriculture freshwater use and percent change in Florida by county, 1965-90

[Modified from Pride (1973), Leach (1978a, 1983), and Marella (1988a, 1992b), includes livestock water use but does include reuse water use; values may not add to totals because of independent rounding or revisions in data]

\begin{tabular}{|c|c|c|c|c|c|c|c|c|}
\hline \multirow{2}{*}{ County } & \multicolumn{6}{|c|}{ Agricultural water use, in million gallons per day } & \multicolumn{2}{|c|}{ Percent change } \\
\hline & 1965 & 1970 & 1975 & 1980 & 1985 & 1990 & $1965-90$ & $1980-90$ \\
\hline Alachua & 3.2 & 3.4 & 7.2 & 20.3 & 15.6 & 18.5 & 478 & -9 \\
\hline Baker & 0.1 & 0.0 & 1.6 & 2.8 & 5.1 & 5.5 & 5.400 & 96 \\
\hline Bay & 0.1 & 0.3 & 0.2 & 0.7 & 2.2 & 2.3 & 2,200 & 229 \\
\hline Bradford & 0.2 & 0.1 & 0.2 & 0.9 & 1.0 & 1.1 & 450 & 22 \\
\hline Brevard & 62.0 & 47.9 & 61.9 & 166.4 & 126.0 & 111.2 & 79 & -33 \\
\hline Broward & 84.9 & 69.8 & 77.9 & 44.0 & 42.9 & 62.7 & -26 & 43 \\
\hline Calhoun & 0.4 & 0.2 & 1.4 & 1.7 & 0.4 & 0.6 & 50 & -65 \\
\hline Charlotte & 6.3 & 28.9 & 34.7 & 25.3 & 46.9 & 38.7 & 514 & 53 \\
\hline Citrus & 1.7 & 4.9 & 0.6 & 4.8 & 9.3 & 4.7 & 176 & -2 \\
\hline Clay & 5.3 & 4.1 & 0.5 & 3.6 & 3.7 & 3.4 & -36 & -6 \\
\hline Collier & 53.2 & 47.5 & 69.8 & 87.2 & 90.3 & 163.4 & 207 & 87 \\
\hline Columbia & 0.5 & 0.2 & 1.6 & 2.0 & 2.9 & 3.2 & 540 & 60 \\
\hline Dade & 67.9 & 44.8 & 90.6 & 89.7 & 121.7 & 149.9 & 121 & 67 \\
\hline De Soto & 22.7 & 64.7 & 66.7 & 35.3 & 74.5 & 108.8 & 379 & 208 \\
\hline Dixıe & 0.1 & 0.1 & 0.5 & 0.4 & 2.0 & 3.4 & 3.300 & 750 \\
\hline Duval & 0.1 & 3.7 & 2.6 & 6.2 & 20.2 & 10.9 & 10.800 & 76 \\
\hline Escambia & 0.2 & 0.1 & 4.1 & 2.5 & 5.7 & 6.4 & 3.100 & 156 \\
\hline Flagler & 3.7 & 9.2 & 8.7 & 4.2 & 7.2 & 8.7 & 1.35 & 107 \\
\hline Franklin & 0.1 & 0.0 & 0.0 & 0.0 & 1.6 & 0.9 & 800 & 900 \\
\hline Gadsden & 5.7 & 2.6 & 2.8 & 6.7 & 9.1 & 10.9 & 91 & 63 \\
\hline Gilchrist & 0.2 & 0.2 & 0.4 & 1.8 & 3.8 & 9.4 & 4.600 & 422 \\
\hline Glades & 9.6 & 46.3 & 5.35 & 124.8 & 79.8 & 80.5 & 739 & -35 \\
\hline Gulf & 0.0 & 0.0 & 0.3 & 0.1 & 4.1 & 10.1 & 10.100 & 10,000 \\
\hline Hamilton & 1.1 & 0.7 & 2.0 & 0.9 & 2.9 & 4.4 & 300 & 389 \\
\hline Hardee & 44.5 & 62.5 & 93.3 & 40.3 & 86.2 & 63.1 & 42 & 57 \\
\hline Hendry & 250.4 & 246.4 & 293.7 & 245.2 & 187.8 & 486.3 & 94 & 98 \\
\hline Hernando & 2.1 & 1.7 & 3.4 & 5.0 & 5.3 & 5.8 & 176 & 16 \\
\hline Highlands & 52.8 & 57.8 & 145.6 & 90.5 & 103.4 & 127.5 & 141 & 41 \\
\hline Hillsborough & 55.9 & 69.3 & 508 & 77.2 & 111.4 & 98.1 & 75 & 27 \\
\hline Holmes & 0.2 & 0.1 & 0.5 & 0.8 & 2.6 & 3.4 & 1.600 & 325 \\
\hline Indian River & 63.1 & 1.31 .3 & 298.2 & 278.7 & 135.6 & 167.9 & 166 & -40 \\
\hline Jackıon & 0.9 & 0.7 & 6.4 & 11.9 & 20.4 & 25.5 & 2.733 & 114 \\
\hline Jefferson & 0.2 & 0.4 & 1.7 & 1.1 & 7.7 & 10.3 & 5.050 & 836 \\
\hline Lafayctte & 0.4 & 1.1 & 28 & 2.1 & 6.8 & 8.6 & 2.050 & 310 \\
\hline Lake & 26.4 & 21.1 & 57.8 & 72.9 & 33.7 & 57.3 & 117 & -21 \\
\hline Lee & 28.2 & 35.3 & 64.4 & 46.6 & 32.1 & 100.3 & 256 & 115 \\
\hline Leon & 0.6 & 0.1 & 0.8 & 2.6 & 5.6 & 4.4 & 633 & 69 \\
\hline Levy & 1.4 & 0.4 & 1.9 & 3.0 & 12.5 & 18.8 & 1.243 & 527 \\
\hline Liberty & 0.1 & 0.0 & 0.0 & 0.1 & 0.4 & 2.3 & 2.200 & 2,200 \\
\hline Madison & 0.7 & 1.7 & 2.1 & 2.5 & 2.5 & 3.2 & 357 & 28 \\
\hline Manatee & 38.2 & 49.5 & 258 & 68.6 & 89.9 & 95.5 & 150 & 39 \\
\hline Marion & 9.2 & 6.8 & 18.9 & 21.3 & 23.0 & 16.6 & 80 & -22 \\
\hline Martin & 55.6 & 90.3 & 84.1 & 139.4 & 147.2 & 137.7 & 148 & -1 \\
\hline Monroe & 0.0 & 0.0 & 00 & 0.5 & 1.6 & 1.2 & 1.200 & 140 \\
\hline Nassau & 0.9 & 0.0 & 09 & 2.5 & 2.7 & 3.0 & 233 & 20 \\
\hline Okaloosa & 0.3 & 0.0 & 09 & 0.3 & 2.3 & 1.9 & 533 & 533 \\
\hline Okeechobee & 38.2 & 34.8 & 85.0 & 88.1 & 26.1 & 40.5 & 6 & -54 \\
\hline Orange & 17.6 & 20.1 & 33.0 & 67.5 & 100.8 & 96.8 & 450 & 43 \\
\hline Osceola & 16.2 & 13.4 & 1.3 .1 & 22.1 & 45.6 & 58.1 & 259 & 163 \\
\hline Palm Beach & 362.6 & 409.8 & 505.4 & 615.3 & 553.3 & 778.7 & 115 & 27 \\
\hline Pasco & 15.6 & 9.6 & 50.4 & 22.0 & 23.3 & 21.4 & 37 & -3 \\
\hline Pinellas & 12.8 & 4.0 & 34.3 & 9.9 & 10.8 & 6.4 & -50 & -35 \\
\hline Polk & 60.9 & 173.4 & 102.0 & 57.5 & 118.3 & 133.2 & 119 & 132 \\
\hline Putnam & 13.3 & 9.7 & 19.0 & 36.9 & 18.2 & 19.1 & 44 & -48 \\
\hline St. Johns & 14.0 & 22.1 & 28.9 & 25.3 & 42.1 & 41.9 & 199 & 66 \\
\hline St. Lucie & 185.7 & 185.4 & 368.9 & 232.0 & 212.4 & 225.5 & 21 & -3 \\
\hline Santa Rosa & 0.4 & 0.2 & 0.6 & 1.0 & 1.8 & 6.2 & 1,450 & 520 \\
\hline Sarasota & 40.8 & 29.6 & 20.7 & 21.4 & 20.1 & 35.0 & -14 & 64 \\
\hline Seminole & 10.9 & 6.1 & 11.0 & 19.1 & 25.3 & 13.0 & 19 & -32 \\
\hline Sumter & 4.2 & 4.6 & 4.2 & 17.4 & 17.1 & 8.7 & 107 & -50 \\
\hline Suwannee & 1.0 & 4.1 & 2.0 & 9.6 & 16.7 & 27.8 & 2,680 & 190 \\
\hline Taylor & 0.2 & 0.1 & 0.4 & 0.3 & 0.6 & 0.7 & 250 & 133 \\
\hline Union & 0.2 & 0.1 & 0.3 & 0.3 & 0.7 & 2.0 & 900 & 567 \\
\hline Volusia & 7.8 & 7.2 & 5.6 & 25.4 & 43.1 & 26.7 & 242 & 5 \\
\hline Wakulla & 0.0 & 0.0 & 0.0 & 0.1 & 0.0 & 0.1 & 100 & 0 \\
\hline Walton & 0.2 & 10.1 & 1.0 & 9.3 & 2.4 & 4.1 & 1.950 & -56 \\
\hline Washington & 0.3 & 0.0 & 0.2 & 0.1 & 1.0 & 0.5 & 67 & 400 \\
\hline Totals & $1,764.4$ & $2,100.7$ & $2,930.6$ & $3,025.4$ & 2.979 .3 & $3,805.3$ & 116 & 26 \\
\hline
\end{tabular}


power plants by public supply and the amount of power produced were collected and compiled for selected years only. Surface-water withdrawals for 1950 were not delineated between fresh or saline. Power-generation data also were collected for the two hydroelectric plants in Florida. Some thermoelectric power generation water-use values have been adjusted or modified from previously published data. Most of the modifications were a result of changing self-supplied ground-water withdrawals to deliveries from public supply and reclassifying the source of several withdrawals from fresh to saline water. Several counties reported ground-water withdrawn for thermoelectric power generation for selected years; however, recent information provided by the power plants indicate that no ground water was withdrawn at these facilities. Any water used at these facilities other than surface water, was supplied by a public-supply water system. Additionally, several facilities that used selfsupplied water, only provided surface water (cooling water) values, and did not report ground water usage. For these facilities, self-supplied ground water values were obtained and updated.

Water withdrawn for thermoelectric power generation increased from $1,430 \mathrm{Mgal} / \mathrm{d}$ in 1955 to $11,042 \mathrm{Mgal} / \mathrm{d}$ in 1990 (fig. 11). The 1950 value of

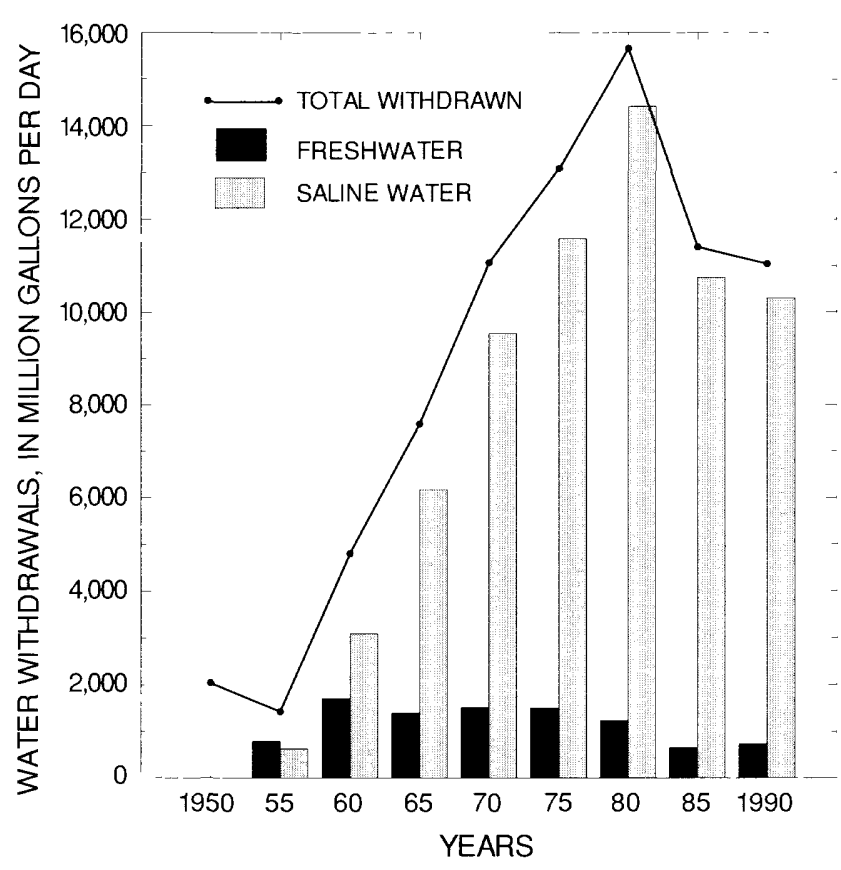

Figure 11. Thermoelectric power generation water withdrawn in Florida by source, 1950-90 (values for 1950 were not differentiated between fresh and saline; modified from Marella, 1992b).
2,047 Mgal/d was not subdivided into fresh or saline totals, and also may include some estimates of water used for power generation at some industrial facilities. Overall, freshwater withdrawals for thermoelectric power generation decreased 8 percent between 1955 and 1990 , however, withdrawals generally increased between 1955 and 1980 (fig. 11 and table 14). Saline water withdrawals for thermoelectric power generation increased 1,540 percent between 1955 and 1990, however, withdrawals generally decreased between 1980 and 1990 (fig. 11 and table 14). Fresh ground-water withdrawals increased from less than $1 \mathrm{Mgal} / \mathrm{d}$ in 1955 to more than $23 \mathrm{Mgal} / \mathrm{d}$ in 1990 (table 14). However, fresh groundwater withdrawals only accounted for 3 percent of the freshwater use in 1990. Fresh surface-water withdrawals increased significantly between 1955 and 1975, but generally decreased after 1975 (table 14) despite the addition of several new power plants. During the 1970's and early 1980 's several power plants converted from withdrawing once-through cooling water directly from a freshwater source to recirculating the cooling water through a pond or impoundment. The amount of water needed to operate these facilities did not change, however, the water withdrawn decreased significantly, as only water needed to augment or supplement the pond or impoundment was withdrawn from the original source. Additionally, between 1970 and 1990, most new powerplants were built in coastal areas to utilize saline water for oncethrough cooling purposes, or were built with cooling towers instead of cooling ponds. The cooling towers allow for the water used to cool the steam in the condenser to be sprayed into the top of the tower, thus exposing the water droplets to the cooling action of evaporation as they fall. The water is then collected at the bottom, were it is used again. Water withdrawn at these facilities also is used only to augment the cooling water. Saline water withdrawals experienced the similar trend as freshwater. Withdrawals increased through the 1970's and decreased through the 1980's (table 14) as some of the older powerplants in coastal areas were phased down in production, closed, or used only for stand by purposes. Additionally, some changes between 1970 and 1990 may have resulted from reclassifying water sources at several coastal facilities from freshwater to saline water. Several power plants are located along rivers that are tidally effected by the Atlantic Ocean or the Gulf of Mexico (McPherson and Hammett, 1991). Typically, the water used for oncethrough cooling at these facilities are a mix of fresh and saline that changes daily and seasonally. In these cases, the water was classified as saline for consistency. 
Table 14. Thermoelectric power generation water withdrawals in Florida by source, 1950-90

[All withdrawal values are in million gallons per day; Gwh, gigawatthour; N/A, data not available; modified from MacKichan (1951, 1957), MacKichan and Kammmerer (1961), Murray (1968), Murray and Reeves (1972), Pride (1973). Leach (1978a, 1983), Leach and Healy (1980). Marella (1988a, 1992b), and Solley and others $(1988,1993)]$

\begin{tabular}{|c|c|c|c|c|c|c|c|}
\hline \multirow[b]{2}{*}{ Year } & \multirow{2}{*}{$\begin{array}{c}\text { Power } \\
\text { generated } \\
\text { in Gwh }\end{array}$} & \multicolumn{3}{|c|}{ Freshwater withdrawn by source } & \multicolumn{3}{|c|}{ Saline water withdrawn by source } \\
\hline & & $\begin{array}{c}\text { Ground } \\
\text { water }\end{array}$ & $\begin{array}{c}\text { Surface } \\
\text { water }\end{array}$ & $\begin{array}{l}\text { Totals } \\
\text { water }\end{array}$ & $\begin{array}{c}\text { Ground } \\
\text { water }\end{array}$ & $\begin{array}{c}\text { Surface } \\
\text { water }\end{array}$ & Totals \\
\hline $1950^{\mathrm{a}}$ & $\mathrm{N} / \mathrm{A}$ & 0.0 & 0.0 & 0.0 & 0.0 & 0.0 & 0.0 \\
\hline 1955 & N/A & 0.0 & 800.0 & 800.0 & 0.0 & 630.0 & 630.0 \\
\hline 1960 & $\mathrm{~N} / \mathrm{A}$ & 8.0 & $1,700.0$ & $1,708.0$ & 0.0 & $3,100.0$ & $3,100.0$ \\
\hline 1965 & $\mathrm{~N} / \mathrm{A}$ & 7.2 & $1,401.0$ & $1,408.2$ & 80.0 & $6,100.0$ & $6,180.0$ \\
\hline 1970 & 57.3 & $4.5^{\mathrm{b}}$ & $1,515.0^{\mathrm{b}}$ & $1,519.5$ & 50.1 & $9,500.0^{\mathrm{b}}$ & $9,550.1$ \\
\hline 1975 & 81.1 & $14.3^{b}$ & $1,488.8^{b}$ & $1,503.1$ & 47.5 & $11,536.1^{\mathrm{b}}$ & $11,583.6$ \\
\hline 1977 & 134.9 & $16.4^{\mathrm{b}}$ & $1,216.6^{\mathrm{b}}$ & $1,233.0$ & 49.3 & $14,782.2^{b}$ & $14,831.5$ \\
\hline 1980 & 129.4 & $19.8^{b}$ & $1,219.1^{b}$ & $1,238.9$ & 79.0 & $14,334.3^{b}$ & $14,413.3$ \\
\hline 1985 & 95.2 & 18.7 & 632.7 & 651.4 & 59.1 & $10,695.0$ & $10,754.1$ \\
\hline 1990 & 128.3 & 23.1 & 708.9 & 732.0 & 49.3 & $10,260.5$ & $10,309.8$ \\
\hline
\end{tabular}

${ }^{\mathrm{a}}$ Water withdrawals for thermoelectric power generation in 1950 totaled 2,047.0 million gallons per day (MacKichan, 1951, p. 6), however, the amount of fresh and saline water was not delineated.

${ }^{b}$ Value has been modified from previously published number.

A total of 57 powerplants were operating in Florida during 1990, this included 55 thermoelectric facilities and 2 hydroelectric facilities (Marella, 1992b, p. 27). This compares to the 54 thermoelectric facilities in 1985 and 51 thermoelectric facilities in 1980. Although the number of powerplants in Florida has only increased slightly, the power production capacity increased substantially during 1970 to 1990 , because several powerplants were upgraded by added additional generating capacities and the powerplants built after 1970 had much more power generation capacity than the facilities built prior to 1970 (Ron Hix, Florida Power and Light, West Palm Beach, oral commum., 1994). The generating capacity of the new powerplants and the renovated powerplants allowed power companies to close or use for stand by purposes the older, inefficient, and smaller facilities. Gross power generated at the 57 powerplants in Florida for 1990 totaled 128.3 gigawatthours (Gwh) (Marella, 1992b, p. 29), compared to $95.5 \mathrm{Gwh}$ in 1985 (Solley and others, 1988, p. 41) and 129.4 Gwh in 1980 (Leach, 1983, p. 40) (table 14). The reader is cautioned when comparing values for power generation in Florida, as not all of the facilities may have been accounted for or the collected data may not have been differentiated between gross or net power generated.

Freshwater withdrawn for self-supplied thermoelectric power generation occurred in only 27 counties in 1990, compared to 22 counties in 1965 (table 15). Most of the water use increases in several counties between 1965 and 1990 are a result of the operation of new power generation facilities, while most of the decreases resulted from changes in cooling methods at existing plants or the down sizing (decreasing power production) of some of the older plants. Additional differences between years could have occurred due to significant facility down time caused by plant maintenance or modernization. These breaks in operation often can last for an extended period of time, and can reduce significantly the annual average water withdrawn. For example, a decline in water use for thermoelectric power generation within a county may be a result of a temporary plant closure, as opposed to a change in cooling methods. This fluctuation in water-use values is particularly evident when data are collected only every five years. 
Table 15. Thermoelectric power generation freshwater use and percent change in Florida by county, 1965-90

[Modified from Pride (1973), Leach (1978a, 1983), and Marella (1988a, 1992b); includes water withdrawn for once-through cooling purposes; values may not add to totals because of independent rounding or revisions in data; N/A, not applicable]

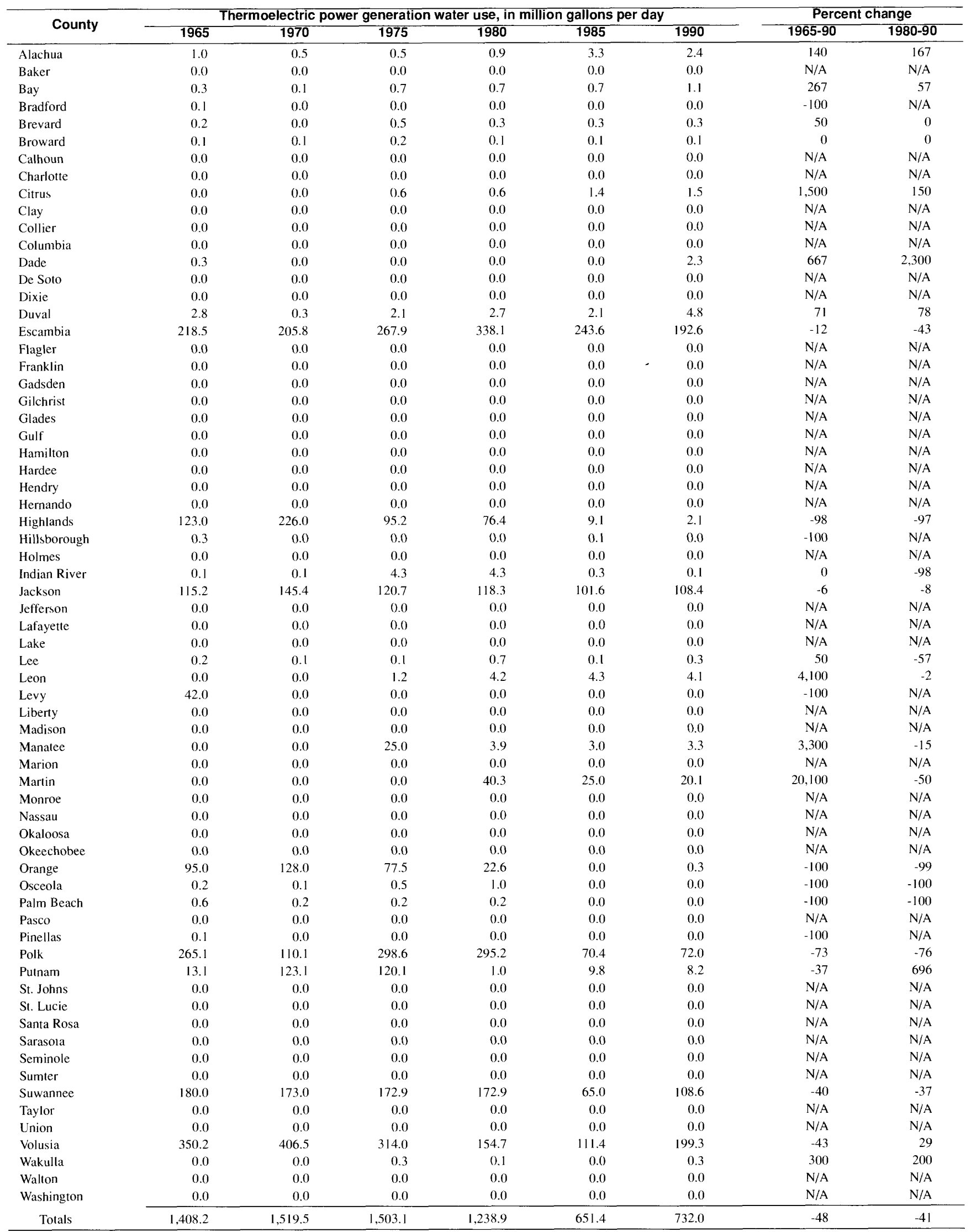


Several municipalities have small publiclyowned power-generating facilities. Most of these facilities withdraw little or no water, but use publicsupplied water. Public-supply deliveries to all thermoelectric powerplants during 1990 totaled nearly $6 \mathrm{Mgal} / \mathrm{d}$ (Marella, 1992b, p. 27), compared to $4 \mathrm{Mgal} / \mathrm{d}$ in 1980 (Leach, 1983, p. 43). Most of this water was used for domestic purposes throughout the plant or used as boiler makeup water. An additional $4 \mathrm{Mgal} / \mathrm{d}$ of reclaimed water was used primarily as cooling water at four plants in 1990 (Marella, 1992b, p. 27). However, much more reclaimed wastewater was discharged to lakes or ponds that also are used for powerplant cooling.

\section{WATER USE IN FLORIDA BY COUNTY, 1965-90}

Since 1965, water-use data have been delineated and reported for all 67 counties in Florida (fig. 2). County data in Florida have been reported for the following general categories; public supply, selfsupplied domestic, self-supplied commercial-industrial, agriculture (irrigation and nonirrigation), and thermoelectric power generation. For the years 1965, 1970, 1975, 1977, 1980, 1985, and 1990 water-use data were compiled for all categories for all counties in Florida. Most of these years correspond to years during which the USGS compiled water-use data for its national assessment. For all other years, water-use data on a county level were collected and compiled sporadically. Since 1975 , the collection and compilation of water-use data have mostly depended on the five water management districts. Between 1977 and 1990, only two water management districts (St. Johns River and Southwest Florida) have collected and compiled water-use data annually for the counties within their jurisdiction. However, these data have generally only been collected and compiled for the part of the county within that water management district, and therefore, complete water-use totals do not exist for those subdivided counties. Additional county data were collected and compiled for public supply for the years of 1978, 1986, 1987, 1988, and 1989 by the USGS. Aside from these areas and years, water-use data are not available for most counties between 1970 and 1990 (table 16).

All of the freshwater use data collected (published and unpublished) for each county between 1965 and 1990 are presented in the Appendix, tables 1 (Alachua County) through 67 (Washington County). These tables present freshwater use (withdrawals) for ground and surface water for each of the following categories; public supply, self-supplied domestic, selfsupplied commercial-industrial, agriculture, and thermoelectric power generation. For the readers convenience, the data sources are included as footnotes at the bottom of each of the County summary tables. Additionally, data that had been collected but not previously published are noted and listed by the agency of origination.

Due to current knowledge or updated information, some of the county water-use values have been modified in these tables and modifications are noted. The modifications can be classified into several categories. For public supply, most modifications were a result of eliminating the double counting of water withdrawals for several utilities in some counties or adding utilities that were missed in the original inventory. For commercial or industrial, most modifications were a result of adding missing users, reclassifying cooling water under thermoelectric, or deleting water withdrawals for heat exchange units through a closed loop system. For agriculture most of the changes were a result of adjusting for the overestimation of the 1965 data. For thermoelectric power generation, most of the modifications were a result of changing selfsupplied ground-water withdrawals to deliveries from public supply and reclassifying the source of several withdrawals from fresh to saline water.

\section{WATER USE IN FLORIDA BY WATER MANAGEMENT DISTRICT, 1975-90}

The Florida Water Resource Act of 1972 established authority for management of the water resources for Florida through five water management districts under the general supervision of the Florida Department of Natural Resources (Fernald and Patton, 1984). These districts, encompassing the entire State, are the Northwest Florida Water Management District, the St. Johns River Water Management District, the South Florida Water Management District, the Southwest Florida Water Management District, and the Suwannee River Water Management District (fig. 12). The counties located within each water management district are listed on table 17. The districts are empowered to oversee a variety of water issues on a regional level and are currently under the supervision of the FDEP (Marella, 1990b, p. 213-214). Due to current knowledge or updated information, some of the water management districts water-use values have been modified and may not precisely equal those published in the reports by the water management districts. 


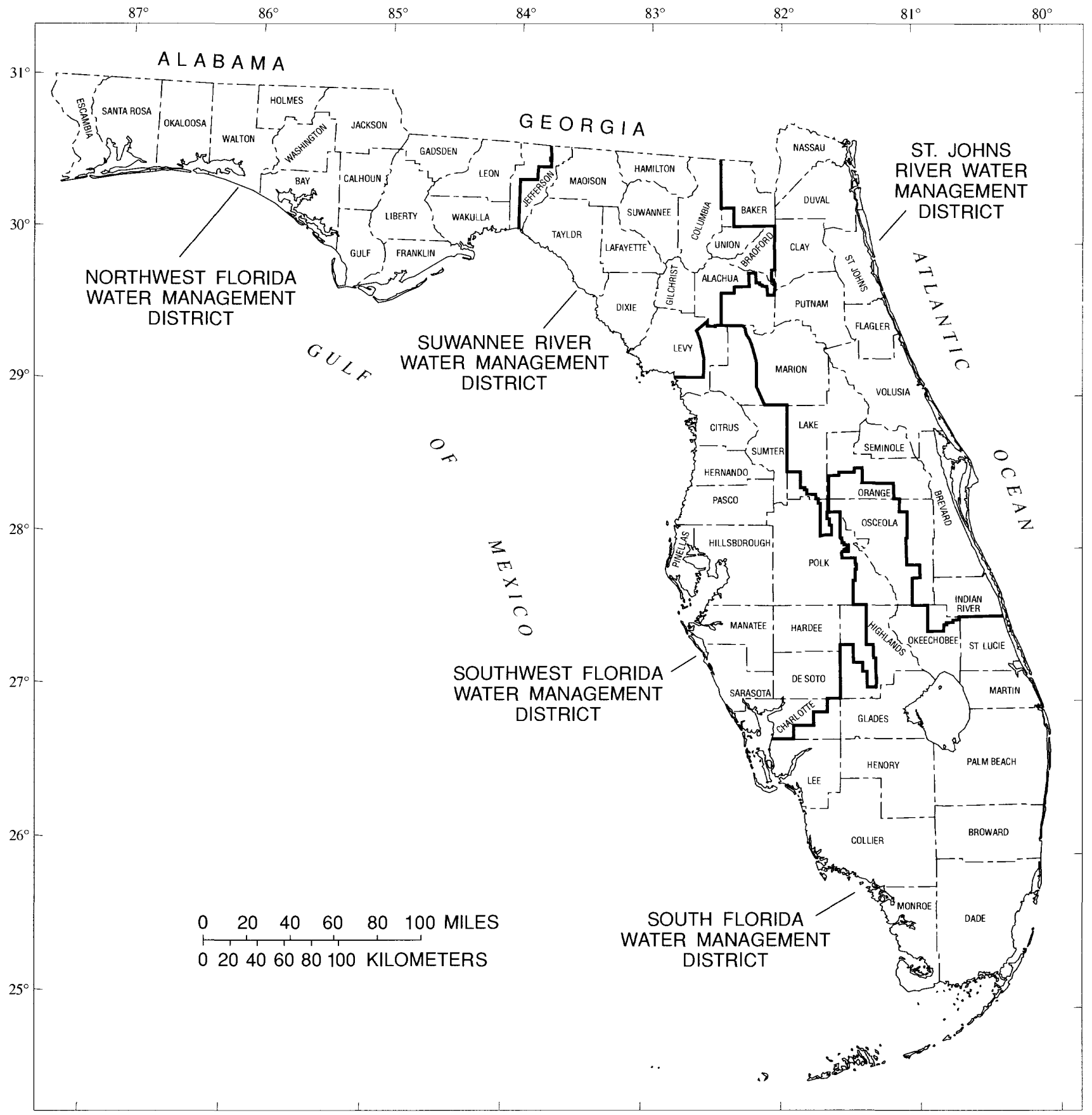

Figure 12. Boundaries of water management districts (from Marella, 1993).

Since 1975 , the water management districts have been involved in water-use data collection and tabulation. By 1980, all of the districts were collecting such data through a direct funding program with the USGS. By 1985, the direct federal funding was discontinued, and the water-use programs at the districts became fragmented and sporadic. Only the St. Johns River and the Southwest Florida Water Management Districts continued an active water-use program.
However, for the 1985 and 1990 State data collection effort, the Northwest Florida, the South Florida, and the Suwannee River Water Management Districts were instrumental in compiling the data necessary for their districts. This section provides the water-use data that were collected for each water management district for the years between 1975 and 1990. Since the districts inception during the early 1970's, the South Florida Water Management District has 
Table 17. Counties within the water management districts in Florida, 1990

[" $x "$ indicates that the county is within the district]

\begin{tabular}{|c|c|c|c|c|c|c|c|c|c|c|c|}
\hline County & $\begin{array}{c}\text { Northwest } \\
\text { Florida }\end{array}$ & $\begin{array}{l}\text { St. Johns } \\
\text { River }\end{array}$ & $\begin{array}{c}\text { South } \\
\text { Florida }\end{array}$ & $\begin{array}{l}\text { Southwest } \\
\text { Florida }\end{array}$ & $\begin{array}{c}\text { Suwannee } \\
\text { River }\end{array}$ & County & $\begin{array}{l}\text { Northwest } \\
\text { Florida }\end{array}$ & $\begin{array}{c}\text { St. Johns } \\
\text { River }\end{array}$ & $\begin{array}{l}\text { South } \\
\text { Florida }\end{array}$ & $\begin{array}{l}\text { Southwest } \\
\text { Florida }\end{array}$ & $\begin{array}{c}\text { Suwannee } \\
\text { River }\end{array}$ \\
\hline Alachua & & $x$ & & & $\mathrm{x}$ & Lake & & $\mathrm{x}$ & & $x$ & \\
\hline Baker & & $\mathrm{x}$ & & & $\mathrm{x}$ & Lee & & & $x$ & & \\
\hline Bay & $\mathrm{x}$ & & & & & Leon & $\mathrm{x}$ & & & & \\
\hline Bradford & & $\mathrm{x}$ & & & $x$ & Levy & & & & $\mathrm{x}$ & $\mathrm{x}$ \\
\hline Brevard & & $\mathrm{x}$ & & & & Liberty & $x$ & & & & \\
\hline Broward & & & $x$ & & & Madison & & & & & $x$ \\
\hline Calhoun & $x$ & & & & & Manatee & & & & $x$ & \\
\hline Charlotte & & & $x$ & $x$ & & Marion & & $\mathrm{x}$ & & $\mathrm{x}$ & \\
\hline Citrus & & & & $\mathrm{x}$ & & Martin & & & $\mathrm{x}$ & & \\
\hline Clay & & $x$ & & & & Monroe & & & $x$ & & \\
\hline Collier & & & $\mathrm{x}$ & & & Nassau & & $x$ & & & \\
\hline Columbia & & & & & $x$ & Okaloosa & $\mathrm{x}$ & & & & \\
\hline Dade & & & $x$ & & & Okecchobee & & $x$ & $x$ & & \\
\hline De Soto & & & & $x$ & & Orange & & $x$ & $x$ & & \\
\hline Dixie & & & & & $x$ & Osceola & & $\mathrm{x}$ & $x$ & & \\
\hline Duval & & $x$ & & & & Palm Beach & & & $x$ & & \\
\hline Escambia & $x$ & & & & & Pasco & & & & $x$ & \\
\hline Flagler & & $x$ & & & & Pinellas & & & & $x$ & \\
\hline Franklin & $\mathrm{x}$ & & & & & Polk & & $x$ & $\mathrm{x}$ & $x$ & \\
\hline Gadsden & $x$ & & & & & Putnam & & $x$ & & & $x$ \\
\hline Gilchrist & & & & & $\mathrm{x}$ & St. Johns & & $x$ & & & \\
\hline Glades & & & $x$ & & & St. Lucie & & & $x$ & & \\
\hline Gulf & $x$ & & & & & Santa Rosa & $\mathrm{x}$ & & & & \\
\hline Hamilton & & & & & $x$ & Sarasota & & & & $x$ & \\
\hline Hardee & & & & $\mathrm{x}$ & & Seminole & & $\mathrm{x}$ & & & \\
\hline Hendry & & & $\mathrm{x}$ & & & Sumter & & & & $\mathrm{x}$ & \\
\hline Hernando & & & & $x$ & & Suwannee & & & & & $x$ \\
\hline Highlands & & & $x$ & $x$ & & Taylor & & & & & $\mathrm{x}$ \\
\hline Hillsborough & & & & $\mathrm{x}$ & & Union & & & & & $x$ \\
\hline Holmes & $x$ & & & & & Volusia & & $\mathrm{x}$ & & & \\
\hline Indian River & & $x$ & & & & Wakulla & $x$ & & & & \\
\hline Jackson & $x$ & & & & & Walton & $x$ & & & & \\
\hline Jefferson & $x$ & & & & $x$ & Washington & $x$ & & & & \\
\hline Lafayette & & & & & $\mathrm{x}$ & & & & & & \\
\hline
\end{tabular}

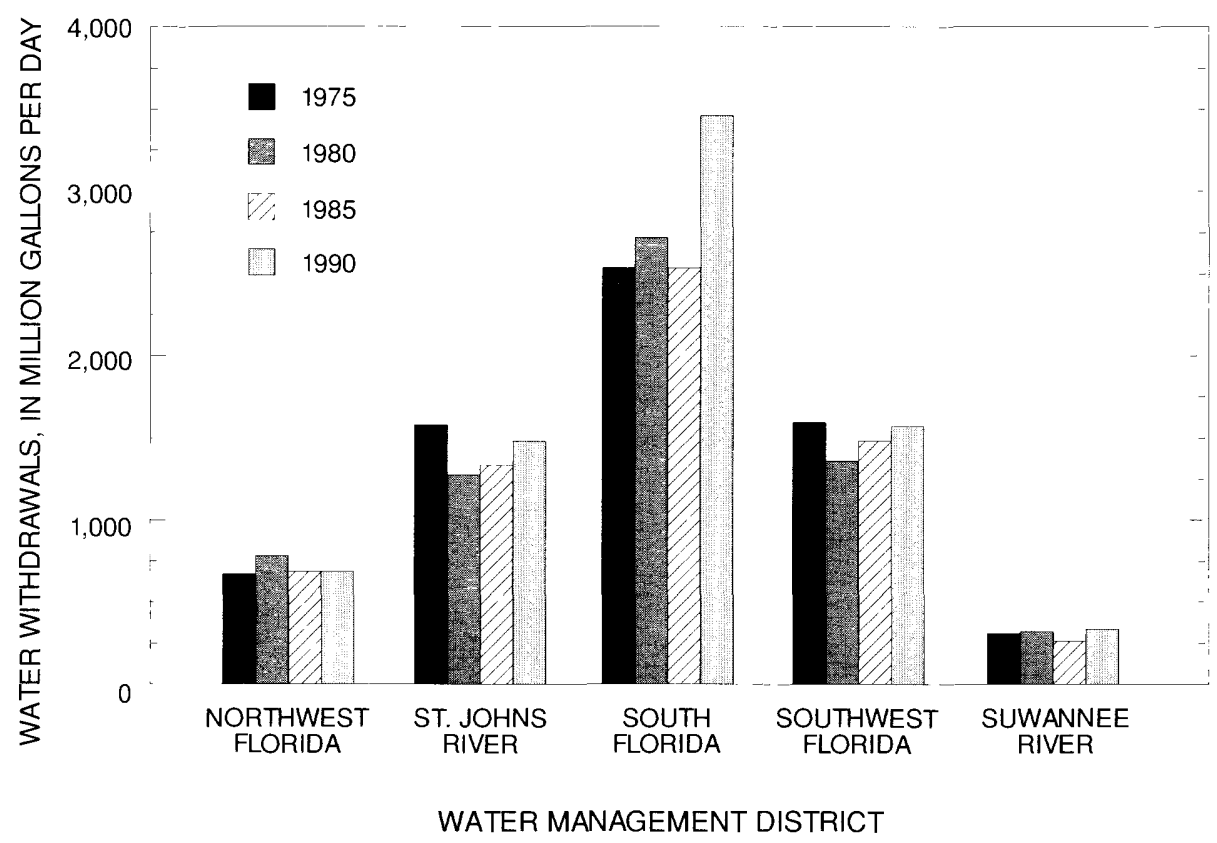

Figure 13. Freshwater withdrawn by water management district, 1975-90 (modified from Leach, 1978a, 1983, and Marella, 1992b). 
accounted for the largest amount of freshwater used in the State (fig. 13). The South Florida Water Management District accounted for 46 percent of the total freshwater used in Florida during 1990, an increase from the 38 percent in 1975. Detailed information on the amount of water withdrawn and water-use trends within each water management district between 1975 and 1990 are provided in this section.

\section{The Northwest Florida Water Management District}

The Northwest Florida Water Management District is located in the western panhandle of Florida, and encompasses approximately 19 percent $(11,200$ square miles) of the total land area of the State (Fernald and Patton, 1984, p. 198). Located within the district are all or part of the following 16 counties; Bay, Calhoun, Escambia, Franklin, Gadsden, Gulf, Holmes, Jackson, Jefferson (part), Leon, Liberty, Okaloosa, Santa Rosa, Wakulla, Walton, and Washington (fig. 12 and table 17). In 1990, the district had an estimated population of nearly 1.01 million residents (Marella, 1992b, p. 29), or approximately 8 percent of the total resident population of Florida.
This represents nearly a 30 percent increase in population from the 0.77 million in 1975 (Leach, 1978a, p. 47). Total freshwater withdrawals within the district increased about 3 percent between 1975 and 1990. Specifically, fresh ground-water withdrawals increased from $172 \mathrm{Mgal} / \mathrm{d}$ in 1975 to $271 \mathrm{Mgal} / \mathrm{d}$ in 1990, or 58 percent (fig. 14 and table 18). This increase primarily is due to an increase in publicsupply withdrawals that is related to the population growth and tourism between 1975 and 1990. Nearly 75 percent of the public supply water for the Northwest Florida Water Management District was obtained from ground-water sources during 1990. Fresh surface-water withdrawals decreased from $496 \mathrm{Mgal} / \mathrm{d}$ in 1975 to $415 \mathrm{Mgal} / \mathrm{d}$ in 1990 , or 16 percent (fig. 14 and table 18). Over 90 percent of the decrease in fresh surface-water withdrawals between 1975 and 1990 was a result of lower usage for thermoelectric power generation. The Northwest Florida Water Management District accounted for 9 percent of the State's total freshwater use in 1990 , compared to 10 percent in 1975.

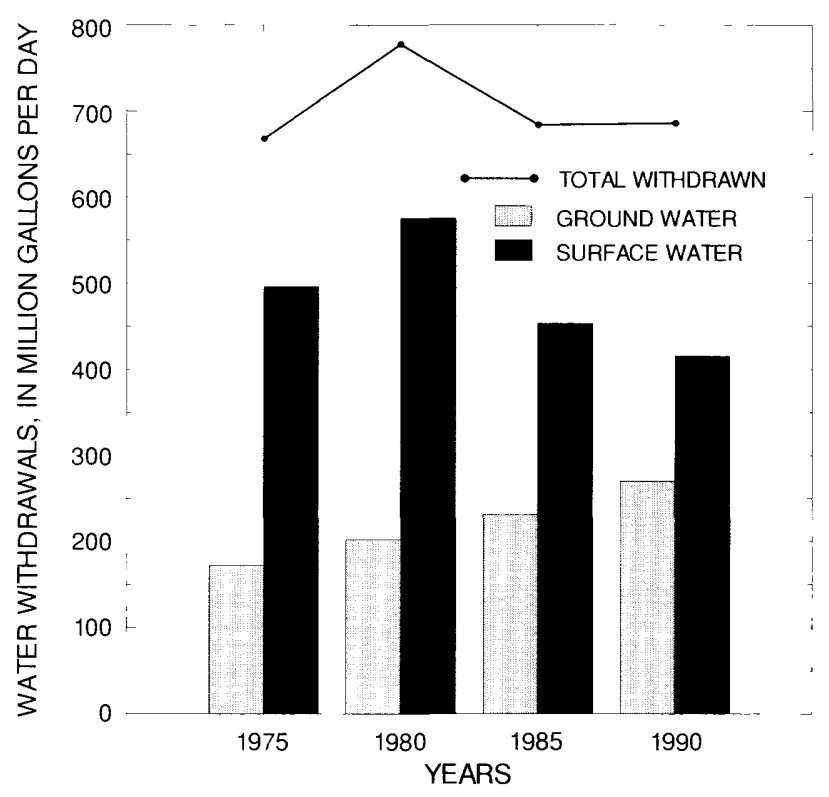

Figure 14. Freshwater withdrawin in the Northwest Florida Water Management District by source, 1975, 1980, 1985 and 1990 (modified from Leach, 1978a, 1983, Bielby, 1987, and Marella, 1992b). 


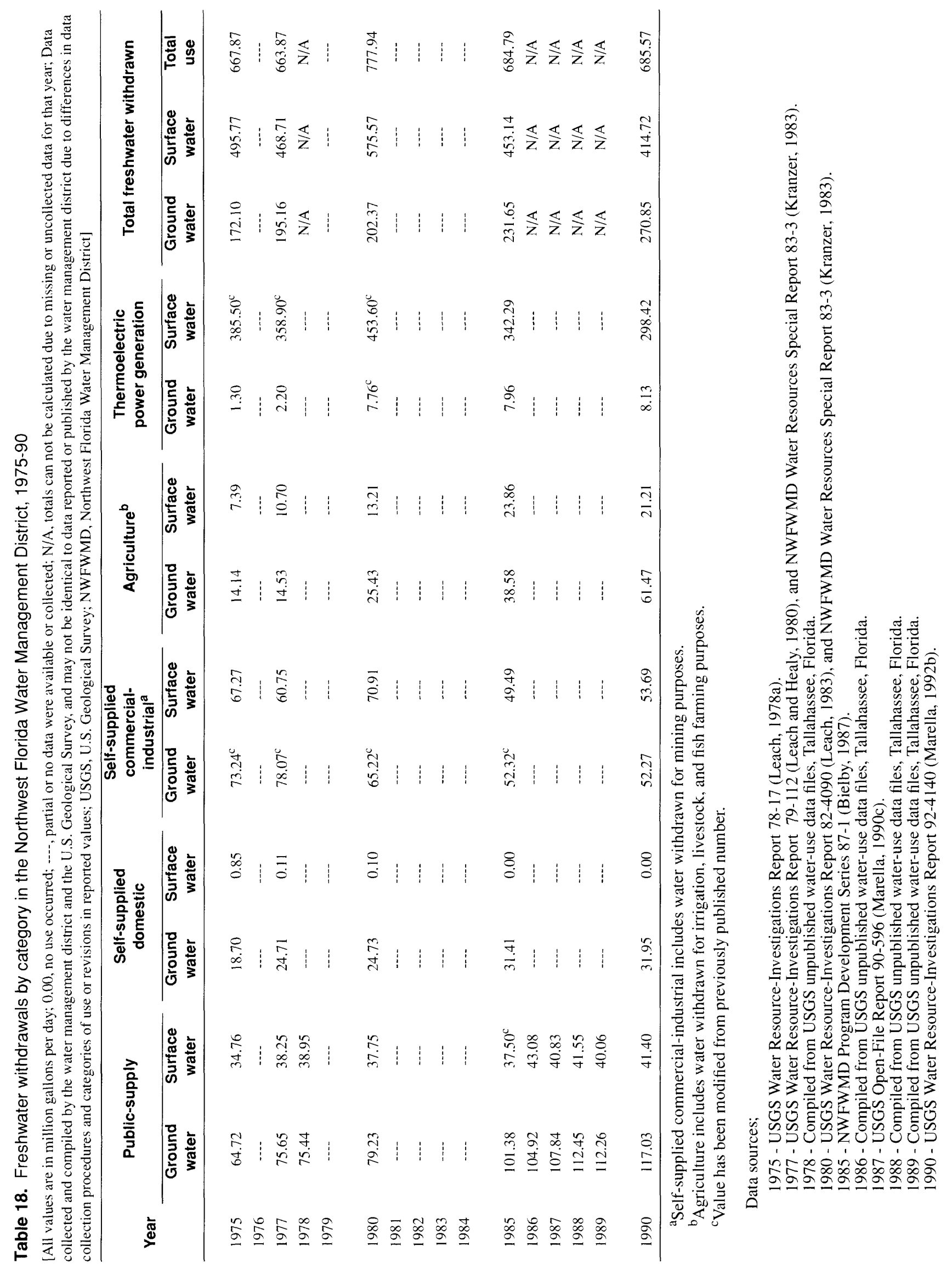




\section{The St. Johns River Water Management District}

The St. Johns River Water Management District is located in the northeast and central area of Florida, and encompasses approximately 21 percent $(12,400$ square miles) of the total land area for the State (Fernald and Patton, 1984, p. 158). Located within the district are all or part of the following 19 counties; Alachua (part), Baker (part), Bradford (part), Brevard, Clay, Duval, Flagler, Indian River, Lake (part), Marion (part), Nassau, Okeechobee (part), Orange (part), Osceola (part), Polk (part), Putnam (part), St. Johns, Seminole, and Volusia (fig. 12 and table 17). In 1990, the water management district had an estimated population of nearly 3.17 million residents (Marella, 1992b, p. 29), or approximately 24.5 percent of the total resident population of Florida. This represents a 54 percent increase in population from the 2.06 million in 1975 (Leach, 1978a, p. 48). Total freshwater withdrawals within the district decreased about 6 percent between 1975 and 1990.
Specifically, fresh ground-water withdrawals increased from $715 \mathrm{Mgal} / \mathrm{d}$ in 1975 to $1,021 \mathrm{Mgal} / \mathrm{d}$ in 1990, or 43 percent (fig. 15 and table 19). This increase primarily is due to an increase in publicsupply withdrawals that is related to the population growth and a high seasonal population between 1975 and 1990. More than 96 percent of the public-supply water for the St. Johns River Water Management District was obtained from ground-water sources during 1990. Additional increases in ground-water withdrawals within the district occurred due to an increase in acreage irrigated for agriculture. Fresh surface-water withdrawals decreased by 47 percent from $864 \mathrm{Mgal} / \mathrm{d}$ in 1975 to $458 \mathrm{Mgal} / \mathrm{d}$ in 1990 (fig. 15 and table 19). Over 75 percent of the decrease in fresh surface-water withdrawals between 1975 and 1990 was a result of lower usage for thermoelectric power generation. The St. Johns River Water Management District accounted for 20 percent of the State's total freshwater use in 1990 , compared to 23 percent in 1975.

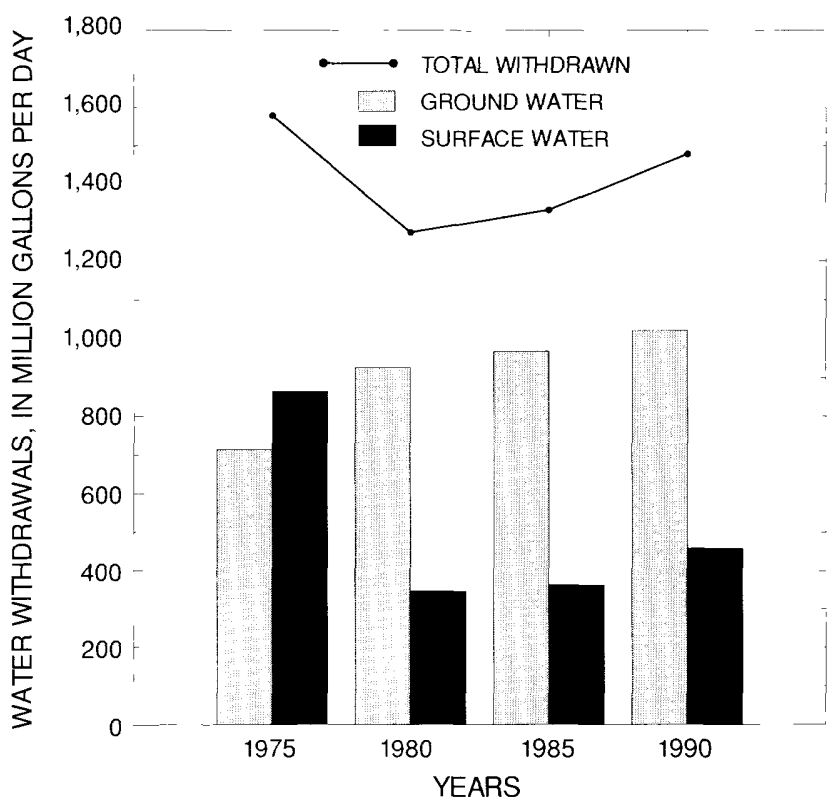

Figure 15. Freshwater withdrawn in the St. Johns River Water Management District by source, 1975, 1980, 1985 and 1990 (modified from Leach, 1978a, and Marella, 1986, 1987a, 1992b). 


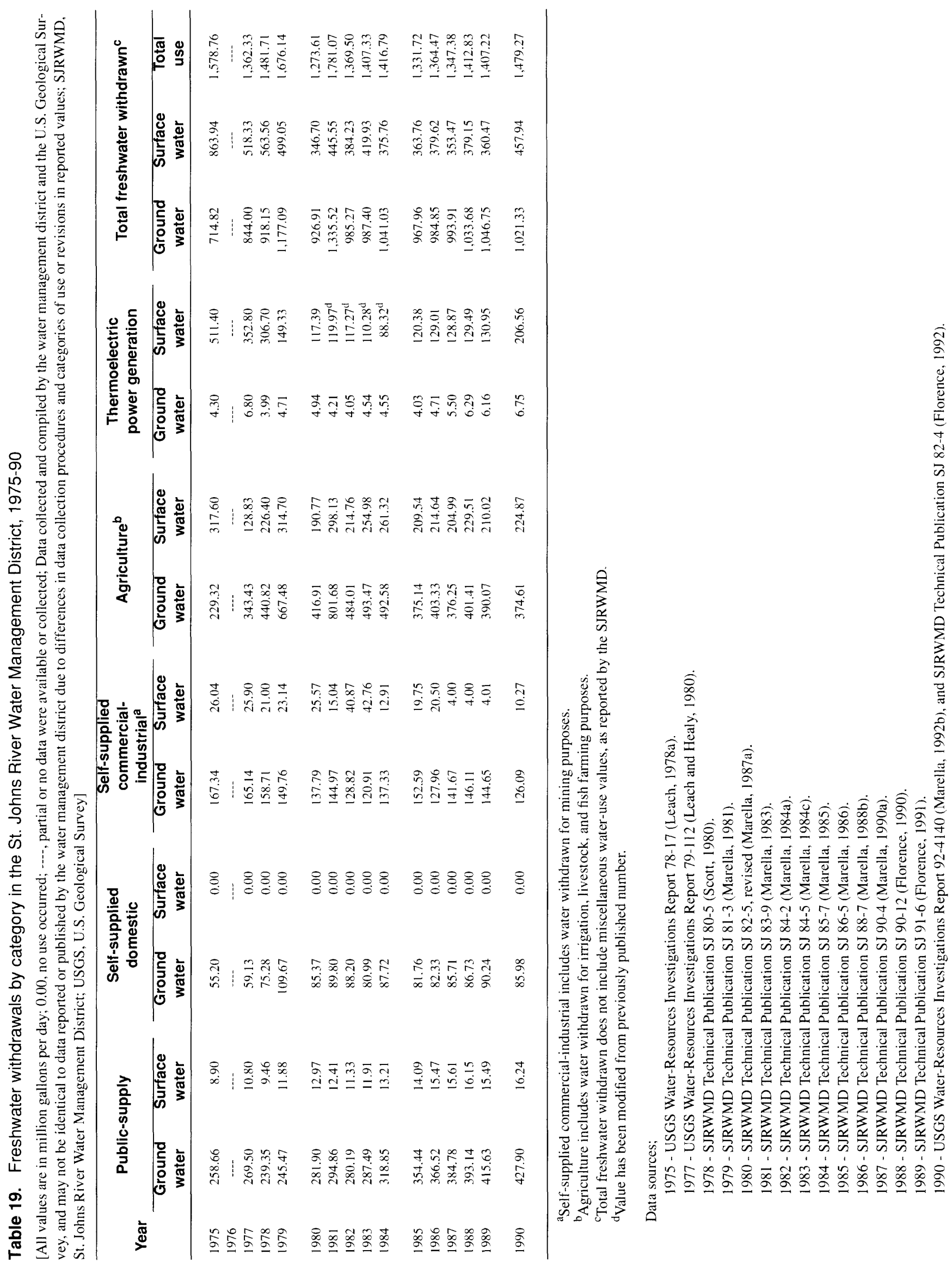


The South Florida Water Management District

The South Florida Water Management District is located in the extreme southeast and southwest area of Florida, and encompasses approximately 29 percent $(17,000$ square miles) of the total land area of the State (Fernald and Patton, 1984, p. 139). Located within the district are all or part of the following 16 counties; Broward, Charlotte (part), Collier, Dade, Glades, Hendry, Highlands (part), Lee, Martin, Monroe, Okeechobee (part), Orange (part), Osceola (part), Palm Beach, Polk (part), and St. Lucie (fig. 12 and table 17). In 1990, the district had an estimated population of nearly 5.20 million residents (Marella, 1992b, p. 29), or approximately 40 percent of the total resident population of Florida. This represents a 47 percent increase in population from the 3.54 million in 1975 (Leach, 1978a, p. 48). Total freshwater withdrawals within the district increased about 37 percent between 1975 and 1990. Specifically, fresh ground-water withdrawals increased 60 percent from $1,159 \mathrm{Mgal} / \mathrm{d}$ in 1975 to $1,851 \mathrm{Mgal} / \mathrm{d}$ in 1990 (fig. 16 and table 20). This increase primarily is due to an increase in public-supply withdrawals that is related to the population growth and the high seasonal population between 1975 and 1990. More than 95 percent of the public-supply water for the South Florida Water Management District was obtained from ground-water sources during 1990. Additional increases in ground-water withdrawals within the district occurred from an increase in acreage irrigated for agriculture. Fresh surface-water withdrawals increased by 17 percent from $1,373 \mathrm{Mgal} / \mathrm{d}$ in 1975 to $1,610 \mathrm{Mgal} / \mathrm{d}$ in 1990 (fig. 16 and table 20). Most of this increase was a result of increases in agricultural acreage, specifically citrus and sugarcane (fig. 10). Nearly 44 percent of the citrus acreage and 100 percent of the sugarcane acreage for the State was located within the South Florida Water Management District in 1990 . Nearly 50 percent of the water withdrawn in the water management district for citrus irrigation and 100 percent of the water withdrawn for sugarcane irrigation was from surface-water sources (Marella, 1992b, p. 29). The South Florida Water Management District accounted for 46 percent of the State's total freshwater use in 1990, compared to 38 percent in 1975.

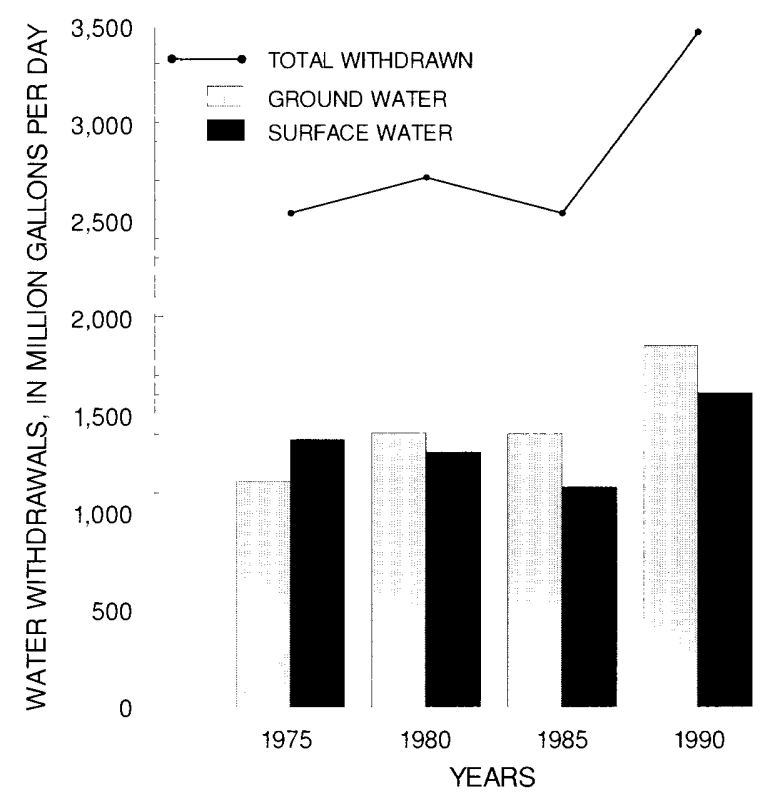

Figure 16. Freshwater withdrawn in the South Florida Water Management District by source, 1975, 1980, 1985 and 1990 (modified from Leach, 1978a, and Marella, 1986, 1987a, 1992b). 


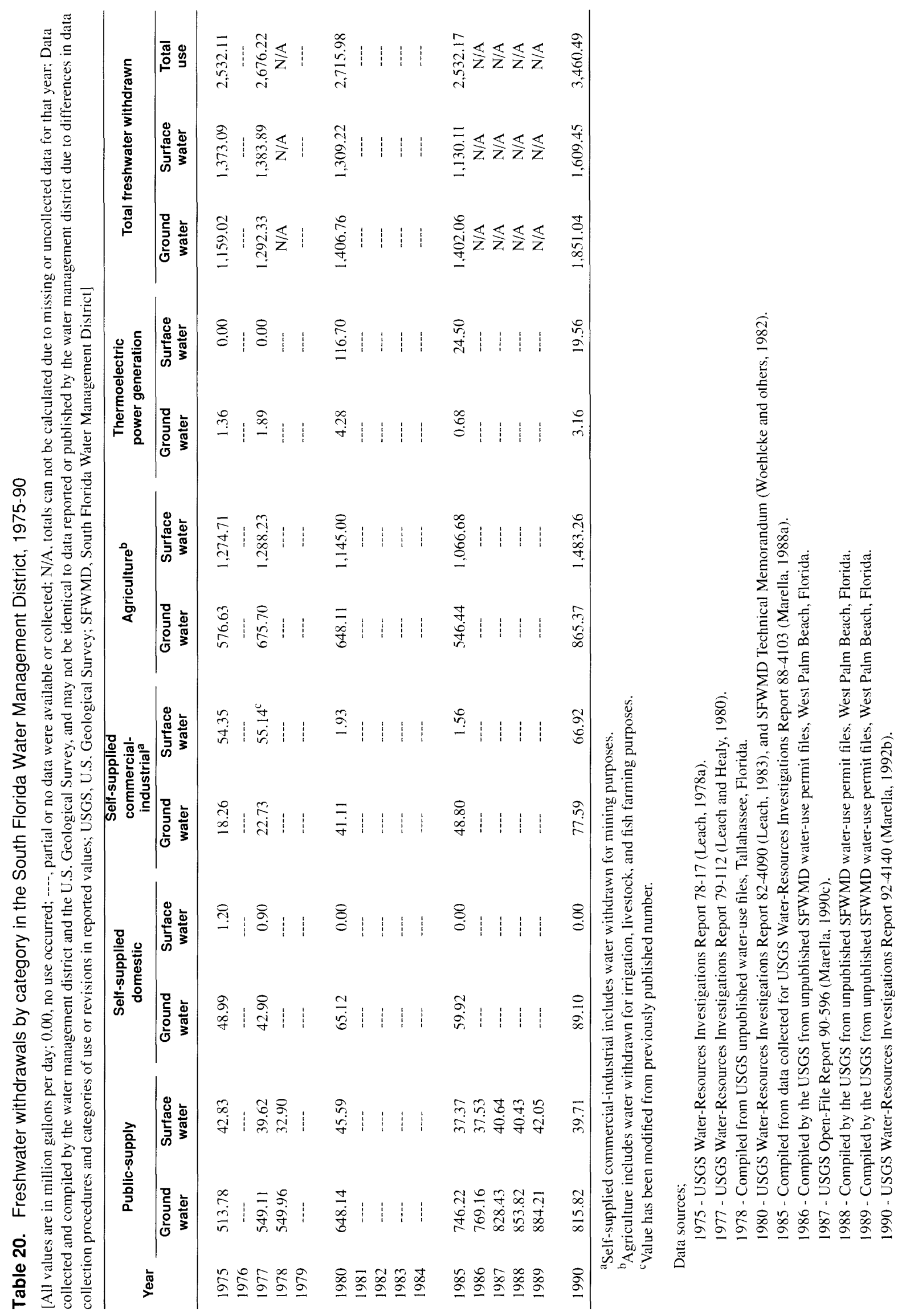




\section{The Southwest Florida Water Management District}

The Southwest Florida Water Management District is located in the south central and southwest area of Florida, and encompasses approximately 17 percent $(10,000$ square miles $)$ of the total land area of the State (Fernald and Patton, 1984, p. 178).

Located within the district are all or part of the following 16 counties; Charlotte (part), Citrus, De Soto, Hardee, Hernando, Highlands (part), Hillsborough, Lake (part), Levy (part), Manatee, Marion (part), Pasco, Pinellas, Polk (part), Sarasota, and Sumter (fig. 12 and table 17). In 1990, the district had an estimated population of nearly 3.35 million residents (Marella, 1992b, p. 29), or approximately 26 percent of the total resident population of Florida. This represents a 56 percent increase in population from the 2.15 million in 1975 (Leach, 1978a, p. 49). Total freshwater withdrawals within the district decreased about 1 percent between 1975 and 1990. However, fresh ground-water withdrawals increased 24 percent from $1,050 \mathrm{Mgal} / \mathrm{d}$ in 1975 to $1,302 \mathrm{Mgal} / \mathrm{d}$ in 1990 (fig. 17 and table 21). This increase primarily is due to an increase in public-supply withdrawals that is related to the population growth and the high seasonal population between 1975 and 1990. Nearly 72 percent of the public-supply water for the Southwest Florida Water Management District was obtained from ground-water sources during 1990. Additional increases in ground-water withdrawals within the district occurred from an increase in acreage irrigated for agriculture. Fresh surface-water withdrawals decreased nearly 50 percent from $543 \mathrm{Mgal} / \mathrm{d}$ in 1975 to $269 \mathrm{Mgal} / \mathrm{d}$ in 1990 (fig. 17 and table 21). Almost all of this decrease in fresh surface-water withdrawals between 1975 and 1990 was a result of lower usage for thermoelectric power generation. The Southwest Florida Water Management District accounted for 21 percent of the State's total freshwater use in 1990, compared to 24 percent in 1975.

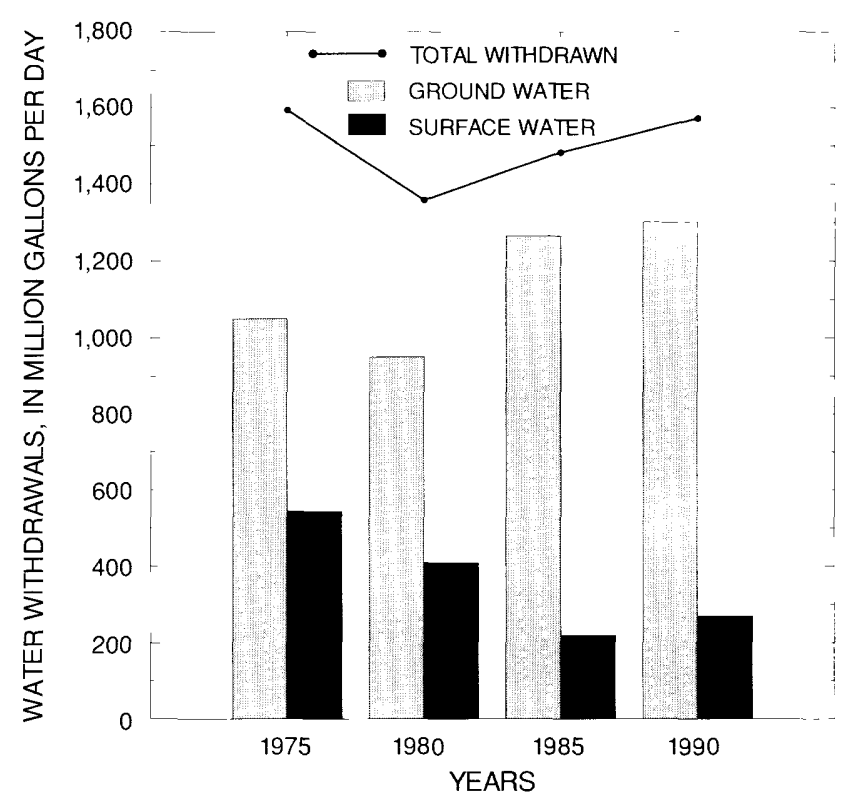

Figure 17. Freshwater withdrawn in the Southwest Florida Water Management District by source, 1975, 1980, 1985 and 1990 (modified from Leach, 1978a, Marella, 1992b, and Stiegliz, 1986). 


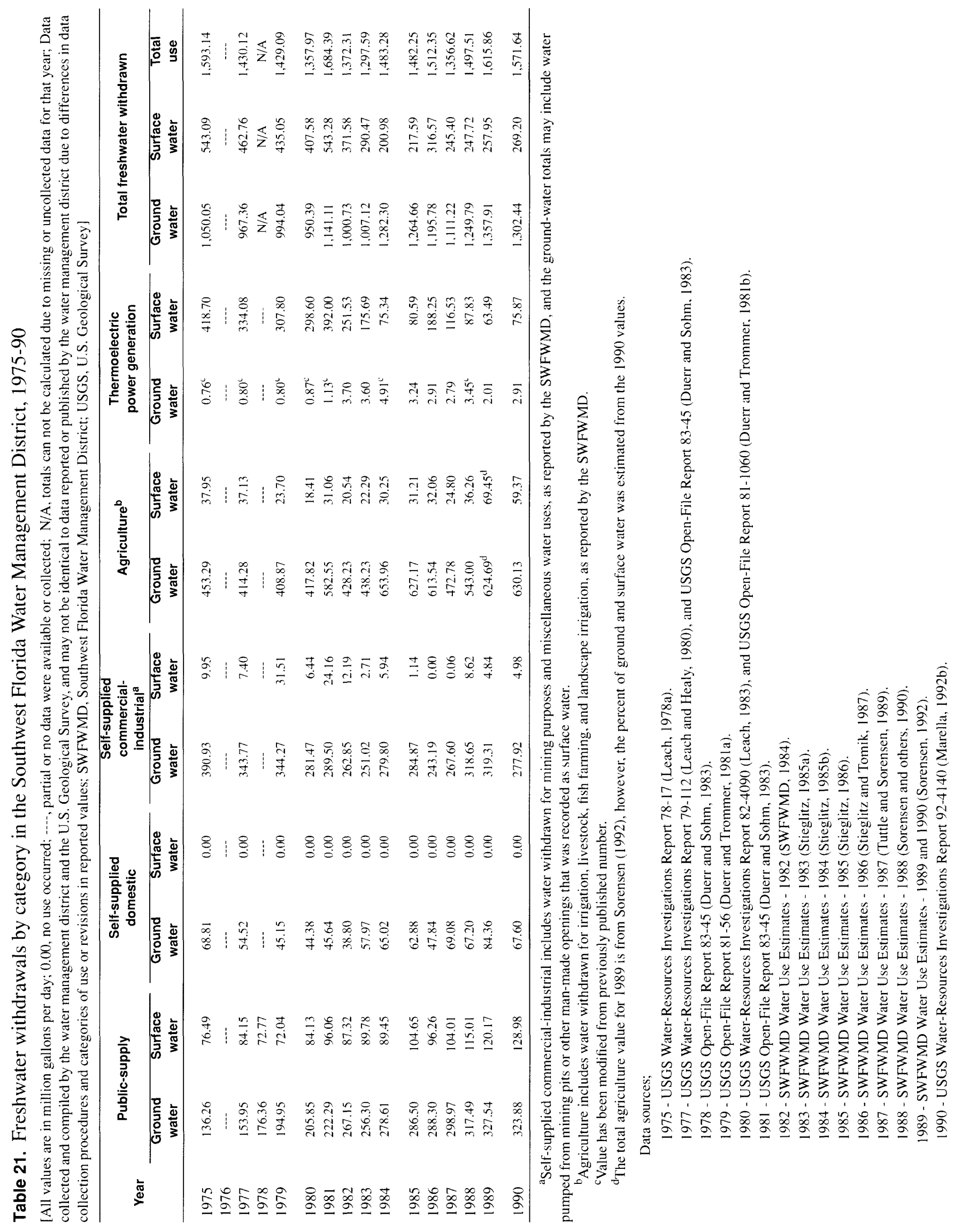




\section{The Suwannee River Water Management District}

The Suwannee River Water Management District is located in the north central area of Florida, and encompasses approximately 14 percent $(8,100$ square miles) of the total land area of the State (Fernald and Patton, 1984, p. 218). Located within the district are all or part of the following 15 counties; Alachua (part), Baker (part), Bradford (part), Columbia, Dixie, Gilchrist, Hamilton, Jefferson (part), Lafayette, Levy (part), Madison, Putnam (part), Suwannee, Taylor, and Union (fig. 12 and table 17). In 1990, the district had an estimated population of nearly 0.22 million residents (Marella, 1992b, p. 29), or approximately 1.5 percent of the total resident population of Florida. This represents nearly a 30 percent increase in population from the 0.17 million in 1975 (Leach, 1978a, p. 47). Total freshwater withdrawals within the district increased about 8 percent between 1975 and 1990. Specifically, fresh ground-water withdrawals increased 67 percent from $131 \mathrm{Mgal} / \mathrm{d}$ in 1975 to $219 \mathrm{Mgal} / \mathrm{d}$ in 1990 (fig. 18 and table 22). Increases in ground-water withdrawals within the district occurred from an increase in acreage irrigated for agriculture. Additionally, increases in ground-water withdrawals occurred from an increase in public-supply

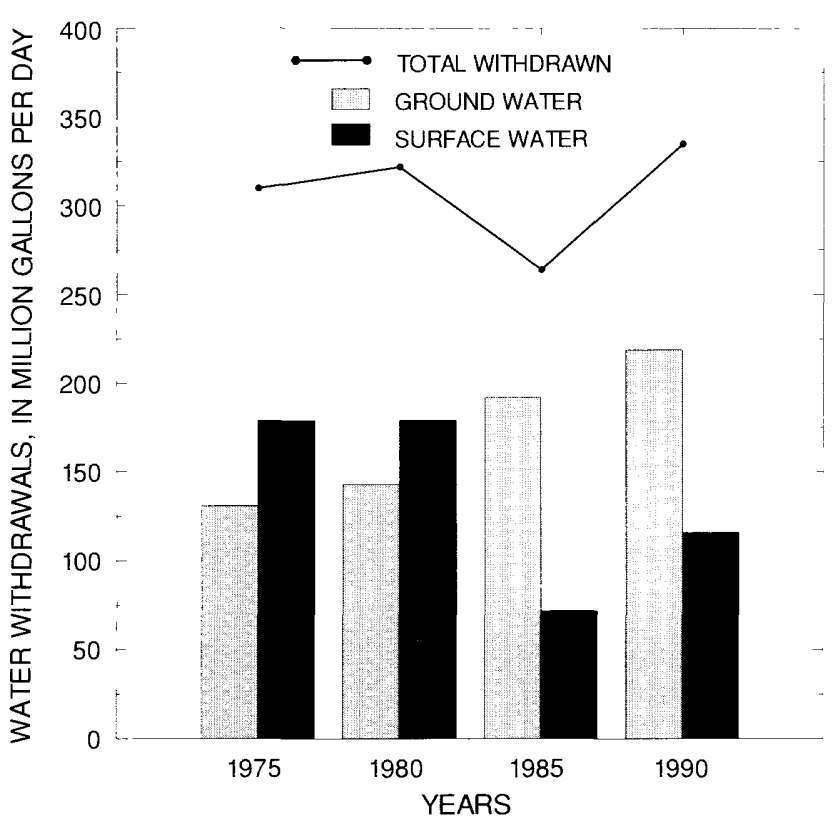

Figure 18. Freshwater withdrawn in the Suwannee River Water Management District by source, 1975, 1980, 1985 and 1990 (modified from Leach, 1978a, and Marella, 1992b). and self-supplied domestic withdrawals, as 100 percent of the water used for these categories in the district is from ground-water sources. Fresh surface-water withdrawals decreased 35 percent from $179 \mathrm{Mgal} / \mathrm{d}$ in 1975 to $116 \mathrm{Mgal} / \mathrm{d}$ in 1990 (fig. 18 and table 22). Nearly all of the decrease in fresh surfacewater withdrawals between 1975 and 1990 was a result of lower usage for thermoelectric power generation within the district. The Suwannee River Water Management District accounted for 4 percent of the State's total freshwater use in 1990 , compared to 5 percent in 1975 .

\section{SUMMARY}

The population of Florida in 1990 was estimated at 12.94 million, an increase of nearly 370 percent ( 10.17 million) from the 1950 population of 2.77 million in 1950 , and is projected to surpass 20 million by the year 2020 . In addition to population as a key water-use factor, an estimated 41 million visitors came to Florida during 1990 and the State's agricultural production was among the top 10 States in the nation. Water use (fresh and saline) in Florida increased more than 510 percent between 1950 and 1990. Through the cooperation of the Florida Department of Environmental Protection, water-use data for Florida for the period between 1950 and 1990 have been consolidated into one publication. This report aggregates and summarizes the quantities of water withdrawn (published or unpublished) for all wateruse categories (public supply, self-supplied domestic, self-supplied commercial-industrial, agriculture, and thermoelectric power generation), counties, and water management districts between 1950 and 1990. Withdrawal data are presented for ground and surface water, as well as for fresh and saline sources.

Water-use data for the early years of 1950 to 1965 were collected intermittently the USGS or the Florida Bureau of Geology. Data were generally obtained from utility operators, plant managers, and farmers by field technicians usually involved in other types of data collection (such as water levels or stream flows) or obtained by staff engineers and hydrologists conducting studies in a specific area for a certain time frame. By 1970, water use in Florida had increased to such an extent that the USGS and the Florida Bureau of Geology initiated a more formalized joint effort to collect and compile water-use data for the State. During the mid- to late-1970's, water-use data were being 


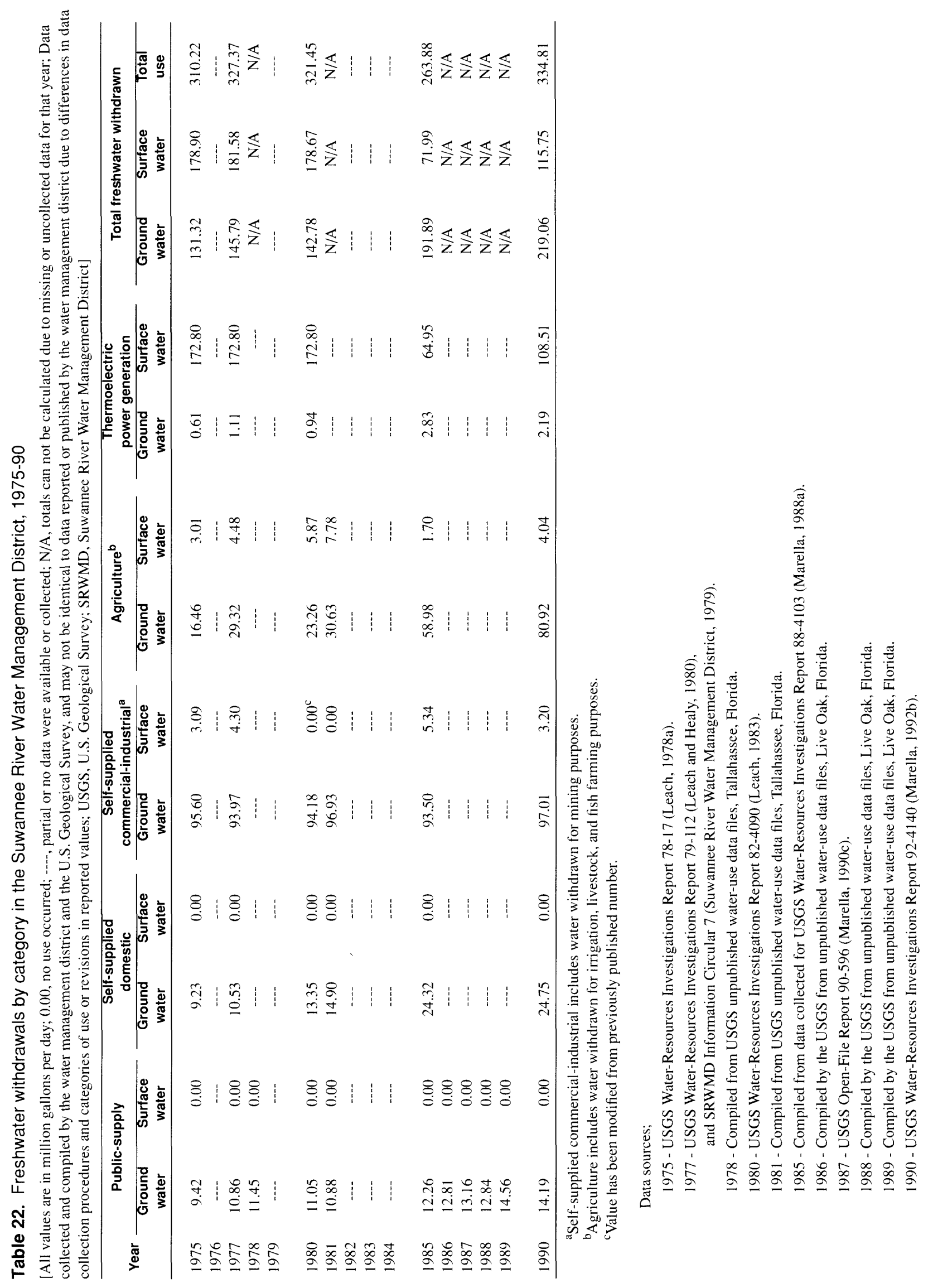


collected and compiled by the five water management districts and the USGS on a more regular basis.

During the 1980 's, each water management district was collecting water- use data based upon their individual needs. By the late 1980's, the Florida Department of Environmental Protection recognized the need to resume the compilation of water-use data on a consistent basis for Florida, and through a cooperative program with the USGS, initiated a program with the five water management districts to collect and compile these data for Florida on a five year basis.

Total water withdrawn in Florida increased 510 percent from 2,923 Mgal/d in 1950 to 17,898 $\mathrm{Mgal} / \mathrm{d}$ in 1990. Surface-water withdrawals in 1950 totaled 2,333 Mgal/d but were not differentiated between fresh and saline, therefore, comparisons between fresh and saline water were made beginning in 1955 instead of 1950. Freshwater withdrawals have increased 245 percent and saline water withdrawals increased more than 1,500 percent between 1955 and 1990. In 1990, nearly 62 percent of the freshwater withdrawn was from ground-water sources, while 38 percent was from surface-water sources. In 1955, more than 47 percent was withdrawn from groundwater sources and 53 percent was withdrawn from surface-water sources.

Water withdrawn for public supply in Florida increased 1,030 percent between 1950 and 1990. From 1950 to 1990, ground-water withdrawals for public supply increased more than 1,110 percent and surface-water withdrawals increased 650 percent. The population served by public-supply water in Florida increased from 1.66 million in 1950 to 11.23 million in 1990, and the percentage of the population served by public supply increased from 60 percent in 1950 to nearly 88 percent in 1990 . Water withdrawn for selfsupplied domestic use in Florida increased 1,110 percent from 1950 to 1990 . In 1990, an estimated 1.71 million people in Florida were selfsupplied or obtained water from small utility systems, compared to 1.11 million in 1950 . It is assumed that water withdrawn for self-supplied domestic use in Florida is derived solely from ground water, primarily because this source can provide the quantity and quality of water needed for drinking purposes.

Freshwater withdrawn for self-supplied commercial-industrial uses in Florida increased 169 percent between 1950 and 1990. From 1950 to 1990, groundwater withdrawals for self-supplied commercial-industrial use increased 125 percent and surface-water with- drawals increased nearly 2,220. Freshwater withdrawn for agricultural (irrigation and nonirrigation) use in Florida increased 915 percent between 1950 and 1990. From 1950 and 1990, ground-water withdrawals for agriculture increased 1,320 percent and surface-water withdrawals increased 670 percent. The number of acres irrigated in Florida totaled nearly 2.15 million for 1990 , compared to 0.70 million in 1960 . Freshwater withdrawals for thermoelectric power generation decreased 8 percent and saline water withdrawals increased 1,540 percent between 1955 and 1990. Overall, the total water withdrawn for thermoelectric power generation increased 672 percent between 1955 and 1990.

Since 1965, water-use data have been delineated and reported for all 67 counties in Florida. Between 1965 and 1990, total freshwater withdrawals increased in 58 of 67 counties in Florida. The largest percent increase between 1965 and 1990 occurred in Gilchrist County (1,343 percent) while the largest decrease occurred in Levy County (45 percent). Fresh groundwater was withdrawn in all 67 counties in 1965 through 1990, and increased in 65 counties between 1965 and 1990. Fresh surface-water was withdrawn in 60 counties in 1965 and 1990, and increased in 42 counties between 1965 and 1990 .

Total freshwater withdrawals within the Northwest Florida Water Management District increased about 3 percent between 1975 and 1990. Fresh ground-water withdrawals increased 58 percent while fresh surface-water withdrawals decreased 16 percent between 1975 and 1990. The population of the Northwest Florida Water Management District increased 30 percent from 0.77 million in 1975 to 1.01 million in 1990. Total freshwater withdrawals within the St. Johns River Water Management District decreased about 6 percent between 1975 and 1990. Fresh ground-water withdrawals increased 43 percent while fresh surface-water withdrawals decreased 47 percent between 1975 and 1990. The population of the St. Johns River Water Management District increased 54 percent from 2.06 million in 1975 to 3.17 million in 1990. Total freshwater withdrawals within the South Florida Water Management District increased about 37 percent between 1975 and 1990. Fresh groundwater withdrawals increased 60 percent while fresh surface-water withdrawals increased 17 percent between 1975 and 1990. The population of the South Florida Water Management District increased 47 percent from 3.54 million in 1975 to 5.20 million in 1990. Total freshwater withdrawals within the Southwest 
Florida Water Management District decreased about 1 percent between 1975 and 1990. Fresh ground-water withdrawals increased 24 percent while fresh surfacewater withdrawals decreased 50 percent between 1975 and 1990. The population of the Southwest Florida Water Management District increased 56 percent from 2.15 million in 1975 to 3.35 million in 1990 . Total freshwater withdrawals within the Suwannee River Water Management District increased about 8 percent between 1975 and 1990. Fresh ground-water withdrawals increased 67 percent while fresh surfacewater withdrawals decreased 35 percent between 1975 and 1990. The population of the Suwannee River Water Management District increased 30 percent from 0.17 million in 1975 to 0.22 million in 1990 .

\section{SELECTED REFERENCES}

Bielby, Camille, 1987, 1985 Annual water use survey: Havana, Northwest Florida Water Management District Program Development Series 87-1, 107 p.

Bucca, Jane, and Marella, R.L., 1992, An improved method for determining the nonresidential water-use component of total public water-supply estimates, in Jones, M.E., and Laenen, Antonius, ed., Interdisciplinary approaches in hydrology and hydrogeology, American Institute of Hydrology, proceedings from 1992 meeting in Portland, Oregon, p. $511-523$

Buros, O.K., 1989, Desalting practices in the United States: American Water Works Association Journal, v. 81, no. 11, November, p. 38-42.

Campbell, K.M., 1986, The industrial minerals of Florida: Tallahassee, Florida Bureau of Geology Information Circular No. 102, 94 p.

Conover, C.S., Vecchioli, John, and Foose, D.W., 1989, Ground-water sources and withdrawals for 1985: Tallahassee, Florida Bureau of Geology Map Series 124, 1 sheet.

Dietrich, T.S., 1978, The urbanization of Florida's population: An historical perspective of county growth 1830-1970: Gainesville, University of Florida, Bureau of Economic and Business Research, 211 p.

Duerr, A.D., and Sohm, J.E., 1983, Estimated water use in Southwest Florida, 1981, and summary of annual water use, 1970, 1975, and 1977-81: U.S. Geological Survey Open-File Report 83-45, 75 p.

Duerr, A.D., and Trommer, J.T., 1981 a, Estimated water use in the Southwest Florida Water Management District and adjacent areas, 1979: U.S. Geological Survey Open-File Report 81-56, 58 p.
, 1981b, Estimated water use in the Southwest Florida Water Management District and adjacent areas, 1980: U.S. Geological Survey Open-File Report 811060, $60 \mathrm{p}$.

__ 1982, The benchmark farm program-a method for estimated irrigation water use in Southwest Florida: U.S. Geological Survey Water-Resources Investigations Report 82-17, 49 p.

Dykes, G.M., and Conlon, W.J., 1989, Use of membrane technology in Florida: American Water Works Association Journal, v. 81, no. 11, November, p. 43-46.

Fernald, E.A., and Patton, D.J., 1984, Water resources atlas of Florida: Tallahassee, Florida State University, 291 p.

Florence, B.L., 1990, Annual water use survey: 1988: Palatka, St. Johns River Water Management District Technical Publication SJ 90-12, 128 p.

, 1991, Annual water use survey: 1989: Palatka, St. Johns River Water Management District Technical Publication SJ 91-6, 126 p.

, 1992, Annual water use survey: 1990: Palatka, St. Johns River Water Management District Technical Publication SJ 92-4, 122 p.

Florida Agricultural Statistics Service, 1978, Field crop summary 1977: Orlando, Florida Department of Agriculture and Consumer Services, $35 \mathrm{p}$.

__ 1991, Field crop summary 1990: Orlando, Florida Department of Agriculture and Consumer Services, $19 \mathrm{p}$.

$\longrightarrow$, 1992, Commercial citrus inventory 1992: Orlando, Florida Department of Agriculture and Consumer Services, $109 \mathrm{p}$.

Florida Department of Environmental Regulation, 1990, Drinking water standards, monitoring, and reporting: Tallahassee, Chap. 17-550, 48 p.

Florida Division of Tourism, 1990, Florida visitor study, 1990: Executive summary: Tallahassee, Florida Department of Commerce, Office of Tourism Research, 75 p.

Florida Water Resources Study Commission, 1956, Florida's water resources: Gainesville, Report to the Governor of Florida and the 1957 Legislature, 94 p.

Franks, B.J., 1981, Land application of domestic wastewater in Florida-Statewide assessment of impact on ground-water quality: U.S. Geological Survey Water-Resources Investigations 81-3, 37 p.

Healy, H.G., 1972, Public water supplies of selected municipalities in Florida, 1970: Tallahassee, Florida Bureau of Geology Information Circular no. 81, 213 p. , 1977, Public water supplies of selected municipalities in Florida, 1975: U.S. Geological Survey Water-Resources Investigations Report 77-53, $309 \mathrm{p}$. 
Holland, T.W., 1992, Water-use data collection techniques in the southeastern United States, Puerto Rico, and the U.S. Virgin Islands: U.S. Geological Survey WaterResources Investigations Report 92-4028, 76 p.

Hughes, G.H., 1975, Perspective on use of fresh water for cooling systems of thermoelectric powerplants in Florida: U.S. Geological Survey Water-Resources Investigations $43-75,30 \mathrm{p}$.

Kranzer, B.S., 1983, Water use in the Northwest Florida Water Management District, an examination of current and past use: Havana, Northwest Florida Water Management District Water Resources Special Report 83-3, $47 \mathrm{p}$.

Leach, S.D., 1978a, Source, use, and disposition of water in Florida, 1975: U.S. Geological Survey WaterResources Investigations 78-17, 90 p.

-1978 b, Freshwater use in Florida, 1975: Tallahassee, Florida Bureau of Geology Map Series 87,1 sheet.

- , 1982, Estimated water use in Florida, 1980: Tallahassee, Florida Bureau of Geology Map Series 103, 1 sheet.

,- 1982 , Consumptive use of freshwater in Florida, 1980: Tallahassee, Florida Bureau of Geology Map Series 105, 1 sheet.

Leach, S.D.,, 1983, Source, use, and disposition of water in Florida, 1980: U.S. Geological Survey WaterResources Investigations 82-4090, 337 p.

-1984, Projected public supply and rural (selfsupplied) water use in Florida through the year 2020: Tallahassee, Florida Bureau of Geology Map Series 108,1 sheet.

Leach, S.D., and Healy, H.G., 1980, Estimated water use in Florida, 1977, U.S. Geological Survey WaterResources Investigations Report 79-112, 76 p.

Lewis, K.C., Carriker, Roy, and Marella, R.L., 1981, Analysis of residential demand for water in the St. Johns River Water Management District: Palatka, St. Johns River Water Management District Technical Publication SJ 81-2, 107 p.

MacKichan, K.A., 1951, Estimated use of water in the United States, 1950: U.S. Geological Survey Circular $115,13 \mathrm{p}$.

, 1957, Estimated use of water in the United States, 1955, U.S. Geological Survey Circular 398, 18 p.

MacKichan, K.A., and Kammerer, J.C., 1961, Estimated use of water in the United States, 1960: U.S. Geological Survey Circular 456, 44 p.

Marella, R.L., 1981, Annual water use survey; 1979: Palatka, St. Johns River Water Management District Technical Publication SJ 81-3, 127 p.

- 1982 , Annual water use survey; 1980: Palatka, St. Johns River Water Management District Technical Publication SJ 82-5, 107 p. 1983a, Ground-water withdrawals from the Floridan Aquifer in Duval County - 1980: Palatka, St. Johns River Water Management District Technical Publication SJ 83-7, 1 sheet.

_, 1983b, Annual water use survey; 1981: Palatka,

St. Johns River Water Management District Technical Publication SJ 83-9, 107 p.

-, 1984a, Annual water use survey; 1982: Palatka,

St. Johns River Water Management District Technical Publication SJ 84-2, 97 p.

, 1984b, Ground-water withdrawals from the

Floridan Aquifer in the Nassau County Area: Palatka,

St. Johns River Water Management District Map

Series 84-4, 1 sheet.

-, 1984c, Annual water use survey; 1983: Palatka,

St. Johns River Water Management District Technical

Publication SJ 84-5, 103 p.

, 1984d, Ground-water withdrawals from the

Floridan Aquifer in the Clay and portions of Bradford

Counties: Palatka, St. Johns River Water Management

District Map Series 84-14, 1 sheet.

, 1985, Annual water use survey; 1984: Palatka,

St. Johns River Water Management District Technical

Publication SJ 85-7, 113 p.

, 1986, Annual water use survey; 1985: Palatka,

St. Johns River Water Management District Technical

Publication SJ 86-5, 117 p.

- 1987a, Annual water use survey; 1980 Revised

Edition: Palatka, St. Johns River Water Management

District Technical Publication SJ 82-5, 55 p.

, 1987b, Water use, Chapter 8: in the Indian River

Lagoon Joint Reconnaissance Report, St. Johns River

Water Management District and the South Florida

Water Management District, November 1987 ,

p. 8-1 8-9.

, 1988a, Water withdrawals, use and trends in

Florida, 1985: U.S. Geological Survey Water-

Resources Investigations Report 88-4103, 43 p. , 1988b, Water withdrawals, use and trends in the

St. Johns River Water Management District; 1986:

Palatka, St. Johns River Water Management District

Technical Publication SJ 88-7, 128 p.

, 1989, Freshwater withdrawals, water-use trends in

Florida, 1985: Tallahassee, Florida Bureau of Geology

Map Series 123, I sheet.

_, 1990a, Annual water use survey; 1987: Palatka,

St. Johns River Water Management District Technical

Publication SJ 90-4, 126 p.

, 1990b, Florida water supply and use, in the

National water summary 1987--Hydrologic events and water supply and use: U.S. Geological Survey WaterSupply Paper 2350, p. 207-214.

, 1990c, Public supply water use in Florida, 1987:

U.S. Geological Survey Open-File Report 90-596,

39 p. 
Marella, R.L., 1992a, Factors that affect public-supply water use in Florida, with a section on projected water use to the year 2020: U.S. Geological Survey WaterResources Investigations Report 91-4123, 35 p. , 1992b, Water withdrawals, use and trends in Florida, 1990: U.S. Geological Survey WaterResources Investigations Report 92-4140, 38 p. , 1993, Public supply water use in Florida, 1990: U.S. Geological Survey Open-File Report 93-134, $46 \mathrm{p}$.

$\ldots, 1994$, Estimated discharge of treated wastewater in Florida, 1990: U.S. Geological Survey Open-File Report 93-364, 53 p.

Marella, R.L., Fanning, J.L., and Mooty, W.S., 1993, Estimated use of water in the ApalachicolaChattahoochee-Flint River basin during 1990, with State summaries for 1970 to 1990: U.S. Geological Survey Water-Resources Investigations Report 934084, 45 p.

McKenzie, D.J., 1990, Water-resources potential of the freshwater lens at Key West, Florida: U.S. Geological Survey Water-Resources Investigations Report 90$4115,24 \mathrm{p}$.

McPherson, B.F., and Hammett, K.M., 1991, Tidal Rivers of Florida, in Livingston, R.J., ed., The Rivers of Florida: Springer-Verlag, Ecological Studies 83, p. $31-46$.

Meyer, F.W., 1974, Availability of ground water for the U.S. Navy well field near Florida City, Dade County, Florida: U.S. Geological Survey Open-File Report FL-74014, 50 p.

Miller, M.L., and Alvarez, J.A., 1984, Public-supply water use, Palm Beach County, Florida, 1978-82: U.S. Geological Survey Open-File Report 84-240, 14 p.

Murray, C.R., 1968, Estimated use of water in the United States, 1965: U.S. Geological Survey Circular 556, $53 \mathrm{p}$.

Murray, C.R., Reeves, E.B., 1972, Estimated use of water in the United States, 1970: U.S. Geological Survey Circular 676, $37 \mathrm{p}$.

, 1977, Estimated use of water in the United States, 1975: U.S. Geological Survey Circular 765, 37 p.

O'Donnell, T.H., 1977, Municipal water suppliers in Lee County, 1974: U.S. Geological Survey Open-File Report 77-277, 96 p.

Pride, R.W., 1973, Estimated use of water in Florida, 1970: Tallahassee, Florida Bureau of Geology Information Circular 83, 31 p.

, 1975, Estimated water use in Florida, 1965 (2d ed.): Tallahassee, Florida Bureau of Geology Map Series 36, 1 sheet.

St. Johns River Water Management District, 1984, Consumptive uses of water; applicant's handbook: Palatka, Resource Management Department, June 1, 1984, 49 p.
Schmidt, Walter, Hoenstine, R.W., Knapp, M.S., Lane, Ed., Ogden, G.M. Jr., and Scott, T.M., 1979, The limestone, dolomite and coquina resources of Florida:

Tallahassee, Florida Bureau of Geology Information Circular 83, $31 \mathrm{p}$.

Scott, Elaine, 1980, Annual water use survey; 1978: Palatka, St. Johns River Water Management District Technical Publication SJ 80-5, 107 p.

Smajstrla, A.G., 1986, Agricultural field scale irrigation requirements simulation model; Technical Manual: Gainesville, University of Florida, Agricultural Engineering Department, $52 \mathrm{p}$.

Smajstrla, A.G., Boman, B.J., Clark, G.A., Haman, D.Z., Harrison, D.S., Izuno, F.T., and Zazueta, F.S., 1988, Efficiencies of Florida agricultural irrigation systems: Gainesville, University of Florida, Institute of Food and Agricultural Sciences, Bulletin 247, 15 p.

Smith, S.K., 1991, Population growth of States and regions 1980-1990: Gainesville, University of Florida, Bureau of Economic and Business Research, Economic Leaflets, v. 50, no. 1, 4 p.

Smith, S.K., and Bayya, Ravi, 1991, Projections of Florida population by county 1990-2020: Gainesville, University of Florida, Bureau of Economic and Business Research, v. 24, no. 2, bulletin no. 96, 8 p.

Solley, W.B., Chase, E.B., and Mann, W.B., 1983, Estimated use of water in the United States in 1980: U.S. Geological Survey Circular 1001, 56 p.

Solley, W.B., Merk, C.F., and Pierce, R.R., 1988, Estimated use of water in the United States in 1985: U.S. Geological Survey Circular 1004, 82 p.

Solley, W.B., Pierce, R.R., and Perlman, H.A., 1993, Estimated use of water in the United States in 1990: U.S. Geological Survey Circular 1081, 76 p.

Sorensen, L.A., 1992, 1989 and 1990 Estimated water use in the Southwest Florida Water Management District: Brooksville, Southwest Florida Water Management District, Planning Department, 132 p.

Sorensen, L.A., Burns, L.L., Miles, D.M., Norris, Henry, and Tuttell, Maryellen, 1990, 1988 Estimated water use in the Southwest Florida Water Management District: Brooksville, Southwest Florida Water Management District, Planning Department, 96 p.

South Florida Water Management District, 1990, Desalination: An additional water source for south Florida: West Palm Beach, Office of Communications, (PIO 276290 5M), 4 p.

Southwest Florida Water Management District, 1984, Estimated water use in the Southwest Florida Water Management District, 1982: Brooksville, Southwest Florida Water Management District Planning and Performance Evaluation Section, $20 \mathrm{p}$.

Spechler, R.M., 1983, Estimated irrigation water use in Florida, 1980: Tallahassee, Florida Bureau of Geology Map Series 106, 1 sheet. 
Stieglitz, E.H., 1985a, Estimated water use in the Southwest Florida Water Management District, 1983:

Brooksville, Southwest Florida Water Management District, $33 \mathrm{p}$.

, 1985 b, Estimated water use in the Southwest

Florida Water Management District, 1984:

Brooksville, Southwest Florida Water Management

District, $44 \mathrm{p}$. , 1986, Estimated water use in the Southwest Florida Water Management District, 1985: Brooksville, Southwest Florida Water Management District, 53 p.

Stieglitz, E.H., and Tomik, K.E., 1987, Estimated water use in the Southwest Florida Water Management District, 1986: Brooksville, Southwest Florida Water Management District, $57 \mathrm{p}$.

Suwannee River Water Management District, 1979, Water use 77: Live Oak, Suwannee River Water Management District Information Circular \# 7, 47 p.

Taylor, G.F., 1993, Cocoa well field ground-water monitoring network: U.S. Geological Survey Annual Data Summary - 1992, 103 p.

Tibbals, C.H., and Frazee, J.M.,Jr., 1976, Ground-water hydrology of the Cocoa well-field area, Orange County, Florida: U.S. Geological Survey WaterResources Investigations Report 75-676, 67 p.

Tuttell, Maryellen, and Sorensen, L.A., 1989, 1987 Estimated water use in the Southwest Florida Water Management District: Brooksville, Southwest Florida Water Management District, 99 p.
U.S. Bureau of the Census, 1984, 1982 Census of Agriculture, Florida state and county data: Washington D.C., U.S. Department of Commerce, Bureau of the Census, part 9, v. 1, $380 \mathrm{p}$. , 1989, 1987 Census of Agriculture, Florida state and county data: Washington D.C., U.S. Department of Commerce, Bureau of the Census, part 9, v. 1, 390 p.

U.S. Department of Agriculture, 1991, State financial summary, 1990: Washington D.C., U.S. Department of Agriculture, Economic Research Service, 238 p.

U.S. Soil Conservation Service, 1970, Irrigation water requirement (revised), U.S. Department of Agriculture Technical Release No. 21, 88 p.

, 1982, Florida irrigation guide: Gainesville, U.S. Department of Agriculture, Soil Conservation Service, $300 \mathrm{p}$.

University of Florida, 1976, Florida estimates of population, 1975: Gainesville, University of Florida, Bureau of Economic and Business Research, 51 p. -, 1986, Florida estimates of population, 1985: Gainesville, University of Florida, Bureau of Economic and Business Research, 48 p.

- 1991, Florida population; Census summary 1990: Gainesville, University of Florida, Bureau of Economic and Business Research, $55 \mathrm{p}$.

Woehlcke, L.C., Bucca, Jane, and Loving, D.R., 1982, A potable water use data base for South Florida, 1980: West Palm Beach, South Florida Water Management District, Resource Planning Department, Technical Memorandum, $23 \mathrm{p}$. 
APPENDIX 


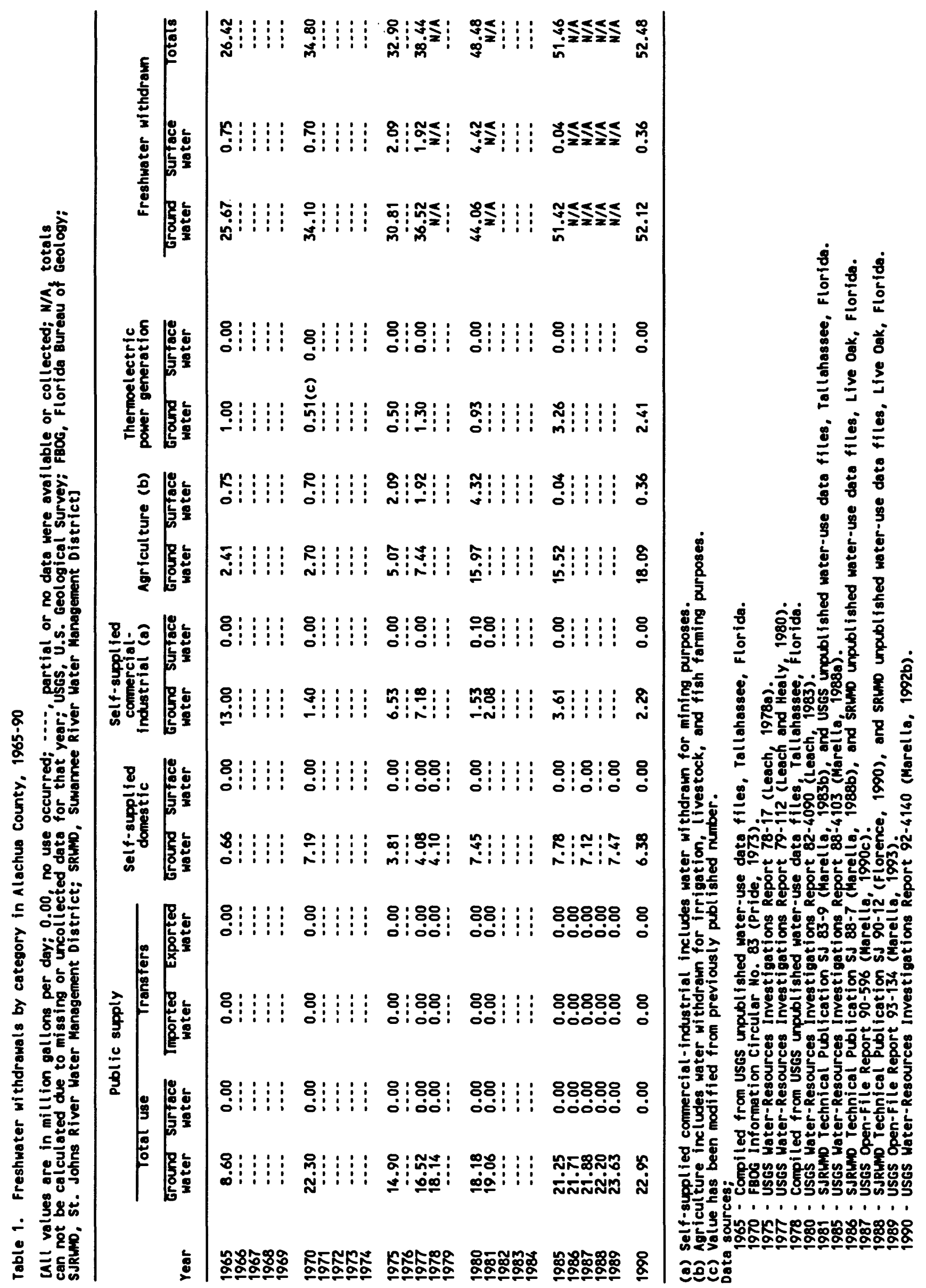




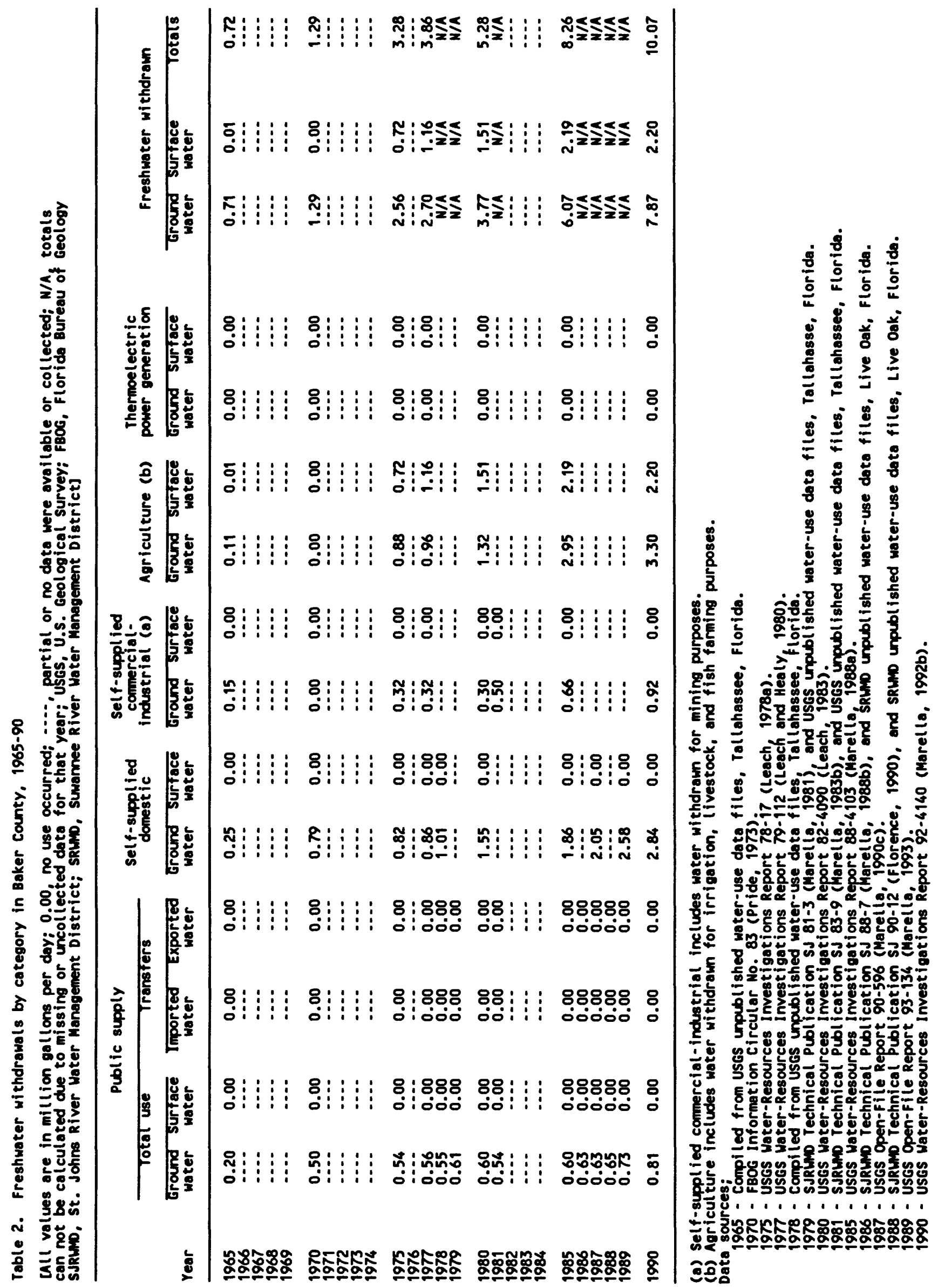




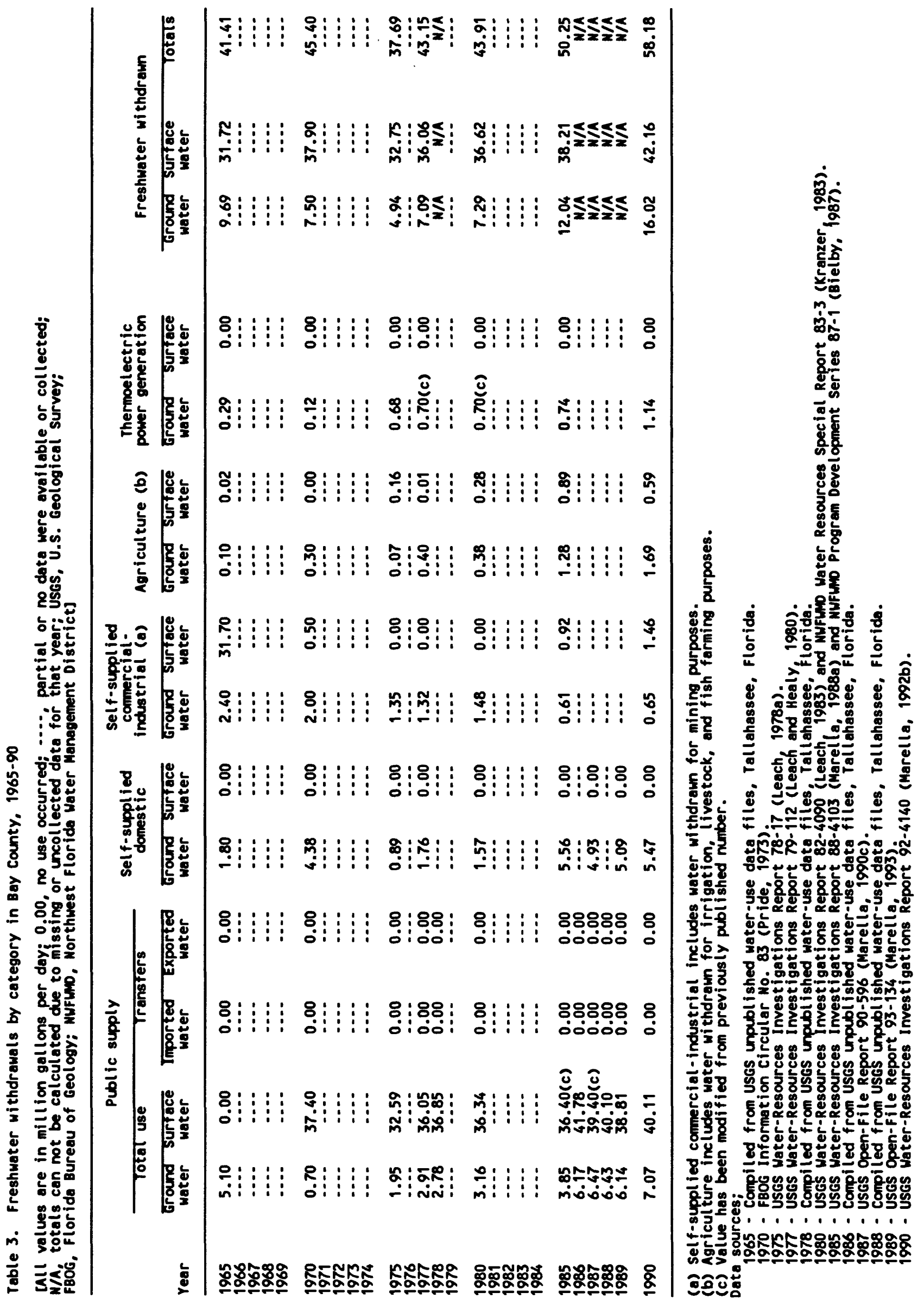




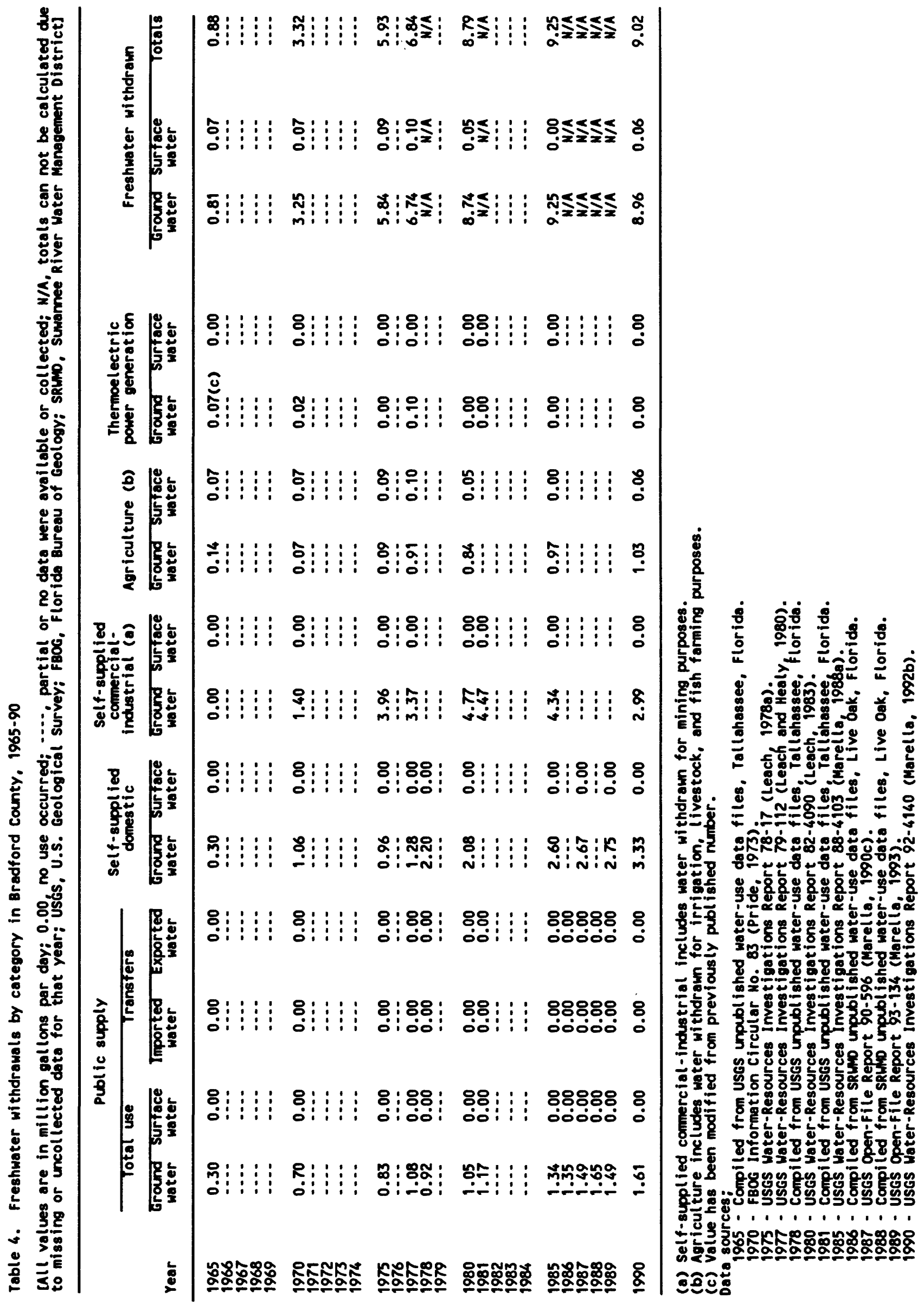




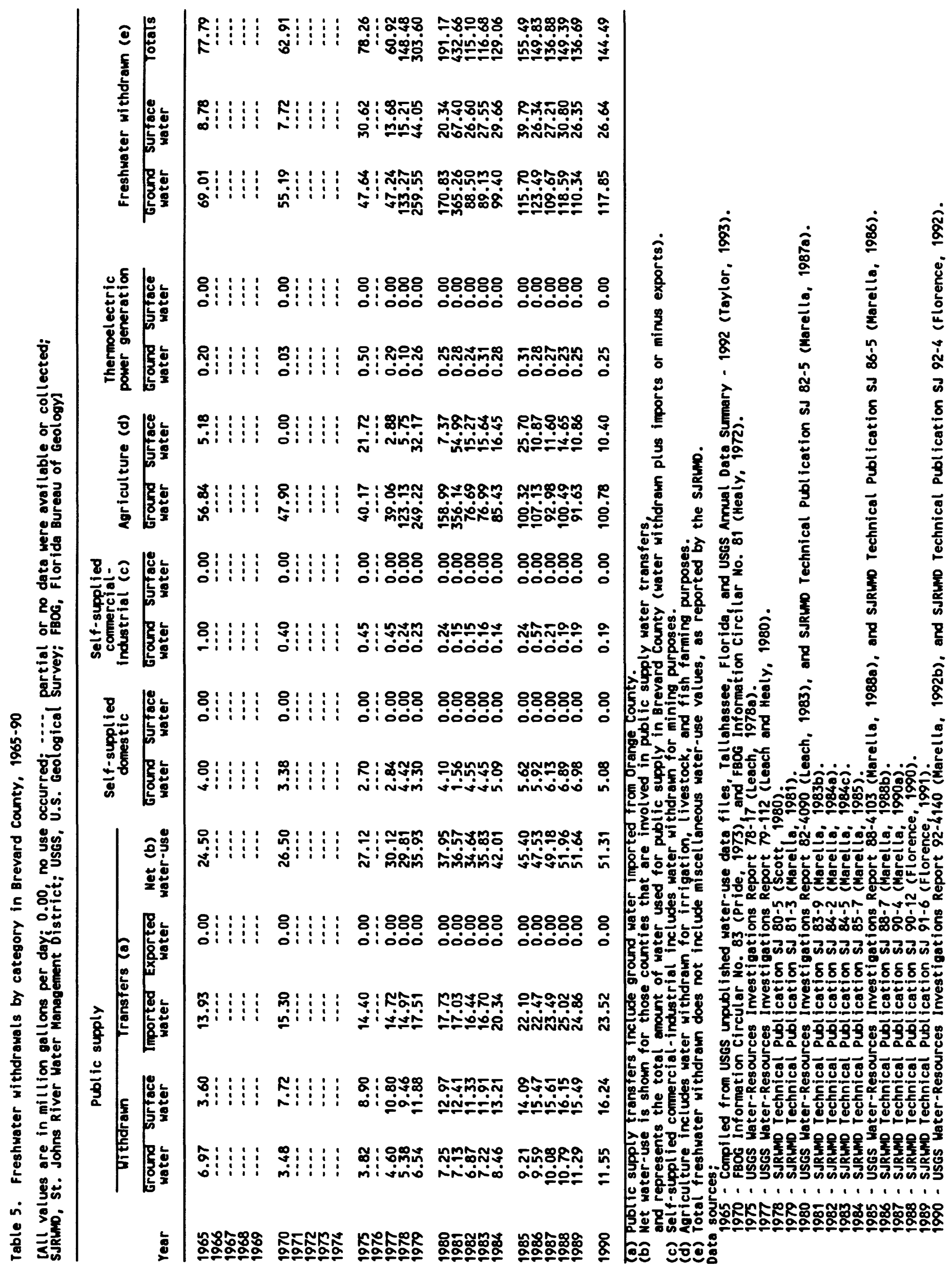




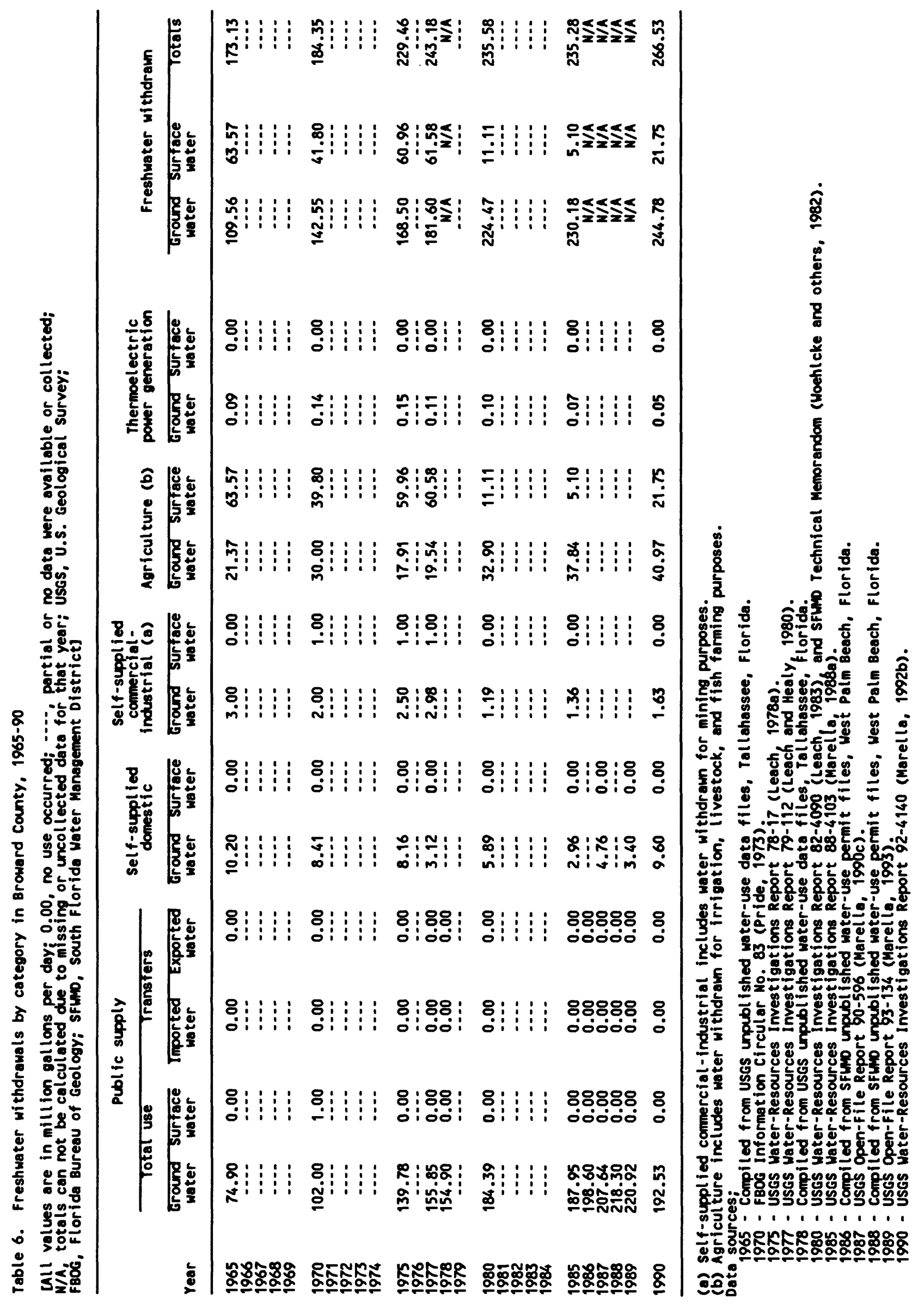




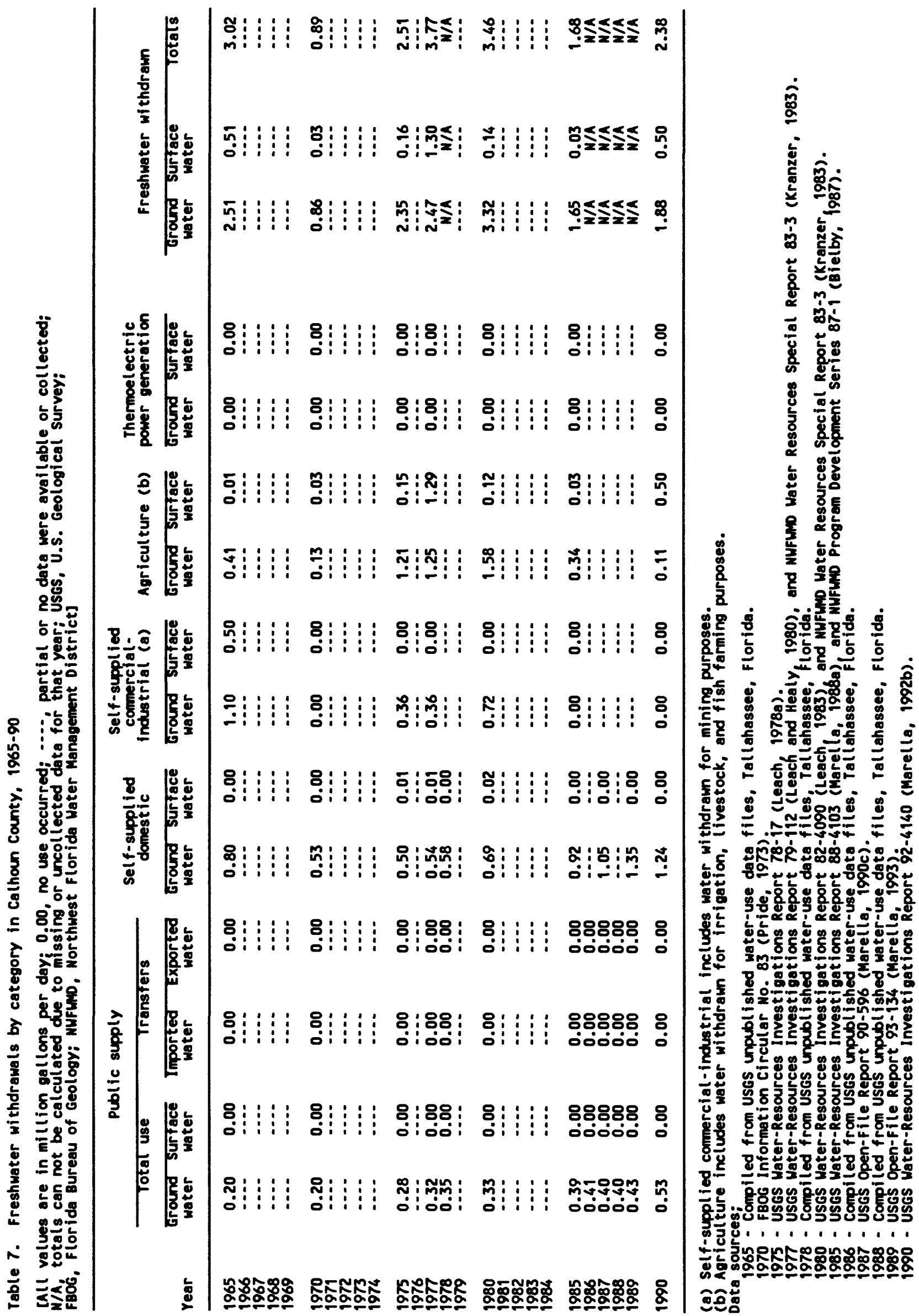




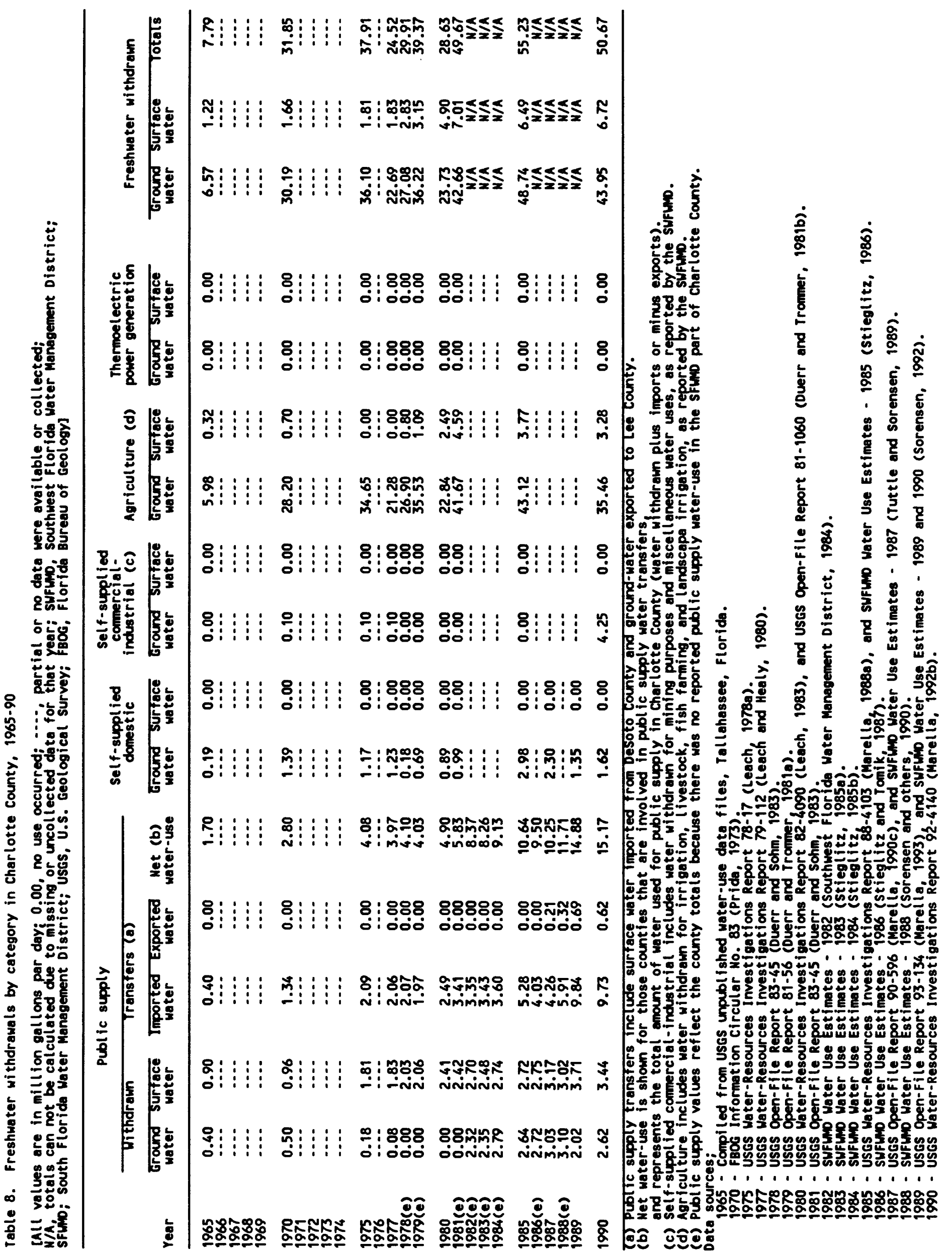




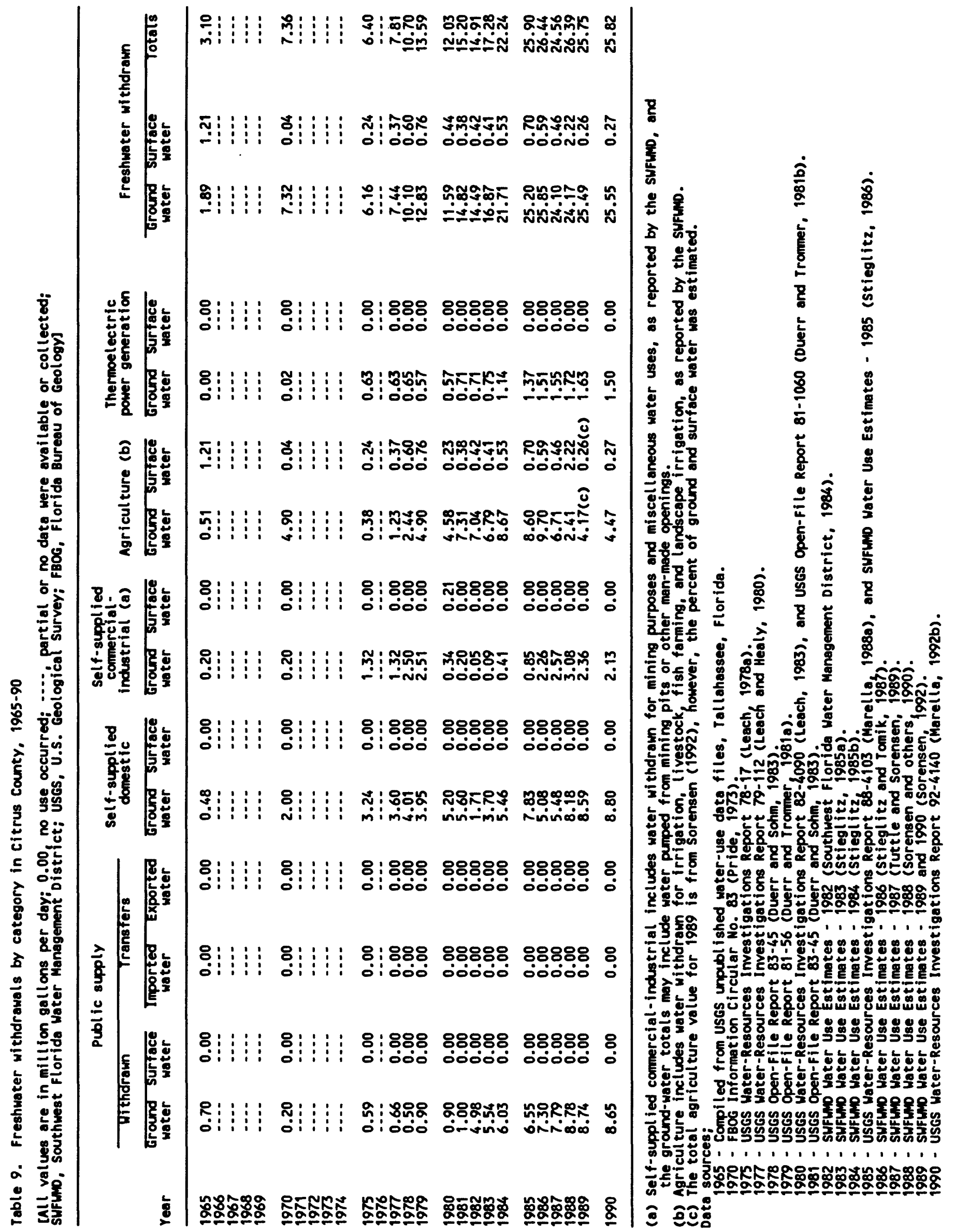




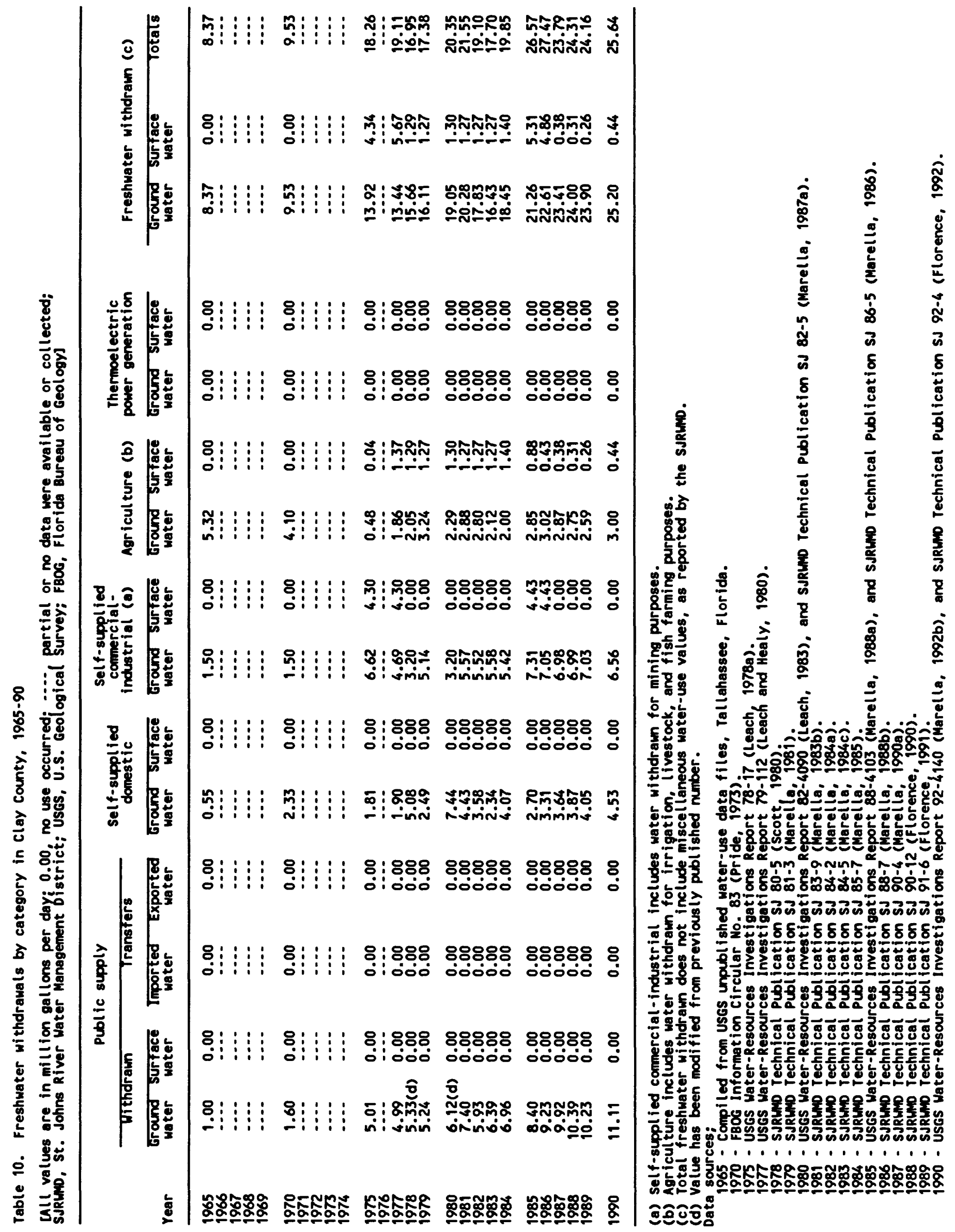




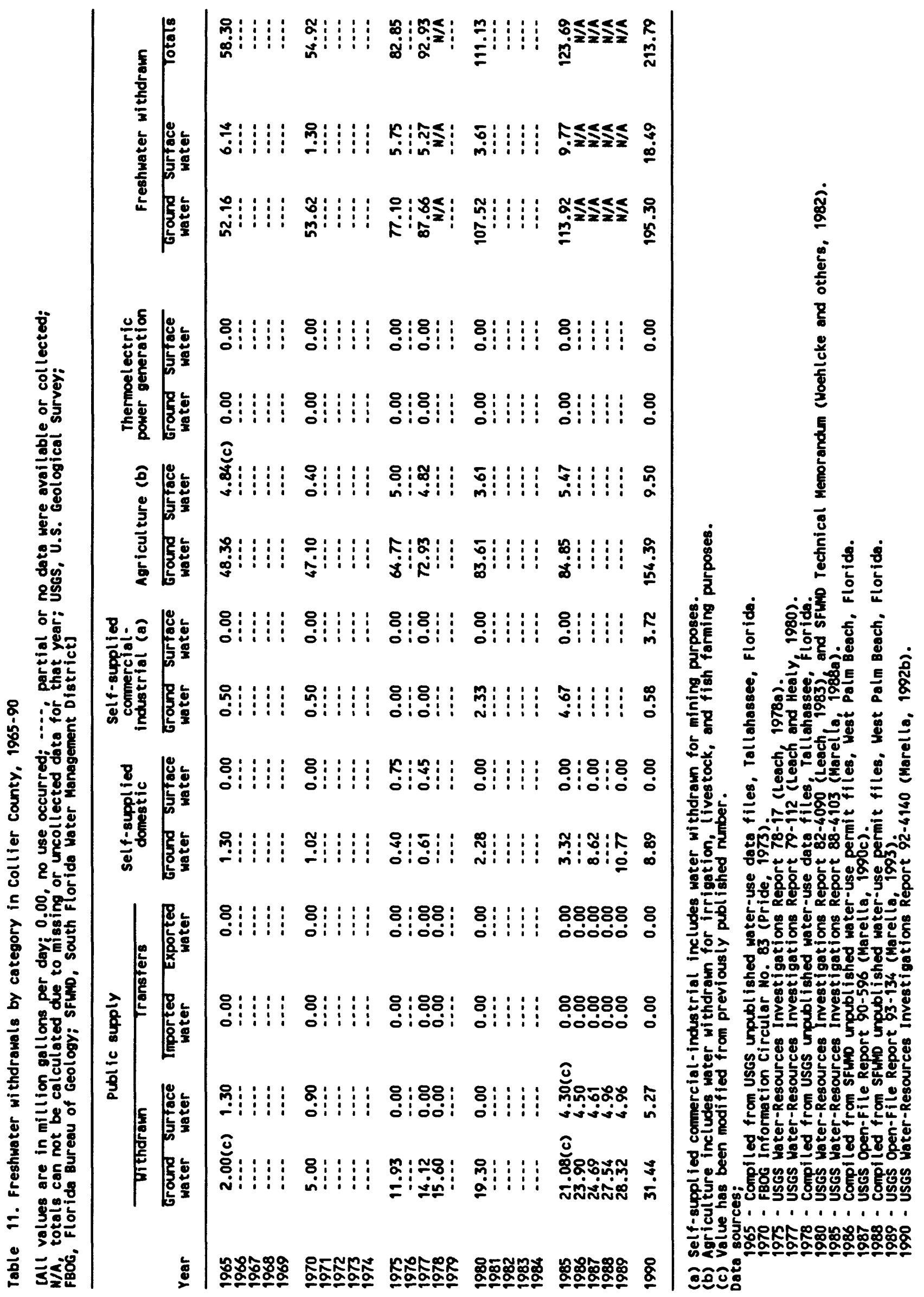




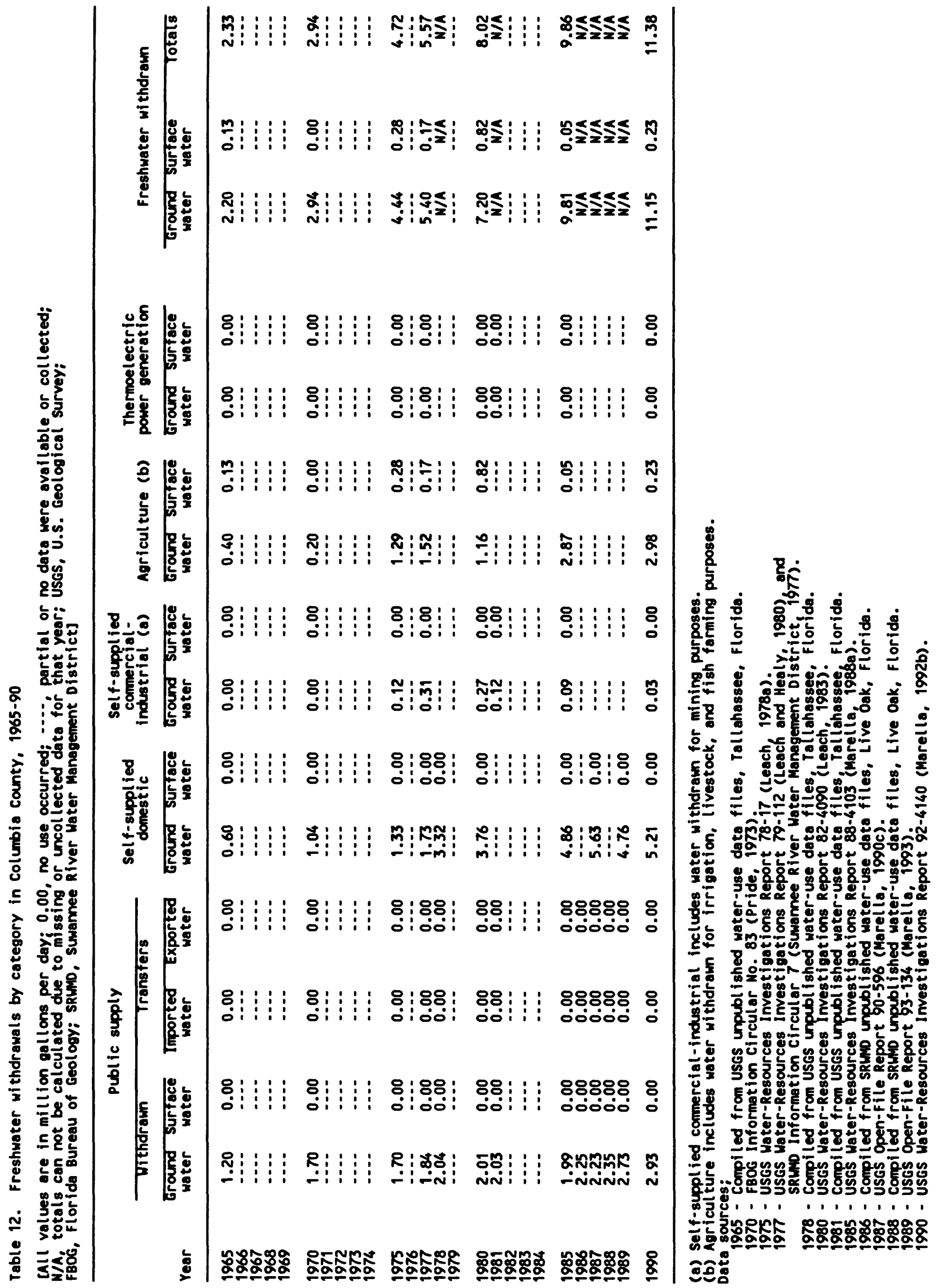




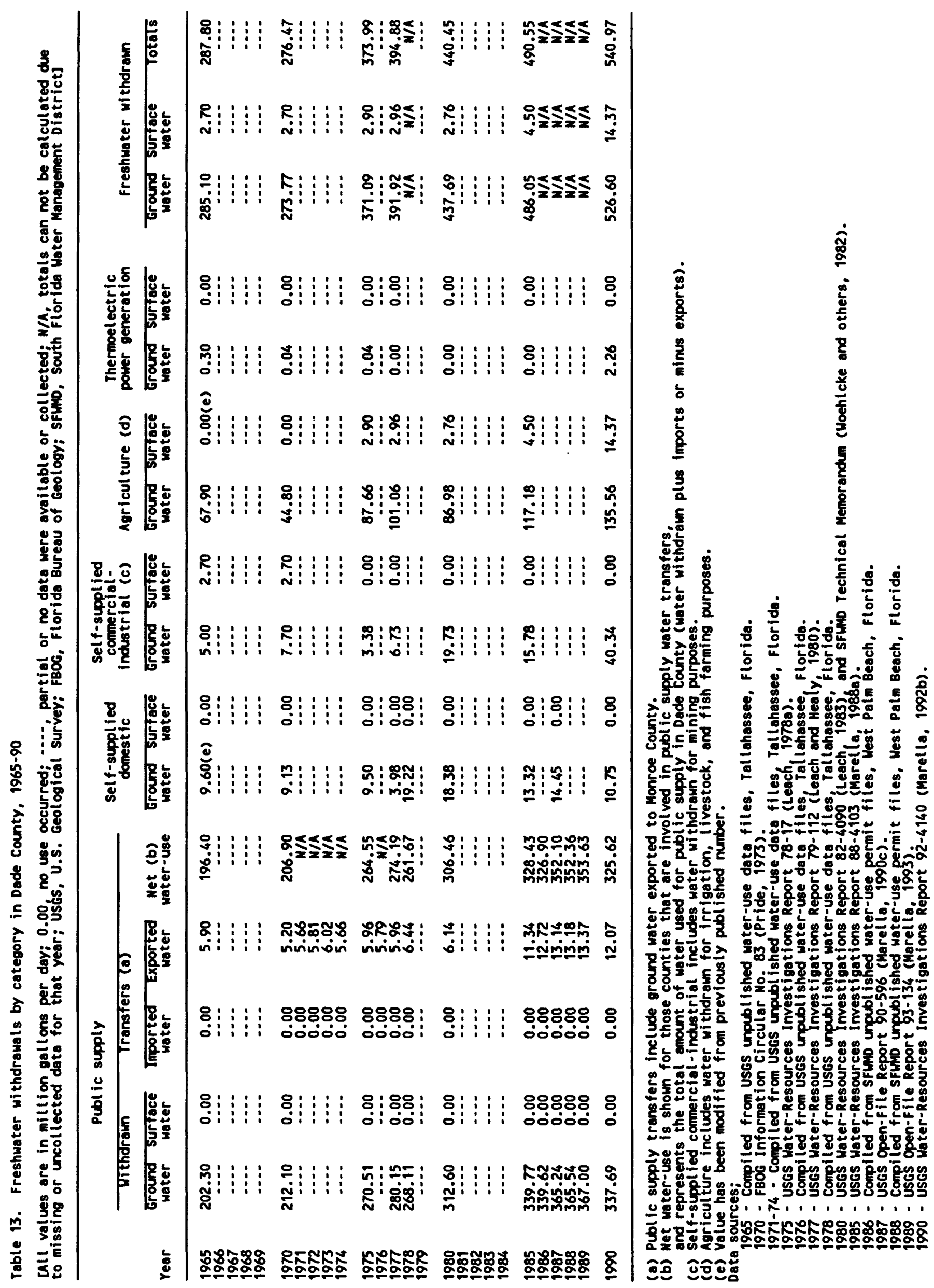




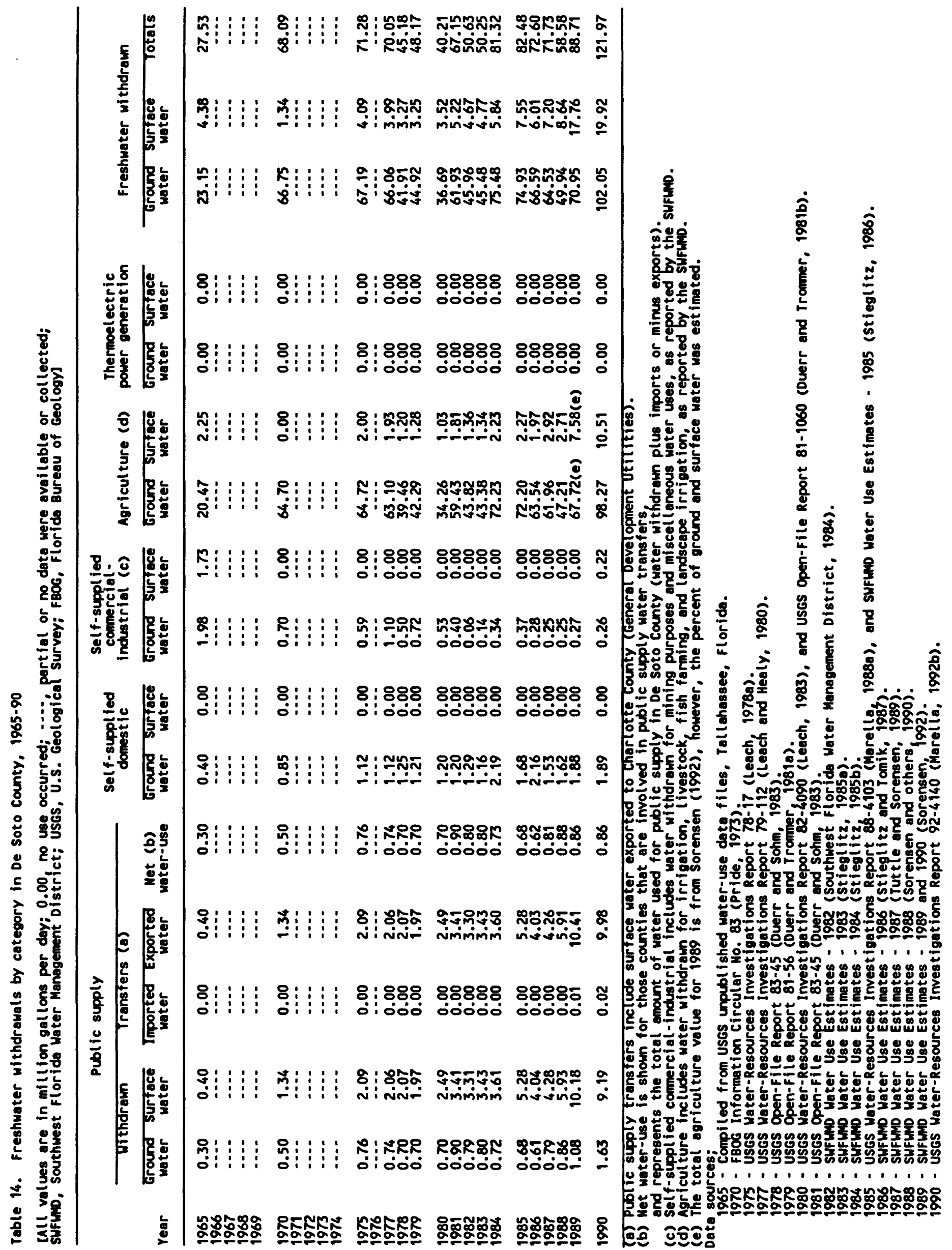




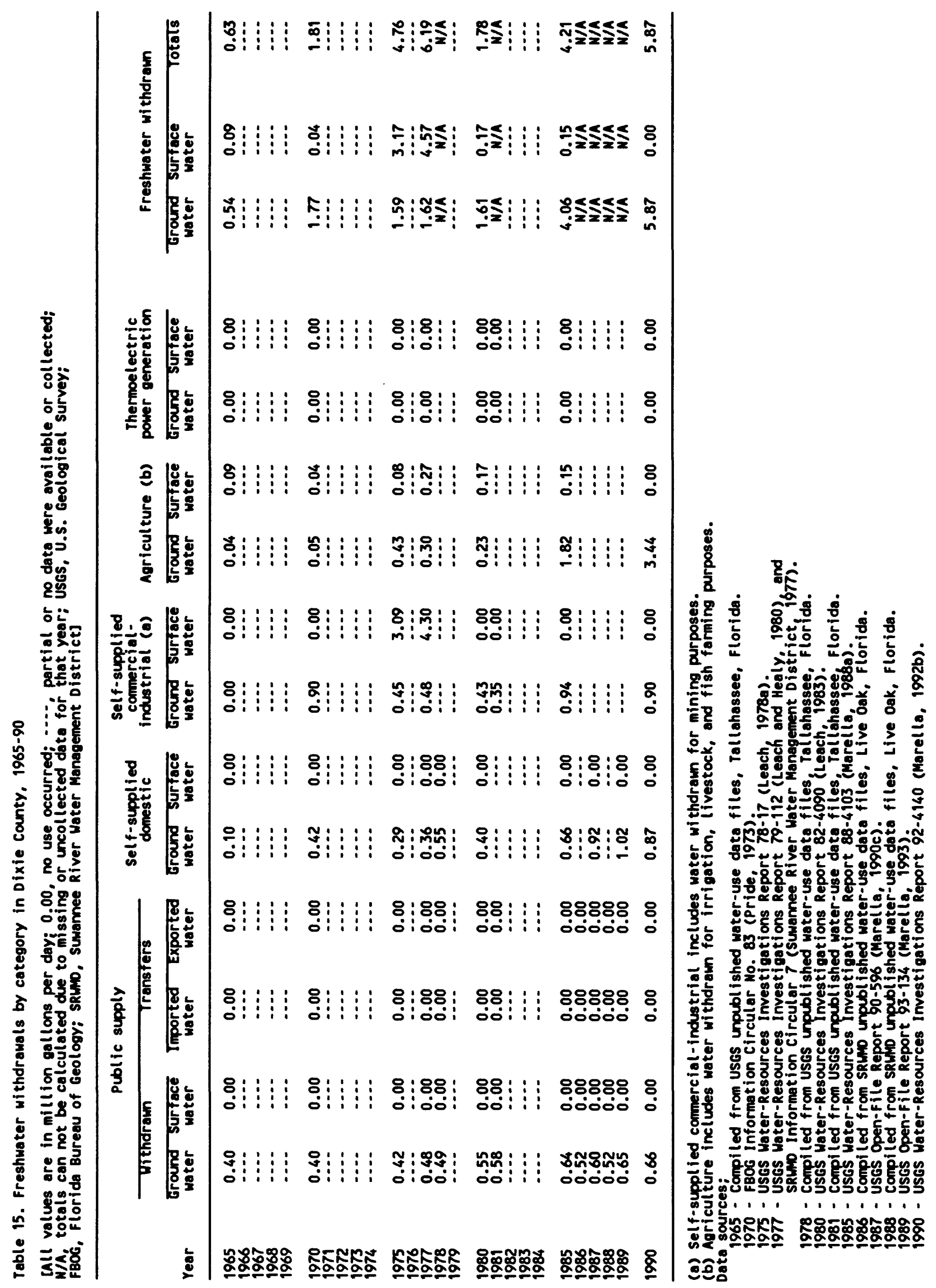




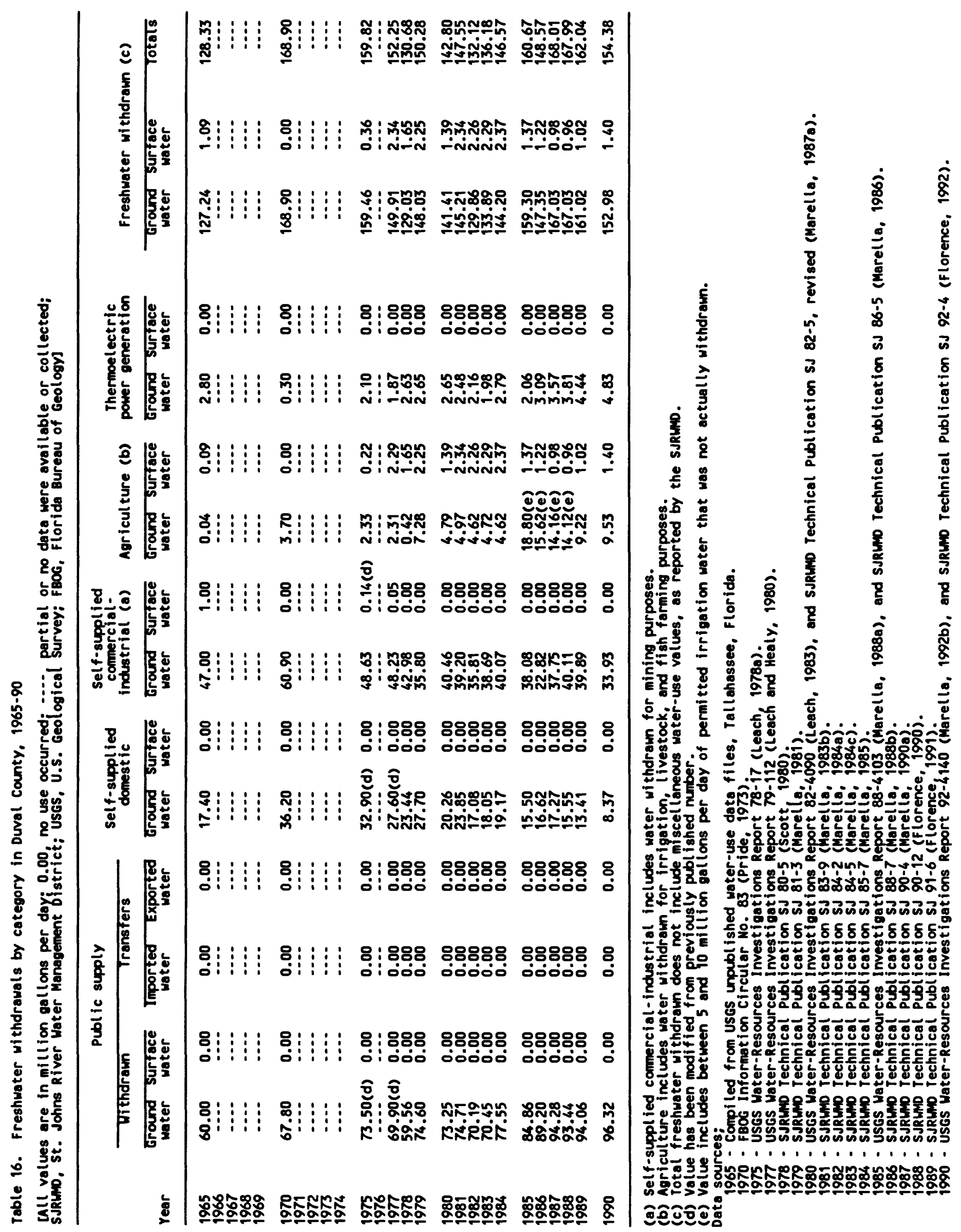




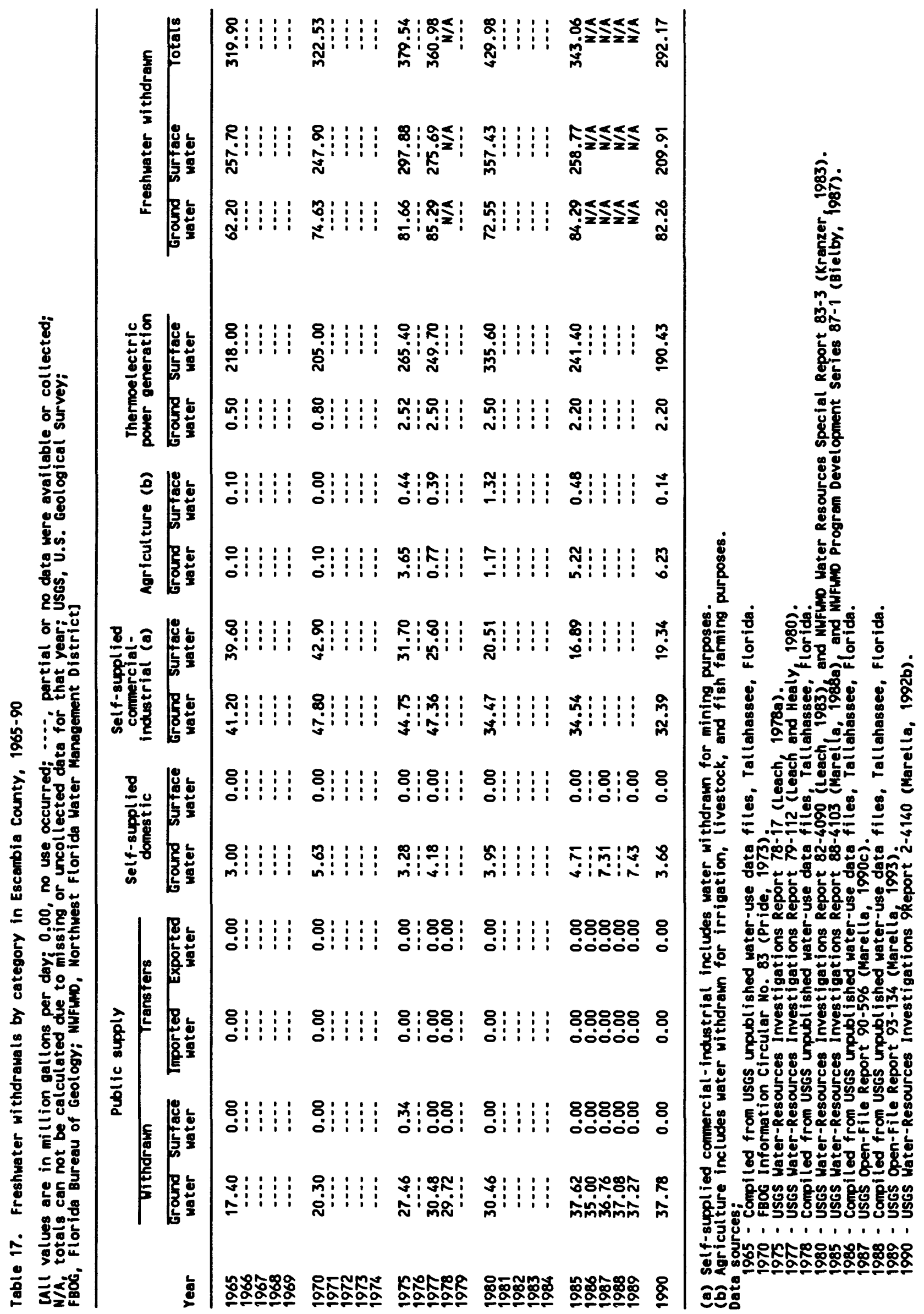




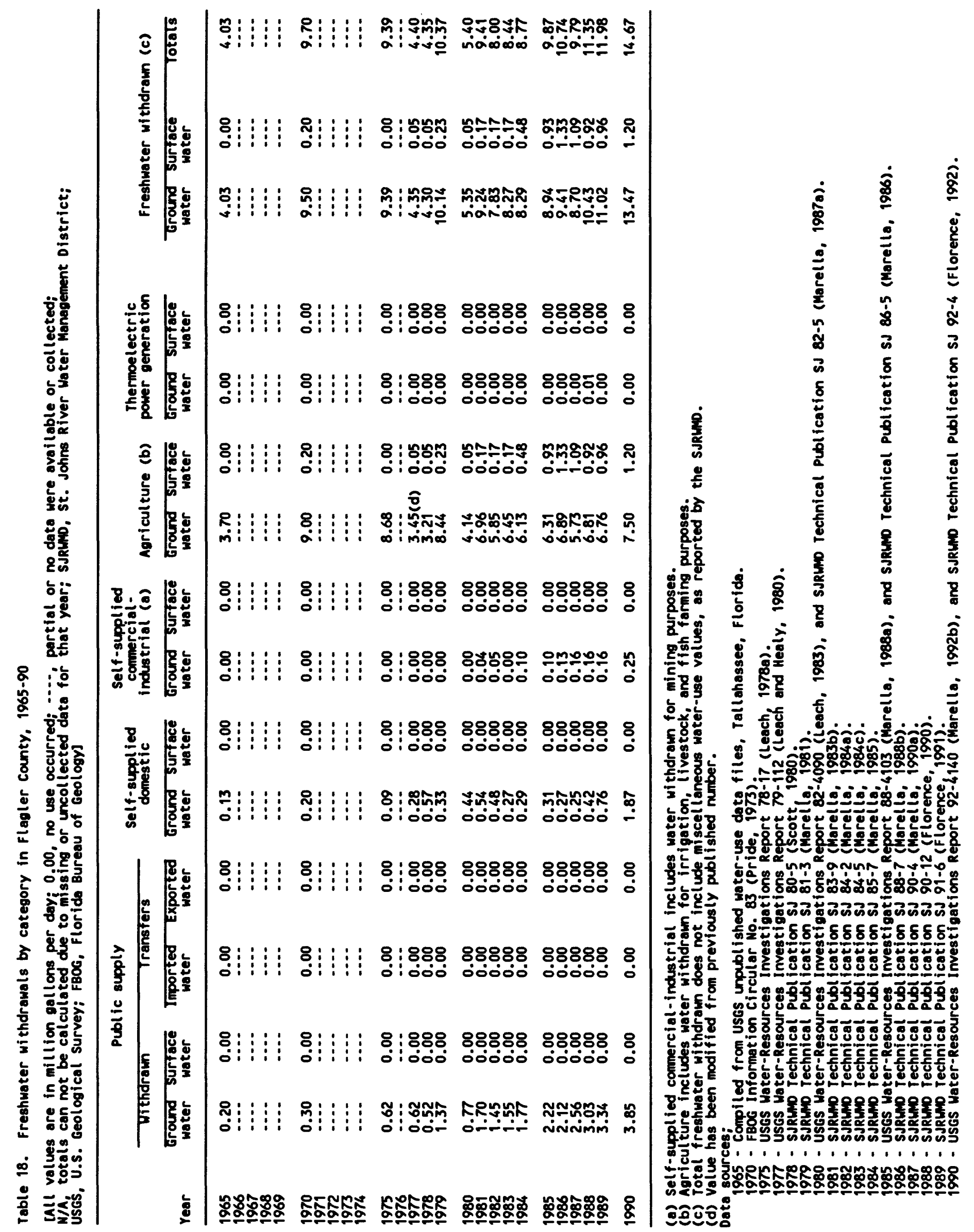




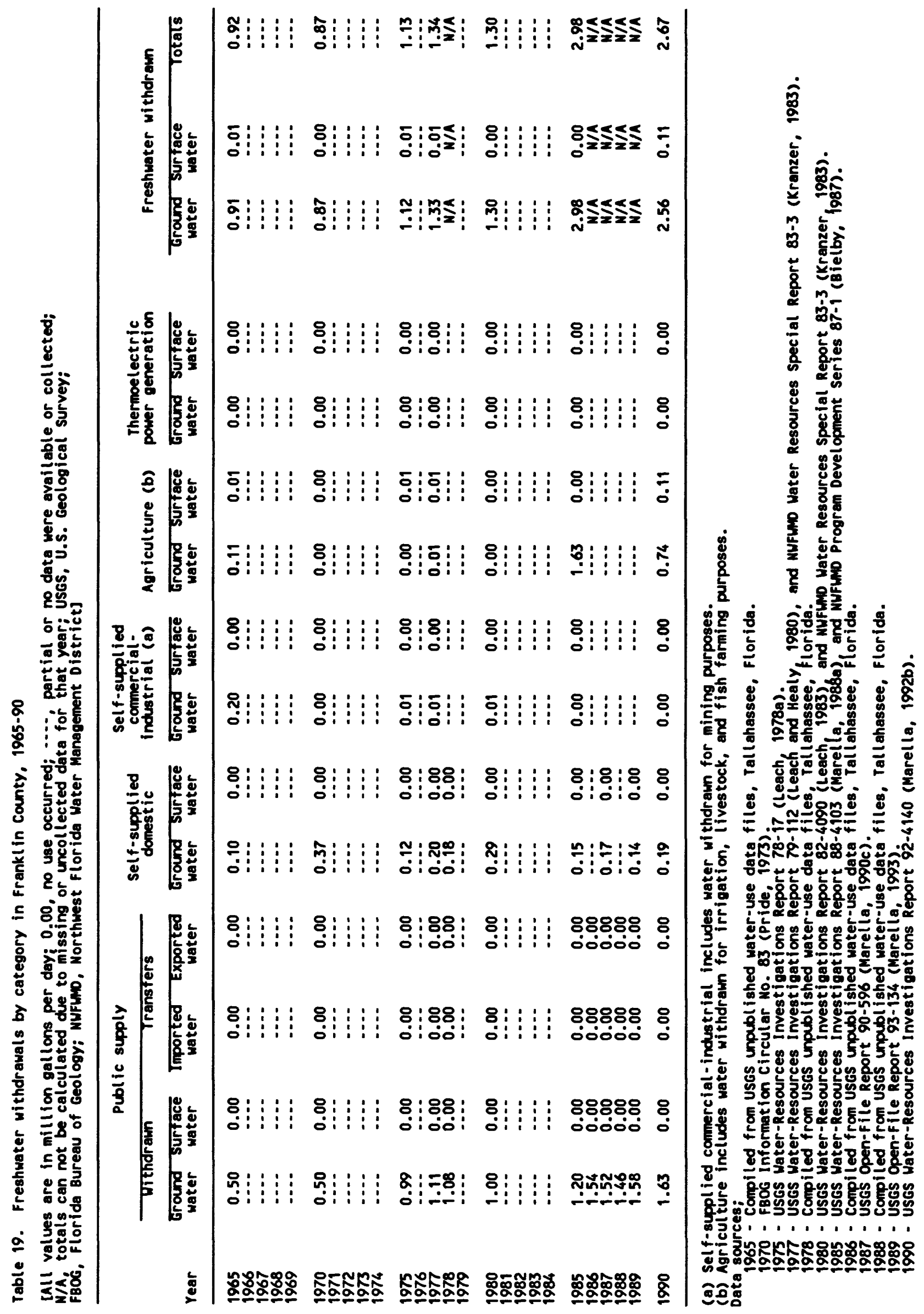




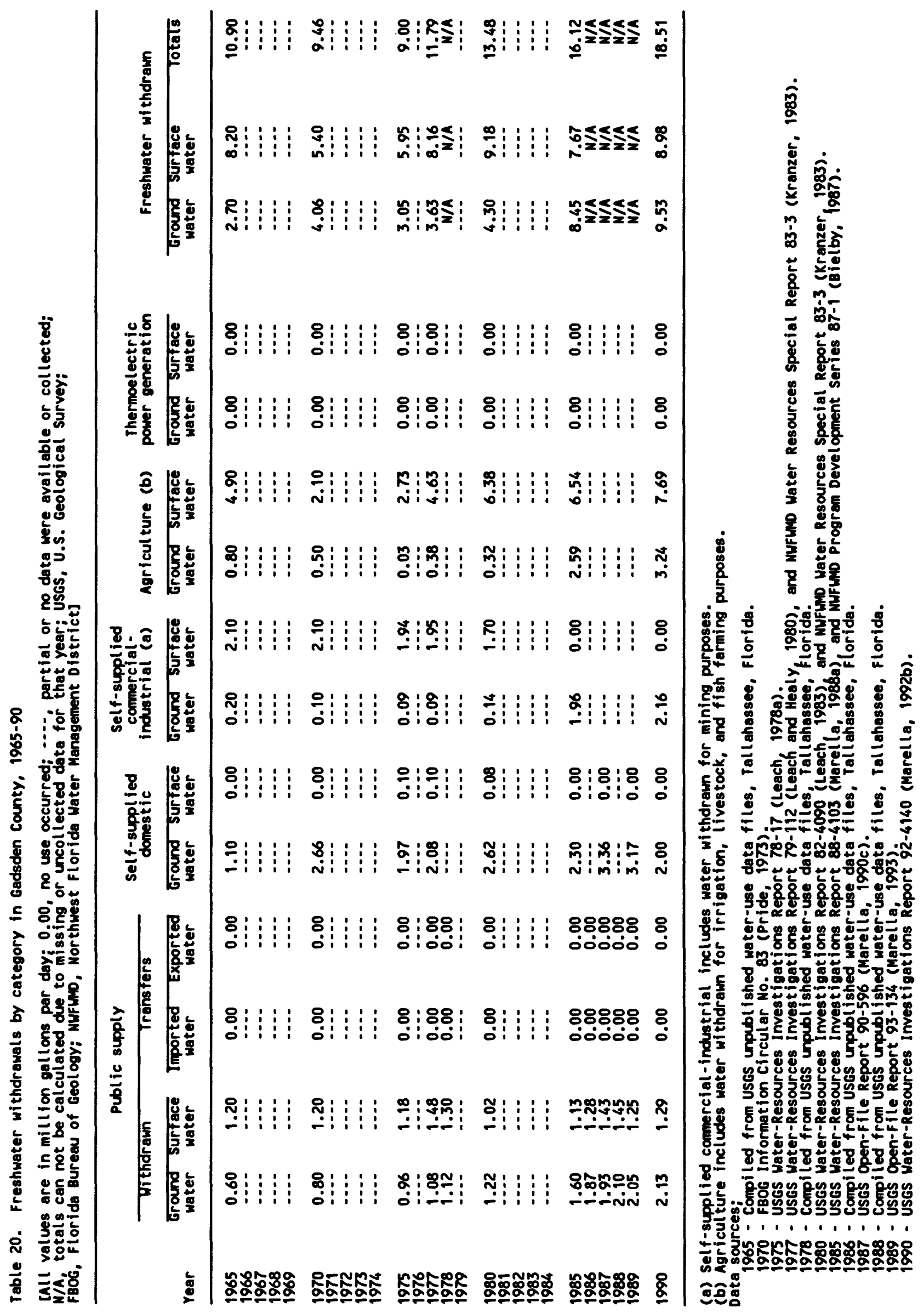




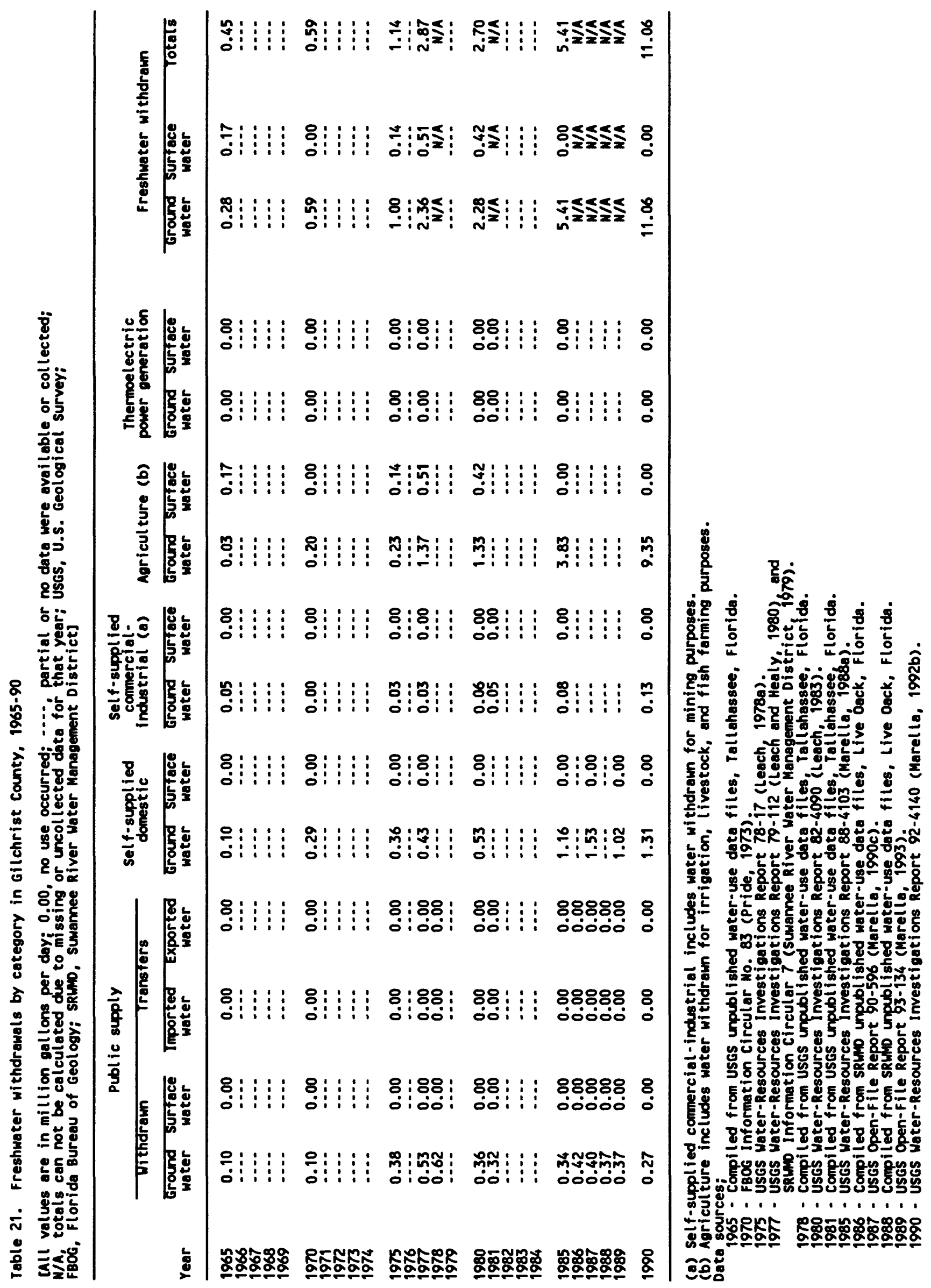




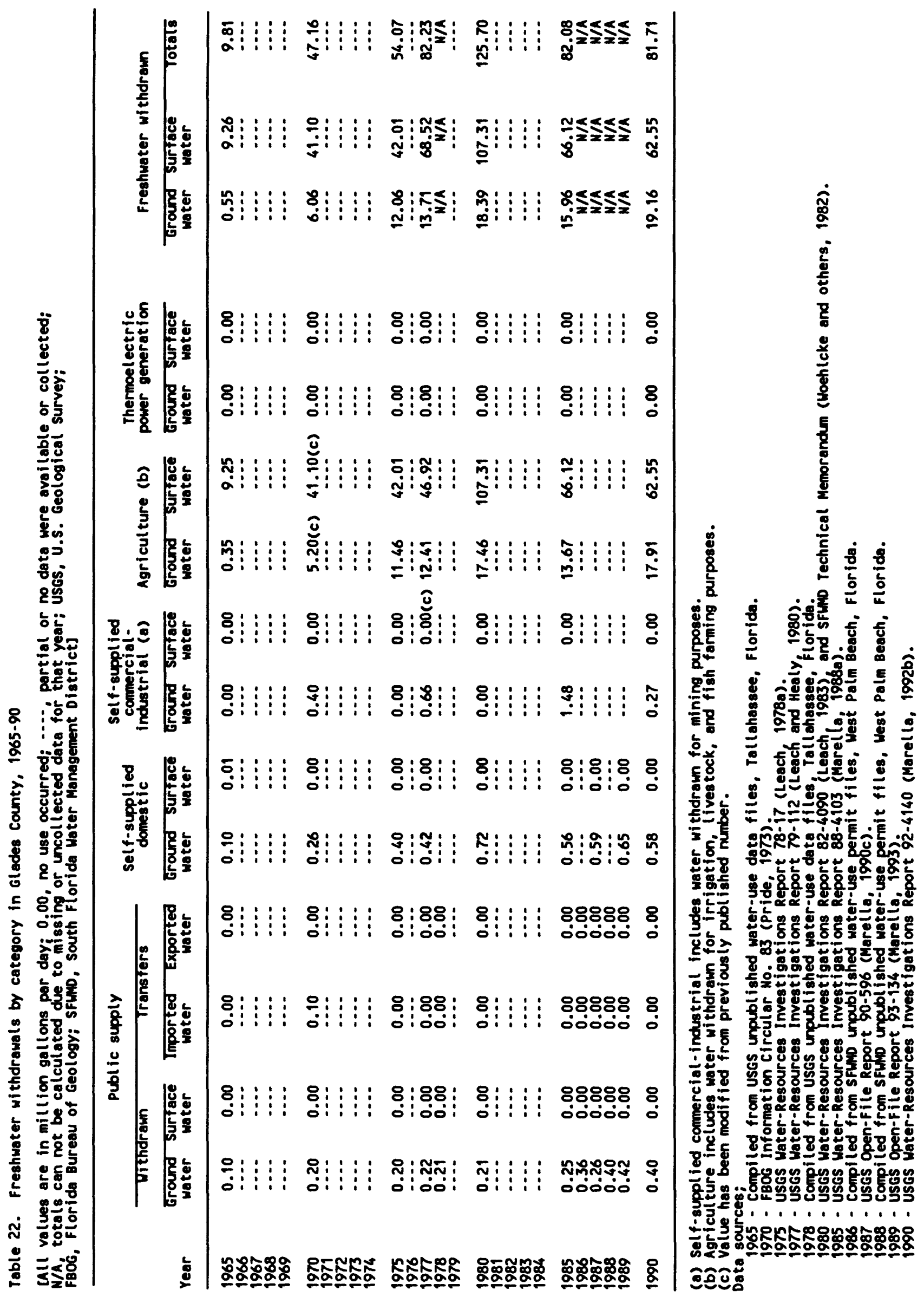




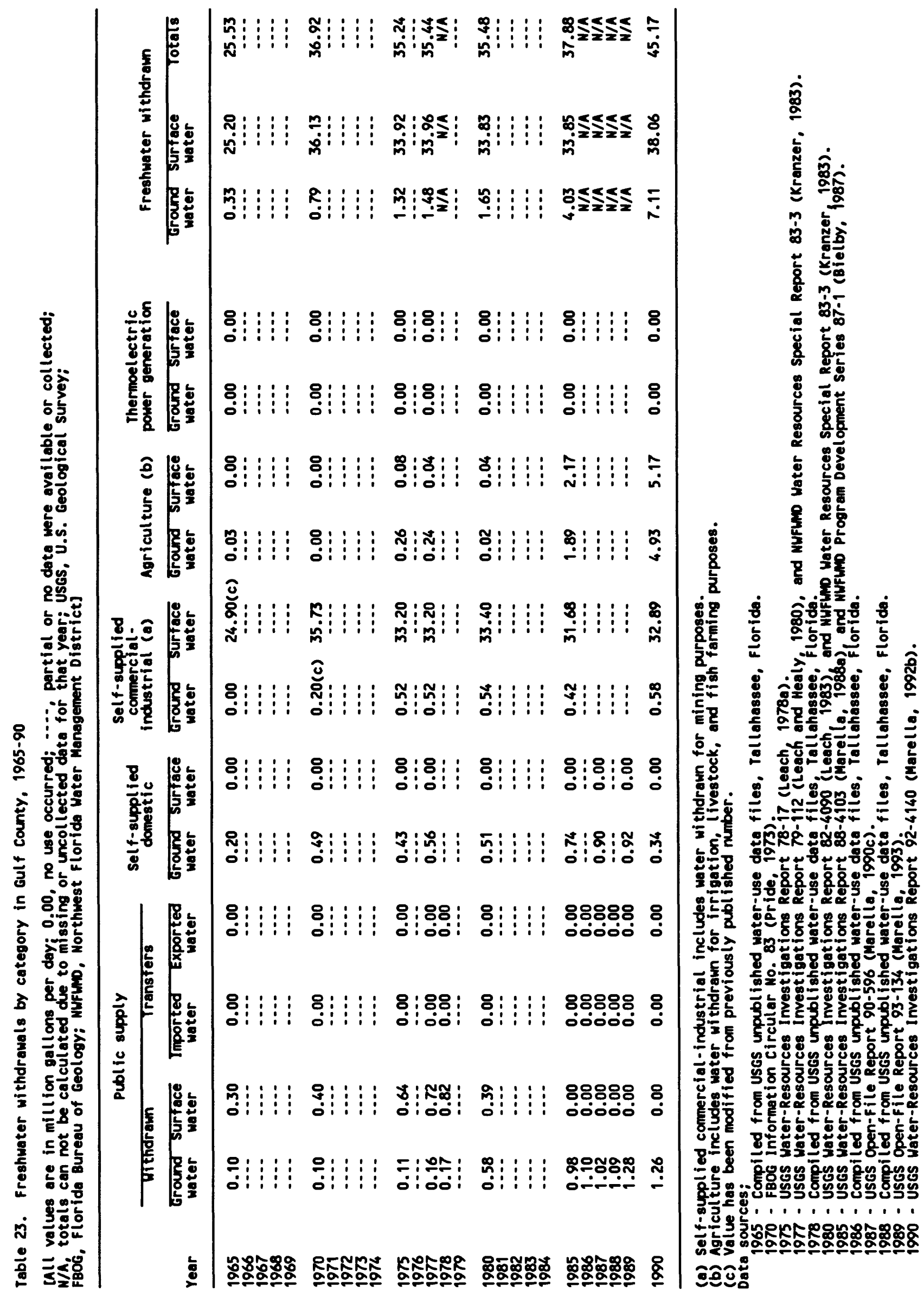




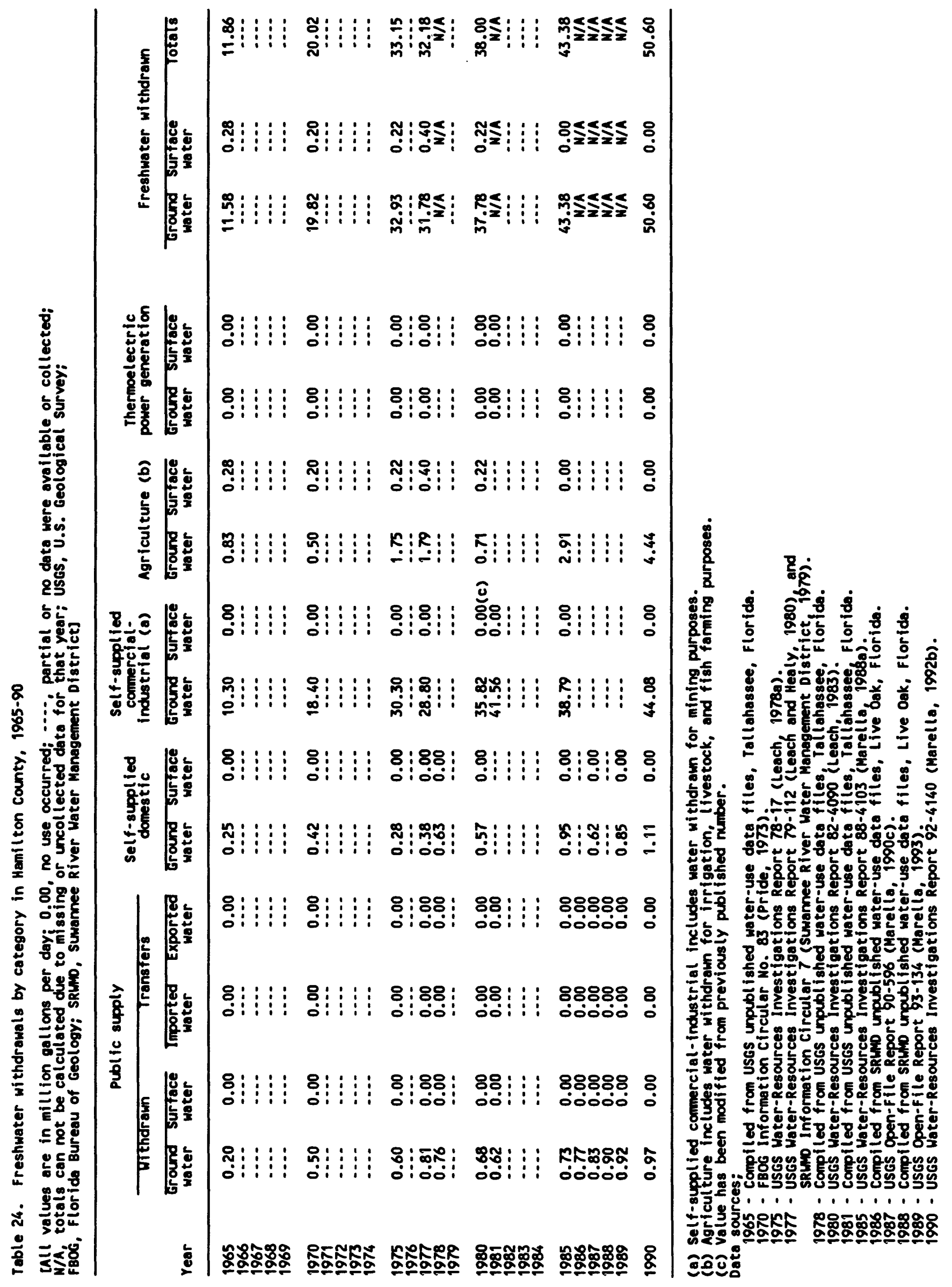




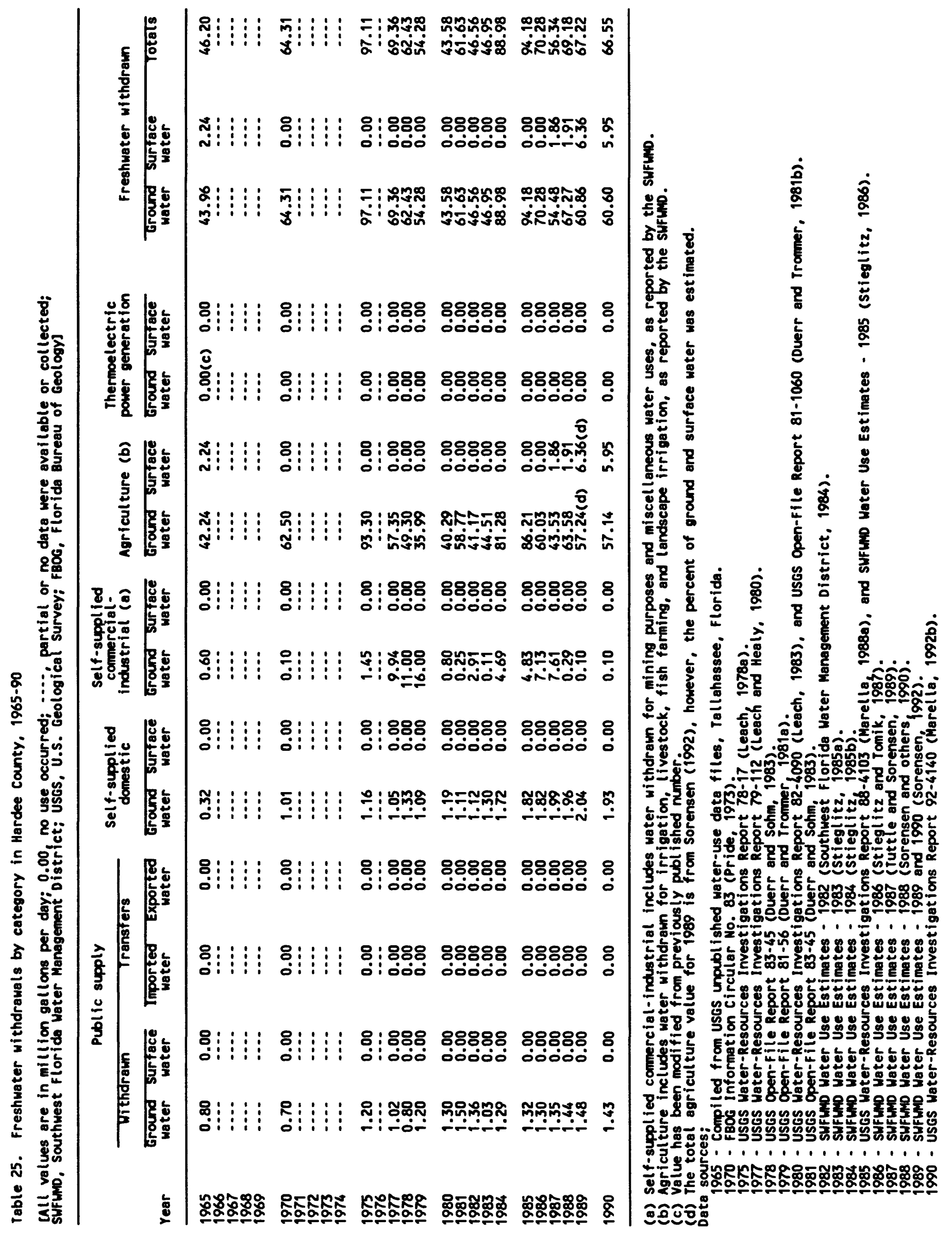




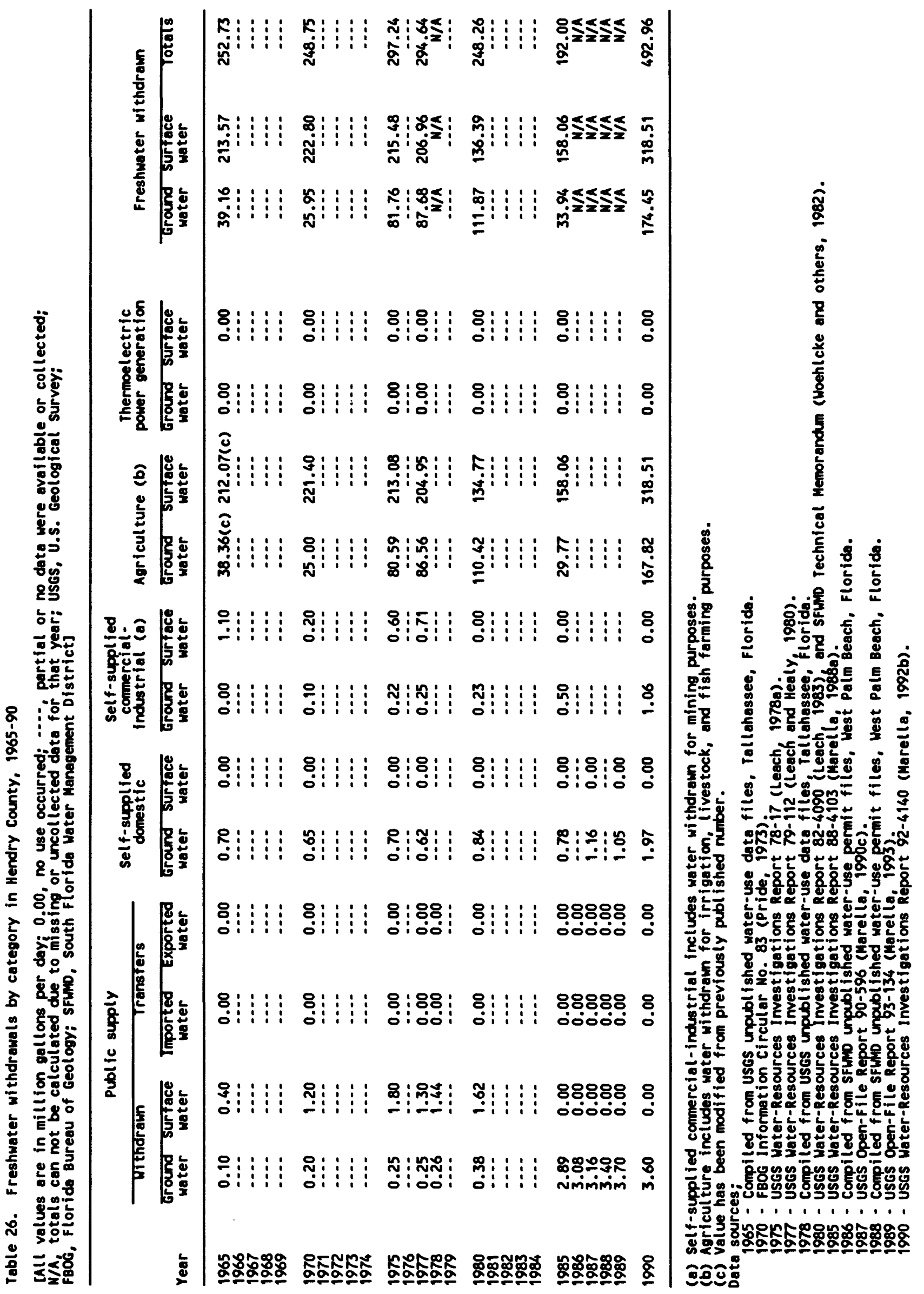




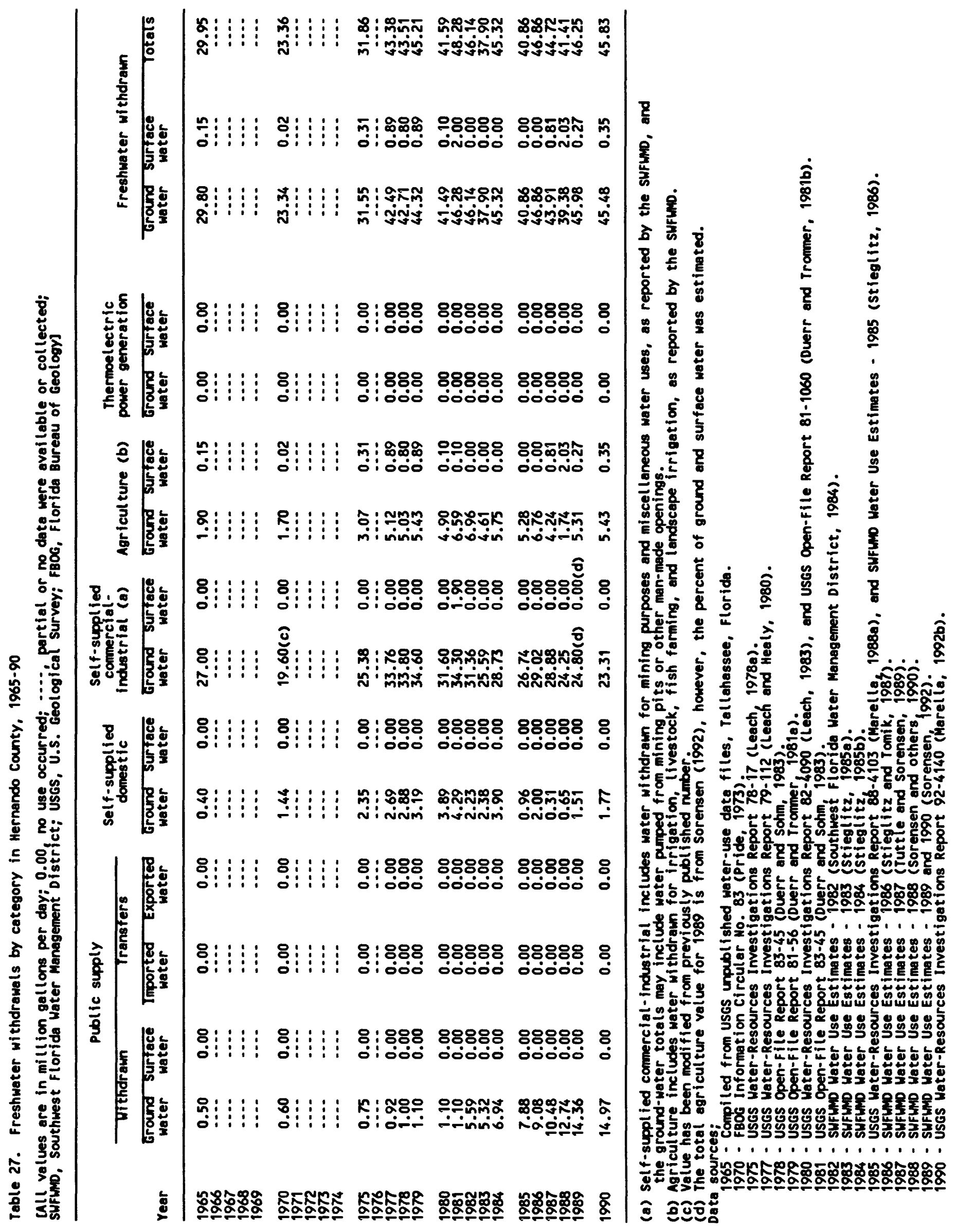




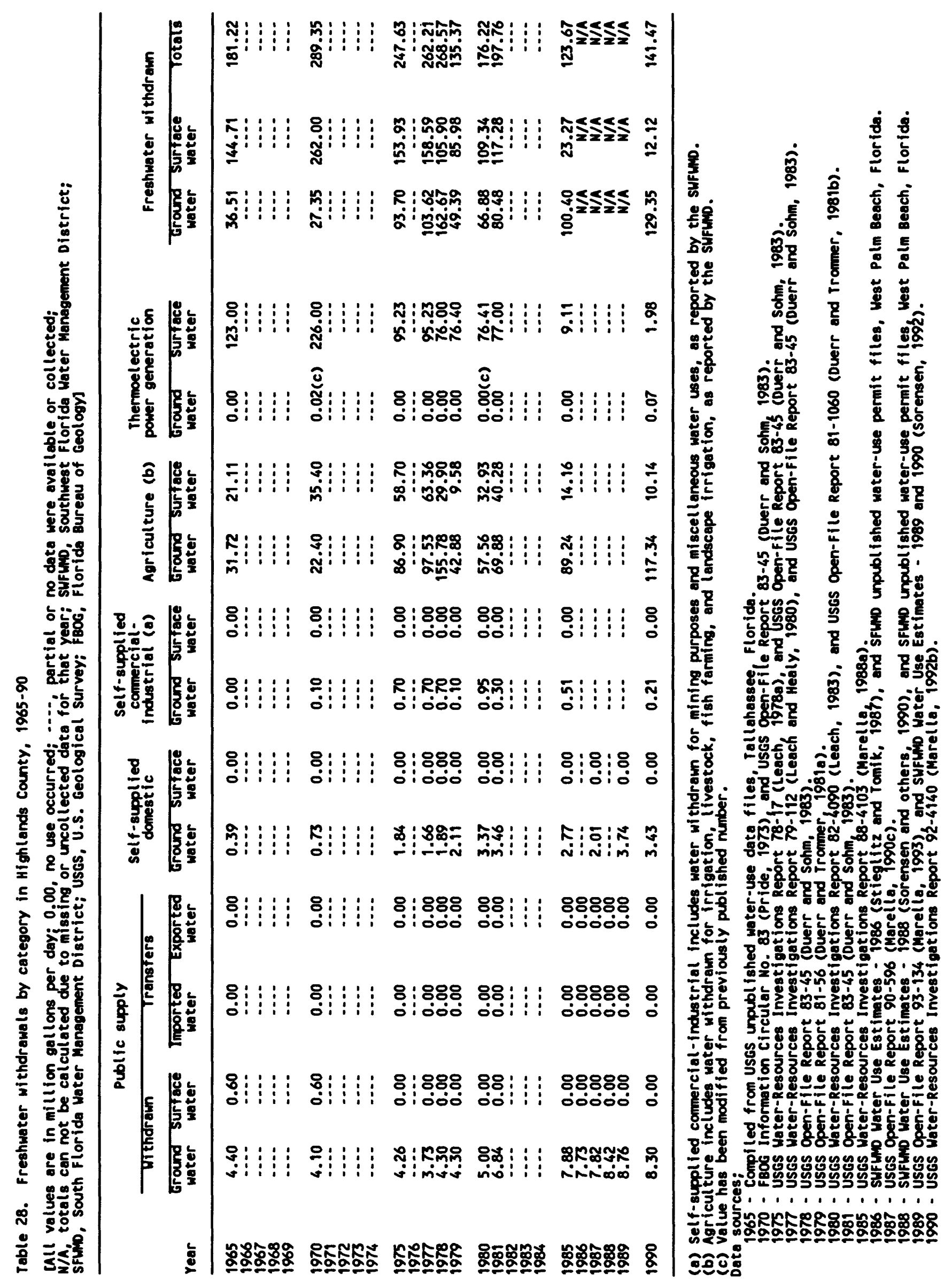




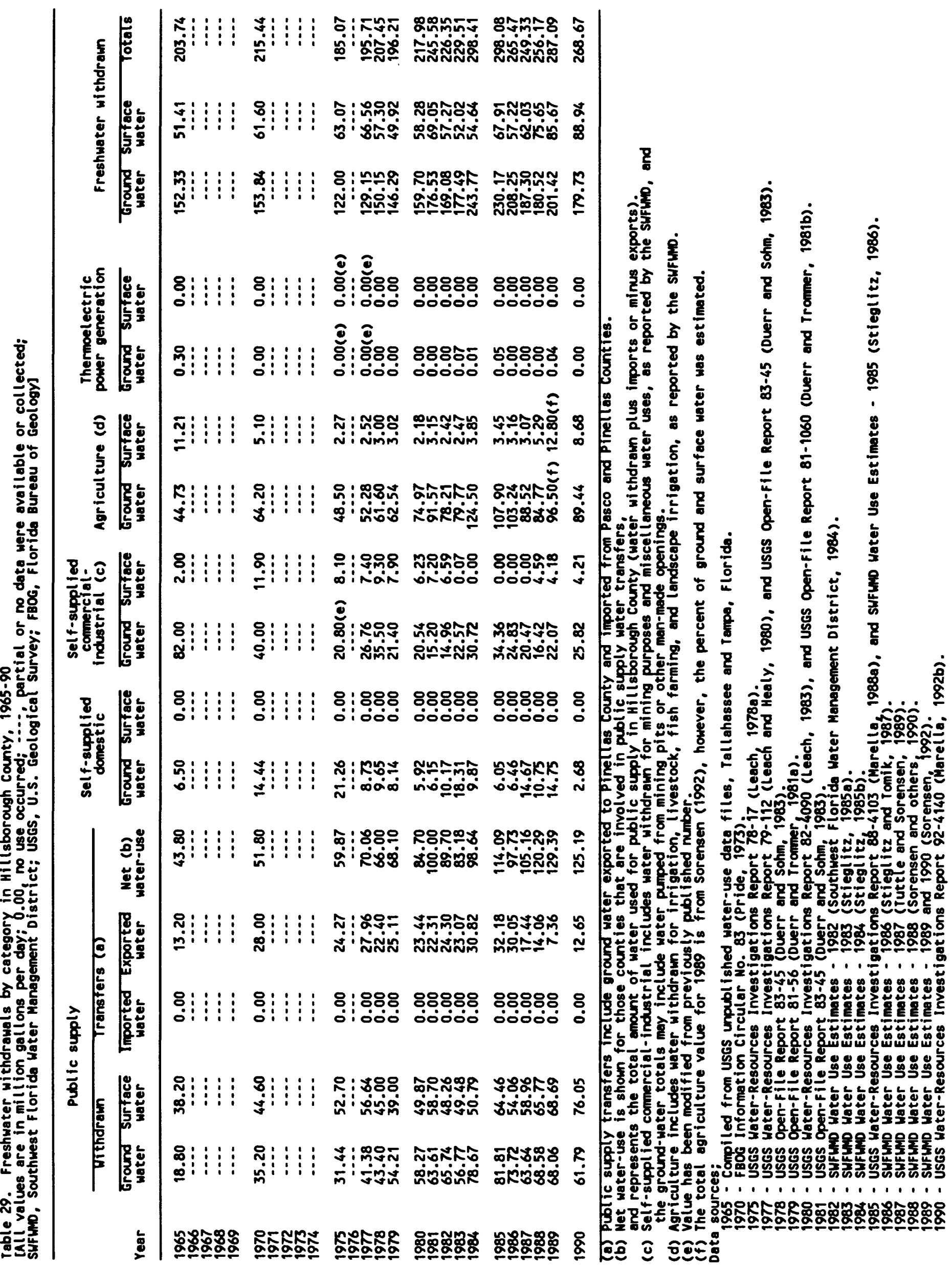




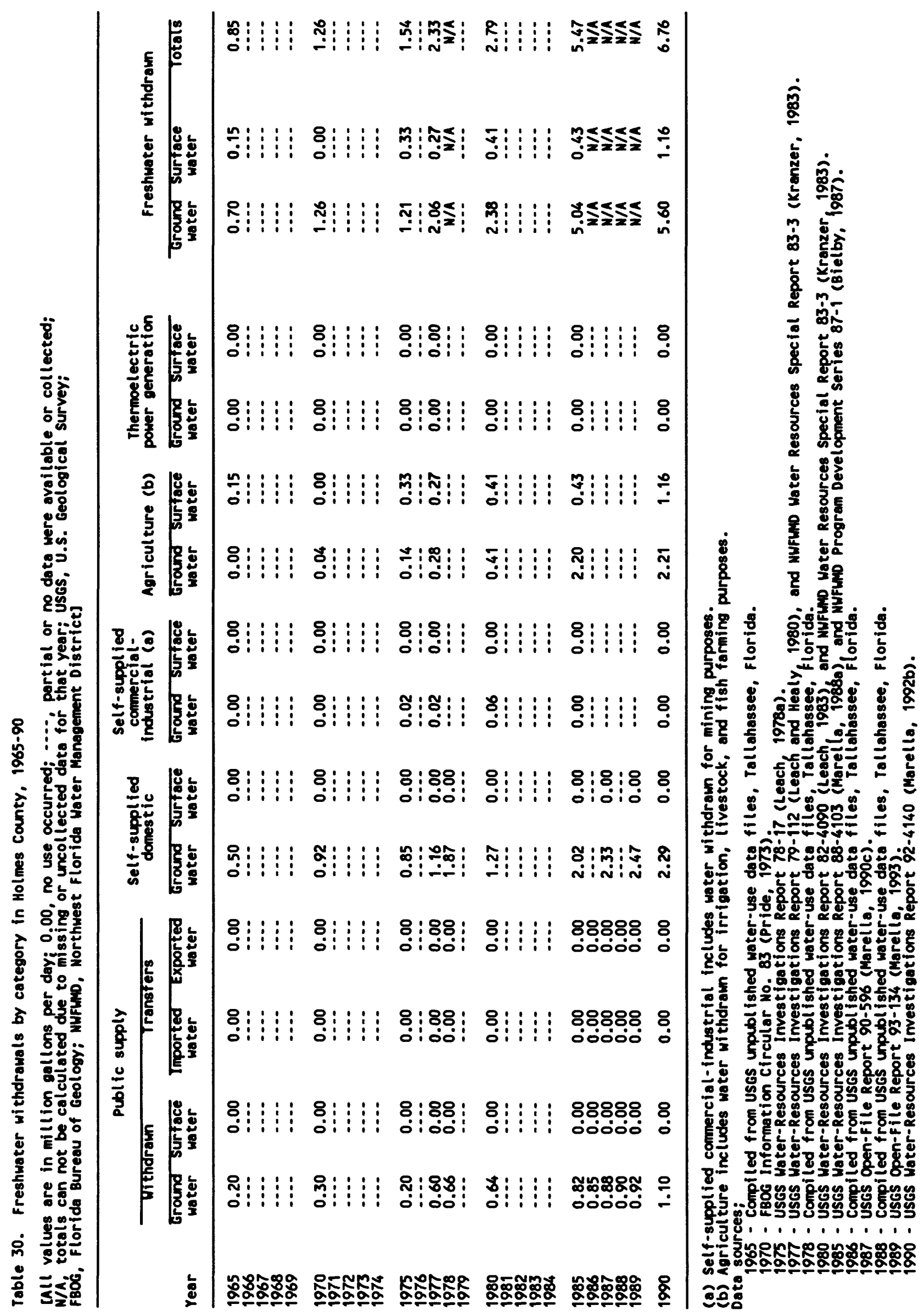




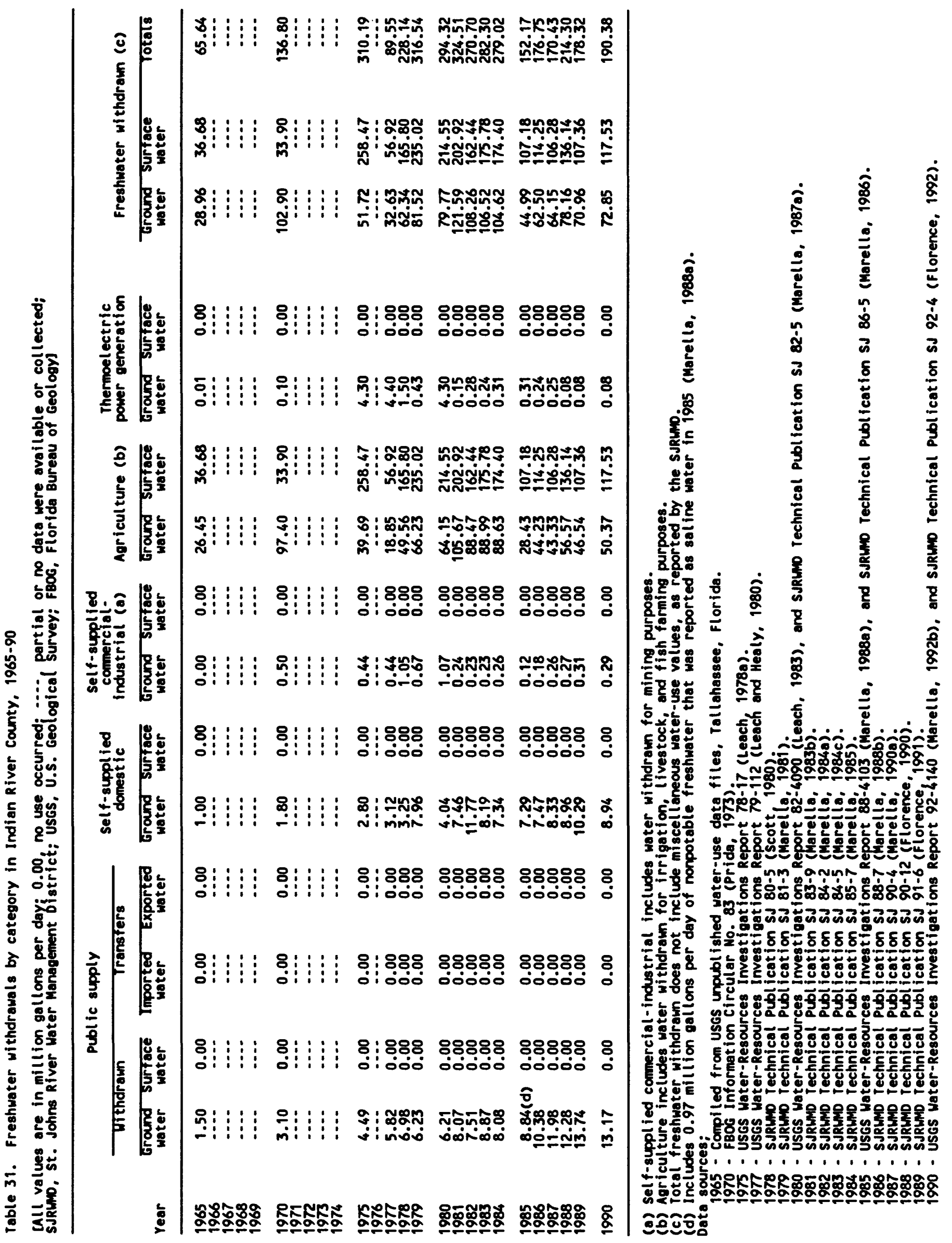




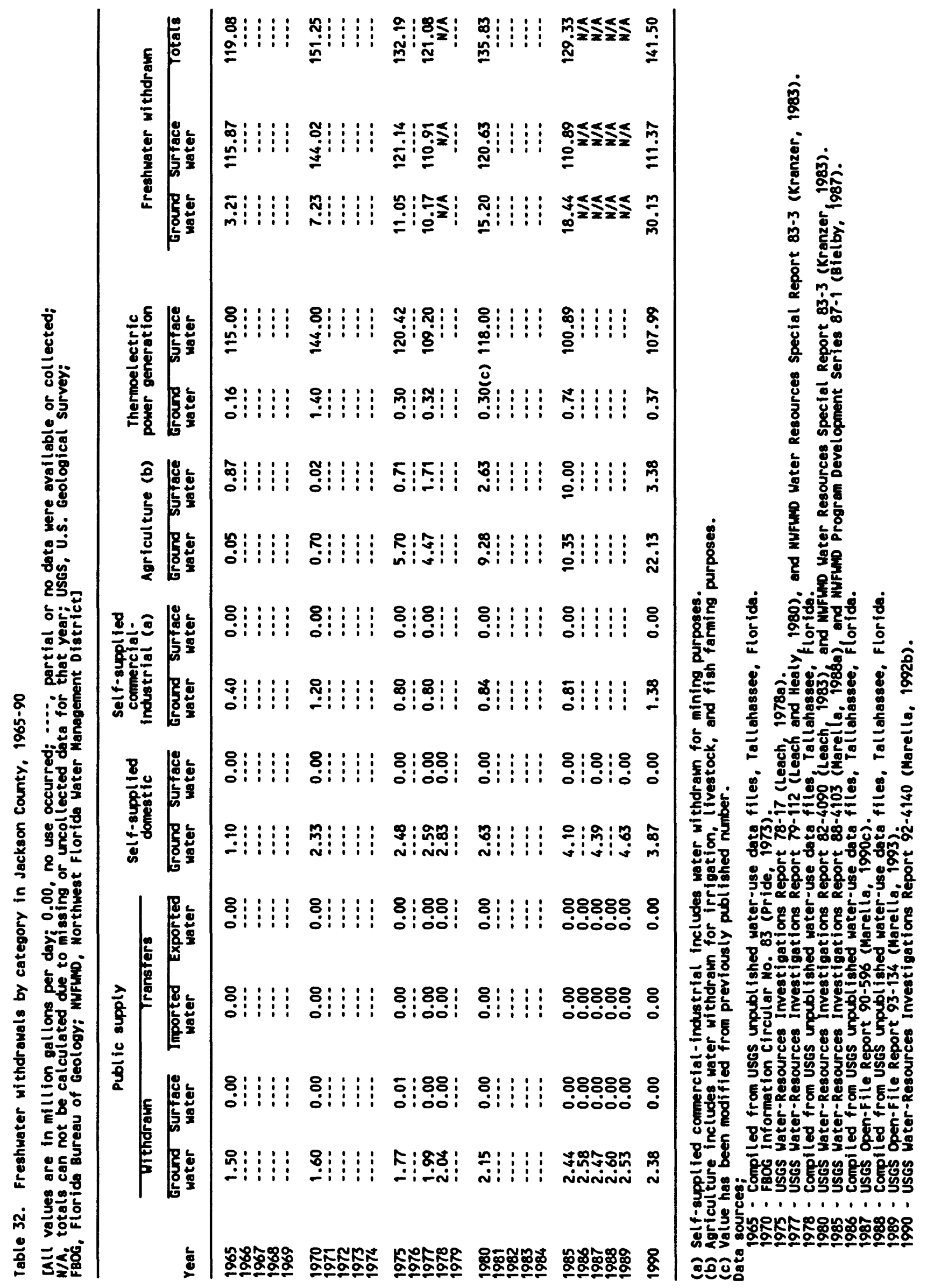




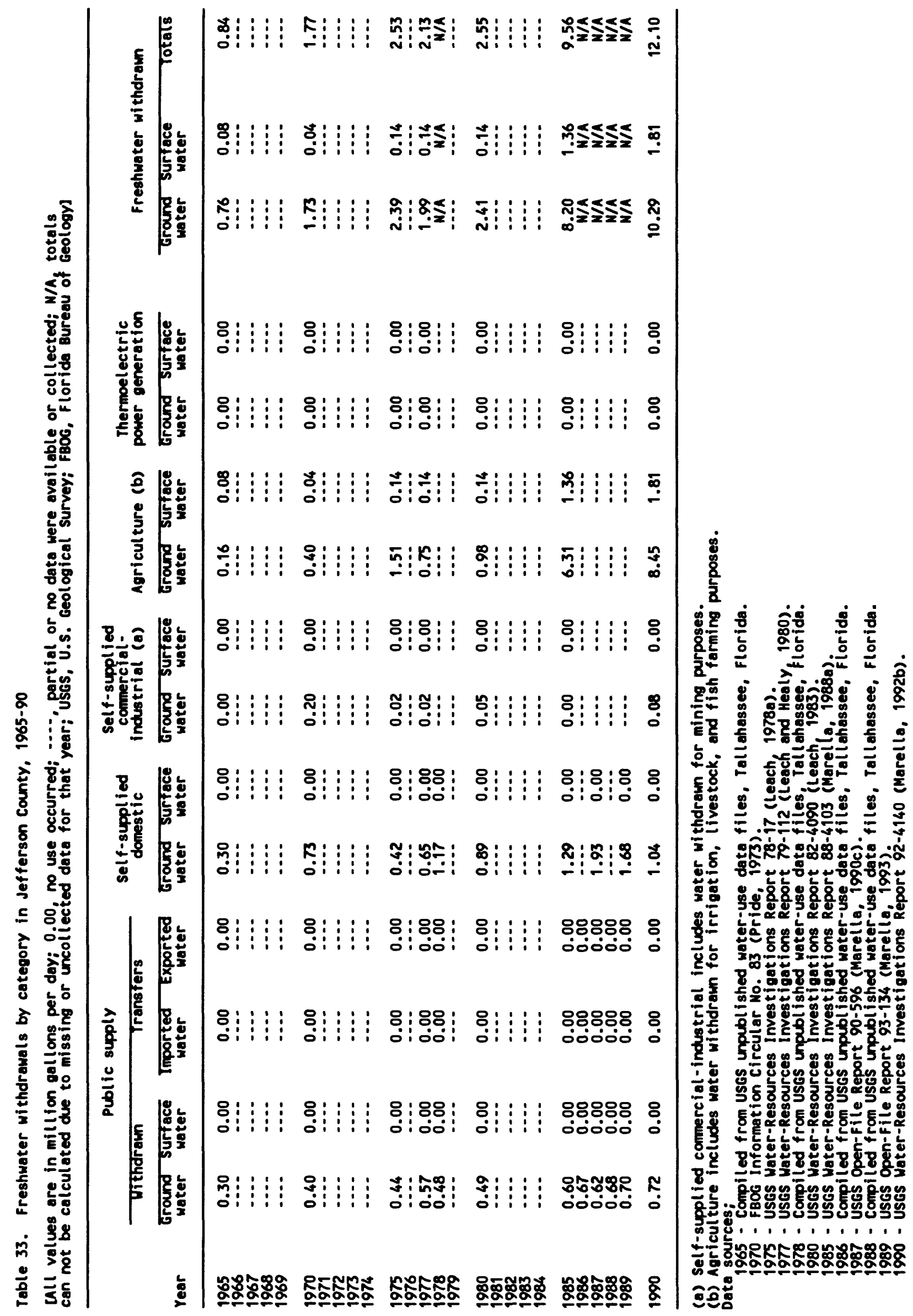




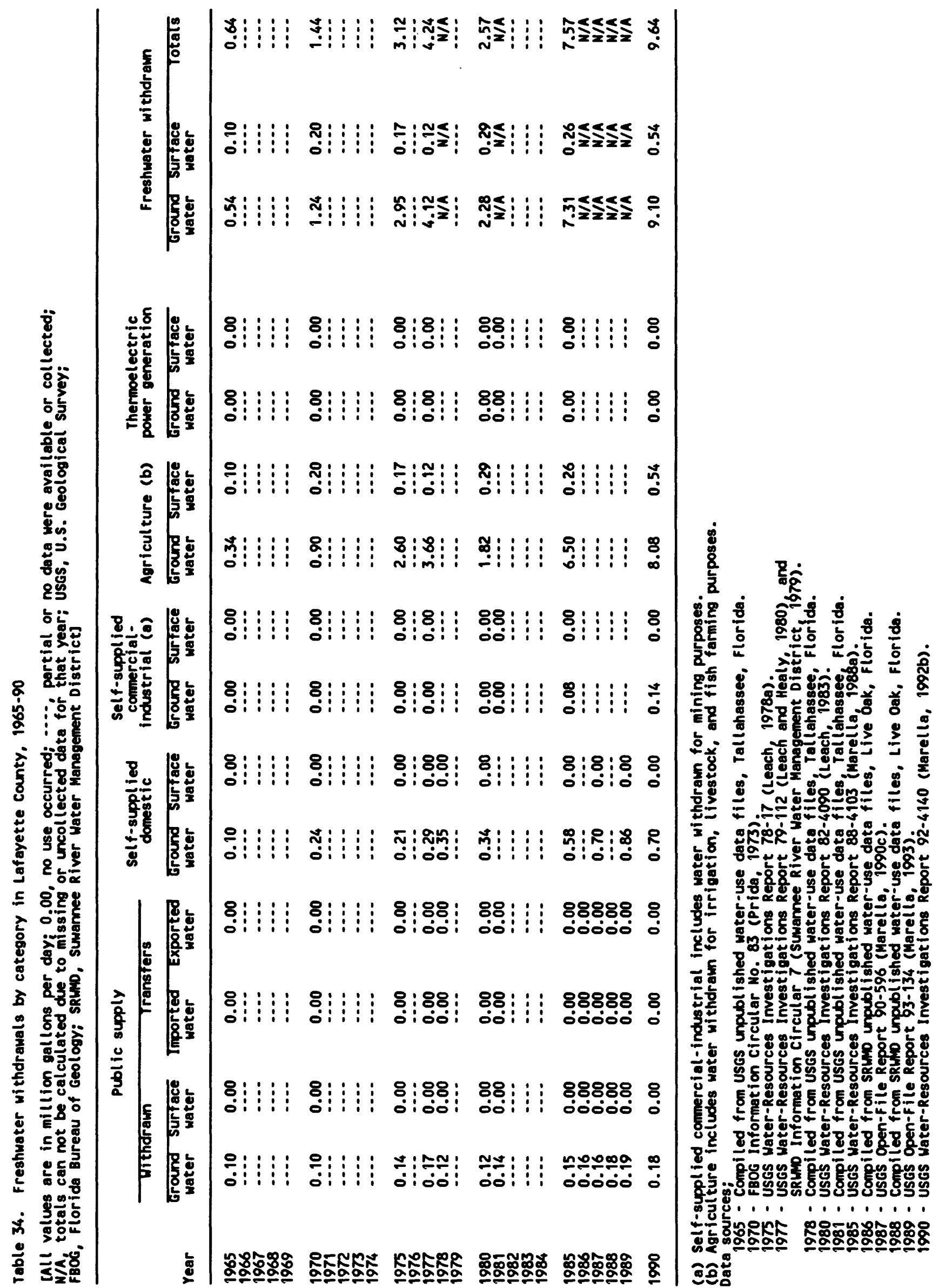




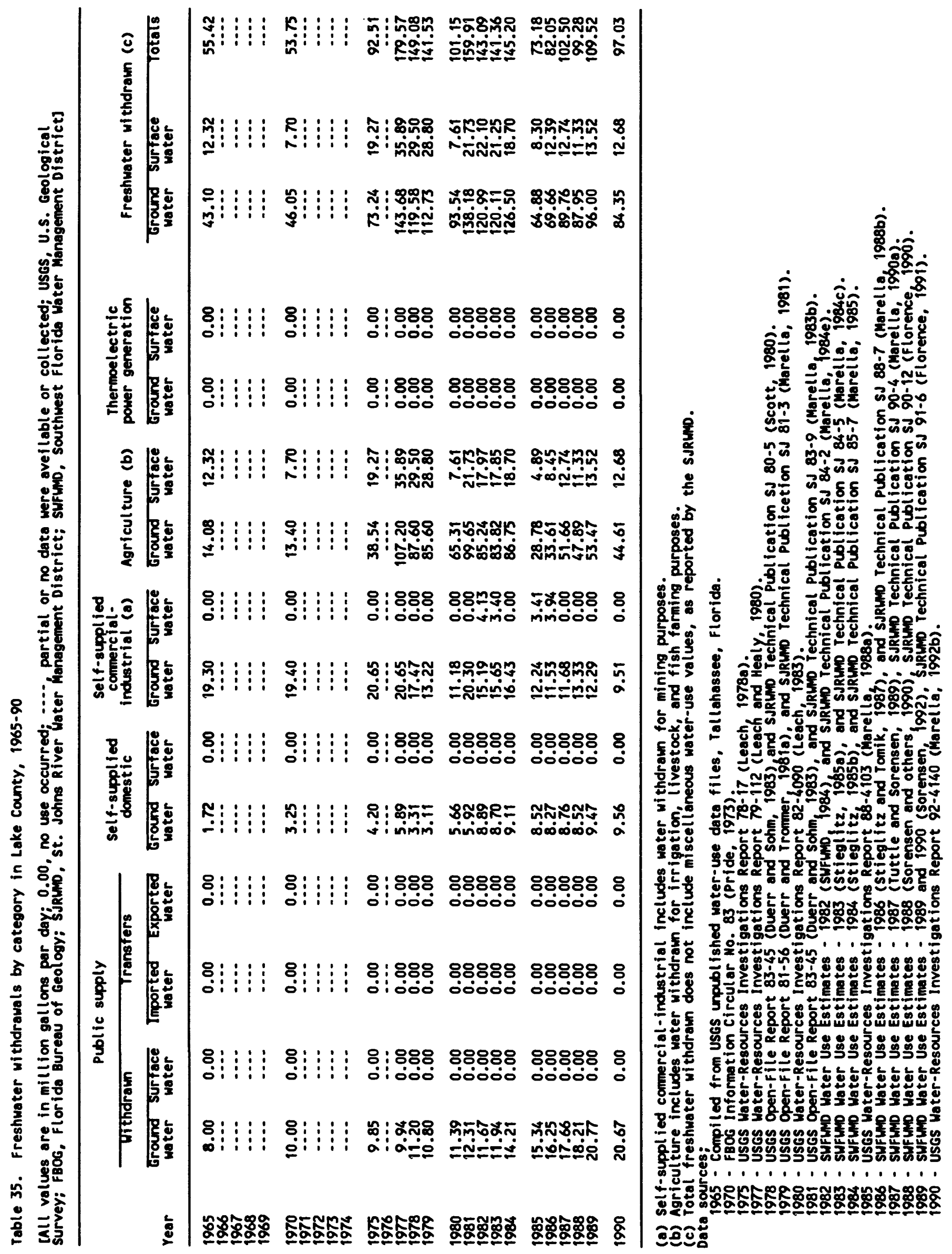




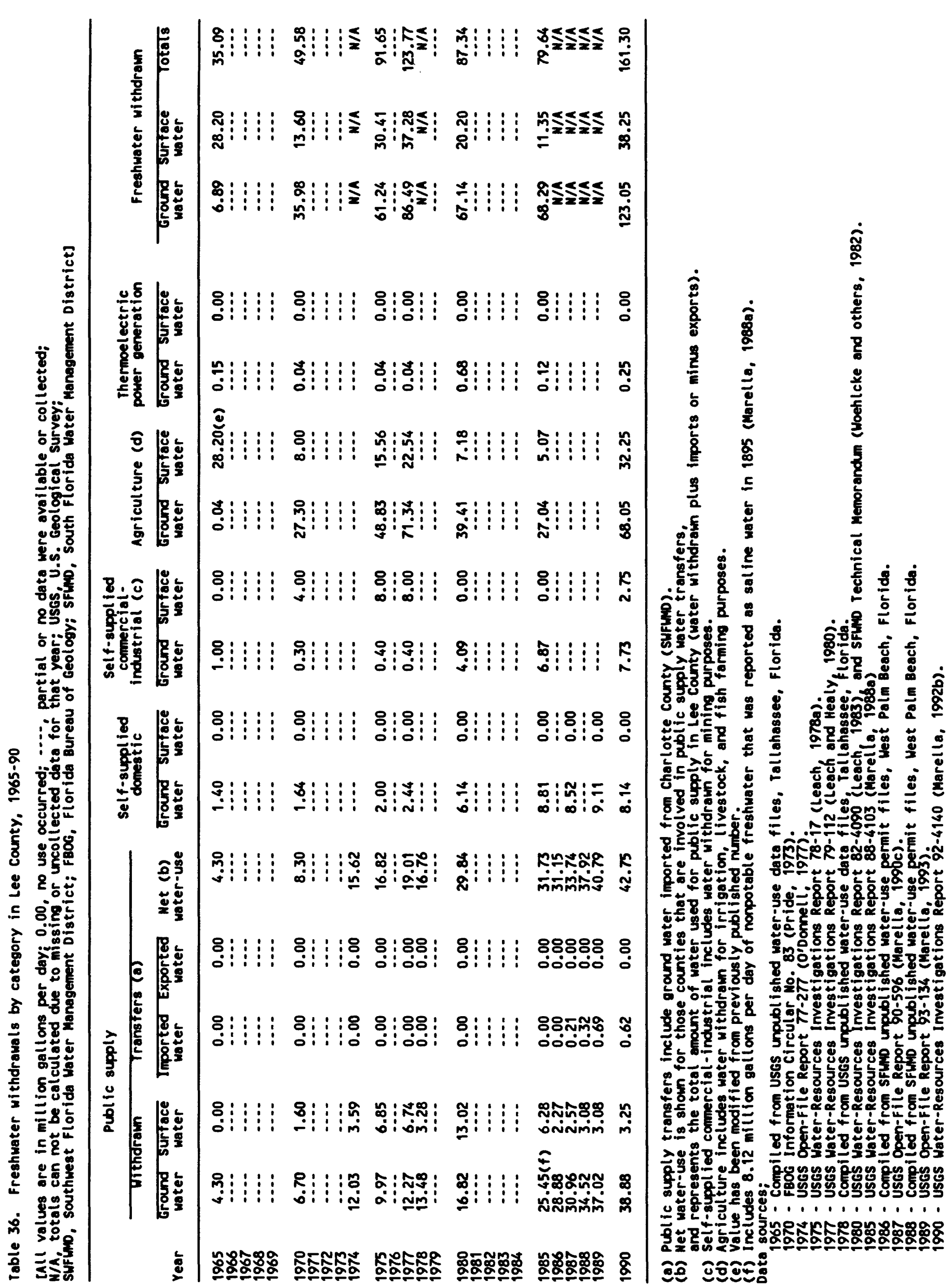




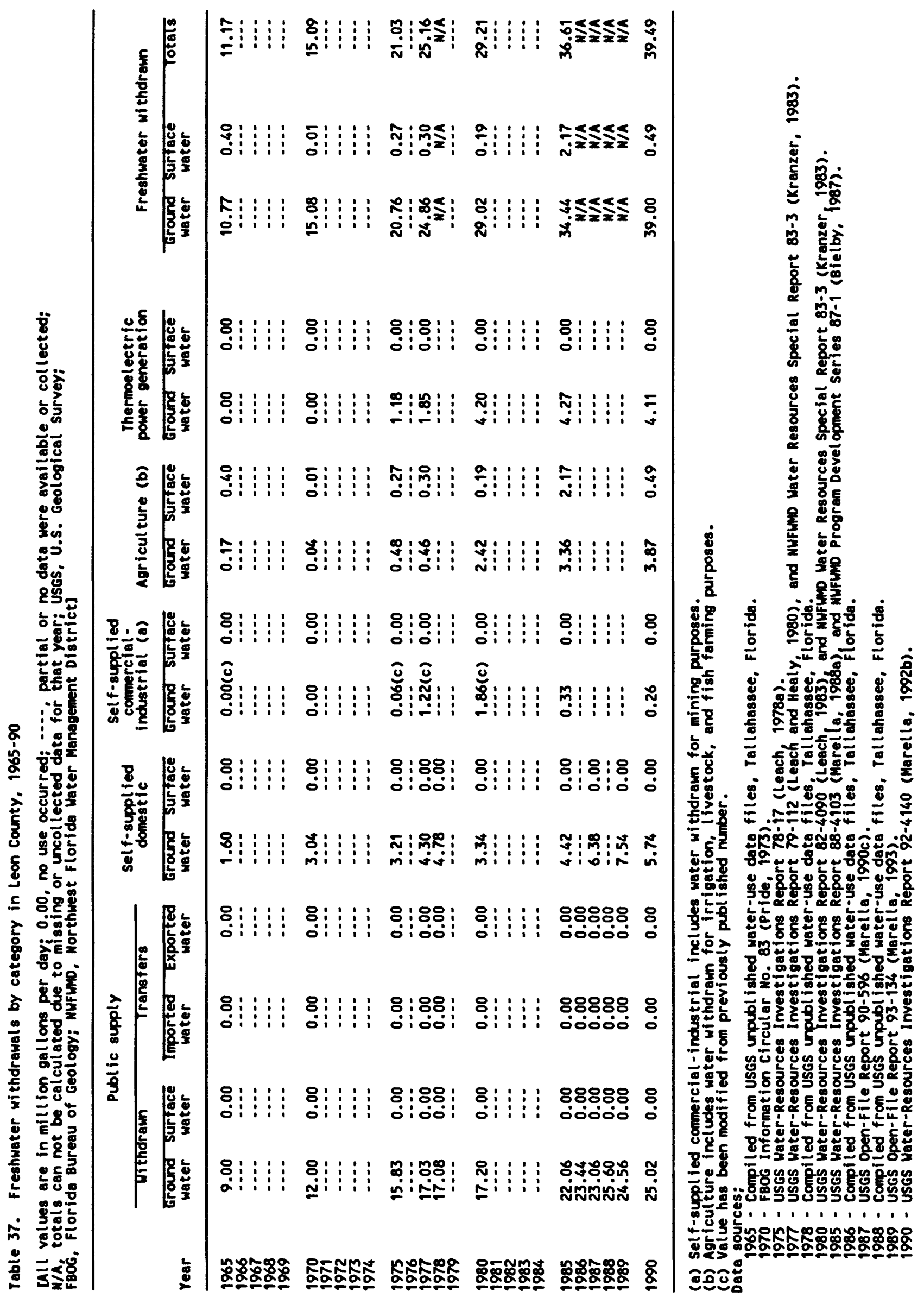




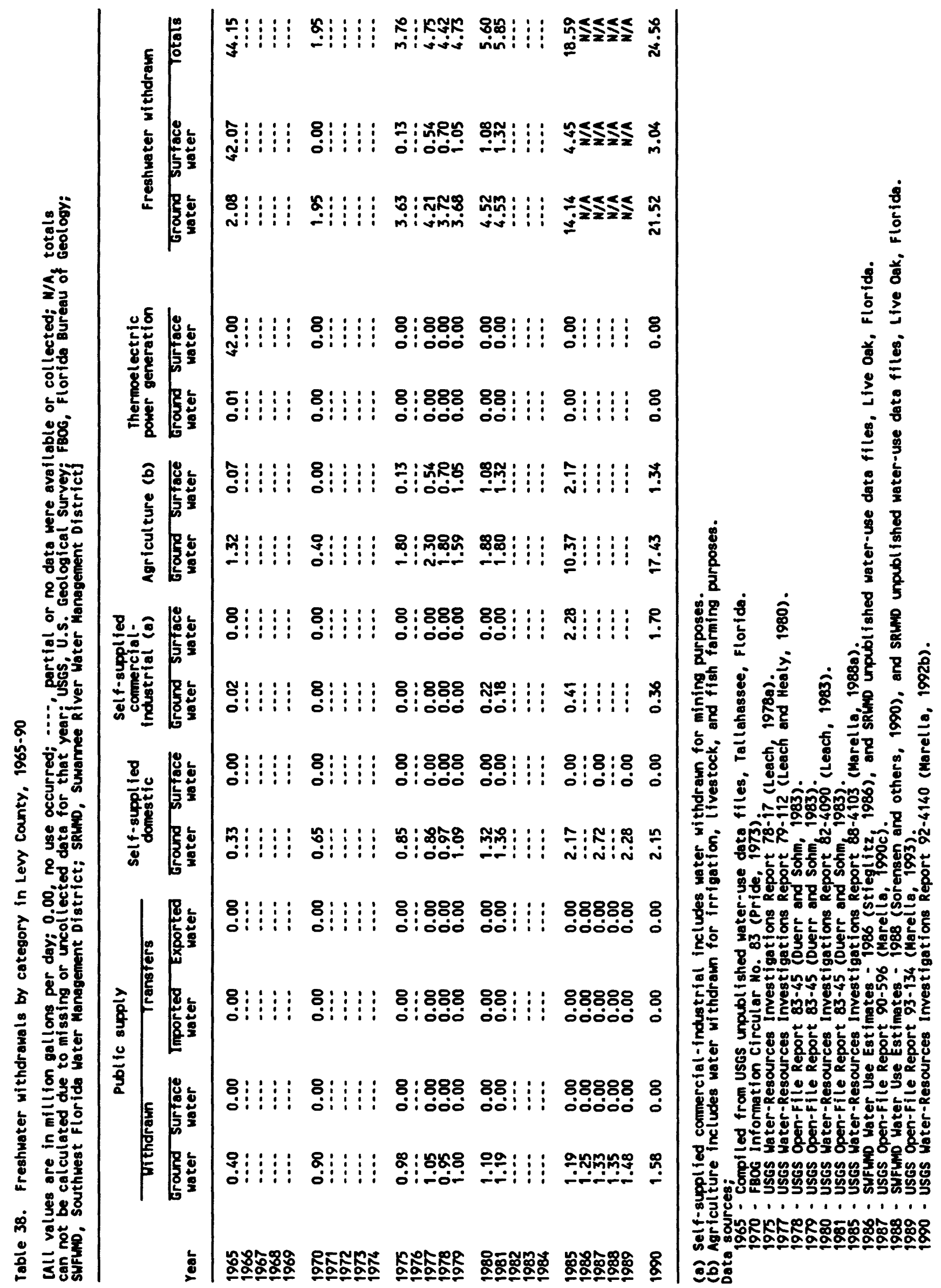




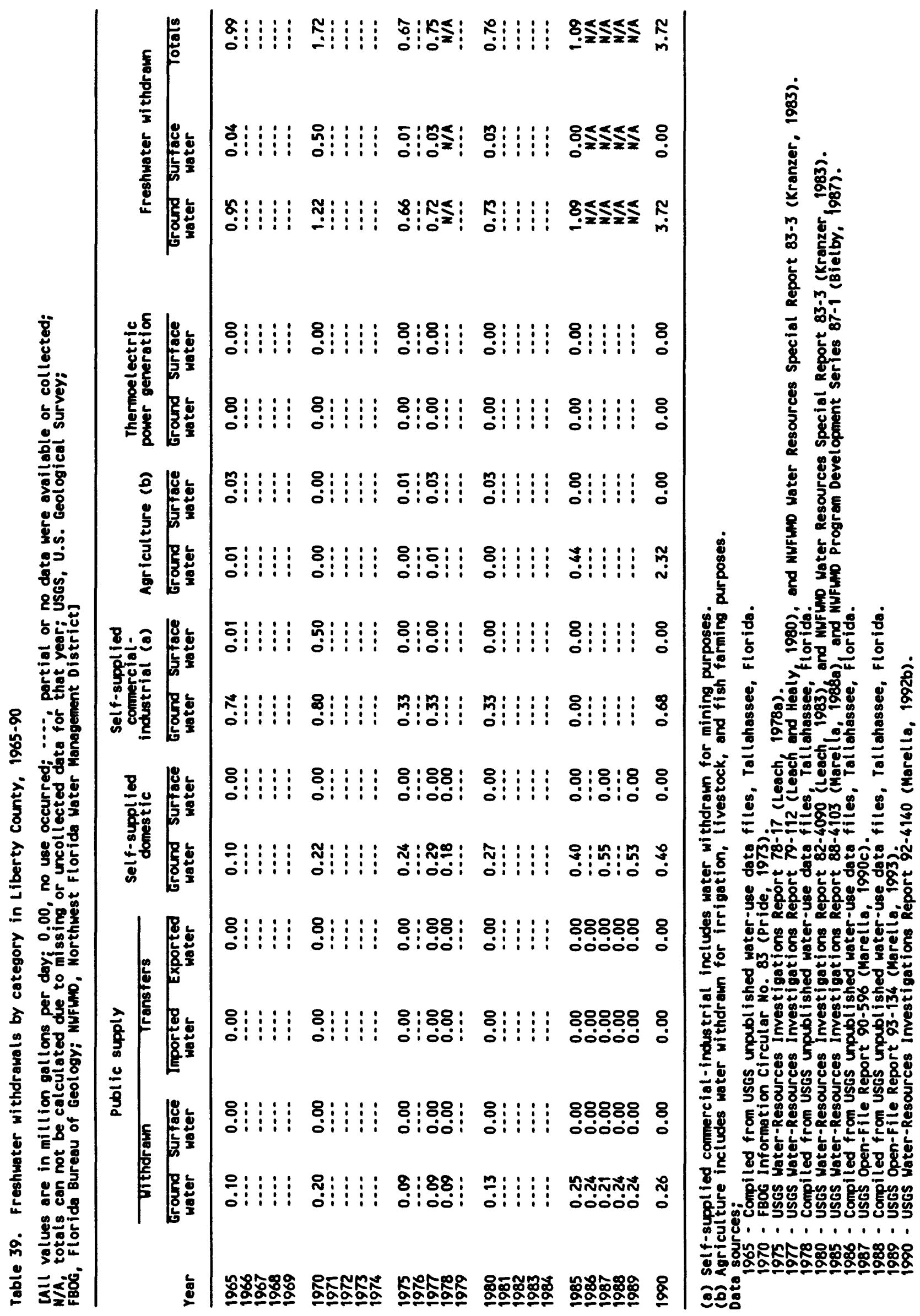




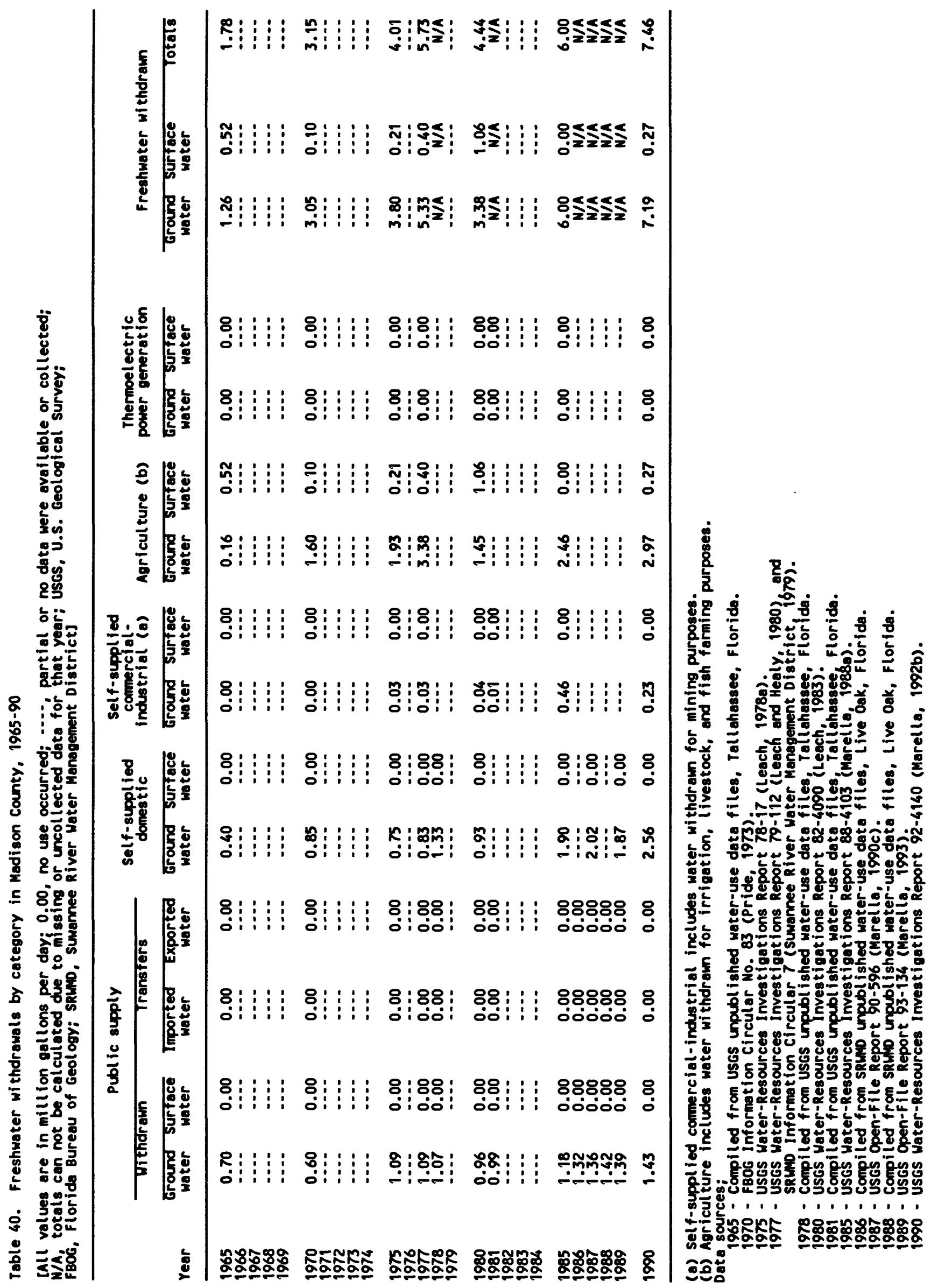




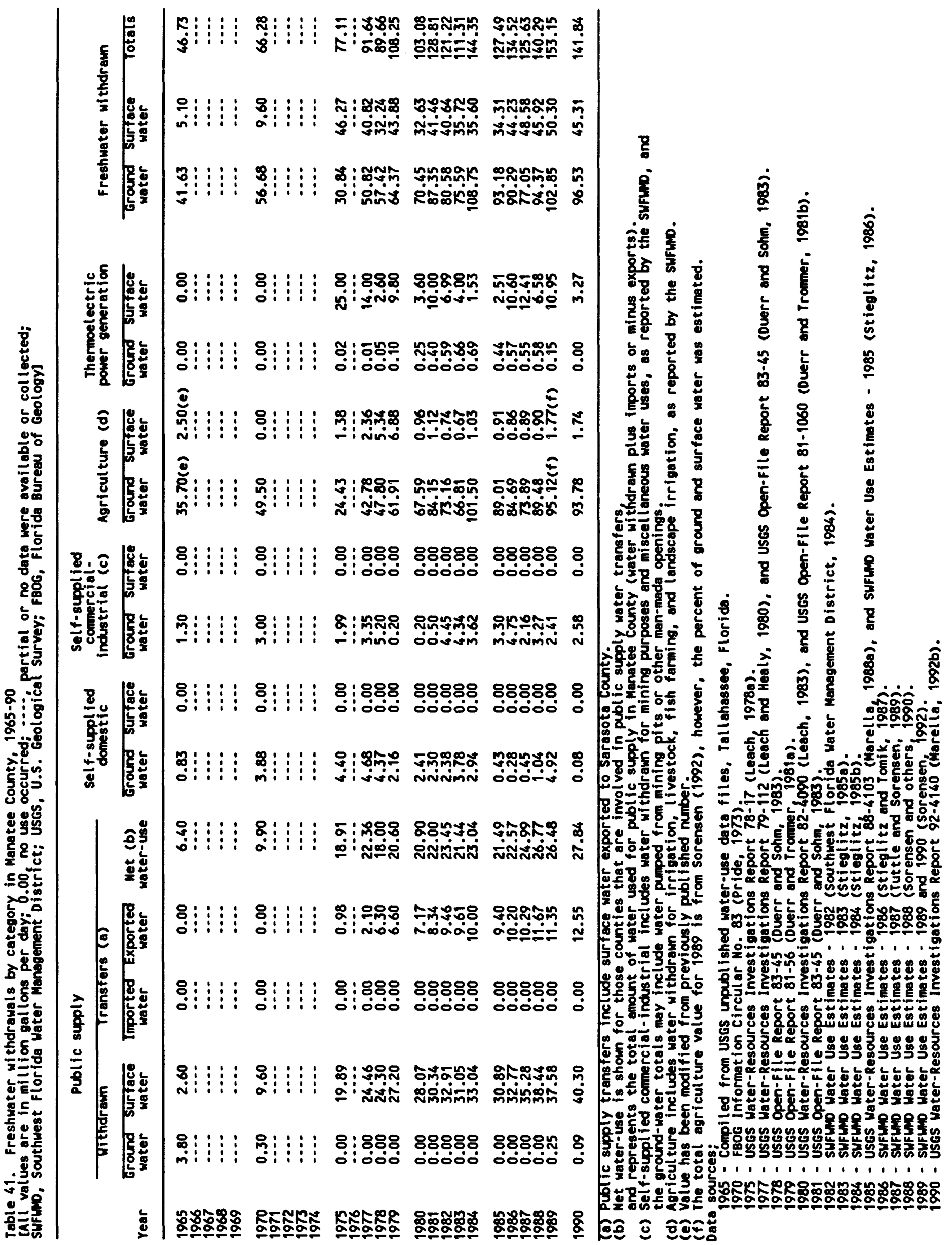




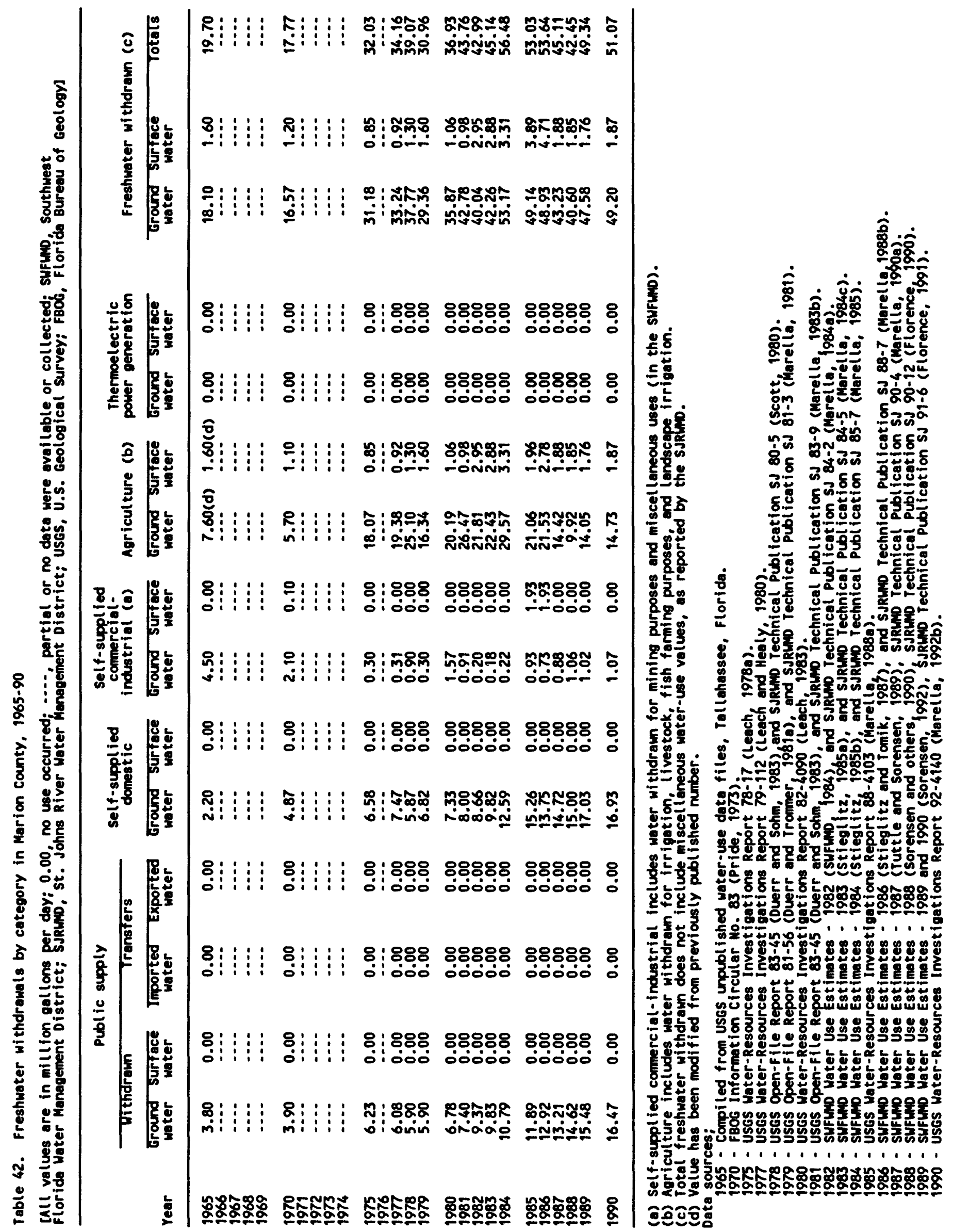




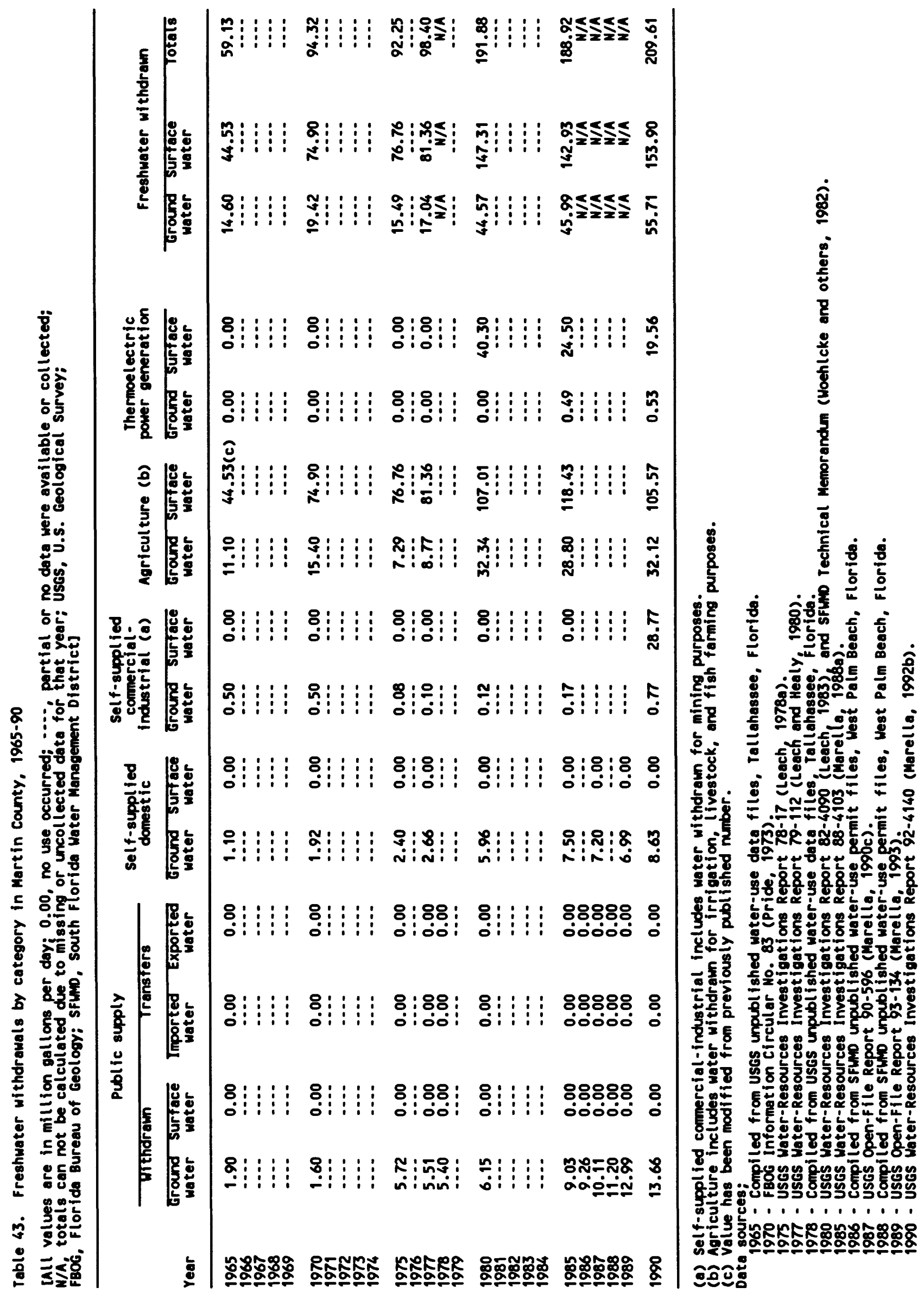




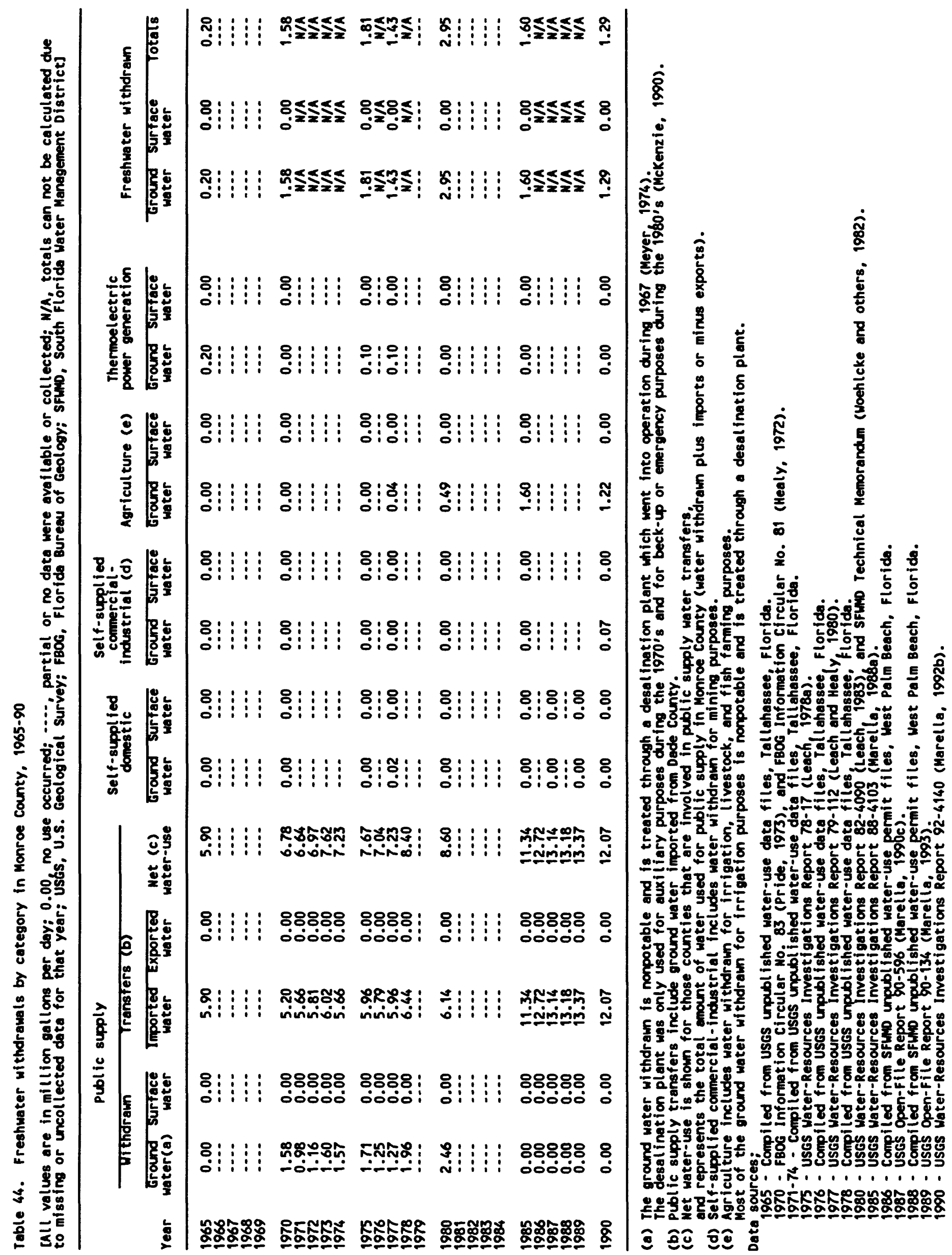




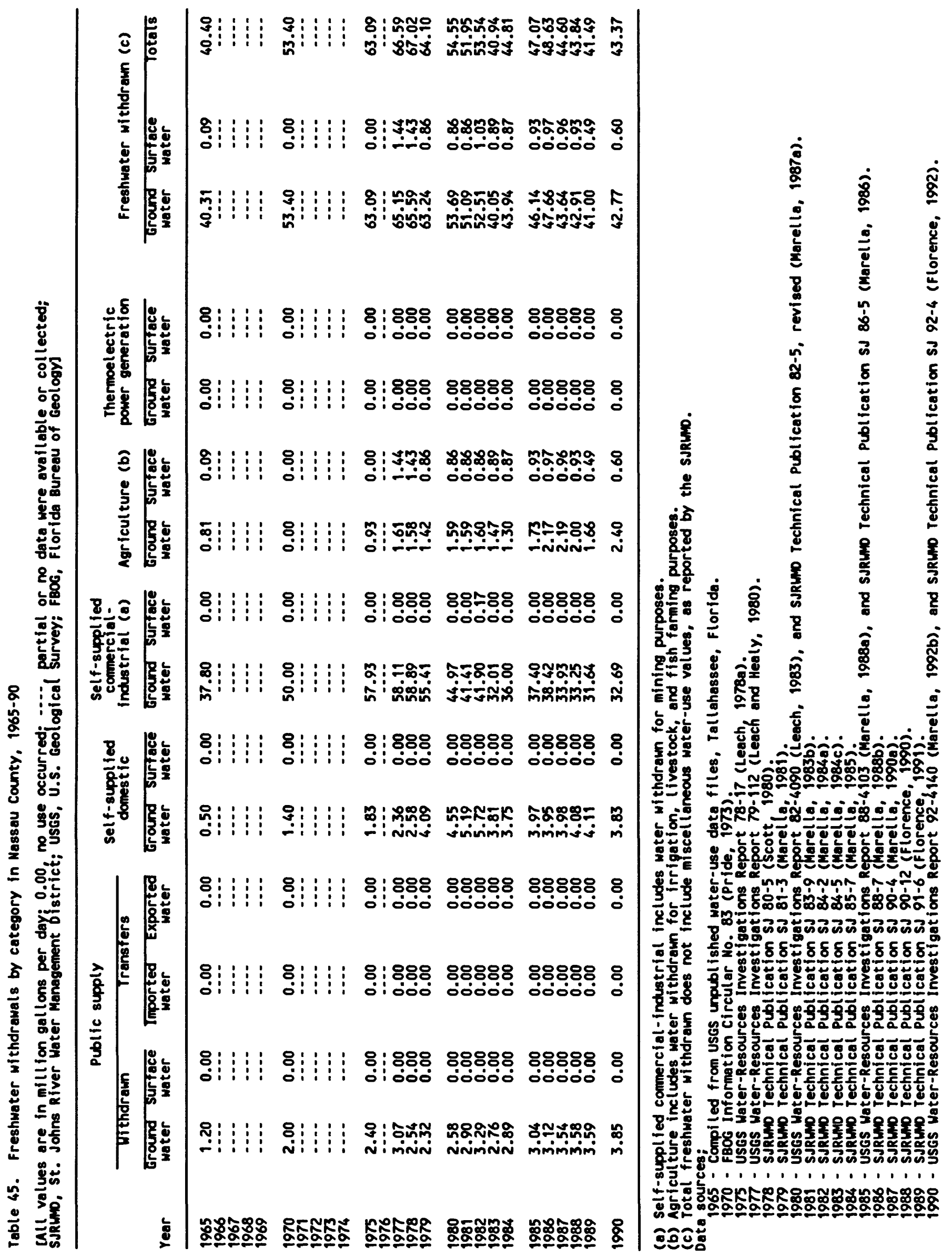




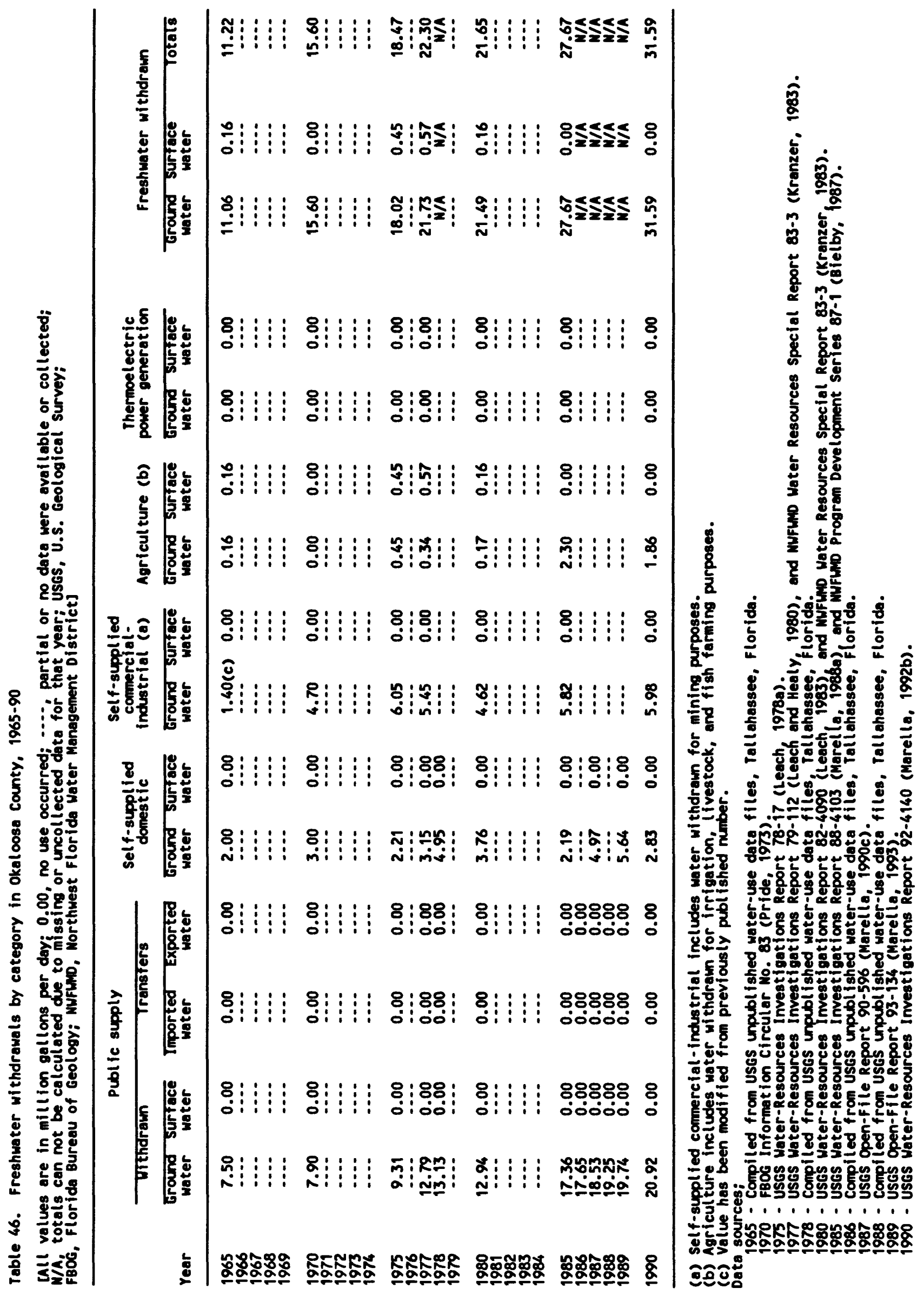




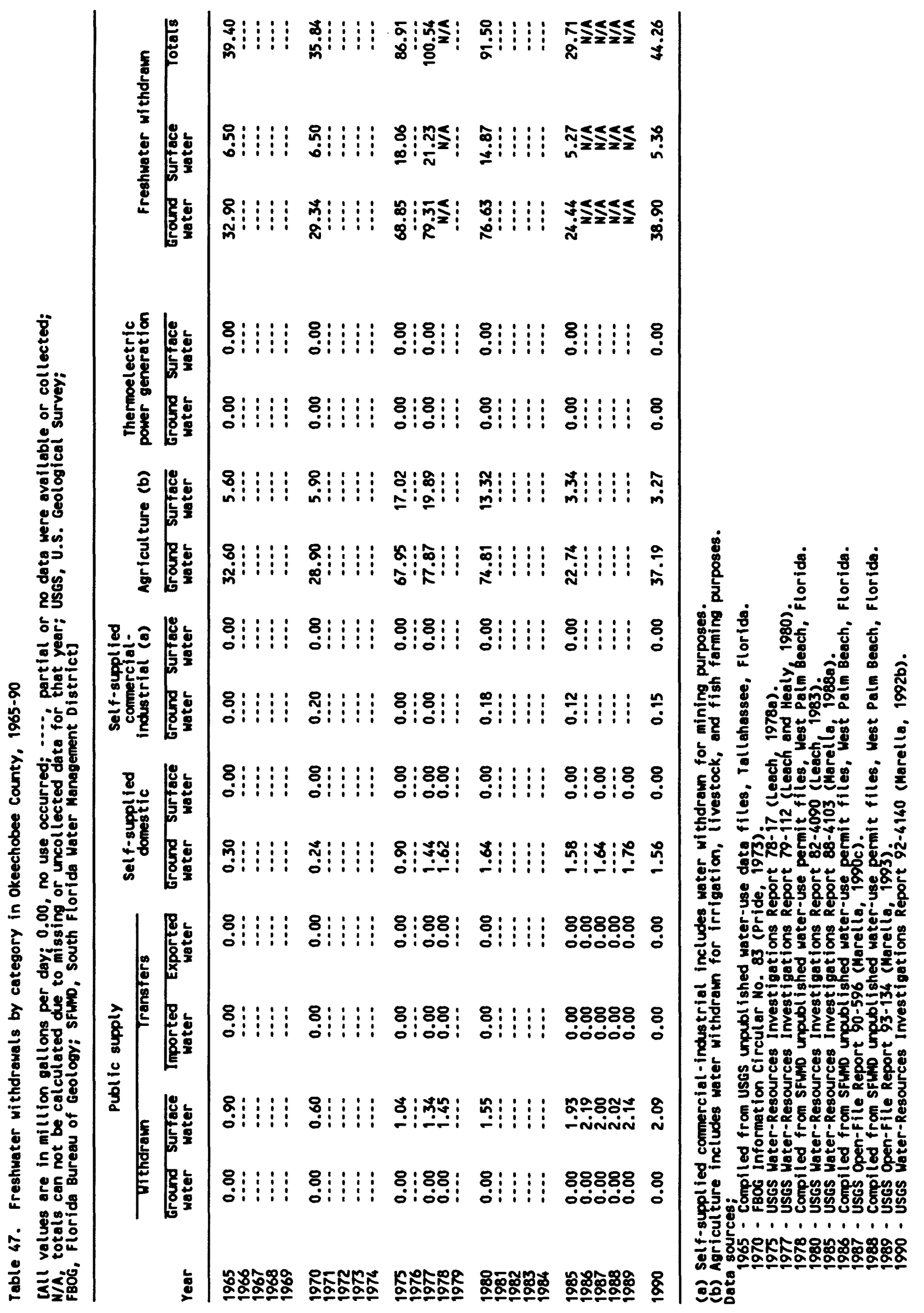




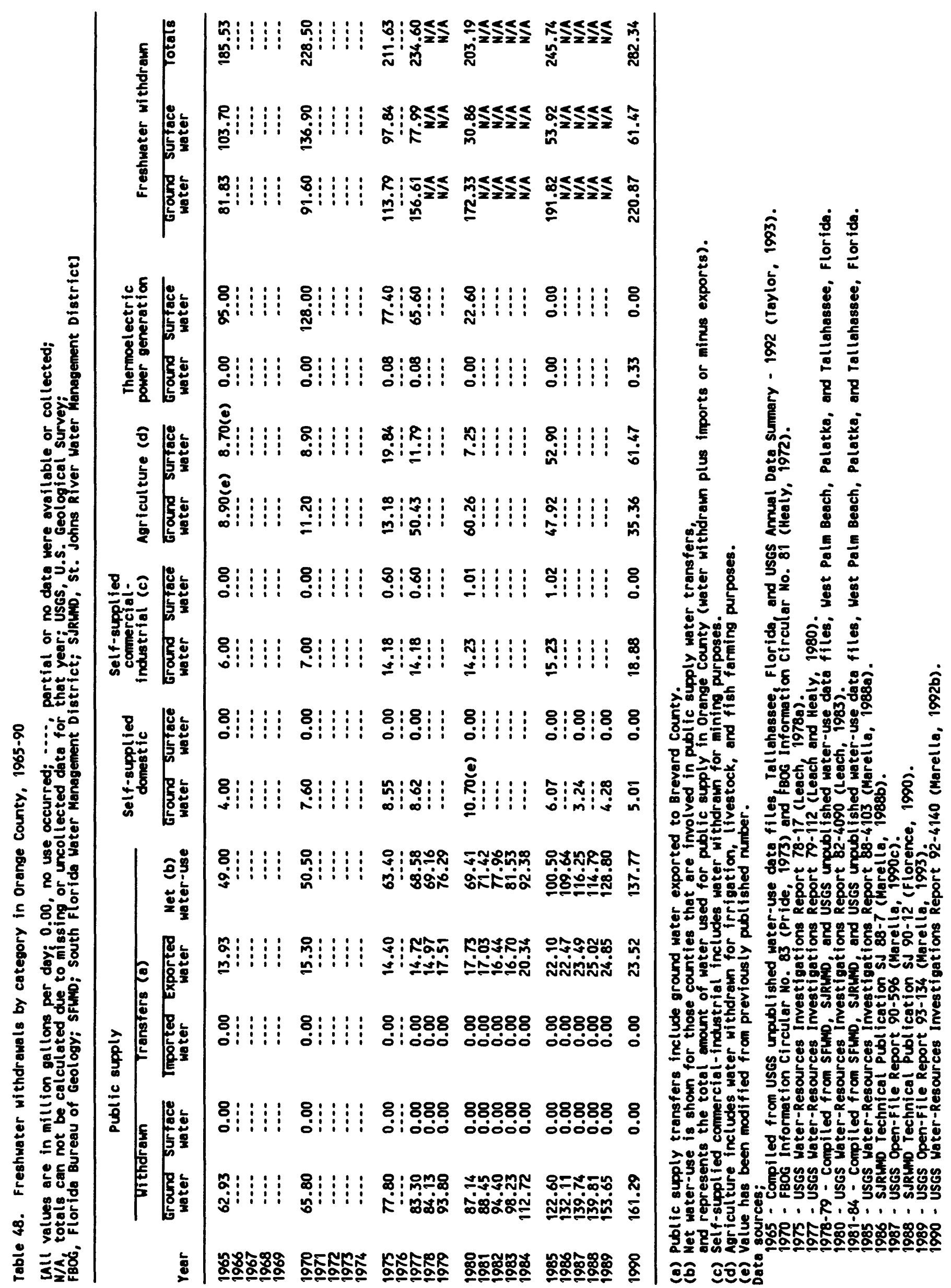




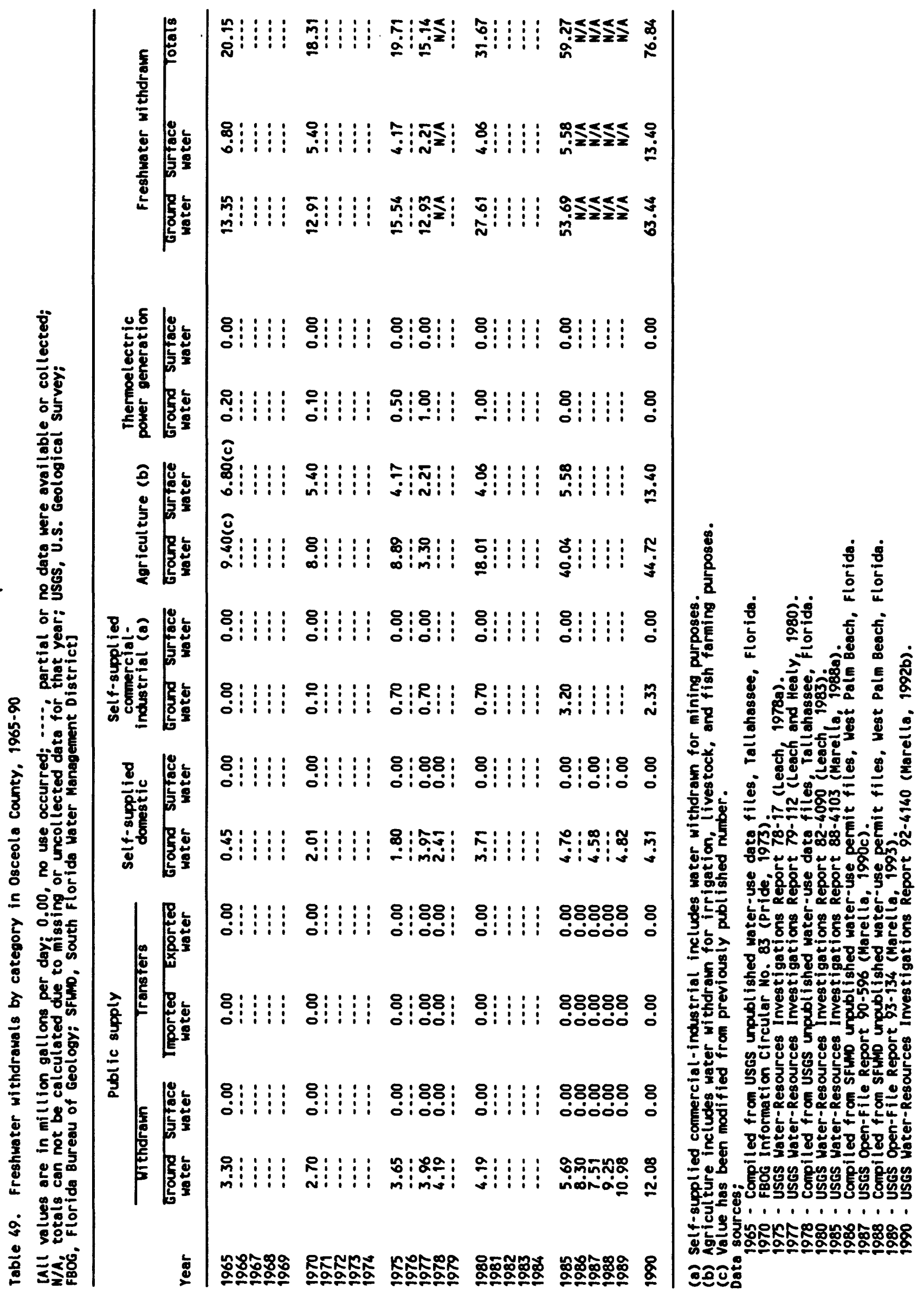




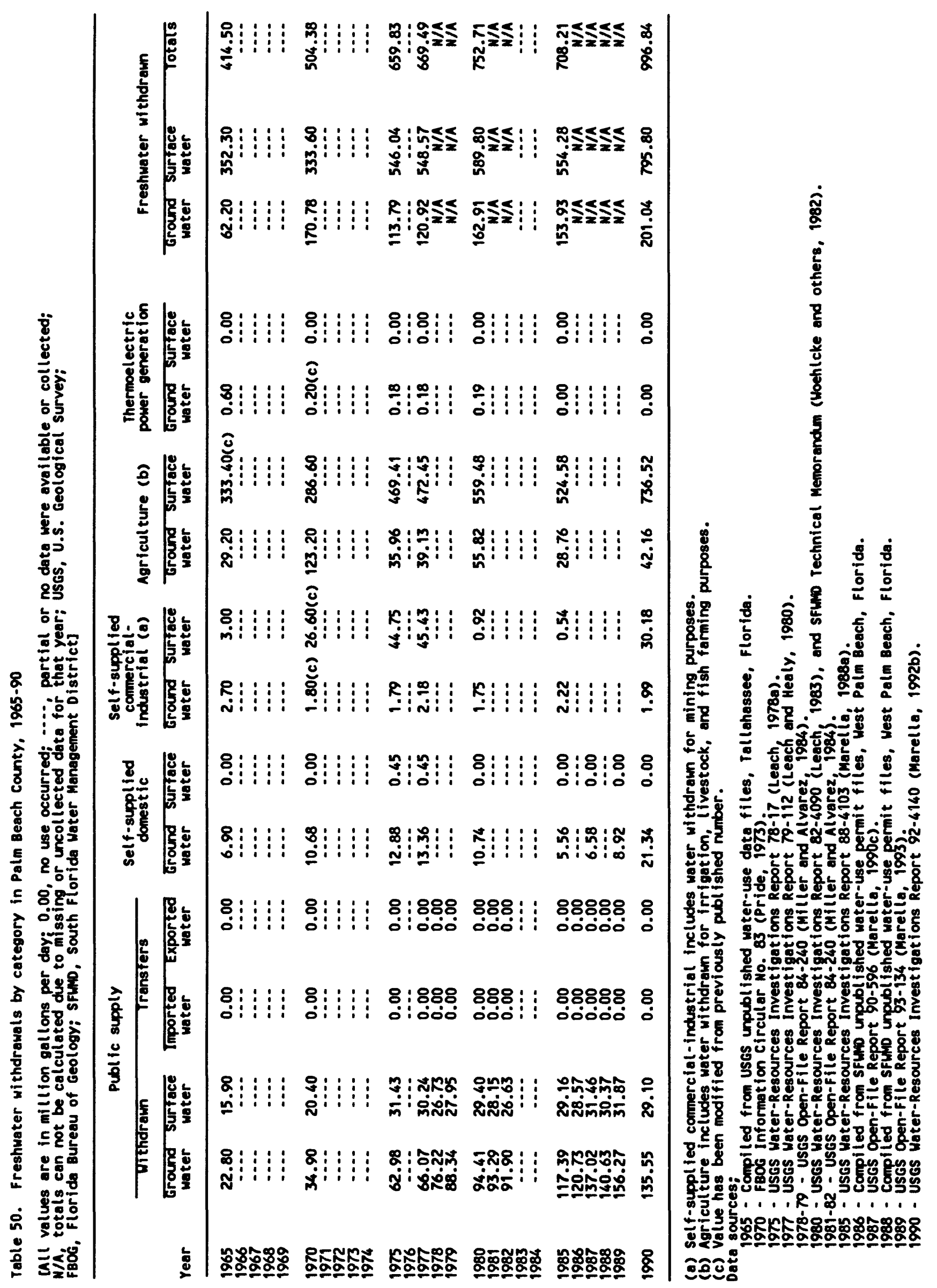




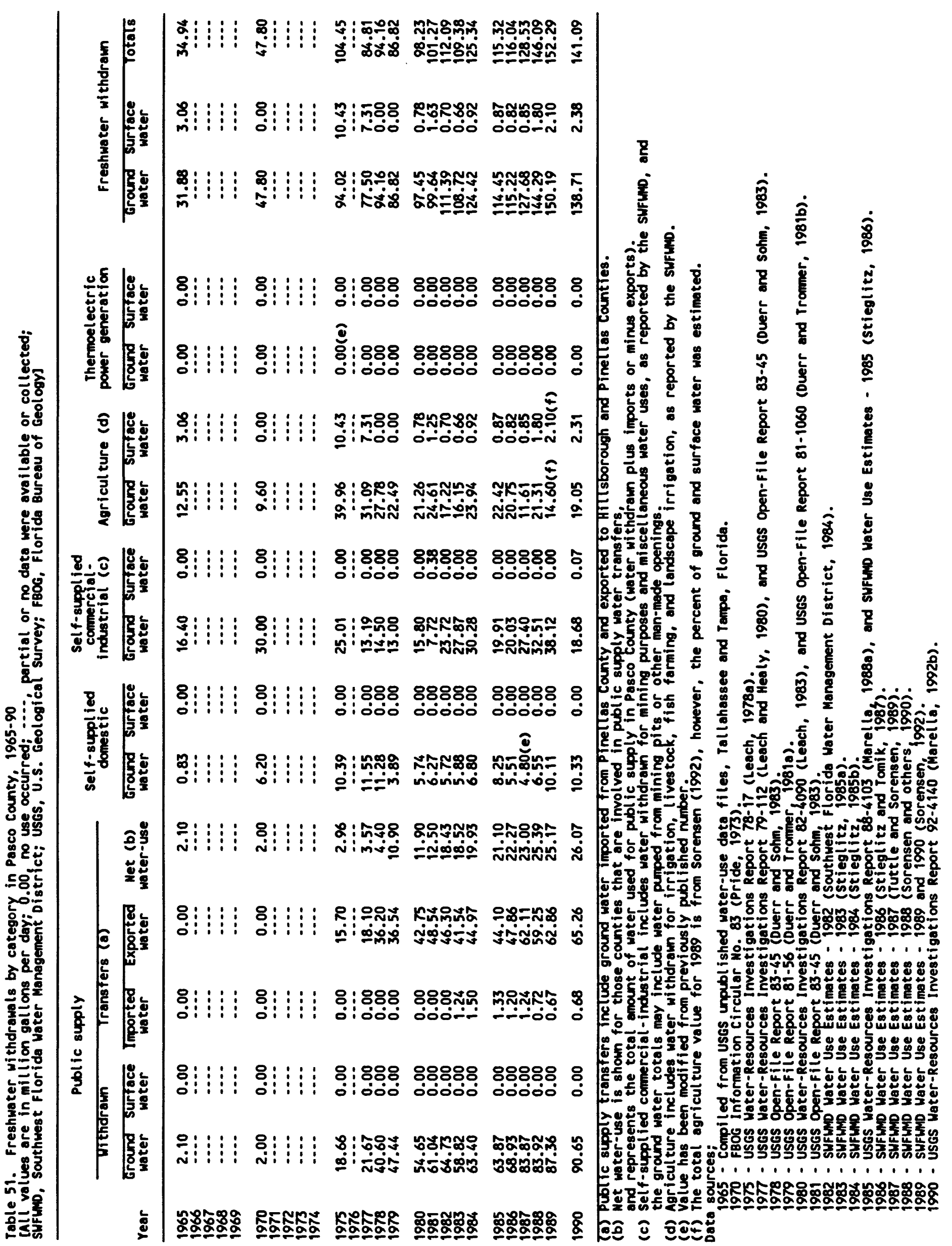




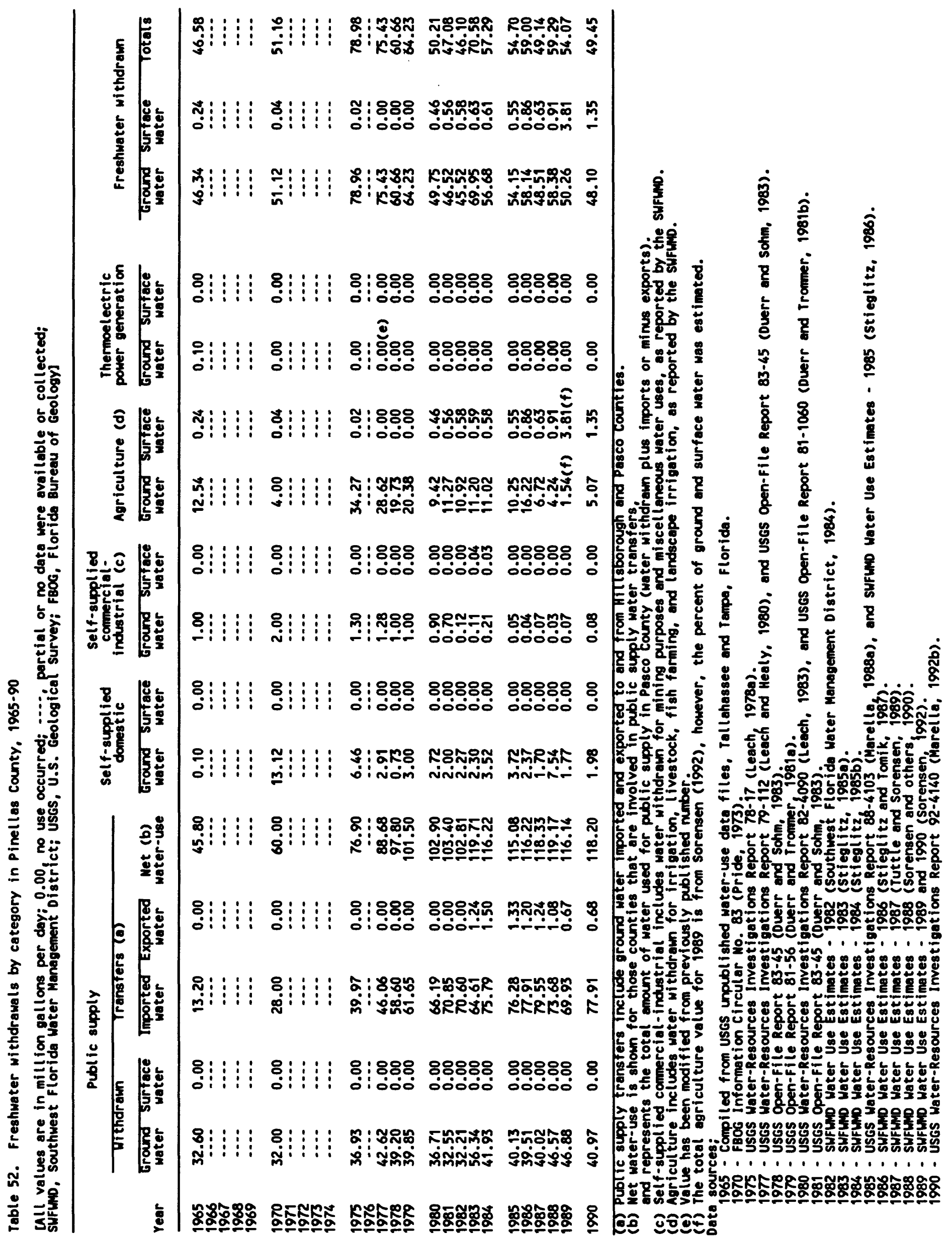




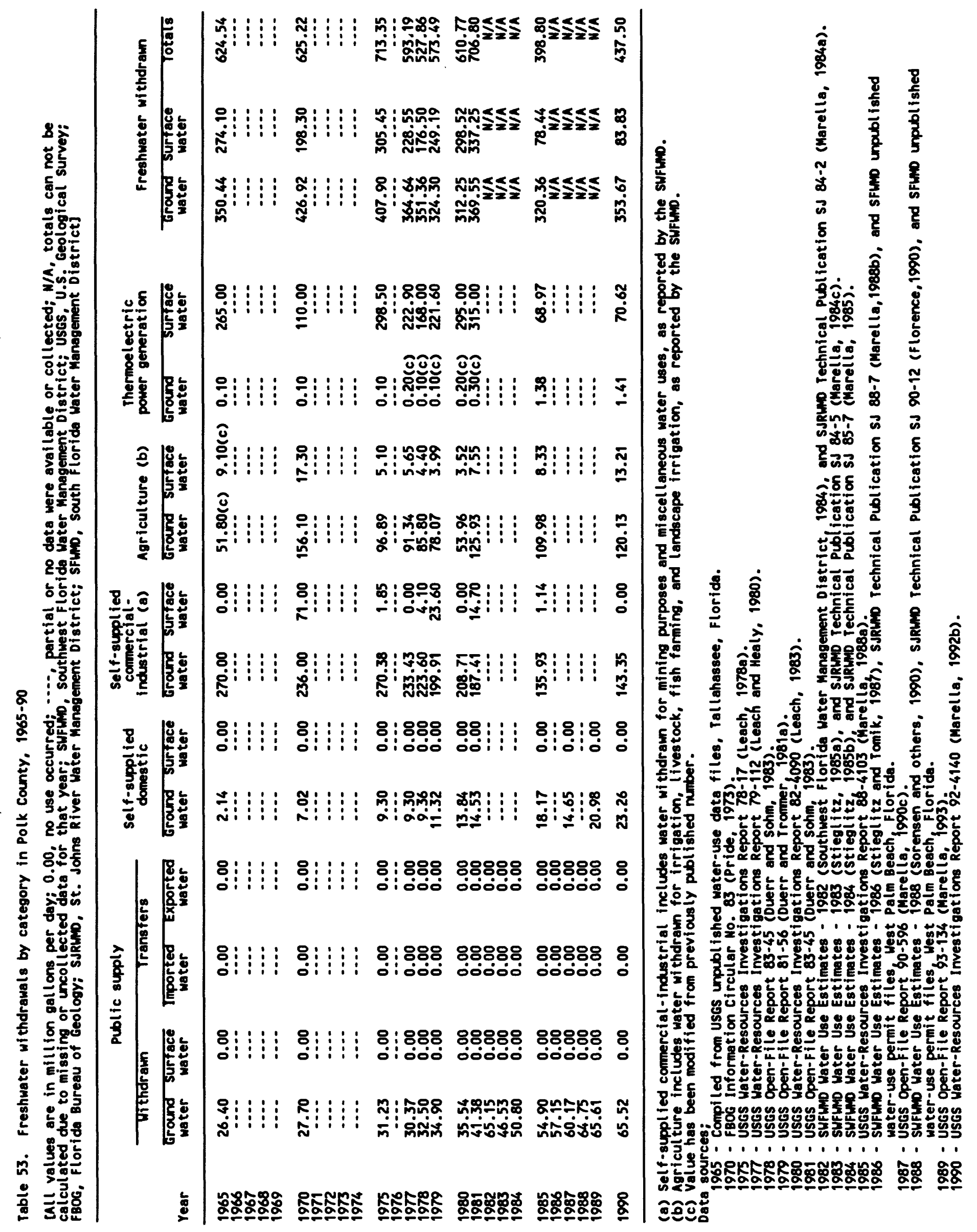




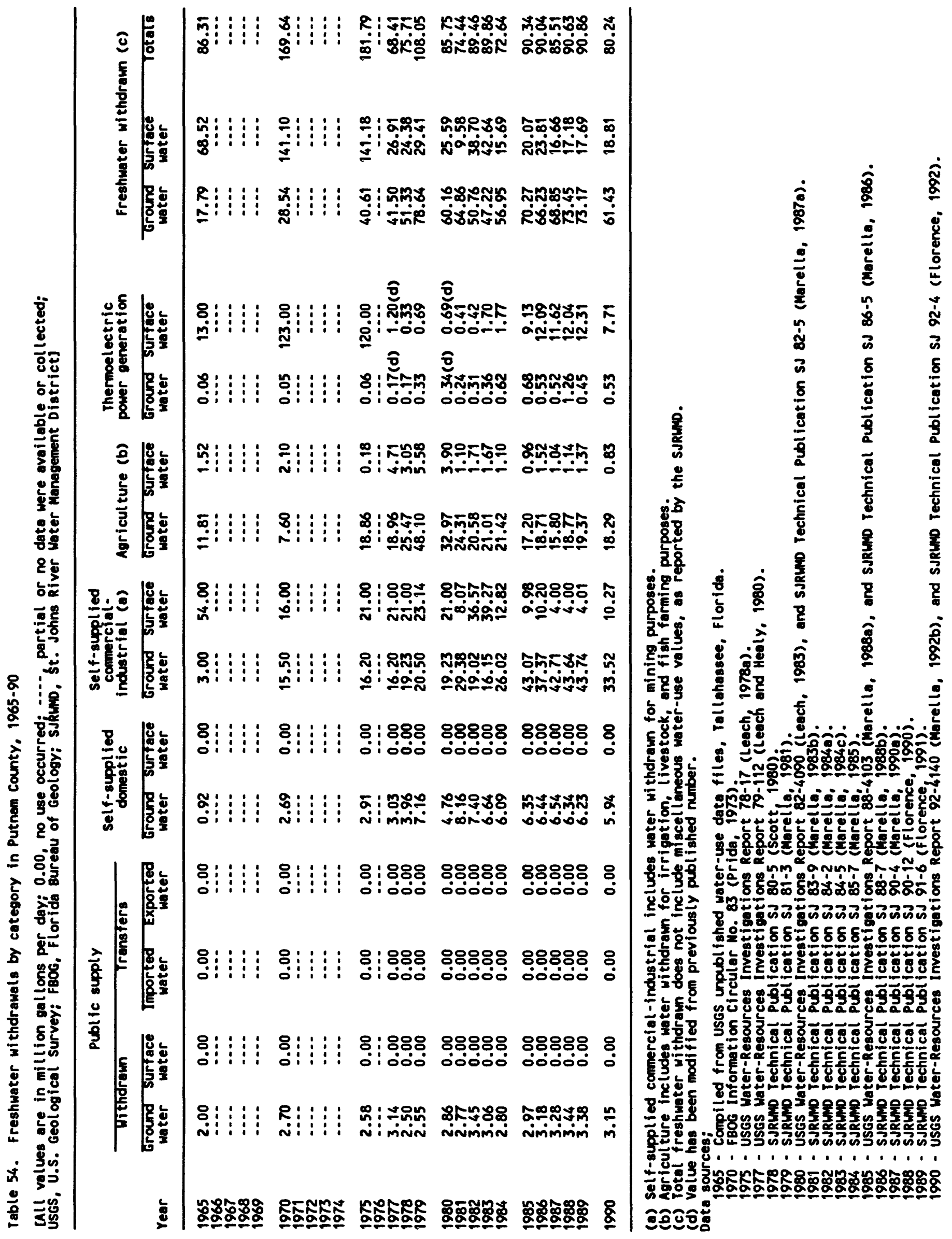




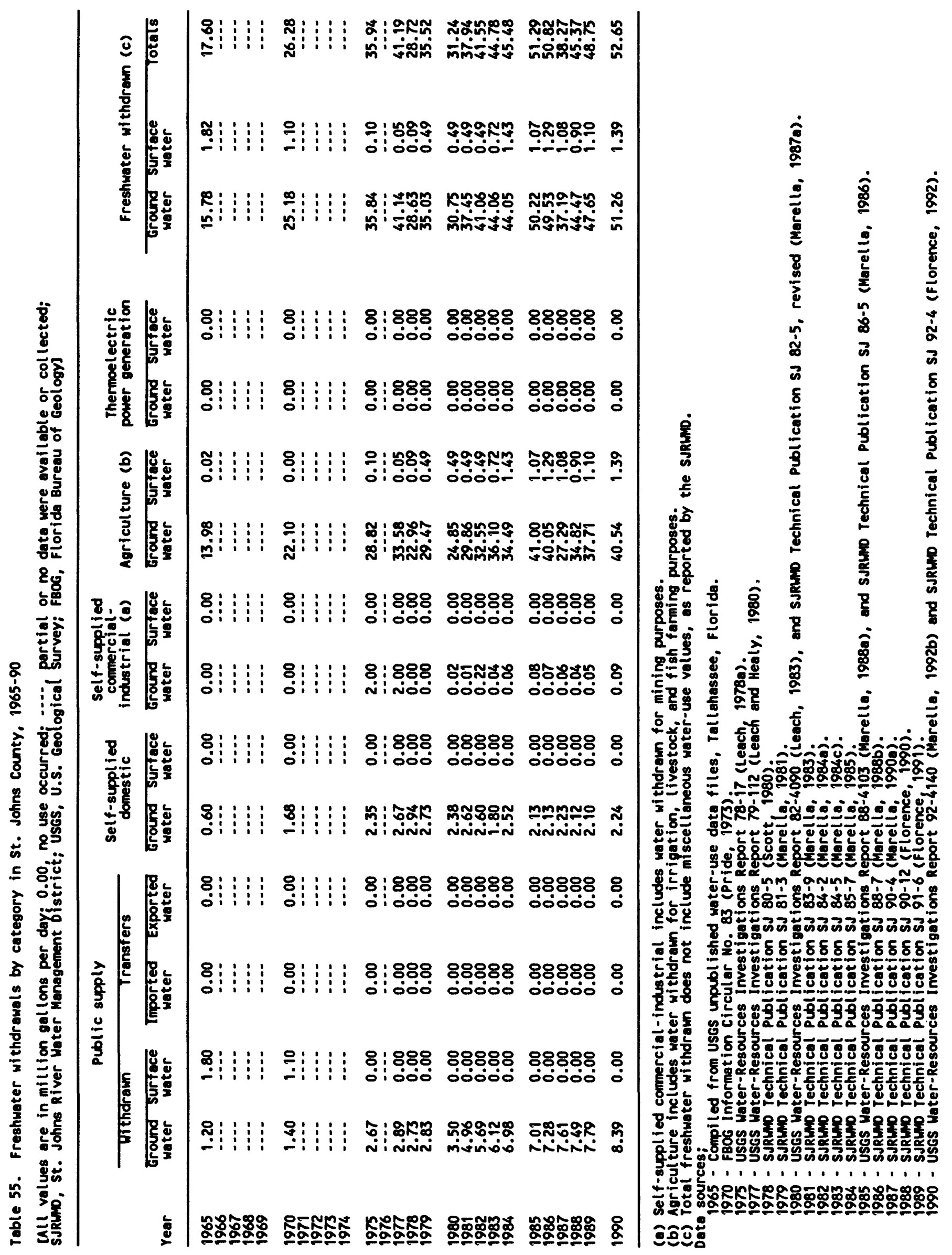




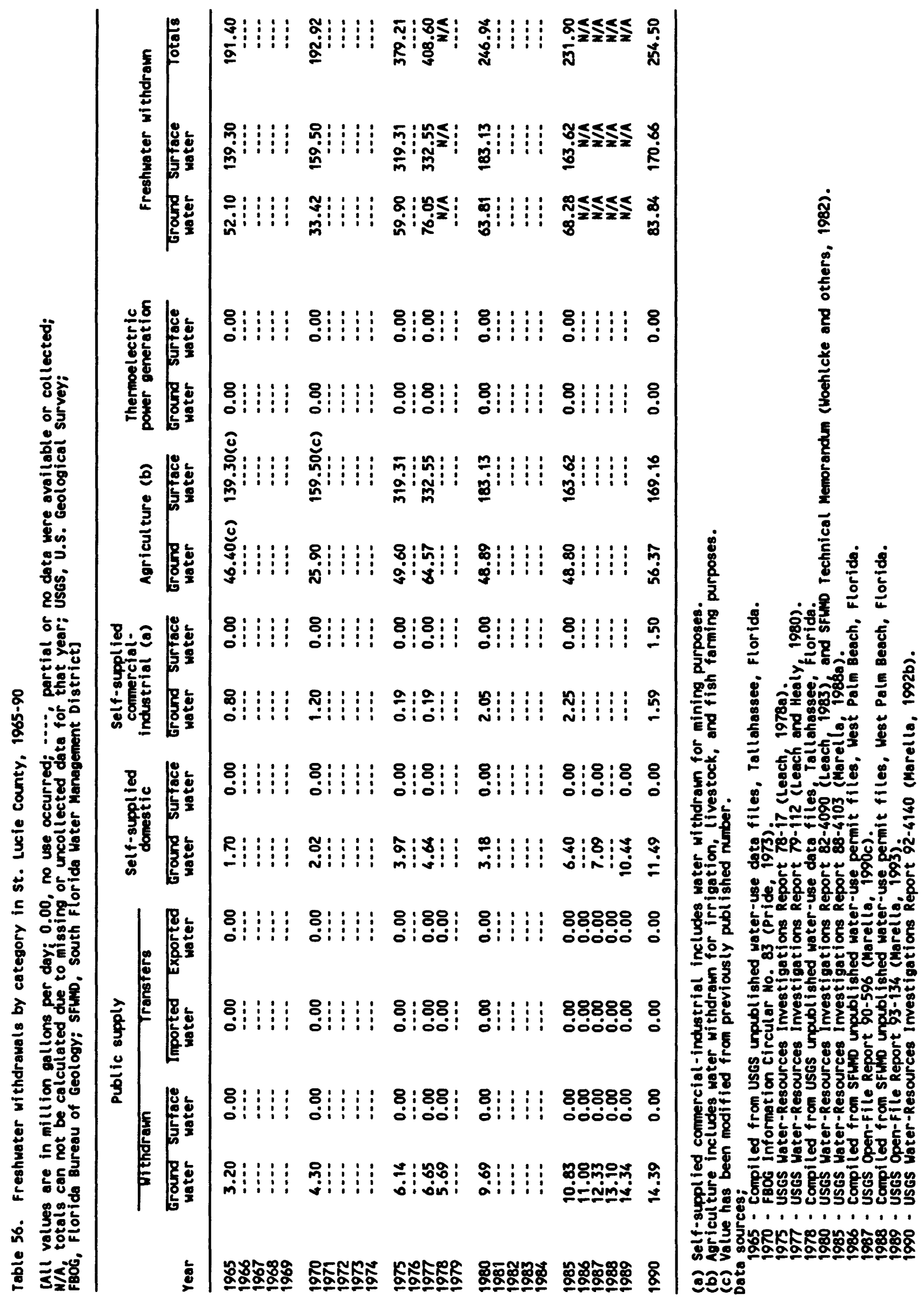




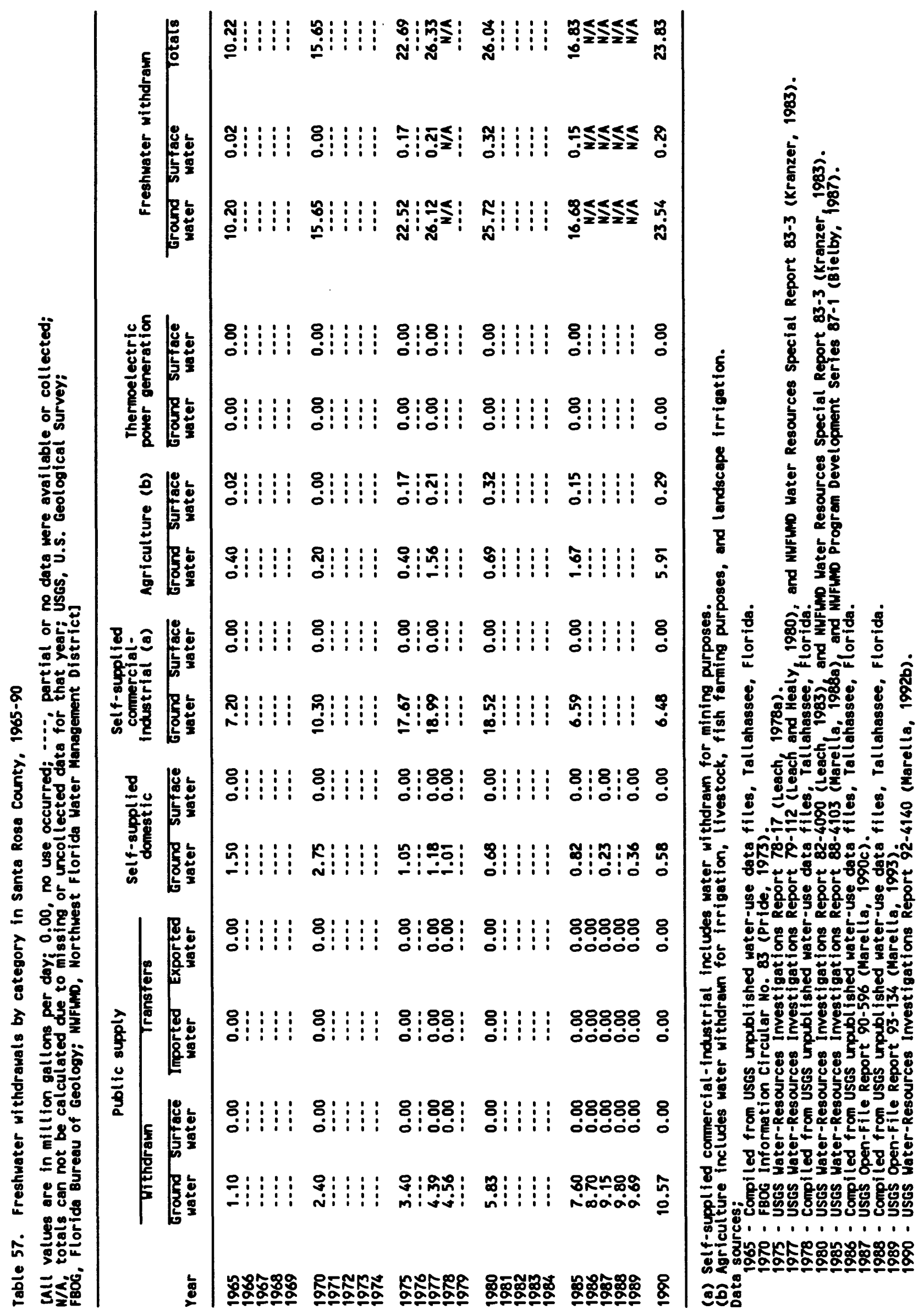




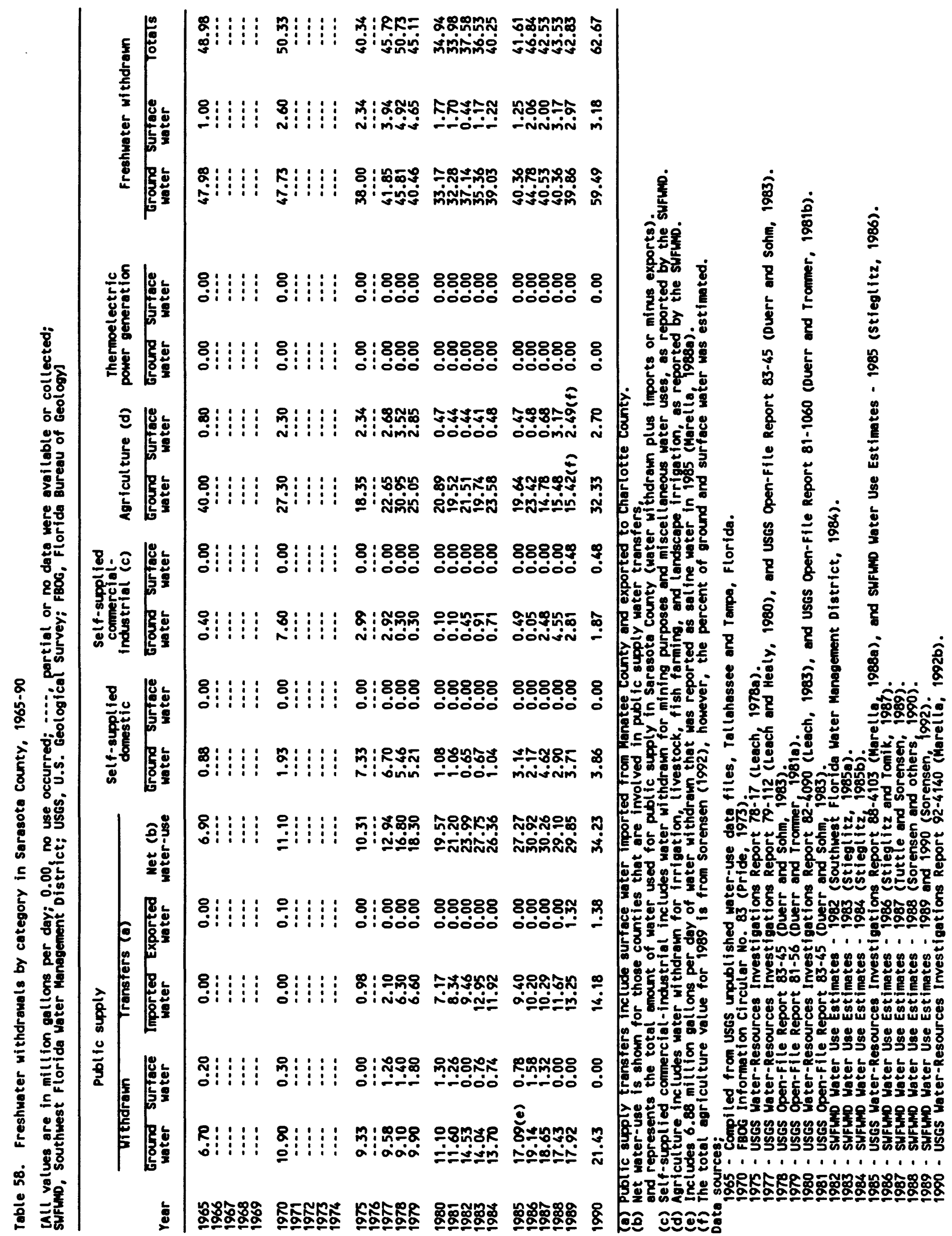




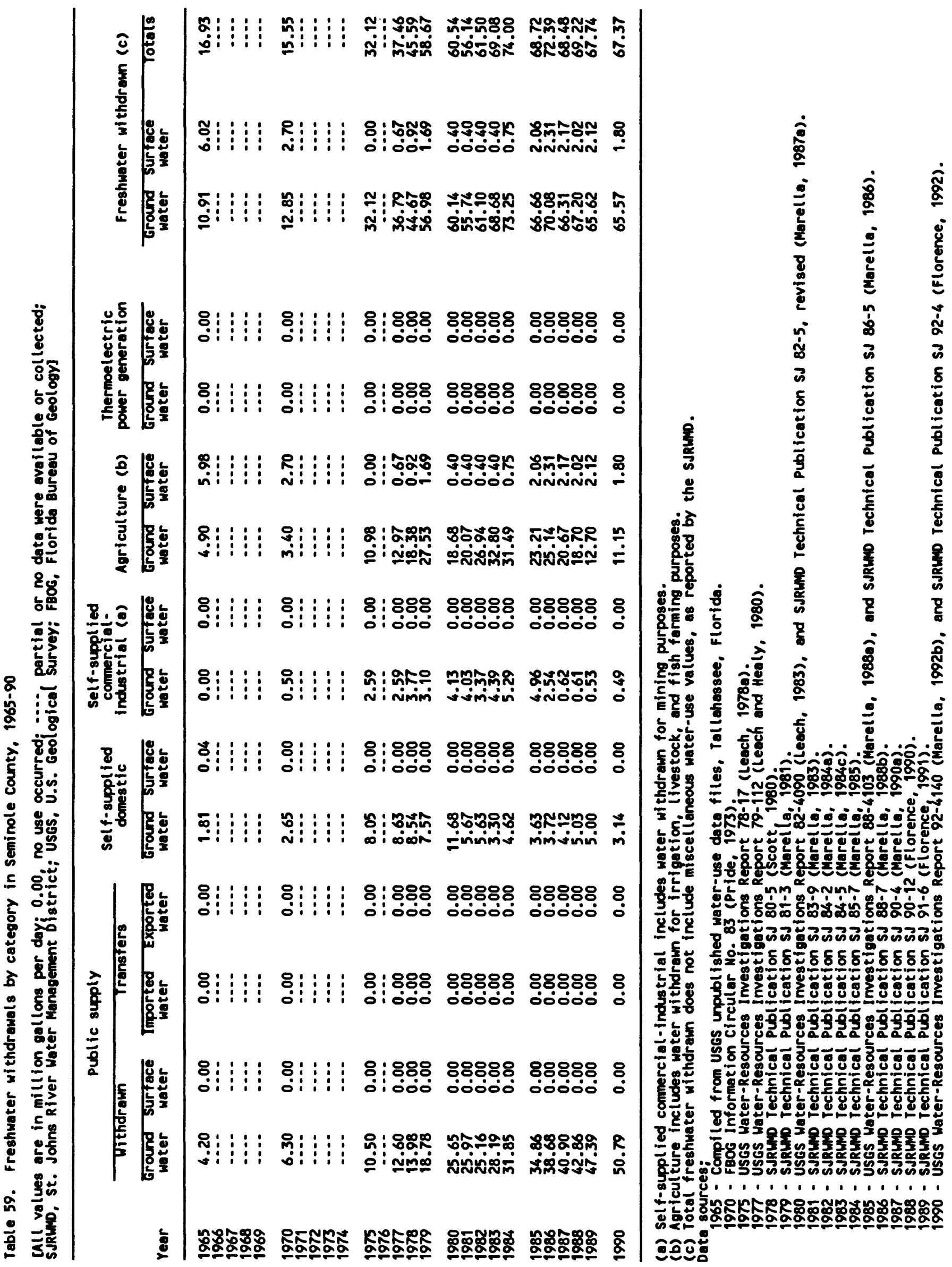




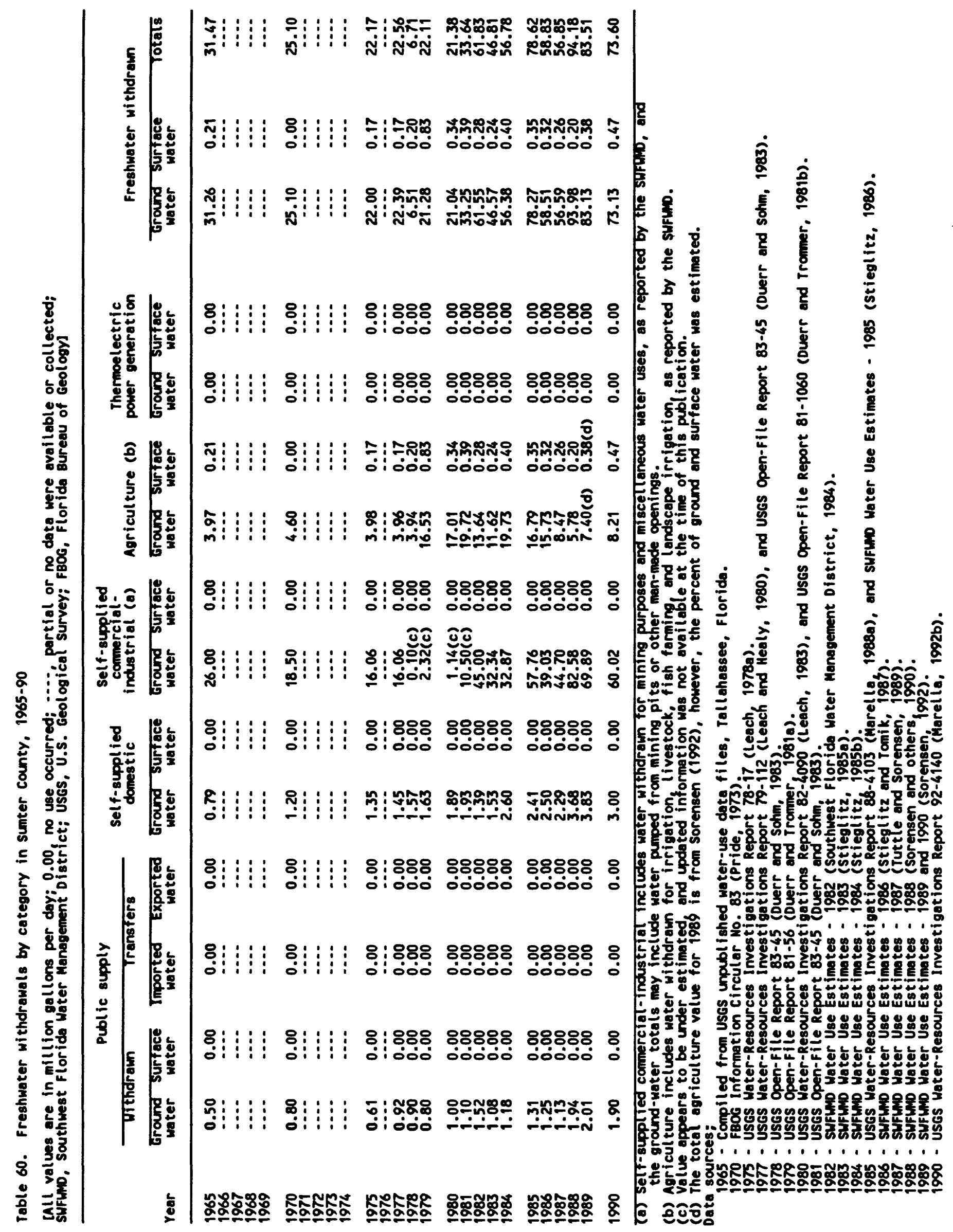

Appendix 107 


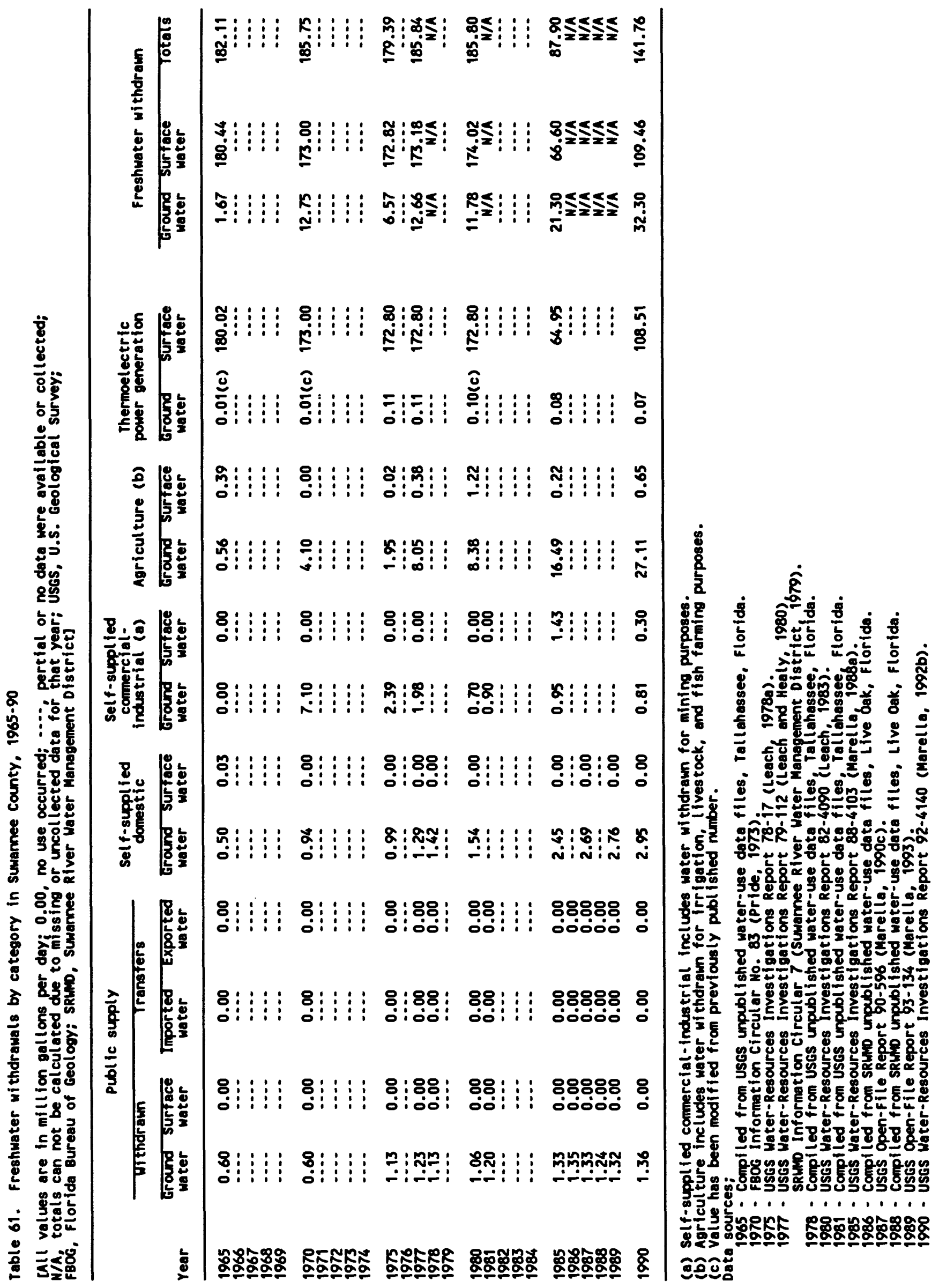




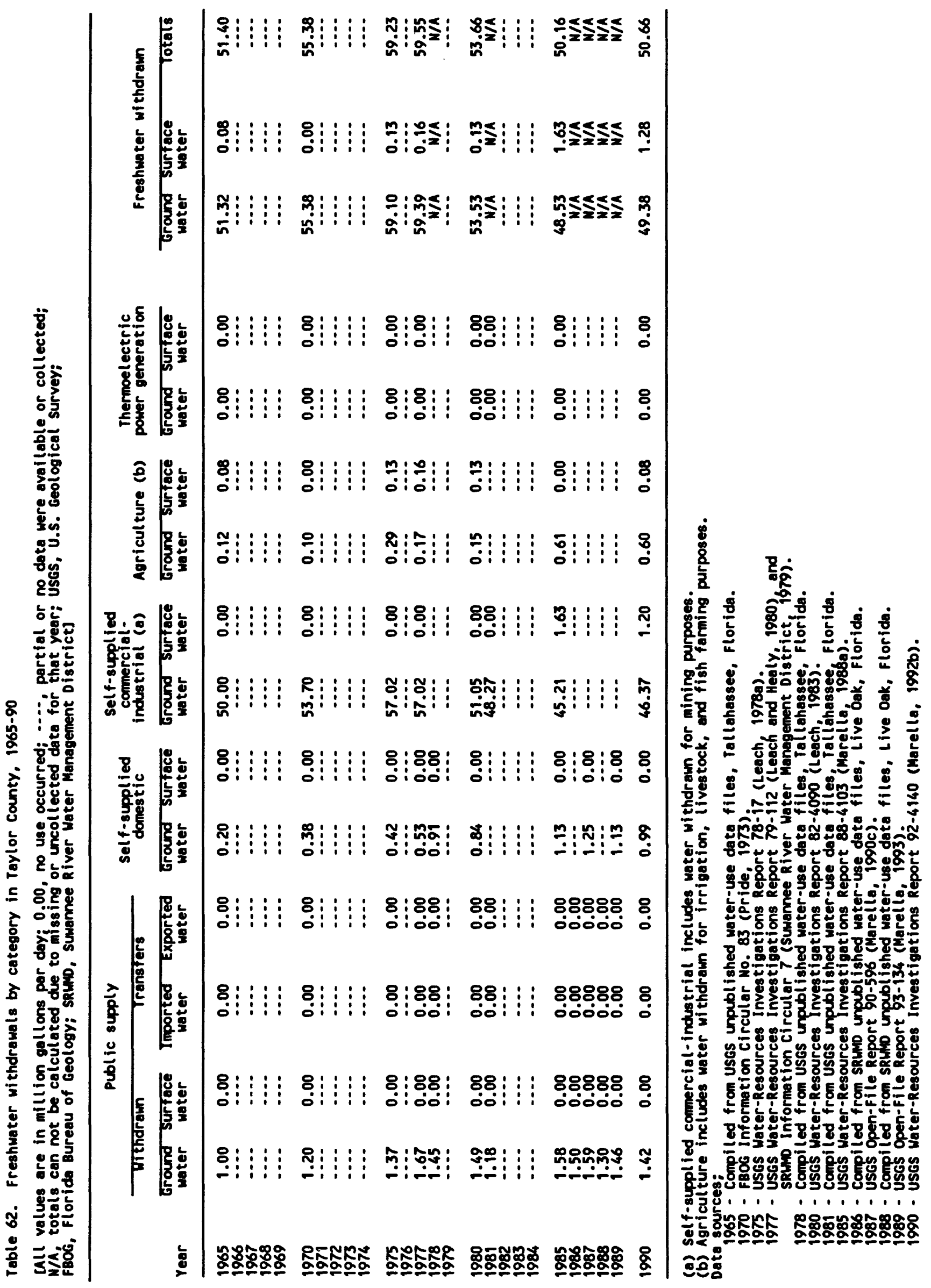




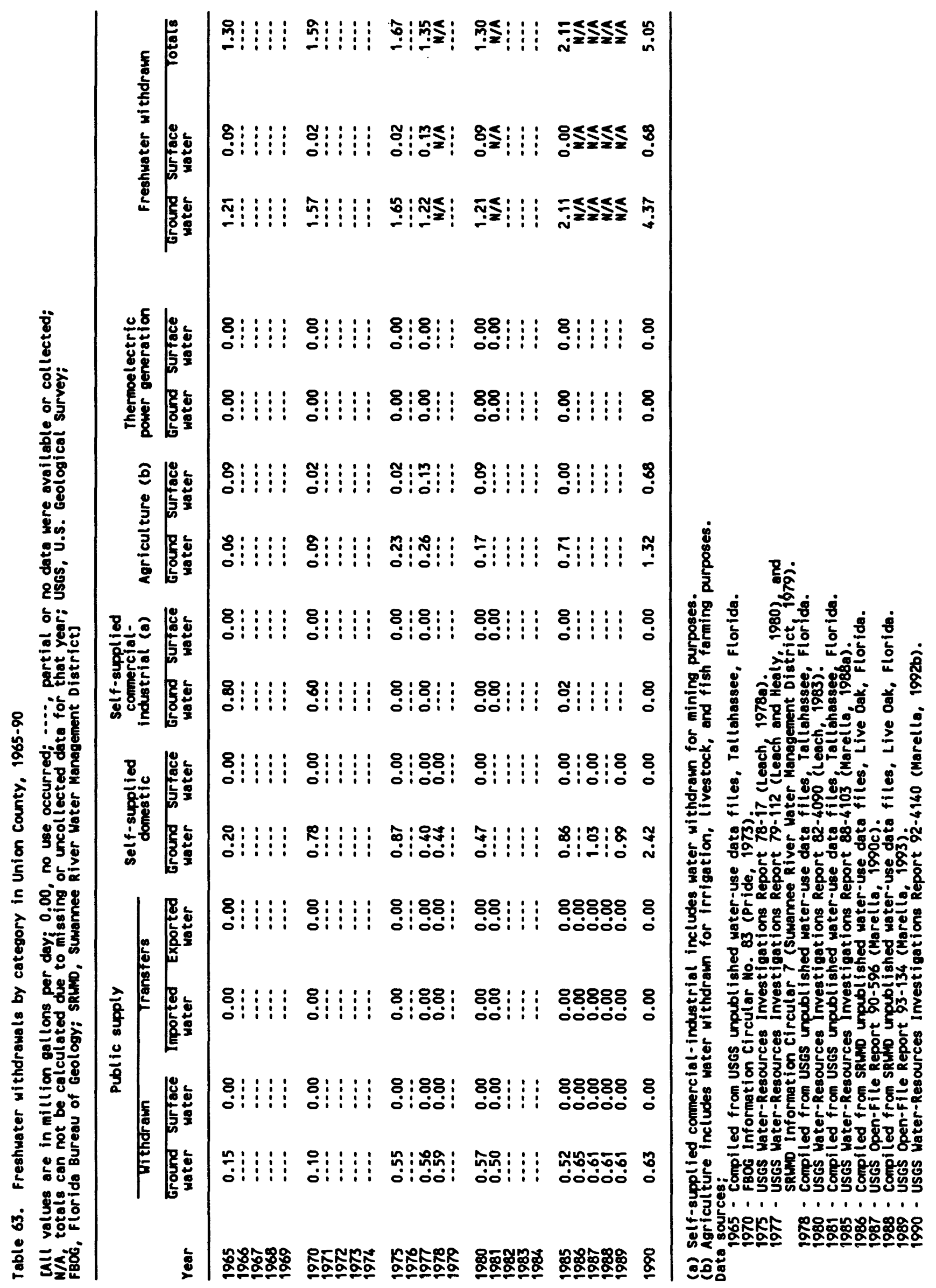




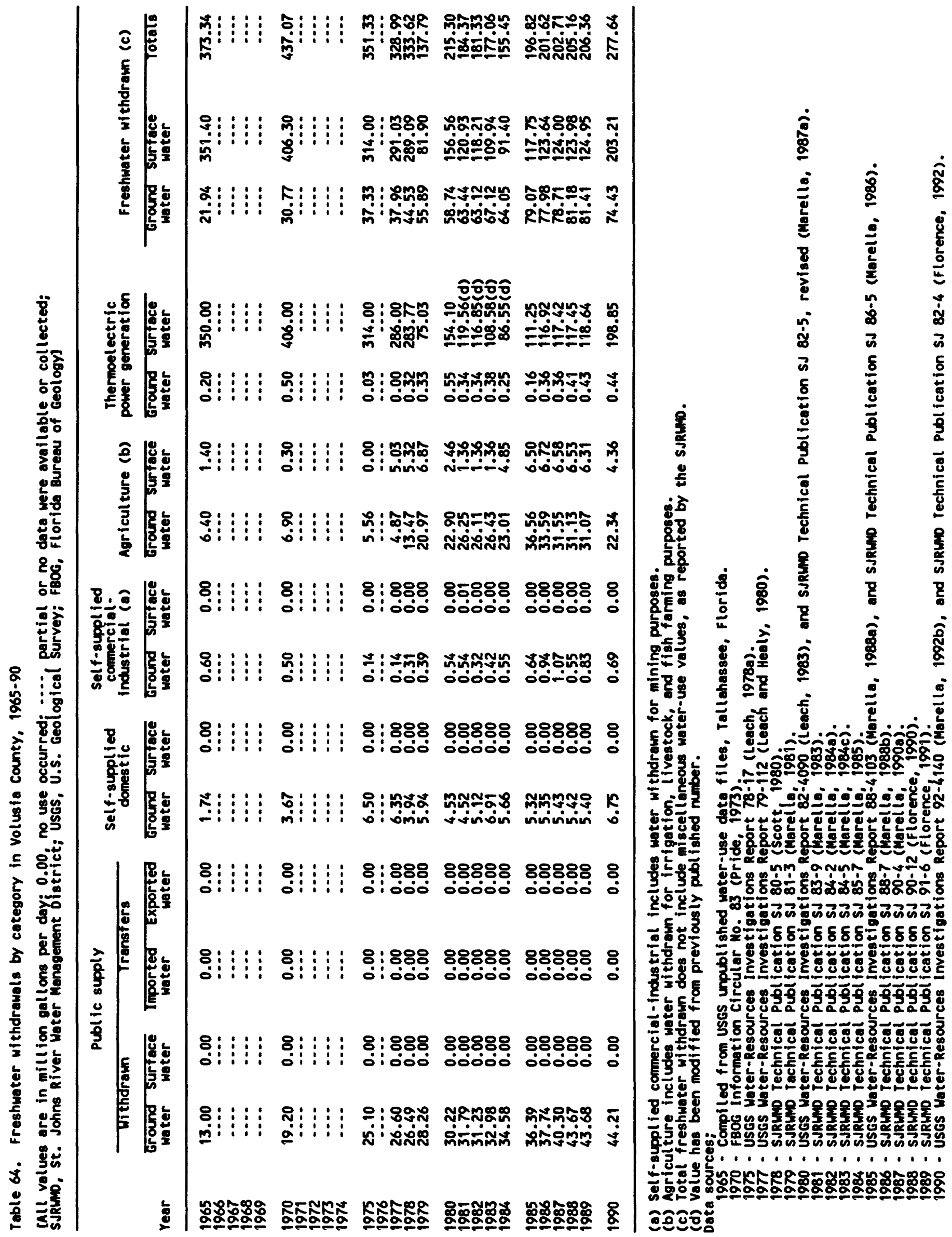




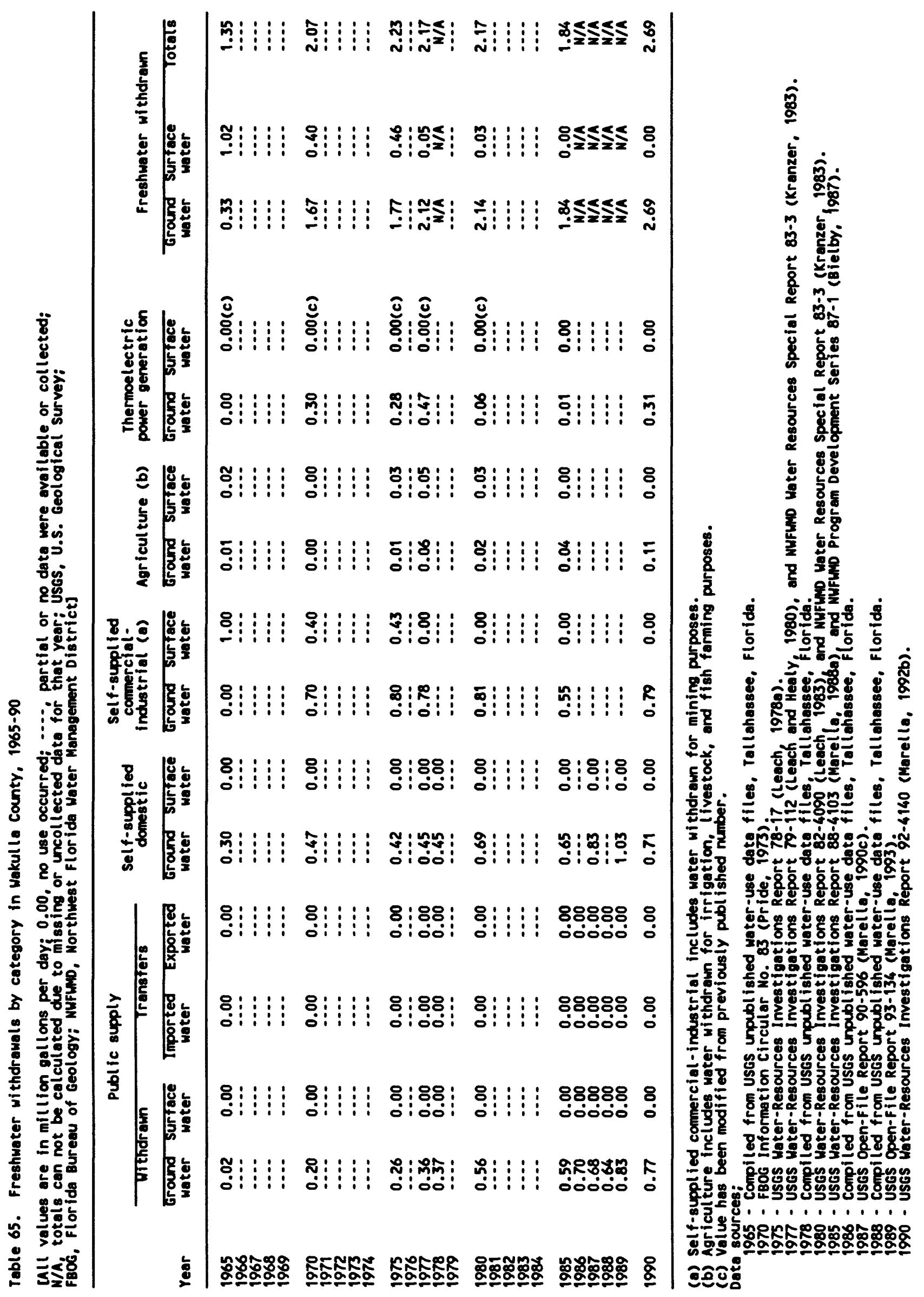




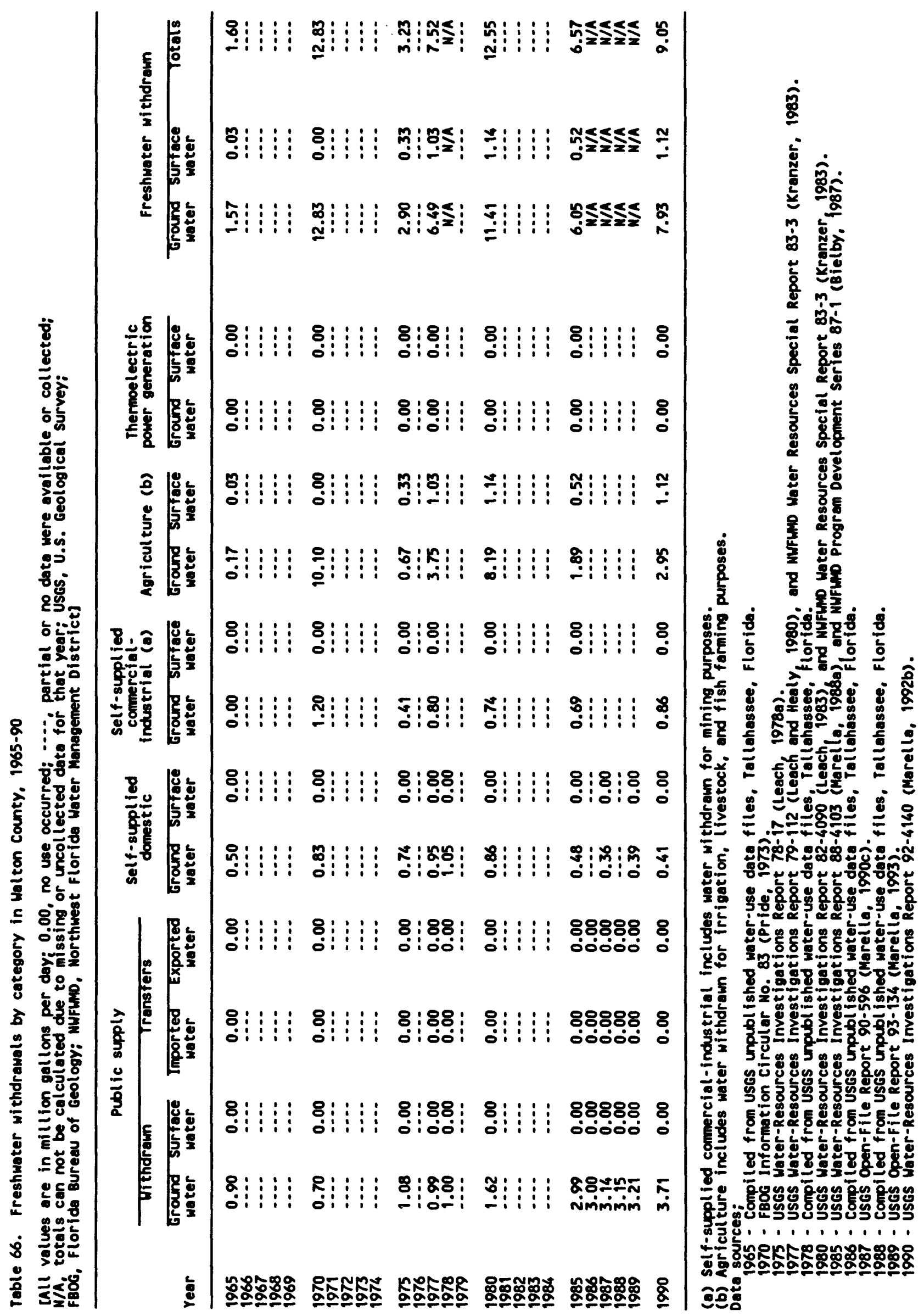




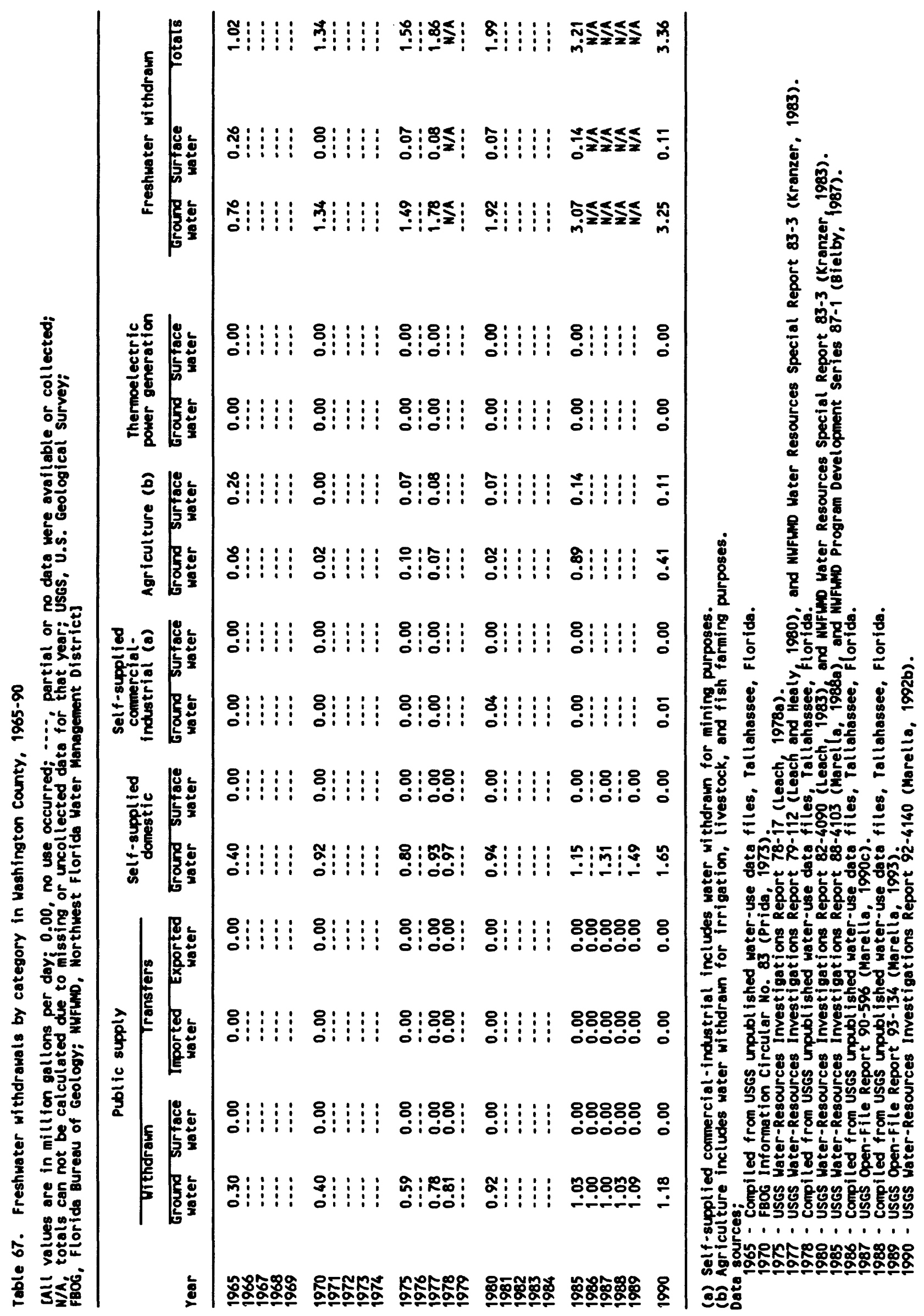

